\title{
SITE ENVIRONMENTAL REPORT FOR CALENDAR YEAR 1989
}

\author{
U.S. DEPARTMENT OF ENERGY \\ NAVAL PETROLEUM RESERVES \\ IN CALIFORNIA
}


DATE: JUN 151990

DE91 002437

REPLY TO

ATTN OF:

FE-443 (Chappelle)

SURECT: Environmental Compliance Self-Assessment

To: Director, Environmental Compliance Division, EH-232

Thru: Director, Naval Pet-oleum and oil Shale Reserves, FE-44

As directed by your memorandum of March 28, 1990, the following Environmental Compliance Self-Assessment has been prepared for submission with the Naval. Petroleum Reserves in California (NPRC) 1989 Annual site Environmental Report. As specifidd in your memorandum, this addresses environmental compliance for the period January 1990 through May 1990 as follows :

1. Comprehensive Environmental Response, compensation, and Liability Act (CERCLA) - Under the provision of this act, abandoned waste sites and unpermitted discharges which have the potential to significantly degrade the environment are identified and ranked for remedial action. The act through the Superfund Amendmerits also provides funding for the remedial action and the development of a ranking for placement on a National priority list (NPL). Sites at NPR-1 were investigated under CERCIA and ranked in 1987 . None of the sitos received scores high enough to be placed on the NPL. Even so, chromium spill sites (which received the highest ranking at NPR-1) have been remediated as of May 1.5 , 1990, and are awaiting confirmation testing to verify cleanup. This verification testing is scheduled for July 1990. Similarly, arsenic-contaminated sites are being characterized and remedial plans will be prepared. No noncompliance actions have been filed relating to CERCLA.

2. Ciean Air Act (CAA) - The Federal clean Air Act (FCAA) delegates responsibility of setting and maintaining ambient air quality standards to the EPA. The EPA in turn delegates oversight responsibility to the California Air Resources Board (CARB), which in turn delegates to the Kern County Air pollution Control District (KCAPCD). The KCAPCD has oversight responsibility for implementation of New Source Review (NSR), New Source Performance Standards (NSPS), and National Emission standards for Hazardous Air Pollutants (NESHAP) Program. These programs are all mandated by the FCAA and are enforced through the KCAPCD NSR Program. NPR-1 currently has 603 permits to operate, issued by the KCAPCD. 
The FCAA also mandated the prevention of significant Deterioration (PSD) Program which is enforced at the federal level by the EPA. NPR-1 currently has a PSD permit with the EPA covering 34 internal combustion engines.

To maintain operations, NPR-1 applies for Authorities to Construct (ATC) for each new piece of equipment or modification to existing equipment that will in turn increase or decrease emissions to the atmosphere. This procedure allows the KCAPCD to review the project to determine compliance with local, state, and federal regulations, and this assures compliance with the Federal Clean Air Act. No non-compliance actions have been filed relating to CAA at NPRC.

3. Resource Conservation and Recovery Act (RCRA) - As an oil producing operation, the waste streams and by-products associated with oil production at NPR' are exempt from RCRA provisions. However, wastes which are generated at NPRC and subject to RCRA include relatively small volumes of items such as spent solvents, paints, acids, and lubricating oil. one Interim Status Document (ISD) exists for a hazardous waste disposal site at NPR-1 which has been inactive since November 1985. This site is in the initial phase of closure, following a process developed in consultation with the state. No non-compliance actions have been filed relating to RCRA.

4. Clean Water Act (CWA) - Under the provision of this act, standards are set to prevent the discharge of pollutants into the nation's waters. Three navigable waterways which fall under the provision of the CWA exist at NPRC (Sandy creek, Broad Creek, and Buena Vista Creek), even though water is very rarely observed in the creek bottoms. No non-compliance actions have been filed relating to the CWA at NPRC.

5. Safe Drinking Water Act (SDWA) of 1974 - The SDWA provides for the establishment of a Federal underground Injection Control program which the EPA administers through Title 40 of the Code of Federal Regulations. California (being recognized as a Primacy state) has adopted the EPA guidelines for underground injection and administers its program through the provisions of Title 14 of the California Code of Regulations. NPRC operates a state-approved wastewater injection program using class II wells subject to Title 14 safeguards. No non-compliance actions have been filed relating to the SDWA at NPRC. 
Please direct any questions in this regard to Jim killen at FTS 797-6038.

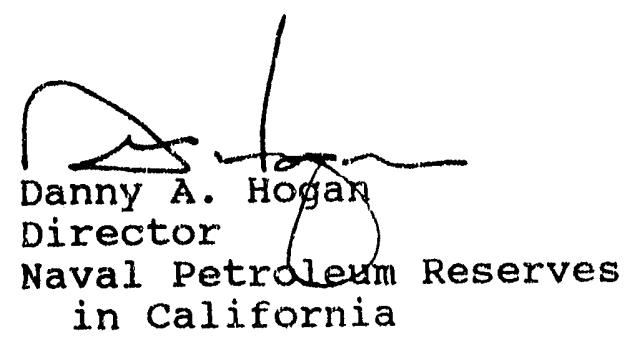




\title{
Naval Petroleum Reserves in California
}

\author{
Site Environmental Report for Calendar Year
}

1989

\section{Department of Energy}

\section{Naval Petroleum Reserves in California}

\author{
P. O. Box 11
}

\section{Tupman, California 93276}

\section{DISCLAIMER}

This report was prepared as an accuunt of work sponsored by an agency of the United States Gavernment. Neither the United States Government nor any agency thereof, nor any of their employees, makes any warranty, express or implied, or assumes any legal liability or responsibility for the accuracy, completeness, or usefulness of any information, apparatus, product, or process disclosed, or represents that its use would not infringe privately owned rights. Reference hereirı to any specific commercial product, process, or service by trade name, trademark, manufacturer, or otherwise does not necessarily constitute or imply its endorsement., recommendation, or favoring by the United States Government or any agency thereof. The views and opinions of authors expressed herein do not necessarily state or reflect those of the United States Government or any agency thereof. 


\section{TABLE OF CONTENTS}

Page

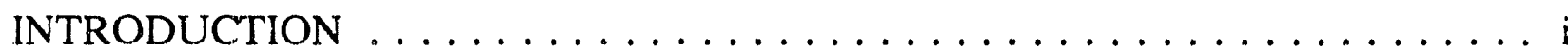

SUMMARY OF REPORT $\ldots \ldots \ldots \ldots \ldots \ldots \ldots \ldots \ldots \ldots \ldots \ldots \ldots$

COMPLIANCE SUMMARY $\ldots \ldots \ldots \ldots \ldots \ldots \ldots \ldots \ldots \ldots \ldots \ldots \ldots$

ENVIRONMENTAL PROGRAM INFORMATION $\ldots \ldots \ldots \ldots \ldots$

A. Summary of Environmental Monitoring Performed $\ldots \ldots \ldots \ldots \ldots$

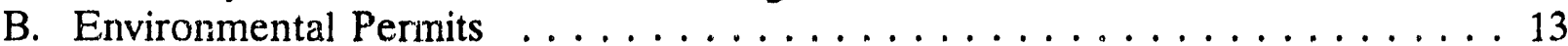

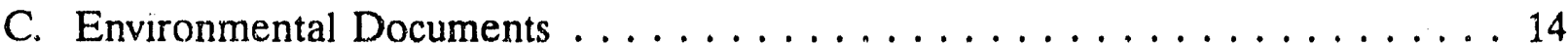

D. Summary of Significant Environmental Activities . . . . . . . . . . . 15

ENVIRONMENTAL RADIOLOGICAL PROGRAM INFORMATION $\ldots \ldots$. . . 17

ENVIRONMENTAL NON-RADIOLOGICAL PROGRAM INFORMATION . . . 18

a. Effluent Data . . . . . . . . . . . . . . . . . . . . 18

b. Environmental Sampling for Non-Radiological Pollution . . . . . . . . . . 18

GROUNDWATER PROTECTION SUMMARY $\ldots \ldots \ldots \ldots \ldots \ldots$

QUALITY ASSURANCE SUMMARY $\ldots \ldots \ldots \ldots \ldots \ldots \ldots \ldots \ldots$

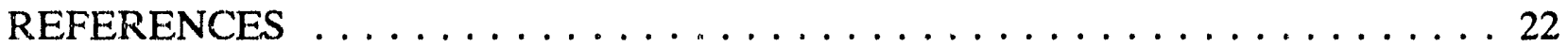

DISTRIBUTION LIST $\ldots \ldots \ldots \ldots \ldots \ldots \ldots \ldots \ldots \ldots \ldots \ldots \ldots \ldots$ 


\section{APPENDICES}

A. Topsoil Conservation Inspections List by BPOI

B. Summary of Preactivity Surveys Performed by EG\&G/EM on NPR-1 and NPR-2 for 1989

C. Summary of 1989 NPR-1 Emission Monitoring Data

D. Air Monitoring Data for 1989 from West San Joaquin Monitoring Group (BPOI member)

E. Summary of NPR-1 Oil Spills and Corrective Action Evaluation for 1989

F. Summary of 1989 Hazardous Waste Shipments from NPR-1 and 1989 Facility Annual Hazardous Waste Report to the California Department of Health Services

G. Summary of NPR-1 1989 Class II Well Data Submitted to the California Division of Oil and Gas

H. Summary of NPR-1 1989 Disposal Site Monitoring Data Submitted to the California Regional Water Quality Control Board (annual report)

I. Summary of NPR-1 1989 Samples Taken for Water, Waste, and PCBs

J. Description of Site Geology and Groundwater Aquifers

K. Compliance Issues

L. Summary of NPR-1 Environmental Permits and Other Agreements/Findings

M. Audit of Environmental Services Department Hazardous Waste Disposal Site 


\section{INTRODUCTION}

The Naval Petroleum Reserves in California (NPRC) is located about 25 miles southwest of Bakersfield, California. The NPRC consists of Naval Petroleum Reserve No. 1 (NPR1) and Naval Petroleum Reserve No. 2 (NPR-2). It is considered a large producing oil field having averaged 93,700 barrels of oil per day, 396,400 mcf/day of natural gas and 615,000 gallons/day of natural gas liquids during calenciar year 1989.

Essentially all of NPR-1 is operated according to the provisions of a Unit Plan Contract (UPC) executed in 1944 by the United States and Standard Oil Company of California (Chevron, U.S.A.). A small portion of Department of Energy (DOE) property (approximately 670 acres) on NPR-1 is not part of the UPC and is operated as $100 \%$ DOE. The production in the Stevens zone on section 31 T inside NPR-1 is owned and operated by ARCO.

Under the UPC, unit costs and production are shared in accordance with the percent of total oil reserves underlying the participant's lands (approximately 78 percent DOE and 22 percent Chevron). Operating and financial decisions under the UPC are made by an Operating Committee consisting of one government member and one Chevron member, each having an equal vote. The actual operation of the unit properties is the responsibility of the Federal Government. The Government's share of NPR-1 production is competitively bid and sold on the open market, and receipts are deposited in the U.S. Treasury's Miscellaneous Receipts Account. Chevron's share of NPR-1 production is disposed of by Chevron.

Existing operational facilities include 1,183 active wells (producers and injectors), 132 tank settings, approximately 1155 miles of pipelines and 912 miles of roadways, 5 oil cleaning/leaseautomatic custody transfer (LACT) stations, 5 wastewater disposal facilities, 3 wastewater injection facilities, 3 gas processing plants, 16 gas compression plants, 1 abandoned booster plant, 36 product storage tanks, 1 gas injection plant, and numerous pumps and compressors. Present operations include drilling, production operations, water flooding and gas injection, wastewater disposal and reuse. Most of NPR-1 is closed to the general public and grazing is not allowed.

NPR-1 has been operated by Bechtel Petroleum Operations, Inc. (BPOI), Unit Operator, since August 1, 1985 under a management and operations contract with DOE. The Unit Operator a!so performs services on NPR-2 as directed by DOE. Since 1979, EG\&G Energy Measurement, Inc. (EG\&G/EM), has been under contract to DOE for providing endangered species studies and monitoring on NPRC. 
NPR-2 covers approximately 30,080 acres and is adjacent to and south of NPR-1. The Federal Government has mineral rights ownership of about 10,400 acres (about 10,040 acres of surface rights) and the remainder is privately owned. About $90 \%$ of the Federal Government land has been leased for royalty share. There are 5 unit agreements between DOE and six lessees (ARCO, Chevron, Mobil, Phillips, Texaco and Unocal). The NPR-2 oilfield has been producing continuously since the early 1920 s and the field is near depletion. Many wells on NPR-2 are considered "stripper wells" (i.e., wells that produce fewer than 10 barrels of oil per day). DOE has no active production on its non-leased land on NPR-2. 


\section{SUMMARY OF REPORT}

Due to the differences in operation of NPR-1 and NPR-2, this summary for NPRC is divided into NPR-1 and NPR-2 discussions as follows:

\section{A. $\quad$ NPR-1}

Monitoring efforts at NPR-1 include handling and disposal of oilfield wastes; environmental preactivity surveys for the protection of endangered species and archaeological resources; inspections of topsoil stockpiling; monitoring of revegetated sites; surveillance of production facilities for hydrocarbons and oxides of nitrogen (NOx) emissions; monitoring of oil spill prevention and cleanup; and monitoring of wastewater injection.

No major compliance issues existed for NPR-1 during 1989. Activities for the NOx reduction program per the Federal Facilities Compliance Agreement with Environmental Protection Agency (EPA) were completed in 1989 as scheduled. As a result of Kern County Air Pollution Control District (KCAPCD) rule modifications, NPR-1 permitted 17\% internal combustion (IC) engines between 50500 horsepower (HP). Additionally, KCAPCD Rule 427 requires NOx emissions control on 50 IC engines at NPR-1. To date, 15 engines have been completed.

Hydrocarbon emissions are monitored to ensure compliance with KCAPCD permit conditions requiring gas-tight operation of tanks and fugitive oilfield/gas plants emissions from light oil and gas components. NOx from compressor engine exhaust streams are monitored monthly to ensure compliance with applicable KCAPCD and EPA NOx emission sampling limits.

During 1989, a comprehensive plan was developed to inventory emission of toxic air pollutants as mandated by the Air Toxics "Hotspots" Inforrnation and Assessment Act of 1987. The plan will be fully implemented in 1990 and the site emissions inventory submitted to the KCAPCD and the California Air Resources Board (CARB) on June 1, 1990. The chemical of interest at NPR-1 is benzene contained in the produced oil and gas.

The volume of used drilling fluid, tank bottoms and oily sludges disposed of in two, Class I Waste Disposal Sites is routinely monitored for monthly reporting to the California Regional Water Quality Control Board (CRWQCB). Shipments of hazardous waste (both California and EPA) off NPR-1 is reported to the State of California Department of Health Services (DHS) by sending copies of hazardous waste manifests. An effort to minimize waste at NPR-1 was begun in 1987. The 
annual report to DHS contains information on the volume reduction and estimated savings.

Most wastewater on NPR-1 is injected into wastewater disposal wells or into water flood wells to enhance oil recovery. The wastewater disposal well, water flood well data and the annual mechanical integrity survey data are reported to the California Division of Oil and Gas.

Oil spills are recorded, reviewed for corrective action, and reported to the Director, NPRC. Large spills are reported to the California Office of Emergency Services and EPA as appropriate.

Environmental preactivity surveys for proposed projects which may disturb or contaminate the land are conducted to prevent damage to the federally protected San Joaquin kit fox, blunt-nosed leopard lizard, Tipton kangaroo rat and the giant kangaroo rat. Projects are adjusted or relocated as necessary to avoid impact to dens, burrows or flat-bottomed drainages.

A major revegetation program was accomplished in 1989 for erosion control and enhancement of endangered species habitat. As part of this effort, inspections were conducted of new construction sites to ensure that topsoil had been excavated and stockpiled for future revegetation projects. In addition, third party permittees are required to perform contemporaneous reclamation and other environmental protection measures.

\section{B. $\quad$ NPR-2}

DOE NPR-2 activities include surveillance and detailed inspections of lessee activities and DOE non-leased properties by the Unit Operator as directed by DOE, and an endangered species program by EG\&G/EM as dirested by DOE.

The main compliance issue on NPR-2 was oil and produced water discharges into drainages by lessees. Under compliance ord rrs by the California Regional Water Quality Control Board, these discharges are to be eliminated by June 30, 1989. This requirement has been met by the lessees.

An additional compliance issue on NPR-2 is surface refuse from past oilfield operations. Notices of environmental deficiencies were issued to individual lessees of DOE land. Compliance with the California Division of Oil and Gas (DOG) notices should be accomplished in 1990. 


\section{COMPLIANCE SUMMARY}

The discussion of compliance issues for NPRC are divided between DOE operations on NPR-1 and DOE owned land on NPR-2. DOE also monitors ARCO's use of DOE surface rights on Section 31T on NPR-1.

\section{A. $\quad \underline{N P R-1}$}

DOE and its contractors are respousible for environmental complance on NPR-1 with the exception of the 3/4 Section of privately owned land in Section 22S; the town of Tupman in Section 24S where the surface rights are not owned by DOE or Chevron; and the portion of Section 31T inside NPR-1 where DOE owns the surface rights, but the Stevens mineral rights and the surface facilities are owned by ARCO. Following is a summary of compliance issues by category.

1. $\quad$ Air

Air quality compliance issues during 1989 included; (1) completion of the NOx Reduction Program per the Federal Facilities Compliance .Agreement with EPA; (2) one compressor in noncompliance with KCAPCD $\mathrm{PM}, \mathrm{SO}_{2}$, and $\mathrm{SO}_{4}$ emission limits; (3) gas stacking at tank settings; (4) visible emissions at the gas plant flares and elimination of such occurrences at compressor crankcase vents; and (5) other minor issues from breakdowns (Appendix K).

\section{Water}

a. California Regiona Water Quality Control Board (CRWQCB)

Waste Discharge Order 58-491 issued by the CRWQCB requires any discharge to surface drainage channels or into unlined sumps on Alluvial soils to conform to the following criteria:

1) Total Dissolved Solids content shall not exceed 1000 parts per million.

2) Chloride content shall not exceed 175 parts per million.

3) Boron content shall not exceed 2.0 parts per million.

Proposition 65, a new law passed by California voters which became applicable to certain chemicals in water in 1988 also applies to areas on NPR-1 where drinking water aquifers may be present. 
Water produced with the oil (called produced water) on NPR-1 cxceeds the linits for discharge on Alluvial soils and does contain Proposition 65 listed chemicals. Therefore, the $25 \mathrm{~S}$ tank setting located on Alluvial soils was modified to repair a leaking tank and to eliminate a smell unlined sump which received wastewater drainage. A lined secondary containment area was provided for upsets/breakdowns.

The $26 \mathrm{Z}$ wastewater facilities were modified to eliminate frequent sumping on Allaval soils and the oil production is now shut in when necessary to prevent sumping.

Upsets occurred at the $18 \mathrm{G}$ I ACT facilities which caused produced water discharges to the second ty containment areas on Alluvial soils. The problem is being addressid by providing a tank to contain upsets and lining the sump to be used for emergencies only.

Vo mes of material discharged at the $10 \mathrm{G}$ and $27 \mathrm{R}$ landfarm/disposal areas at times $r$ ray exceed the original Waste Discharge Order volume limits for the sites. New applications for revised Waste Discharge Requirements for $27 R$ and $10 G$ are scheduled to be filed in 1990 as directed by the CRWQCB. In the interirn, the volumes of materials discharged at each site are reported to the CRWQCB on a monthly basis.

Based on concerns of the potential impact on useable groundwater aquifers, NPR-1 is proceeding with elimination of all surface wastewater sumps by 1991 . During 1989 , seven (7) sumps at $26 \mathrm{Z}$ and four (4) sumps at $14 Z$ were closed, regraded and revegetated.

An estimated 3000 barrels per day of produced wastewater in FY89 will be injected into the Olig formation instead of the Tulare formation in 26Z. By the end of FY91, an additional 54,000 barrels per day will be removed from Tulare injection and reused in the Stevens waterflood in place of Tulare water. An additional volume of about 20,000 barrels per day of Shallow Oil Zone (SOZ) wastewater injected into the Tulare zone and future increases in production of wastewater are planned for reuse or alternate disposal by the end of FY94. 
b. California Division of Oil and Gas (DOG)

DOG regulates all California oilfield activities and is the EPAapproved agency for California for Class II wells under the Safe Drinking Water Act. Several notices of deficiency were sent to NPR1 during 1989. Main concerns identified by the DOG were well mechanical problems (packer leaks) and improper abandonment procedures (Appendix K).

3. Waste

NPR-1 does not have any current waste disposal compliance issues. A question of labeling pract' es by the DOE Environmental Survey Team for out-of-service PCB transformers awaiting proper disposal was resolv $d$ by a change in labeling procedures. Proper closure of ine out-of-service (isposal trenches at $27 R$ and possible CERCLA sites (non-National Priority List) are proceeding with the California Department of Health Services (DHS) and the CRWQCB. Remedial action levels at the chromium spill sites (nonNational Priority List) have been agreed to by the DHS and CRWQCB. Work is in progress. Closure of the out-of-service solid waste disposal sites at $35 R, 25 S$ and $26 S$ is being done in conjunction with the CRWQCB, Kern County Health Department and the California Solid Waste Management Board. Remedial action on some underground storage tank leak sites has been completed in conjunction with the Kern County Health Department and the CRWQCB.

4. National Environmental Policy Act (NEPA)

Work on a Supplemental Environmental Impact Statement for NPR-1 continued in 1989. A draft report will be issued in 1990 and a final report issued in 1991.

NEPA reviews were conducted for third party projects involving NPR-1 lands in 1989. Categorical Exclusions were done for projects which were of no significant impact.

5. Historical Preservation/Cultural Resources

Preactivity surveys were requested for $\$ 44$ projects. Most findings were documented by reports from an archaeologist under contract with EG\&G/EM. A Memorandum of Understanding with the California State Historic Preservation Officer has not yet been developed as required by the 
National Historical Preservation Act. This activity is scheduled for completion in 1990 .

\section{Erosion Control}

Use of sound erosion control measures is a commitment in the 1979

NPR-1 Environmental Impact Statement. Erosion control is also a specific vommitment of the pilot steamflood Environmental Assessment (1985). The NPRC Long Range Plan includes an erosion repair program to begin in FY90.

A field scale contemporaneous revegetarion program was initiated in 1989. At years ind, 14 sites totaling 40 acres were completed. The purpose of contemporaneous revegetation is to minimize the extent of disturbed areas as well as to optimize erosion control.

7. Wildlife

A single golden eagle was reported accidentally electrocuted. The pole was retro-fitted with a raptor protection stand to prevent the occurrence of similar incidents. The incident was included in the 1989 Annual Report per U.S. Fish and Wildlife Service permit No. 725407.

Under requirements of the U. S. Fish and Wildlife Service Biological Opinions (1985 and 1987) for NPRC, 862 environmental preactivity endangered species surveys were conducted in 1989 . No non-compliances were found (Appendix B).

\section{B. $\quad$ NPR-2}

DOE non-leased lands are DOE's compliance responsibility. No compliance issues were identified on DOE non-leased lands.

Lessees are responsible for operating their leases in compliance with laws and regulations. DOE does not have specific regulations for lessees, such as Department of Interior (DOI) has for the Bureau of Land Management (BLM) lessees and the DOI regulations do not apply to DOEs lessees. DOE does provide surveillance under the authority of the lease agreements, laws and other applicable regulations. 
With one exception, compliance issues noted and reported by DOE for lessees were minor, such as sump netting, well cellar maintenance and oil spill cleanup. The discharge of oil and produced water into "navigable waterways" is a more significant compliance issue and enforcement action by the CRWQCB existed. Lessees are under orders from the CRWQCB to cease these discharges into Sandy, Broad or Buena Vista Creek or their tributaries by June 30, 1989. The lessees met the requirement in 1989.

In 1989 , the DL 3 conducted inspections of NPR-2 with primary attention on surface refuse resulting from past oilfield operations. The lessees have been issued notices of environmental deficiencies regarding surface clean-up to bring about compliance with DOG regulations. Compliance shculd be met by the lessees in 1990.

A NEPA document is planned for NPR-2 in 1990-1991. Separate NEPA reviews were completed in 1988 for the installation of City of Taft monument type entrance signs and a Mobil cathodic protection well on DOE, NPR-2 leased lands. The NEPA review for several other third party projects which crossed DOE land on NPR-2 were included in the NPR-1 NEPA review since they were also on NPR1. A Negative Declaration was filed by the West Kern Water District in late 1988 for expansion of their freshwater supply system.

Environmental preactivity surveys are also performed on DOE land on NPR-2 lessee projects and third party projects. Records are kept of the preactivity surveys performed by EG\&G/EM (Appendix B). If the preactivity survey is not performed by EG\&G/EM, the person conducting the preactivity survey must be acceptable to DOE. DOE is keeping records of preactivity surveys done directly by lessees and third parties in 1989. 


\section{ENVIRONMENTAL PROGRAM INFORMATION}

\section{A. SUMMARY OF ENVIRONMENTAL MONITORING PERFORMED}

Environmental monitoring on NPR-1 is different than on NPR-2, except for NPR-1 Section 31T where only ARCOs use of DOE surface rights is monitored. All petroleum operations on NPR-1 are monitored, whereas NPR-2 monitoring involves only the petroleum activities of DOE lessees and DOE unleased lands. Operations of private oil companies on non-DOE NPR-2 lands are not monitored. The detailed environmental monitoring information presented below is separated into NPR-1 and NIR-2 sections.

\section{NPR-1}

DOE monitoring efforts at NYPR-1 include air, water, waste and wildlife as described below. Monitoring is performed by the Unit Operator and its subcontractors and EG\&G/EM.

\section{a. $\quad$ Air}

Air monitoring is performed to assure compliance with air regulations and includes participation in a local petroleum industry air monitoring group to establish meteorological and air quality data.

1) Oxides of Nitrogen Testing Monthly tests are performed on compressor engines for oxides of nitrogen emissions. Five to eight engines are tested per month. The testing was previously required under a Prevention of Significant Deterioration Permit (NSR4-4-8/SJ77-42) issued by EPA, Region IX (Appendix C-I).

An extensive NOx emissions reduction program was completed in 1989 to ensure compliance with the Federal Facility Compliance Agreement with EPA, Region IX. Major reductions in oxides of nitrogen and carbon monoxide were made. Compliance testing for the modified compressors was performed in 1988 and 1989 with KCAPCD present to witness the testing. The modification program was successfully completed in February 1989 in accordance with the established schedule milestones. 
2) Tank Inspections Monitoring is performed on fugitive hydrocarbon emissions from oilfield tanks on NPR-1. Monitoring is done to ensure compliance with KCAFCD Regulation 411 and Permit to Operate (PTO) requirements, but it is not required to be reported to KCAPCD. Tanks having total hydrocarbon emissions exceeding $10,000 \mathrm{ppm}$ non-methane and ethane hydrocarbons are repaired to ensure compliance (Appendix C-IV).

3) KCAPCD Compliance Monitoring As part of the NOx emission reduction program, compressors modified to reduce NOx have specific emission limits set for each compressor. After modification, each compressor is tested to verify compliance with the applicable permit limits and annually thereafter (Appendix C-II).

4) KCAPCD Rule 414.7 Fugitive Emissions monitoring This rule requires valves, flanges and other oilfield components in light oil production and gas plants to be monitored for fugitive hydrocarbon emissions. The rule requires repair of the leaks. This is a major labor intensive program at NPR-1. Two men full tine are required for the program. Over 500,000 components were inspected with a leak rate less than $1 \%$ found (a leak is defined as an emission of $20,000 \mathrm{ppm}$ volatile organic compounds at 1 centimeter from the source) in the first year of the program (Appendix C-III).

5) Air Quality Monitoring In 1989, NPRC joined the West San Joaquin NOx monitoring group, an industry group with meteorological and air quality monitoring stations near NPRC. Data from the monitoring network show violations of California and Federal ambient standards for ozone and Particulate Matter 10 (PM-10) in Western Kern County. Other Federal criteria pollutants are in attainment. NOx and hydrocarbons are precursors of ozone. NOx is produced from combustion sources. Hydrocarbons emissions are from production operations and natural sources. PM-10 is from natural sources, roads both paved and unpaved, combustion sources and secondary aerosols from NOx, hydrocarbons and SOx emissions. Data from the NOx monitoring group stations is also being used for air emissions modeling being done in conjunction with preparation of the NPR-1 Environmental Impact Statementi, and 
for modelling required to obtain air emissions permits (Appendix D).

b. Water

Water monitoring on NPR-1 consists of monitoring to the requirements of the CRWQCB Discharge Orders for the two lanc treatment/disposal sites (Appendix $H$ ); occasional tests of various waters and evaluation of NPR-1 discharges in relationship to California Proposition 65 (Appendix I); monitoring of injection well pressures and volume of liquid injected, both waterflood and wastewater disposal for the DOG; and mechanical integrity testing of both waterflood and wastewater disposal wells per DOG requirements (Appendix $G$ ).

c. Waste

Waste monitoring on NPR-1 consists of keeping track of Federal and/or California hazardous wastes sent off NPR-1, including an annual report to the DHS (Appendix F); sampling for PCBs in transformers (Appendix I); sampling of soils and other wastes to determine if they are California or Federal hazardous wastes (Appendix I); and tracking NPR-1 oil spills, including corrective action (Appendix E). Additional monitoring includes daily surveillance of NPR-1 for compliance issues and other environmental problems, and a special survey each year of well pads to visually determine if evidence of past hexavalent chromium spills have been brought to the surface by evaporation after the annual rains. One new hexavalent chromium spill site was found in 1989, and several new stain areas showed up on a well pads that already had been cleaned up. Hexavalent chromium was used as a drilling mud additive during the period 1955 through 1983 at up to approximately 550 well pads on NPR-1. To date, 62 spill sites have been located and all but about 20 have been cleaned up. Final cleanup of known sites is underway and is to be completed in FY90.

d. Wildlife

Most monitoring for wildlife is concentrated on endangered species, but revegetation monitoring efforts beneficial to all wildlife on NPR-1 is also done.

Endangered species protection monitoring focuses on four animal species currently listed as endangered under the Endangered Species 
Act and one plant species currently being proposed for listing as threatened known to occur on and adjacent to NPRC lands. These are the San Joaquin kit fox (Vulpes macrotis inutica), giant kangaroo rat (Dipodomys ingens), Tipton's kangaroo rat (Dipodmys nitratoides), blunt-nosed leopard lizard (Gambelia silus), and Hoover's woolly-star (Eriastrum hooveri). Pursuant to protection guidelines issued by the U.S. Fish and Wildlife Service, in their Biological Opinions of 1980 and 1987, proposed construction sites (e.g., well locations, pipeline routes, roadways, building sites) and land disturbing activities are examined for evidence of impact on the species or their habitat immediately prior to any activities. Proposed actions that would, for instance destroy a kit fox den site, are modified or relocated to avoid impact (Appendix B).

The NPR-1 kit fox population is monitored twice a year to gather information on distribution and abundance of this species. Populations of predators of the foxes (primarily coyotes) and the principle prey of foxes (lagamorphs) are monitored three times a year. This population monitoring condunted by $E G \& G / E M$ is consistent with the recommendations made by the U.S. Fish and Wildlife Service in their Biological Opinions of 1980 and 1987 regarding petroleum production at maximum efficient rate on NPR-1.

Conservation of topsoil for future revegetation efforts is also an important program on NPR-1. Activities which disturb soils require the removal of the top 12 inches of soil. These activities are closely monitored and an inventory of stockpiled soils is maintained (Appendix A). The habitat reclamation monitoring program conducted by EG\&G/EM since 1987 evaluates revegetation success on NPR-1. In 1989, 553 sites revegetated in the fall of 1987 and 1988 were evaluated (EG\&G Energy Measurements, Inc. 1990).

e. Archaeology

The preactivity surveys for endangered species include inspection for the presence of archaeological resource (Appendix B). One site found in 1989 was considered to be of significant archaeological value and was reviewed by an archaeologist under contract. This site in $36 \mathrm{~S}$ was found to contain artifacts from oilfield activities in the early 1900 's. The site was not disturbed by the project. 


\section{2. $\quad$ NPR-2}

DOE monitoring on NPR-2 consists of general environmental monitoring by the Unit Operator, and endangered species monitoring by EG\&G/EM.

\section{a. General Environmental Monitoring}

Monitoring of NPR-2 by the Unit Operator consists of twice monthly surveys of DOE property, and an ongoing detailed environmental survey of DOE lands which were completed in 1989.

The Unit Operator also monitors spills and other special activities of lessees on an individual basis to assure proper cleanup and reporting. Special activities consist of such items as well drilling, well abandonment, general surface clean-up, and revegetation. Lessees are required to file a Sundry Notice to obtain DOE approval before any changes are made to DOE lands.

Monitoring of NPR-2 for 1989 has not shown any major problems and has shown improvement in environmental conditions. Tl.e Environmental Plan for NPR-2 dated September 1986 was drafted for DOE by the Unit Operator. The Plan is presently being updated to include present conditions before final issuance.

Detailed NPR-2 environmental surveys consist of comprehensive walkthrough inspections of DOE land, both leased and un-leased, and the issuance of reports identifying the property condition with emphasis on environmental concerns. In 1989, detailed environmental surveys were completed on all of the approximately 10,040 total NPR-2 acres owned by DOE (DOE surface rights).

\section{b. Endangered Species Monitoring}

Preactivity surveys for federally protected endangered species were conducted by EG\&G/EM for DOE projects and for third-party projects that took place on or crossed NPR-2 property owned by DOE. Four endangered species are found on NPR-2: the San Joaquin kit fox (Vulpes macrotis mutica), giant kangaroo rat (Dipodomys ingens), Tipton's kangaroo rat (Dipodomys nitratoides), and the blunt-nosed leopard lizard (Gambelia silus) Proposed construction sites (i.e., well locations, pipeline routes, roadways) and lands to be disturbed by activities such as geophysical surveys were examined for the presence of endangered species and possible impacts 
to the species. When appropriate, proposed activities were nodified to avoid impacts to endangered species.

The NPR-2 kit fox population is monitored twice a year to gather information on distribution and abundance of this species. Populations of predators of foxes (primarily coyote) and the principle prey of foxes (lagamorphs) are monitored three times a year. This population monitoring conducted by EG\&G/EM is consistent with the recommendations made by the U.S. Fish and Wildlife Service in their Biological Opinions of 1980 and 1987 regarding petroleum production at the maximum efficient rate on NPR-1.

\section{B. ENVIRONMENTAL PERMITS}

Following is a list of environmental permits by agency:

1. $\quad \underline{E A}$

1. Prevention of Significant Deterioration Authority to Construct A-3-INSR4-4-8/S5;77-42

2. EPA identification number CA4170024414

2. U. S. Fish and Wildlife Service

See Appendix L

3. California Dept. of Fish \& Game

A Memorandum of Understanding was signed by the Department of the Navy for NPRC with the California Fish and Game Department in 1963. The Memorandum of Understanding does not have an expiration date.

\section{California Regional Water Quality Control Board}

1. 58-491 Waste Discharge Requirements for wastewater discharge. No expiration date.

2. 73-42 Waste Discharge Requirements for Class II-1 disposal area at $10 \mathrm{G}$. No expiration date.

3. 73-141 Waste Discharge Requirements for Class II-1 disposal area at $27 R$. No expiration date. 


\section{California Solid Waste Board}

These permits are administered by the Kern County Health Department (KCHD) and consist of the Solid Waste Information System (SWIS) permits listed under KCHD (Appendix L-IV).

\section{California Department of Health Services}

Interim Status Document for $27 \mathrm{R}$ disposal trenches Number CA4170024414. NPRC has requested cancellation since the site is no longer used.

7. California Division of Oil and Gas (DOG)

DOG permits are issued for each well activity and are no longer used when the activity is completed. DOG's files track wells by operator, well number, township and range (Appendix $G$ ).

\section{Kern County Air Pollution Control District}

The PTO expire annually (Appendix L-1). A total of 354 PTOs existed for NPR-1 as of 12/31/89. Also included in Appendix L are current authorities to construct which expire when PTO's are issued.

\section{Kern County Health Department}

Various facilities on NPR-1 require permits for the KCHD (Appendix L). KCHD also issues permits for construction of cathodic protection wells, but the permits expire when the well is completed and the permit numbers are for their records only. According to their records, 58 permits were issued for NPR-1 in 1989.

\section{ENVIRONMENTAL DOCUMENTS}

NEPA reviews were conducted for third farty projects in 1989. None were found to require an EIS or EA because preparation of a supplemental EIS for NPR-1 is in progress. A NEPA review is also planned for NPR-2. The Negative Declaration by the West Kern Water District for an expansion of their freshwater system which includes DOE land on NPR-2 is under DOE review. 


\section{SUMMARY OF SIGNIFICANT ENVIRONMENTAL ACTIVITIES IN 1989}

\section{Solid Waste Sites}

Work on closure of the three solid waste sites taken out of service permanently in 1987 continued in 1989 . The sites were cleaned up and contoured. Final closure is awaiting the results of a site characterization to be completed in 1989. After site characterization, closure plans will be developed with the CRWQCB, State Waste Management Board and the Kern County Health Department.

2. Comprehensive Environmental Response, Compensation and Liability Act (CERCLA) Potential Sites

Work continued in 1989 on potential CERCLA sites with further investigations of several small sites to address CRWQCB concerns. Further site investigation work for the chromium spill sites and arsenic sites was approved.

A site evaluation report was completed on the $4 \mathrm{G}$ W-41 disposal site with the finding by BPOI that the site only contains items such as valves, tubing and pipes which were used for W-41, some of which still contain arsenic. W-41 was an arsenic containing corrosion inhibitor used on NPR-1 in inactive SOZ wells for corrosion protection. Remedial action planned for 1989 will remove the remaining arsenic containing equipment so closure as a waste disposal site will not be necessary.

\section{27R Disposal Site}

The replacement of the existing open 27R oil recovery and truck wash out sumps with a tank was authorized in early 1989. Project completion is planned for late 1990 . The project was delayed due to changes in the air quality rules in mid 1989 by Kern County.

Several meetings were held with representatives of the California DHS and the CRWQCB Board to review closure of the 27R disposal trenches. The disposal trenches have been investigated and found to not contain California hazardous waste levels of contaminates. A risk analysis and site background investigation was also performed on the disposal trenches and they were found to not present a significant hazard. The agencies have determined that the site will be "closed" 
as a regulated unit. Work will continue in 1990 to determine the specific closure and post closure requirements.

Further, work was completed on an investigation of the possibility of a perched aquifer below the truck washout area to address concerns of the DHS and CRWQCB. This investigation showed no evidence of such an aquifer.

In addition, work is in progress to update the waste discharge requirements for the $27 \mathrm{R}$ and $10 \mathrm{G}$ landfarm/disposal areas.

\section{Underground Tanks}

Work was completed to investigate and characterize the extent of any contamination d'se to leaks from six underground tanks on NPR-1. The tanks were excavated, removed from NPR-1, and replaced where needed with above ground tanks in 1987 . The report was submitted to the Kern County Health Department which issued a finding that no further investigation or remediation was necessary at the site.

\section{NOx Emission Reductions}

The NOx emissions reduction program at NPR-1 was completed in 1989 resulting in reductions of over $12,000 \mathrm{lbs} /$ day NOx emissions. The reductions (over 10,000 lbs/day formally approved EPA NOx reduction credits) exceeded the reductions required by the Federal Facility Compliance Agreement. Permitted NOx emissions from 15 of the modified engines are 2.00 grams per brake horsepower-hour versus $7.00 \mathrm{gm} / \mathrm{bhp}^{*} \mathrm{hr}$ required by the Compliance Agreement (Appendix K-II). 
NPRC is not a nuclear facility and monitoring is not required under Federal, State and local regulations. Preparation for an initial survey of naturally occurring radioactive materials was accomplished in 1988, and the survey of selected NPR-1 facilities was completed in January 1989. Initial results show exposures well below DOE administrative levels. 


\section{ENVIRONMENTAL NON-RADIOLOGICAL PROGRAM INFORMATION}

\section{A. EFFLUENT DATA}

1. $\underline{\text { Air }}$

$\underline{\text { NPR-1 }}$

See Appendix $\mathrm{C}$ for data on emissions monitoring for compressors on NPR1 , fugitive emissions test program for production and gas plant valves and flanges, and fugitive emission testing program for tanks. NPR-2 lessees are responsible directly to the KCAPCD for air emissions, permits, and related compliance requirements.

2. Water

NPR-1 discharges water to sumps and Class II injection wells (Appendix GIII). Water analyses are not required on a regular basis for NPR-1 discharges. Records of discharges to the $27 \mathrm{R}$ and $10 \mathrm{G}$ land farm/disposal areas are reported in Appendix $\mathrm{H}$. Work is in progress to update the Waste Discharge Orders for these sites, so volumes disposed do not exceed original volumes approved.

Some NPR-2 lessees did discharge to ephemeral streams on NPR-2 prior to 1989. Volumes discharged and concentrations of constituents were the responsibility of the lessee to report to the CRWQCB. All NPR-2 lessee discharges to ephemeral streams on NPR-2 ceased in early 1989.

\section{B. ENVIRONMENTAI. SAMPLING FOR NON-RADIOLOGICAL POLLUTION}

1. Air

NPR-1 has joined the West San Joaquin Monitoring Group which has monitoring stations near NPR-1 (Appendix D). The monitoring network recorded exceedances of the Federal ambient ozone standard at the town of Maricopa near NPR-1. These exceedances caused implementation in 1989 of an additional oxides of nitrogen control program for the west side of Kern County including NPRC.

Exceedances of the federal PM-10 standard were also recorded by the monitoring network. A non-attainment plan is being developed by the 
KCAPCD and the CARB to address causes of PM-10 exceedances. NPRC emits minor amounts of PM-10 directly from facilities such as combustion sources. Dirt roads and re-entrained dust from paved roads on NPRC contribute to PM-10 levels. Secondary aerosols from $\mathrm{SO}_{2}$ and NOx emissions also contribute to PM-10 levels. NPRC emits only minor amounts of sulfur dioxide and major NOx emission reductions have been made as discussed in Appendix K.

Federal attainment pollutants in the NPRC area include $\mathrm{NO}_{2}, \mathrm{SO}_{2}$ and $\mathrm{CO}$.

The California ambient air quality standards for PM-10 and ozone were also exceeded in the West side of Kern County in 1988. The California standards for other pollutants were met at the West San Joaquin Monitoring Group sites.

2. Water

NPR-1

Tulare zone water (source water for the water floods)

Frequent analyses are performed of the Tulare zone source water wells on NPRC. These analyses are performed by the on-site lab and are not for regulatory purposes. Data from these analyses show these wells supply water having over $4000 \mathrm{ppm}$ total dissolved solids which exceeds potable water standards. This water is used in the waterfloods of reservoirs to increase oii production.

\section{Sumps}

NPR-1 has an active sump elimination program with sumps eliminated in Sections $26 \mathrm{Z}$ and $1.4 \mathrm{Z}$ in 1989 . The $10 \mathrm{G}$ sumps, $35 \mathrm{R}$ sump, $18 \mathrm{G}$ sumps, the 27R oil recovery and truck wash out sumps are planned for elimination in 1990.

3. Wastes and soils

Testing by California certified laboratories was done on various samples of wastes spills, and discharges sites (Appendix I). Results were compared against California hazardous waste standards. Results were below hazardous waste levels for all samples except known CERCLA sites (4G disposal site, $1 \mathrm{~A}-6 \mathrm{M}$ site, $23 \mathrm{~S}$ sumps and chrornium spill sites). 


\section{GROUNDWATER PROTECTION SUMMARY}

Activities and geology of NPRC vary between NPR-1 and NPR-2. The following discussion is divided accordingly (See Appendix $J$ for a detailed discussion of NPRC hydrogeology.)

A. NPR-1

Oilfield activities are exempt from the Resource Conservation and Recovery Act (RCRA), so RCRA groundwater monitoring is not performed. Studies to date of NPR-1 disposal sites and CERCLA sites do not show groundwater contamination. These studies were required by the State of California and by DOE Orders.

NPR-1 does have an extensive produced water injection program under the auspices of the California Division of Oil and Gas (DOG). NPR-1 is working with the CRWQCB and DOG on the NPR-1 injection well issue. The Tulare zone where most produced water is injected is a federal Safe Drinking Water Act Class II exempted aquifer in the Elk Hills Oil Field. In 1988, DOG issued specific requirements prohibiting injection of hazardous waste into Tulare injection wells.

Geologic strata below the Tulare zone contains saline water above $10,000 \mathrm{mg} / 1$ Total Dissolved Solids and do not require protection as a drinking water aquifer under the federal Safe Drinking Water Act.

Drinking water aquifers are present on the north, east and possibly the south sides of NPR-1. In order to ensure protection of these aquifers, NPR-1 is eliminating all oilfield sumps.

B. NPR-2

DOE does not presently have activities on NPR-2 affecting groundwater. DOE lessees have sumps and do inject into the Tulare zone on N:PR-2. Discharges into drainages by lessees could also affect groundwater, but these discharges were eliminated in early 1989 . The CRWQCB presently allows sumps and Tulare injection on NPR-2 by the lessees. Studies of these issues were done by lessees at the direction of the CRWQCB and delivered to the CRWOCB in January 1988. To DOE's knowledge, no actions by the CRWQCB have been taken based on the study findings. Most NPR-2 lessees have indicated that they plan to eliminate the usage of sumps for disposal of produced water. Several sumps were taken out of operation in 1989 and are planned for proper closure contingent on DOE approval. 


\section{QUALTTY ASSURANCE SUMMARY}

Samples are collected either by BPOI Environmental Services Departrient staff or subcontractors and submitted for specific analyses to State of California certified laboratories. The Environmental Services Department has a laboratory inspection program and subcontractors quality plans and programs are reviewed for quality assurance. NPR2 lessees are responsible for their own quality program. Data presented by NPR-2 lessees to DOE is reviewed for data.

Inspections are also conducted by Environmental Services Department staff of the offsite hazardous waste disposal sites. In 1989, the facilities of Environmental Services of Idaho (ESI) were inspected and the results are included as Appendix M. 


\section{REFERENCES}

1. U.S. Department of the Interior, February 1, 1980 letter of 1 February from R. Kahler Martinson, Regional Director, U.S. Fish and Wildlife Service (USFWS), to Captain Gordon R. Gilmore, Office in Charge of Construction, Naval Facilities Enginecring Command, San Bruno, California

2. USFWS. 1987 Biological Opinion for Maximum Efficient Rate

3. USFWS. 1985 Biological Opinion for the SOZ Steam Flood Project

4. Environmental Protection Agency (EPA) Prevention of Significant Deterioration Permit NSR 4-4-8/SJ 77-42

5. K.CAPCD (KCAPCD) Rules and Regulations

6. California Regional Water Quality Control Board (CRWQCB), CCR Title 23

7. California Department of Health Services (DHS), CCR Title 22

8. California Division of Oil and Gas (DOG) CCR Title 14

9. Solid Waste Management Board CCR Title 14, Div. 7

10. California Health and Safety Code

11. Cahifornia Water Code

12. California Natural Resources Code

13. California Government Code

14. California Fish and Game CCR Title 14

15. EG\&G Energy Measurements, 1990. Endangered Species Program, Naval Petroleum Reserves in California, Annual Report FY89. U.S. Department of Energy Topical Report, EG\&G/EM Santa Barbara Operations (in preparation).

16. Endangered Species Act including latest revision

17. Comprehensive Environmental Response, Compensation and Liability Act (CERCLA) including latest revisions 


\section{DISTRIBUTION LIST}

This report is distributed by Director, Naval Petroleum Reserves in California to:

\section{CONGRESSMEN}

Senator Alan Cranston

Senator Pete Wilson

Representative Thomas

\section{LOCAL NEWS MEDIA}

Bakersfield Californian (Upon Request)

Daily Midway Driller (Upon Request)

\section{APPROPRIATE STATE AND LOCAL OFFICIALS}

Governor George Deukmejian

Senator Don Rogers

Assemblyman Phillip Wyman

Assemblyman Trice Harvey

Keiii Cüniy Buard of Supervisors

\section{STATE ENVIRONMENTAL OFFICES}

Oil and Gas Division

Regional Water Quality Control Board, Fresno Office

California Air Resource Board

California, Department of Health Services

California, Health and Welfare Agency

California Water Resource Division

California Dept. of Fish and Game

Kern County Water Agency

Kern County Air Pollution Control District

Kern County Health Department

\section{FEDERAL AGENCIES}

U.S. Environmental Protection Agency, Region IX

U.S. Fish and Wildlife Service

DOE

Office of Environmental Guidance and Compliance (EH-23) 5 copies

Office of Environmental Audit (EH-24) 2 copies

Office of NEPA Project Assistance (EH-25) 2 copies

Director of Naval Petroleum and Oil Shale Reserves (DNPOSR)

NPR-1

Department of Energy - 5 copies

Chevron USA, CMOC - 5 copies

Bechtel Petroleum Operations, Inc. 2 copies

EG\&G/EM - 2 copies 


\author{
APPENDIX A
}

\title{
LIST OF TOPSOIL CONSERVATION \\ INSPECTIONS CONDUCTED BY BPOI
}


TOPSOIL INSPECTION REPORTS FOR 1989

\author{
$352 X-3 G$ \\ $33 X-10 G$ \\ 119-11G \\ $21 \mathrm{NW}-16 \mathrm{G}$ \\ 323-33R \\ $42-36 R$ \\ $316-36 \mathrm{R}$ \\ 28NE-28S \\ $327-34 \mathrm{~S}$ \\ $338-34 \mathrm{~S}$ \\ $367-345$
}

06-15-89

01-16-89

09-18-89

01-31-89

05-09-89

03-01-89

03-01-89

01-23-89

04-26-89

10-23-89

05-02-89 


\section{APPENDIX B}

SUMMARY OF PREACTIVITYY SURVEYS PERFORMED BY

EG\&G/EM ON NPR-1 AND NPR-2 FOR 1989 


\section{$\underline{\text { NPR-1 }}$}

In CY89, 862 preactivity surveys were conducted for Unit Operator and third party projects to prevent or reduce direct impacts of oil production activities on the four endangered animal species and one proposed plant species known to occur on NPR-1 (Table 1). The preactivity surveys also included surveying for the presence of cultural artifacts on each site (Table 1).

Of the 1563 acres surveyed, 638 acres (41\%) were scheduled to disturb previously undisturbed habitat (Table 1). During the surveys 124 kit fox dens, 4 blunt-nosed leopard lizards, 29 washes representing blunt-nosed leopard lizard habitat, and 61 giant kangaroo rat burrows were observed (Table 2).

Recommendations for project alterations were made on $172(20 \%)$ of the projects to minimize impact to the endangered species and their habitat. The majority of these changes $(51 \%)$ were associated with pipeline projects and clean-un of oil spills. Seven potential kit fox dens were excavated prior to construction (Table 1).

\section{NPR-2}

In CY89, 38 preactivity surveys were conducted on NPR-2 (Table 3). Of the 194 acres surveyed, 1 acre $(0.5 \%)$ was scheduled to disturb previously undisturbed habitat. A total of 13 kit fox dens, 1 blunt-nosed leopard lizard, and 1 wash representing bluntnosed leopard lizard habitat were observed during the surveys (Table 4).

Recommendations for project alterations were made on $9(53 \%)$ of the projects to minimize impact to endangered species and their habitat (Table 3). 


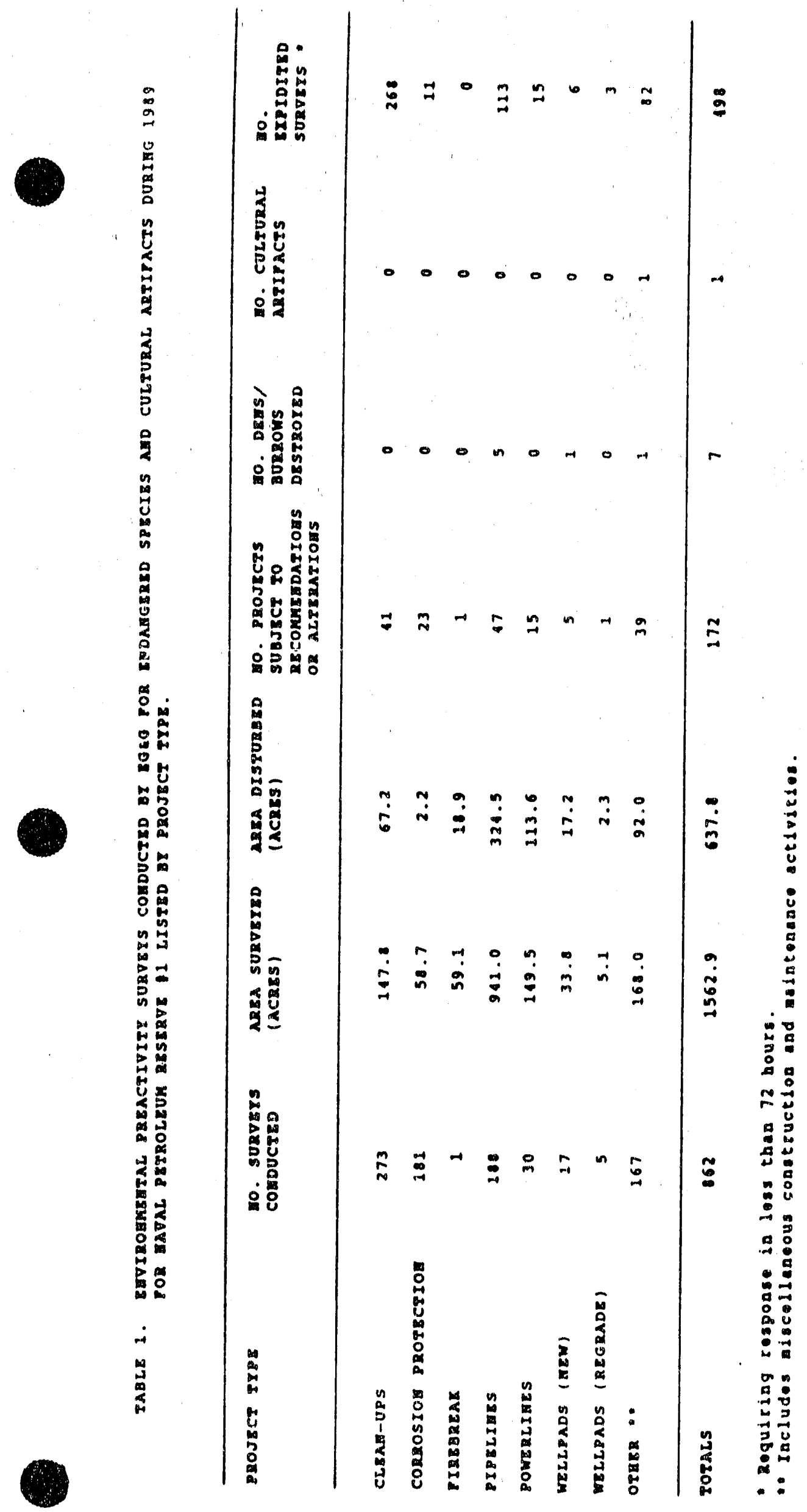




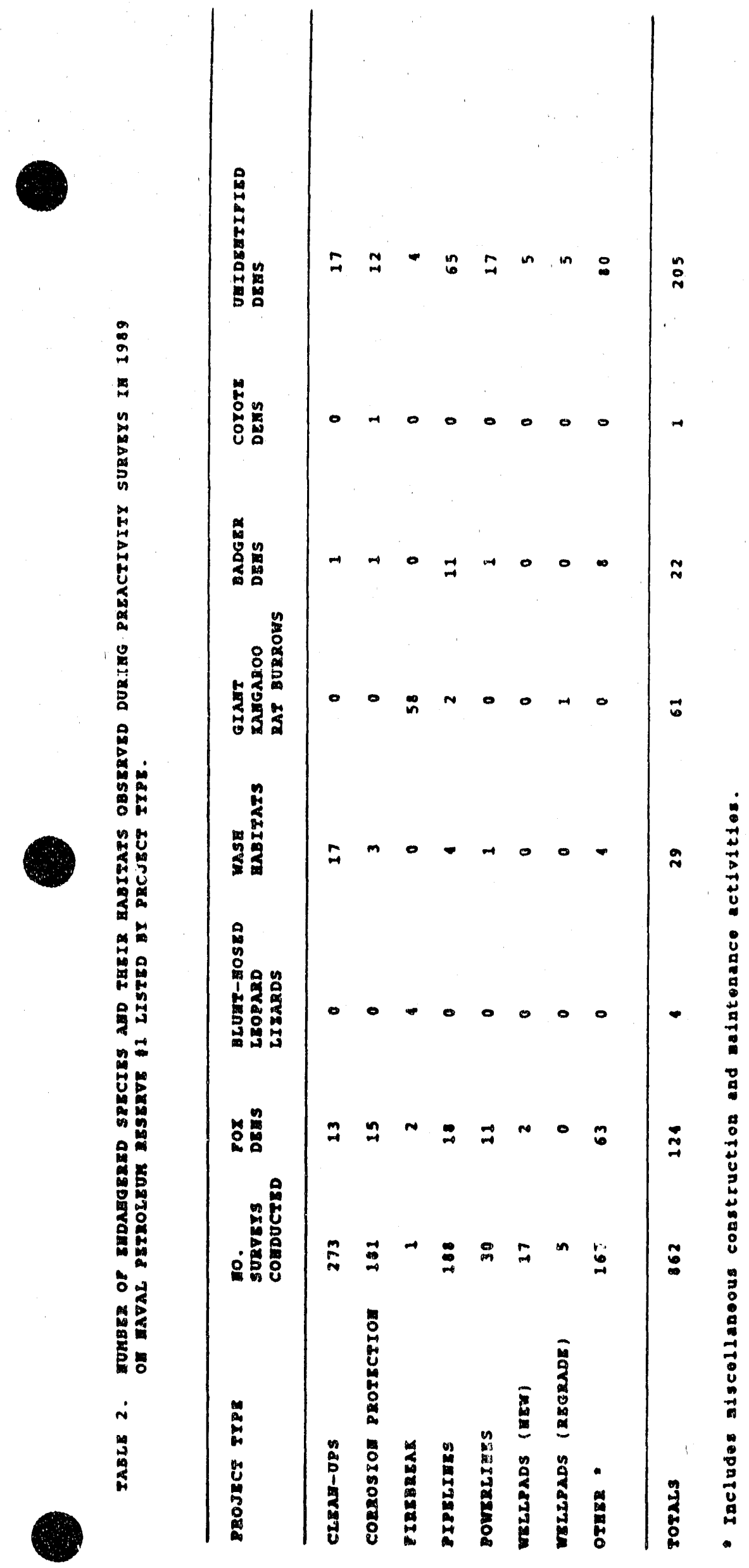




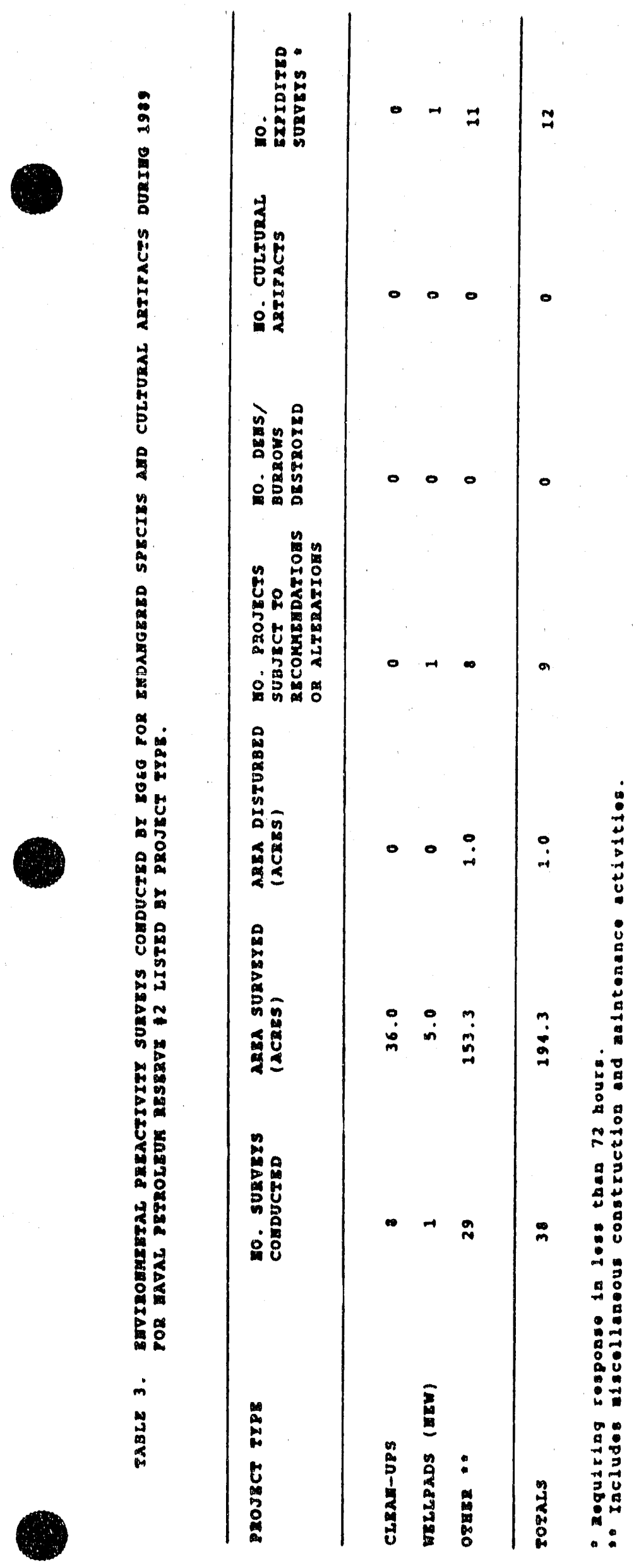




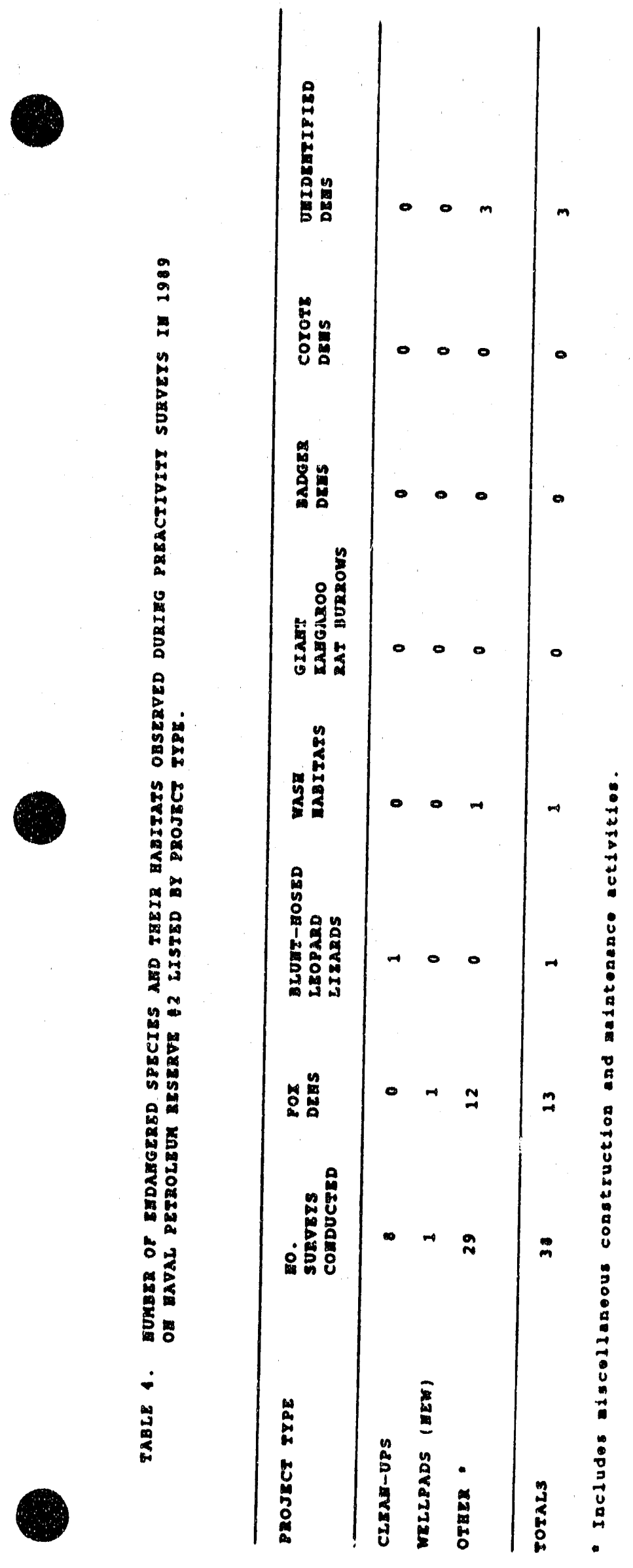




\section{APPENDIX C}

SUMMARY OF 1989 NPR-1 EMISSION MONITORING DATA 


\section{TABLE OF CONTENTS APPENDIX C}

I. Summany of 1989 NPR-1 I.C. Engine NOx Test Schedule

II. Summary of 1989 NPR-1 I.C. Engine Source Test Data for EPA

III. Summary of 1989 NPR-1 KCAPCD Rule 414.7 Fugitive Emissions Program

IV. Summary of 1989 NPR-1 Tank Inspection Program for KCAPCD Rule 411 
兽

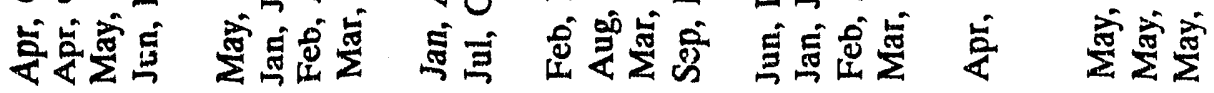

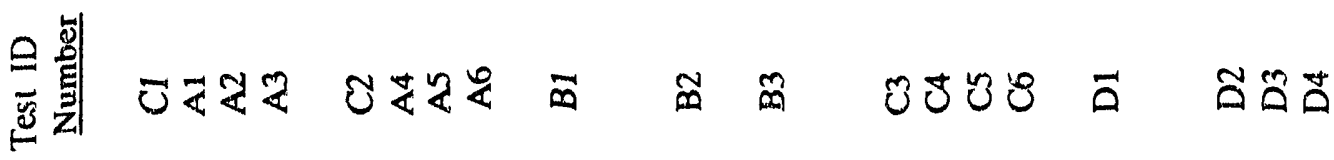

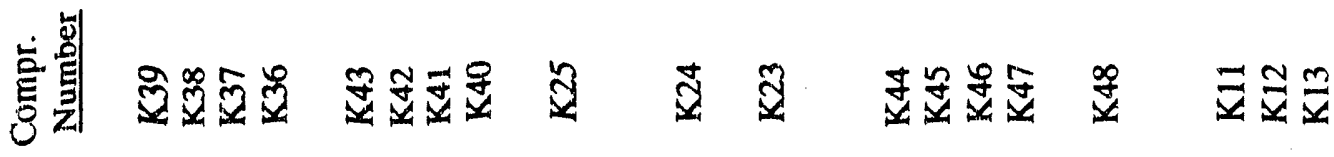

氛

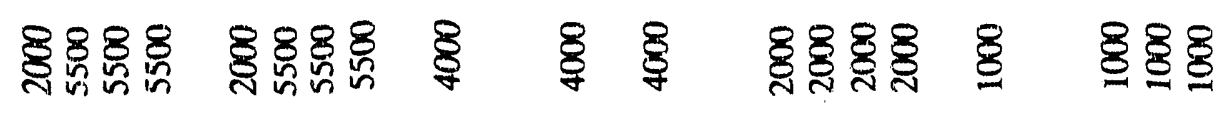

敦

璐诘

$\sum_{10}^{0}$

ㄸํ

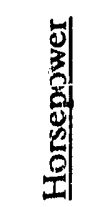

월 용

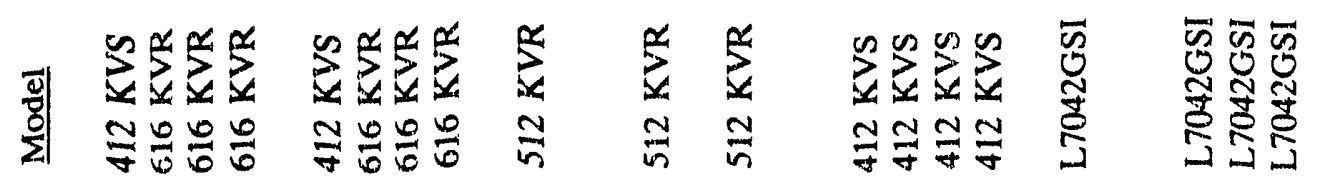

iा

我苛

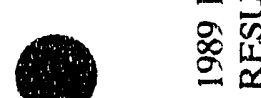

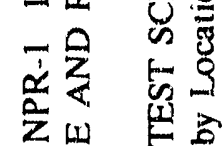

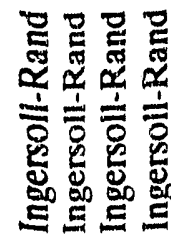

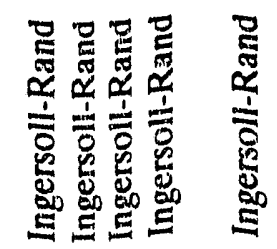

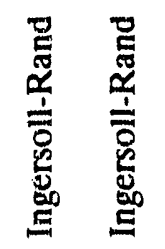

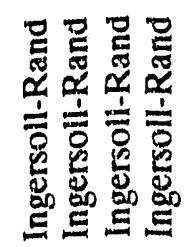

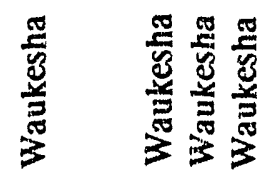

$\sum_{\infty}^{2}$

병

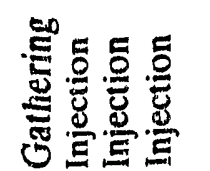

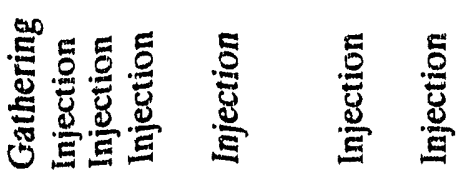

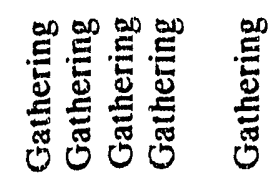

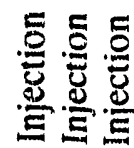

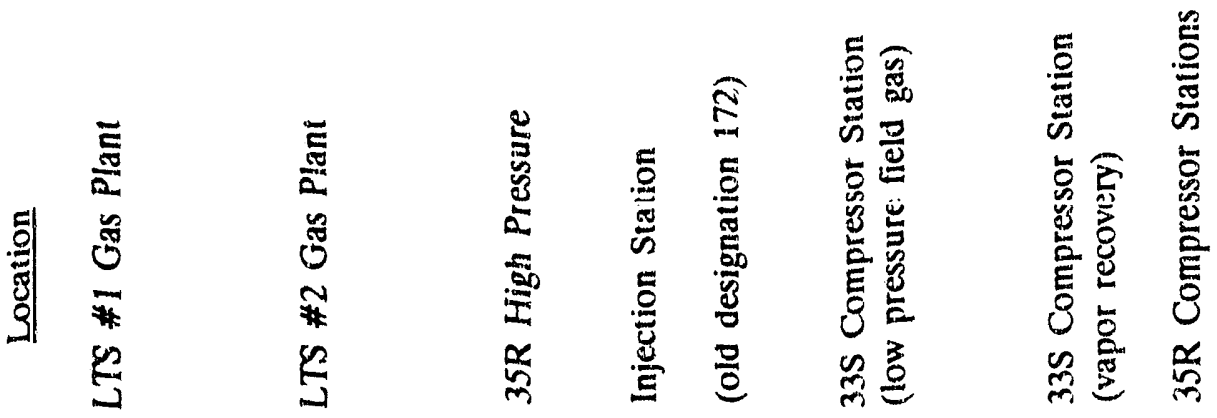




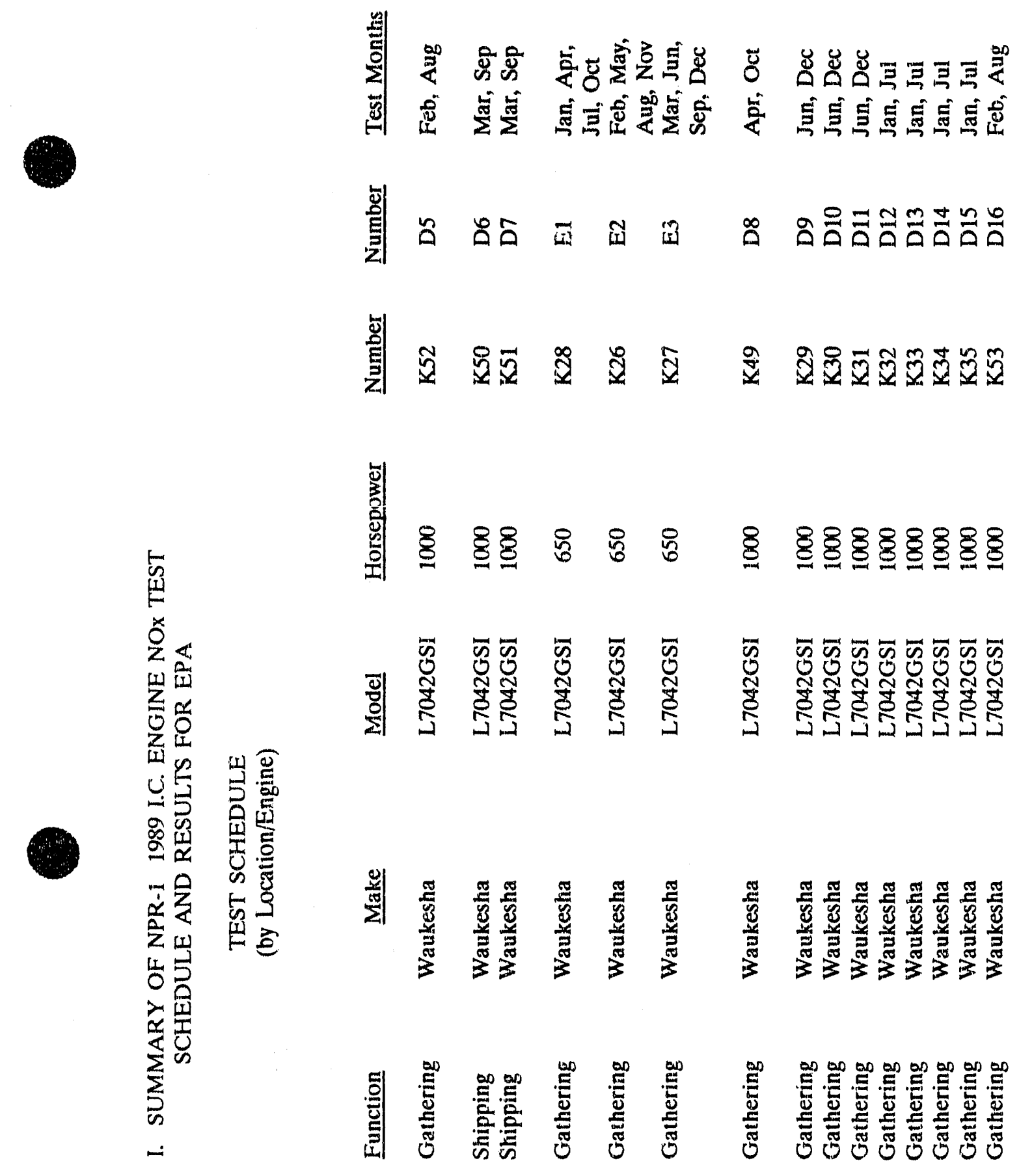


11. maval petrolelu reserve ho.

SUMMARY OO I.C. ENCINE TEST DRTA - 1989

\begin{tabular}{|c|c|c|c|c|c|c|c|c|c|c|}
\hline DATE & $\begin{array}{l}\text { ENGINE } \\
\text { MOn }\end{array}$ & RPM & HP & $\begin{array}{l}\text { FUEL RATE } \\
\text { (HSCF/DAY) }\end{array}$ & $\begin{array}{l}\text { EXMaUST RATE } \\
\text { (OSCFW) }\end{array}$ & $\begin{array}{l}\text { EXPANSION } \\
\text { FACTOR }\end{array}$ & xo? & $\begin{array}{l}\text { MOX } \\
\text { (PPM) }\end{array}$ & $\begin{array}{l}\text { NOX } \\
\text { (LB/HR) }\end{array}$ & $\begin{array}{c}\text { MOX } \\
\text { (GH/BHP-HR) }\end{array}$ \\
\hline \multicolumn{11}{|c|}{ 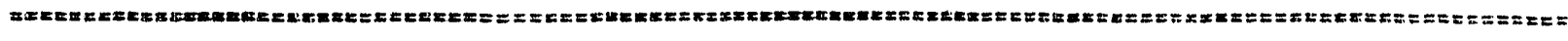 } \\
\hline 04 - Jori-88 & $k-25$ & 342 & 3906 & 429.4 & 13823.00 & 8.571 & 14.7 & 37 & 3.72 & 0.42 \\
\hline $04-$ Jan-89 & $k-42$ & 315 & 3611 & 308.0 & 9729.00 & 9.392 & 14.7 & 199 & 13.53 & 1.70 \\
\hline $04-\tan -80$ & $x-35$ & 920 & 742 & 153.8 & 2653.00 & 10.962 & 7.6 & 785 & 15.16 & 9.27 \\
\hline $04-\operatorname{dan}-89$ & $x-34$ & 960 & $2 x$ & 198.8 & 1975. & 10.982 & 7.1 & 676 & 9.72 & 4.92 \\
\hline $04-J a n-80$ & $x-33$ & 937 & 962 & 130.9 & 2192.00 & 10.982 & 7.2 & 552 & 8.81 & 4.16 \\
\hline $04-\operatorname{san}-89$ & $x-32$ & $9 \pi$ & 919 & 160.0 & 2913.00 & 10.922 & 8.3 & 738 & 16.07 & 7.94 \\
\hline $04-J e n-88$ & $k-28$ & 943 & 636 & 78.1 & 10093.00 & 9.512 & 6.7 & 689 & 5.48 & 3.93 \\
\hline $04-\operatorname{Jan}-89$ & $k-45$ & 200 & 2066 & 156.7 & 5197.00 & 0.543 & 14.9 & 216 & 8.17 & 1.81 \\
\hline $07-$ feb-89 & $k-24$ & 34,3 & 3880 & 701.4 & $136 \pi 5.00$ & 9.565 & 13.9 & 41 & 4.08 & 0.48 \\
\hline 07 -Feb- 89 & $k-39$ & 317 & itrs & 209.5 & 3979.00 & 9.565 & 11.9 & 182 & 4.21 & 1.08 \\
\hline $07-$ feb- 89 & $x-61$ & 350 & 5228 & $\pi .2$ & 13720.00 & 9.627 & 13.3 & 93 & 9.28 & 0.81 \\
\hline $07-F e b-89$ & $x-53$ & 950 & 1089 & 175.5 & 1778.00 & 10.146 & 6.6 & 775 & 10.03 & 4.18 \\
\hline $07-$ Feb- 89 & $k-26$ & 844 & 628 & 111.3 & 1068.00 & 9.336 & 7.0 & 371 & 2.88 & 2.08 \\
\hline $07 \cdot$ feb- 89 & $x-52$ & 950 & 909 & 93.6 & 709.00 & 8.276 & 0.5 & 583 & 3.26 & 1.63 \\
\hline $23-\operatorname{Mar}-89$ & $k-12$ & 986 & 1122 & 328.3 & 3642.00 & 9.686 & 7.7 & 481 & 12.04 & 6.87 \\
\hline 23 - Mer - 89 & $k-23$ & 305 & 3985 & 615.0 & 10343.00 & 9.544 & 12.8 & 202 & 15.19 & 1.73 \\
\hline 23 -Mar-89 & $k-40$ & 346 & 4999 & 802.5 & 1513.4 .00 & 9.385 & 13.8 & 85 & 9.36 & 0.85 \\
\hline 23 -Mar-89 & $k-47$ & 325 & 1829 & 396.8 & 5003.00 & 9.514 & 12.3 & 54 & 1.96 & 0.48 \\
\hline 23 -Mer -89 & $x=50$ & 936 & 1101 & 284.0 & 3735.00 & 11.200 & 8.8 & 529 & 14.45 & 5.96 \\
\hline $06-A p r-89$ & $x-25$ & 348 & 4026 & 647.9 & 11378.00 & 9.599 & 13.1 & 109 & 9.02 & 1.02 \\
\hline$O 6-A p r-8 Q$ & $x-27$ & 879 & 573 & 132.6 & 1233.00 & 9.498 & 6.3 & 563 & 4.87 & 3.86 \\
\hline OS-Apr-BQ & $k-2 B$ & 900 & 585 & 110.9 & 1038.00 & 9.498 & 6.4 & 870 & 0.57 & 5.10 \\
\hline Ob-Apr-BQ & $k-38$ & 350 & 5122 & 851.200 & 21177.00 & 9.599 & 15.4 & 57 & 8.78 & 0.78 \\
\hline $06-A p r-89$ & $k-46$ & 330 & 2086 & 329.4 & $50 \% .00$ & 9.612 & 12.0 & 42 & 1.55 & 0.34 \\
\hline 06 -Apr-89 & $k=49$ & 873 & 933 & 192.0 & 2003.00 & 9.498 & 7.9 & 128 & 1.06 & 0.91 \\
\hline $04-$ - Мау -89 & $k-11$ & 925 & 953 & 293.0 & 3065.00 & 9.810 & 7.5 & 358 & 7.98 & 3.80 \\
\hline O4-May-89 & $x-13$ & 906 & 876 & 180.0 & 1843.00 & 9.810 & 7.5 & 640 & 6.03 & 3.12 \\
\hline $00_{6}-\mathrm{May}_{0}-89$ & $x-26$ & 350 & 3766 & 656.0 & 11580.00 & 9.526 & 13.2 & 56 & 4.72 & 0.57 \\
\hline $04-$ May -89 & $x-26$ & 896 & 556 & 102.3 & 1017.00 & 9.541 & 7.2 & 196 & 1.45 & 1.18 \\
\hline $06-$ - & $x-37$ & 346 & 5178 & 668.3 & 11645.00 & 9.526 & 13.1 & 140 & 11.86 & 1.06 \\
\hline 04 -Moy- $B 9$ & $x-63$ & 320 & 2002 & 325.8 & 4094.00 & 9.387 & 11.6 & 117 & 3.90 & 0.87 \\
\hline $08-\operatorname{sun}-89$ & $k-23$ & 350 & 3461 & 680.4 & $123 \% .00$ & 9.648 & 13.3 & 124 & 11.18 & 1.48 \\
\hline $08-\operatorname{din}-89$ & $k \cdot 36$ & 350 & 5236 & 818.2 & $1528 x .00$ & 9.648 & 13.5 & 105 & 19.67 & 1.01 \\
\hline $08-\sin -89$ & $k-28$ & 975 & 623 & 121.0 & 1126.00 & 0.634 & 6.1 & 703 & 5.76 & 4.20 \\
\hline $08-\operatorname{Jun}-89$ & $k-30$ & 812 & 629 & 163.5 & 1764.00 & 9.525 & 8.1 & 364 & 4.62 & 3.33 \\
\hline $08-J u n-88$ & $x-32$ & 980 & 979 & 157.5 & 1004.00 & 9.525 & 7.5 & 600 & 7.00 & 3.25 \\
\hline $08-\sin -8 \theta$ & $x-33$ & 955 & 1163 & 220.7 & 2345.00 & 9.525 & 7.6 & 676 & 11.53 & 4.50 \\
\hline $08-d u n-89$ & $x-64$ & 302 & 22008 & 297.0 & 5233.00 & 11.216 & 11.8 & 184 & 7.00 & 1.64 \\
\hline $20-J u l-89$ & $x-25$ & 348 & 3518 & 639.1 & 10850.00 & 0.635 & 12.8 & 193 & 15.23 & 1.97 \\
\hline $20-J u l-89$ & $k-27$ & 936 & 602 & 133.3 & 1236.00 & 9.515 & 6.2 & 656 & 5.90 & 4.45 \\
\hline $20-J u l-80$ & $x-20$ & 846 & 486 & 126.0 & 1387.00 & 9.531 & 8.5 & 508 & 5.12 & 4.70 \\
\hline $20-J u l \cdot 89$ & $k-39$ & 955 & 1236 & 219.6 & 2267.00 & 9.531 & 7.7 & 231 & 3.81 & 1.40 \\
\hline $20-201 \cdot 89$ & $k-36$ & 05.4 & oas & 195.6 & 1823.00 & 9.531 & 6.3 & 878 & 19.64 & 5.85 \\
\hline
\end{tabular}


$02 \cdot A p r-90$

11. MAVAL PETROLEUM RESERVE NO. 1

SUMAARY OF I.C. ENGINE TEST DATA 1989

\begin{tabular}{|c|c|c|c|c|c|c|c|c|c|c|}
\hline DATE & $\begin{array}{l}\text { EMGINE } \\
\text { WO. }\end{array}$ & RPM & HP & $\begin{array}{r}\text { FUEL RATE } \\
\text { (ASCF/OAY) }\end{array}$ & $\begin{array}{l}\text { EXMaUST RATE } \\
\text { (DSCFM) }\end{array}$ & $\begin{array}{l}\text { Expansion } \\
\text { FACTOR }\end{array}$ & $x 02$ & $\begin{array}{l}\text { Max } \\
\text { (PPM) }\end{array}$ & $\begin{array}{c}\text { NOX } \\
\text { (LE/HR) }\end{array}$ & $\begin{array}{c}\text { NOX } \\
\text { (GM/BHP- HR) }\end{array}$ \\
\hline \multicolumn{11}{|c|}{ 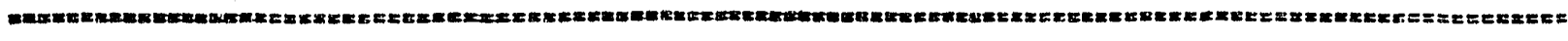 } \\
\hline 20- Jul -89 & $x-35$ & 975 & 786 & 176.3 & 1820.00 & 9.531 & 7.7 & 260 & 3.44 & 1.99 \\
\hline 20- dul-80 & $x-45$ & 318 & 1966 & 294.8 & 5683.00 & 9.678 & 14.0 & 119 & 5.00 & 9.18 \\
\hline $27-J u t-89$ & $k-49$ & 365 & 5341 & 955.9 & 15954.00 & 9.572 & 12.9 & 159 & 18.45 & 1.57 \\
\hline 20-Aug-89 & $x-24$ & $x$ & $2 ? ! 4$ & 502.0 & จิ17̄ó.00 & 9.572 & 13.4 & 26 & 1.74 & 0.29 \\
\hline $20-$ Aug - 89 & $k-26$ & 907 & 592 & 116.0 & 1067.00 & 9.437 & 6.5 & 859 & 6.67 & 5.11 \\
\hline $20-A u g-89$ & $k-42$ & 348 & 4793 & 524.0 & 9712.00 & 9.461 & 13.5 & 75 & 5.30 & 0.50 \\
\hline $20-A c 00-89$ & $k=42$ & 350 & 5696 & 601.5 & 10907.00 & 9.458 & 13.5 & 91 & 7.22 & 0.58 \\
\hline $20-A \log -89$ & $k-46$ & 300 & 1798 & 305.0 & 6708.00 & 9.535 & 12.0 & 71 & 2.43 & .0 .61 \\
\hline $20-\operatorname{Aug}-89$ & $k-52$ & 944 & 897 & 207.0 & 1907.00 & 8.237 & 8.1 & 458 & 2.19 & 1.19 \\
\hline $20-\operatorname{Aug}-89$ & $k-53$ & 933 & 1198 & 235.0 & 2800.00 & 9.480 & 9.1 & 4402 & 9.00 & 3.42 \\
\hline $20-\operatorname{sep}-89$ & $x-23$ & 348 & 3283 & 606.8 & 11997.00 & 9.683 & 13.9 & 127 & 11.08 & 1.53 \\
\hline $20-\operatorname{sep}-89$ & $k-27$ & 910 & 578 & 128.7 & 1209.00 & 9.509 & 6.4 & 790 & 6.95 & 5.46 \\
\hline $20-\operatorname{sep}-89$ & $x-40$ & 347 & 5088 & 744.0 & 15287.00 & 9.529 & 14.3 & 35 & 3.89 & 0.35 \\
\hline $20-$ Sep-89 & $k-47$ & 300 & 1506 & 221.8 & 3503.00 & 9.561 & 12.3 & 53 & 1.35 & 0.41 \\
\hline $20-\operatorname{sep}-89$ & $x-50$ & 860 & 852 & 215.0 & 1904.00 & 8.271 & 7.6 & 621 & 8.60 & 4.58 \\
\hline $20-\operatorname{sep}-89$ & $k-51$ & 962 & 996 & 243.2 & 2093.00 & 8.271 & 7.2 & 781 & 11.89 & 5.42 \\
\hline $20-$ Sep- 89 & $k-52$ & 863 & 863 & 529.3 & 4.648 .00 & 8.271 & 7.4 & 286 & 9.67 & 5.00 \\
\hline $20-\cot -89$ & $k-28$ & .860 & 629 & 101.6 & 1039.00 & 9.509 & 7.6 & 938 & 7.09 & 5.12 \\
\hline $20-0<t-80$ & $k-38$ & 350 & 4735 & 865.0 & 17590.00 & 10.712 & 13.4 & 150 & 19.18 & 1.84 \\
\hline $20-0<t-89$ & $x-39$ & $\mathbf{3 3 0}$ & 2003 & 267.3 & 4439.00 & 90.712 & 11.7 & 171 & 5.52 & 1.25 \\
\hline $20-0 c t-89$ & 6.69 & 245 & 916 & 203.8 & 2119.00 & 9.500 & 7.8 & 438 & 6.75 & 3.35 \\
\hline $20-0 c t-89$ & $x-59$ & 964 & 1080 & 218.8 & 2222.00 & 8.271 & 9.5 & 194 & 1.84 & 0.77 \\
\hline $20-0 c t-89$ & $x-25$ & 350 & 3091 & $78 B .5$ & 14916.00 & 9.590 & 13.7 & 115 & 12.47 & 1.83 \\
\hline 16-Wor-89 & $k-11$ & 925 & 919 & 345.6 & 3387.00 & 9.629 & 6.9 & 372 & 9.16 & 4.53 \\
\hline 16 -Mov-89 & $x-12$ & 954 & 761 & 369.0 & 3900.00 & 9.629 & 7.9 & 218 & 6.18 & 3.69 \\
\hline 16-Nov-89 & $x-13$ & 900 & 869 & 177.4 & 1766.00 & 0.629 & 6.9 & 772 & 9.80 & 5.12 \\
\hline 16-Nov-89 & $k-24$ & 350 & 4328 & 743.8 & 11666.00 & 9.620 & 12.5 & 63 & 5.35 & 0.56 \\
\hline 16-Mov-89 & $x-26$ & 933 & 636 & 110.2 & 1874.00 & 10.885 & 6.3 & 678 & 5.79 & 4.13 \\
\hline 16-Nov-89 & $x-37$ & 364 & 4201 & 674.7 & 8022.00 & 0.629 & 12.8 & 255 & 14.88 & 1.61 \\
\hline 16-Mor-89 & $k-43$ & 320 & 20086 & 321.8 & 6673.00 & 9.466 & 18.6 & 97 & 3.30 & 0.72 \\
\hline
\end{tabular}

01-DeC-89 MOMIMLY NOX SOURCE TESTING CANCELED / AMMLAL PERMIT RENEUAL SOURCE TESTING PREFORMED. 


\section{Summary of NPR-1 KCAPCD Rule 414.7 \\ Fugitive Emission Program}

Following is a report on the inspection program for $1989^{*}$

Number of Components Inspected

177,070

Number of Leaks** Found

930

Number of Leaks** Repaired by F.E.

309

Number of Leaks** Needing Repairs \& Tagged

435

Number of Leaks** Repaired, Reinspected, and Tags Removed

* The program at present is a "fix-it" program rather than a "leak-is-a-violation" program. The data will be used to establish an allowed frequency of leaks. The violation will occur if the KCAPCD inspection finds more leaks than the allowed leak rate.

** Leak is defined by Rule 414.7 as 10,000 ppm or greater, excluding methane, or more than three (3) drops per minute of liquid containing volatile organic compounds. 


\title{
IV. Summary 1989 NPR-1 Tank Inspection Program for KCAPCD Rule 411
}

\section{Attachment}

\author{
Table of Contents
}

No.

1. Summary of leaking components greater than $10,000 \mathrm{ppm}$ non-methane hydrocarbons (NMHC).

2. Summary of leaking components for 1989 (percent contribution of each component type to the total number of leaking components $>10,000$ NMHC). 


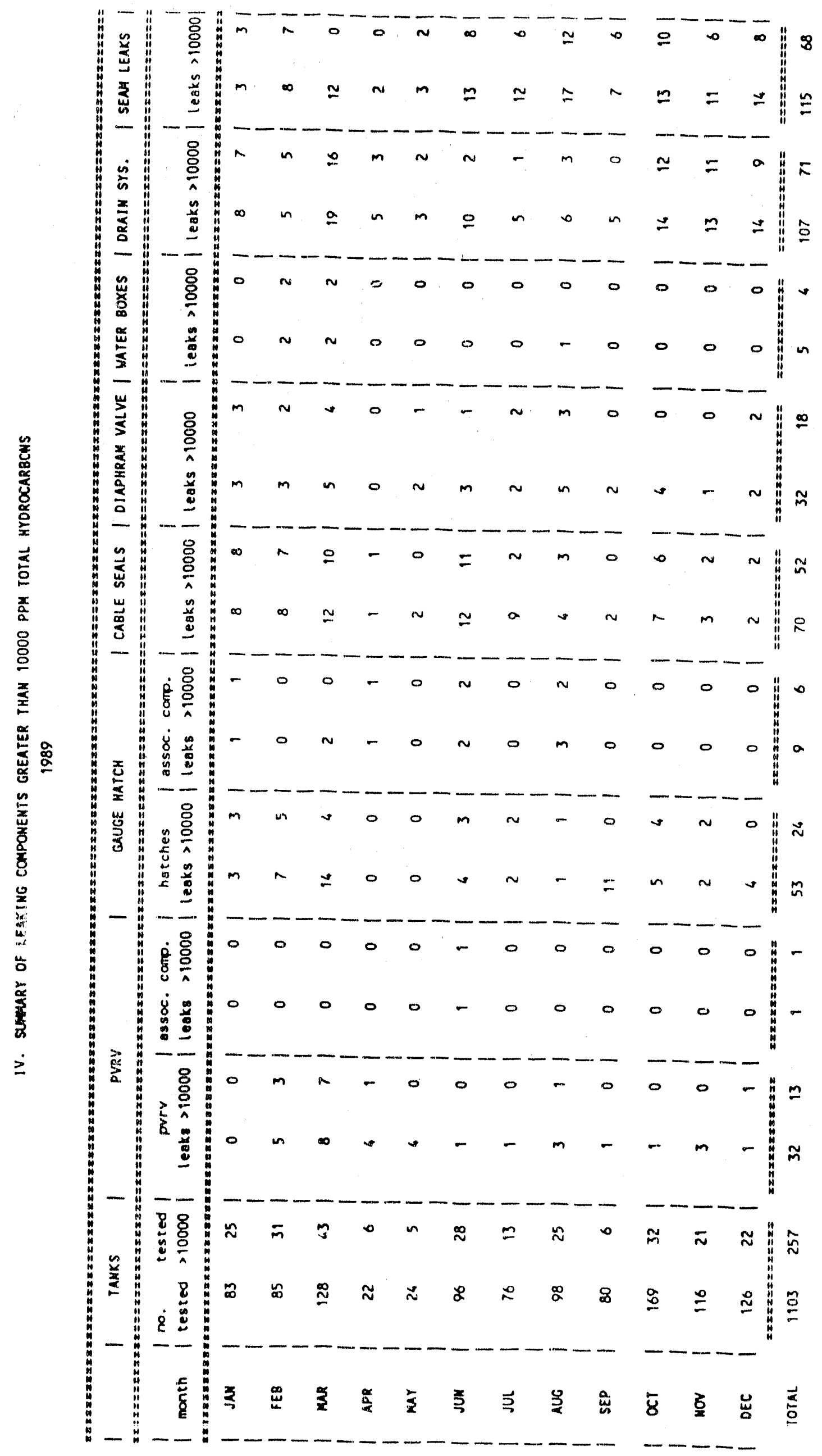


SU WIARY OF LEAKING COMPONENTS FOR 1989

(PERCENT CONYRIBUTION OF EACH COMPONENT TYPE TO THE TOTAL

HUMBER OF L.EAKIMG COMPONENTS GREATER THAN 10000 PPM TOTAL. HYDROCARBONS).

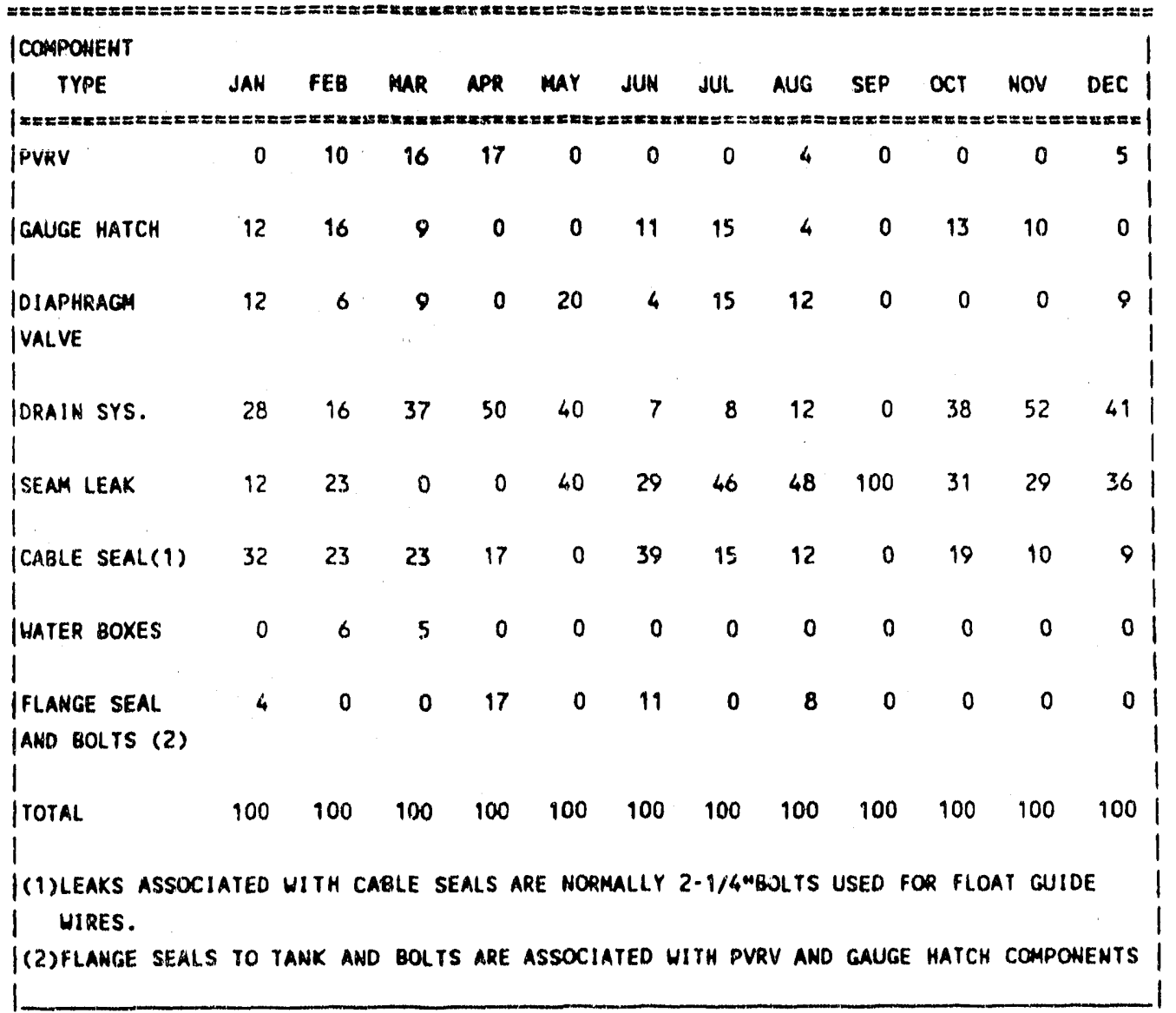




\section{APPENDIX D}

AIR MONITORING DATA FOR 1989 FROM WEST SAN JOAQUIN MONITORING GROUP (BPOI MEMBER) 


\section{WEST SAN JOAQUIN MONITORING GROUP}

\section{AIR QUALITY DATA FOR 1989}

See Figure 1-1 attached for site location

\section{Number of Exceedances 1989}

$\begin{array}{ccccc}\text { Maricopa } & \text { Fellows } & \text { McKittrick } & \text { Kernridge } & \text { Kem Widlife } \\ - & - & - & - & 6 \\ 13 & - & - & - & - \\ - & - & - & - & - \\ - & 20 & - & - & 17\end{array}$

${ }^{1}$ Beginning 1/1/89.

${ }^{2}$ Federal secondary standard.

${ }^{3}$ Federal primary standard.

${ }^{4}$ Ozone exceedances are reported in pphm (parts per hundred million). Only one exceedance per day is counted. 


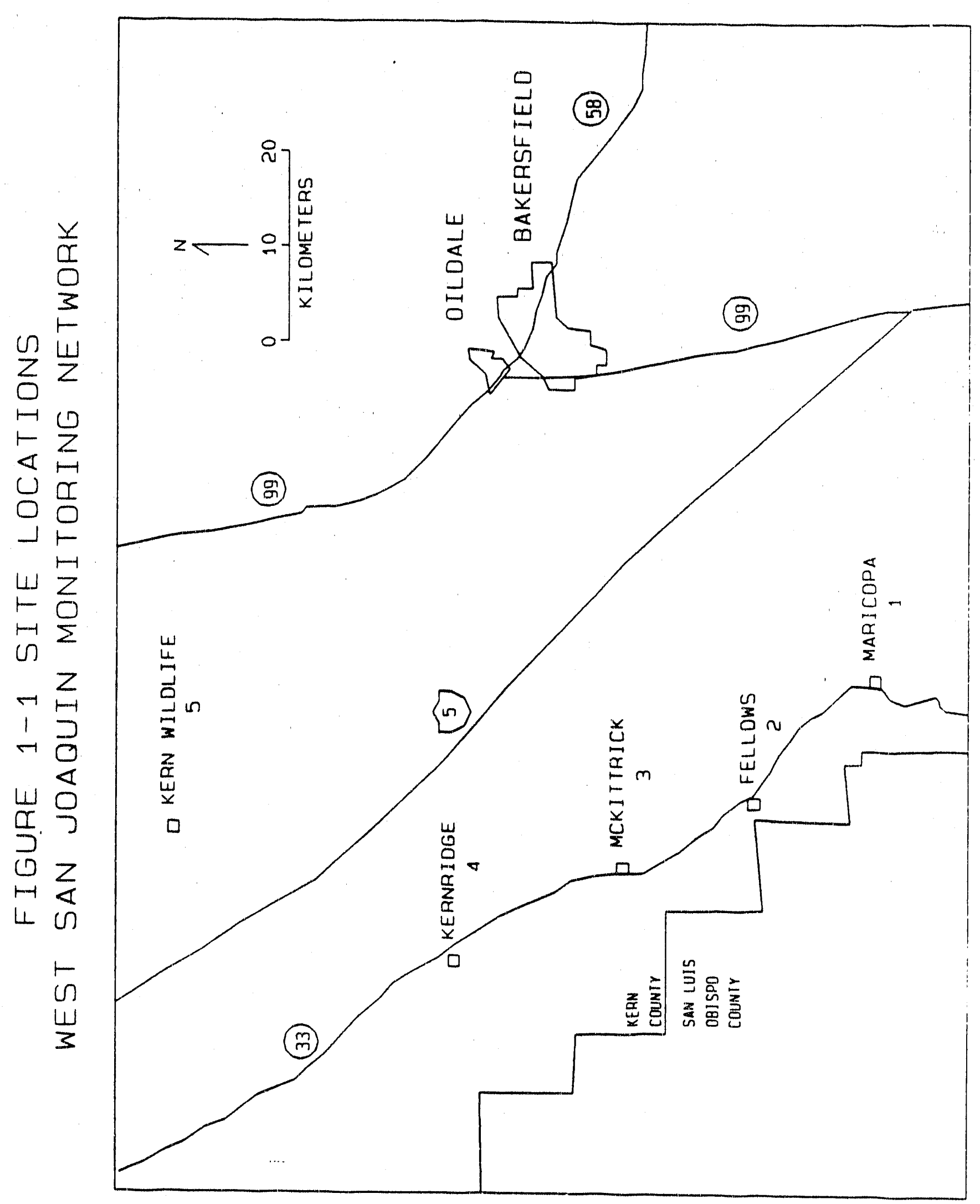




\section{APPENDIX E}

SUMMARY OF NPR-1 OIL SPILLS AND CORRECTIVE ACTION EVALUATION FOR 1989 


\section{TABLE OF CONTENTS}

\section{APPENDIX E}

Attachment I - Total Spills by Cause

Attachment I shows that 5307 barrels of fluid were spilled in 580 separate incidents; 3826 barrels $(72 \%)$ were recovered and 1447 barrels having an estimated value of $\$ 25,268$ were not recovered. Minor oil spills (less than 100 barrels each) accounted for 2237 barrels (42\%) and major oil spills (100 or greater barrels) accounted for 3070 barrels (58\%) of the total volume spilled. At least $89 \%$ of the fluid spilled in each major incident was recovered, and total recovery of fluids for all 1989 major spills was $97 \%$.

Attachment II - 1989 Minor Oil Spills

Attachment II shows that $44 \%$ of the total number of minor oil spills in 1989 were due to corrosion, and $32 \%$ were due to mechanical causes.

Atiachment III - Barrels of Oil Spilled - 1989

Attachment III depicts the total number of barrels spilled for each cause category, including minor and major spills. Of the total volume, 2075 barrels (39\%) were the result of corrosion spills, while 2816 barrels (53) were mechanically-related.

Attachment IV - Significant Corrosion Related Spills

Attachment IV identifies the sixteen (16) facilities/sources of the most significant corrosionrelated oil spills. The $28 \mathrm{~S} \mathrm{SOZ}$ gravity line accounted for 23 (9\%) and 756 barrels (25\%) of all corrosion-related oil spills.

Attachment V - Significant Mechanical Related Spills

Attachment $\mathrm{V}$ identifies the twelve (12) facilities/sources of the most significant mechanically-related oil spills. The 1-26Z Asphalto Tank Setting accounted for largest number of occurrences (14), due to mechnnical problems in 1989.

Attachment VI - Oil Spills During 1989

Attachment VI lists all 1989 oil spills showing date, time, location, source, volume, and cause for each. 


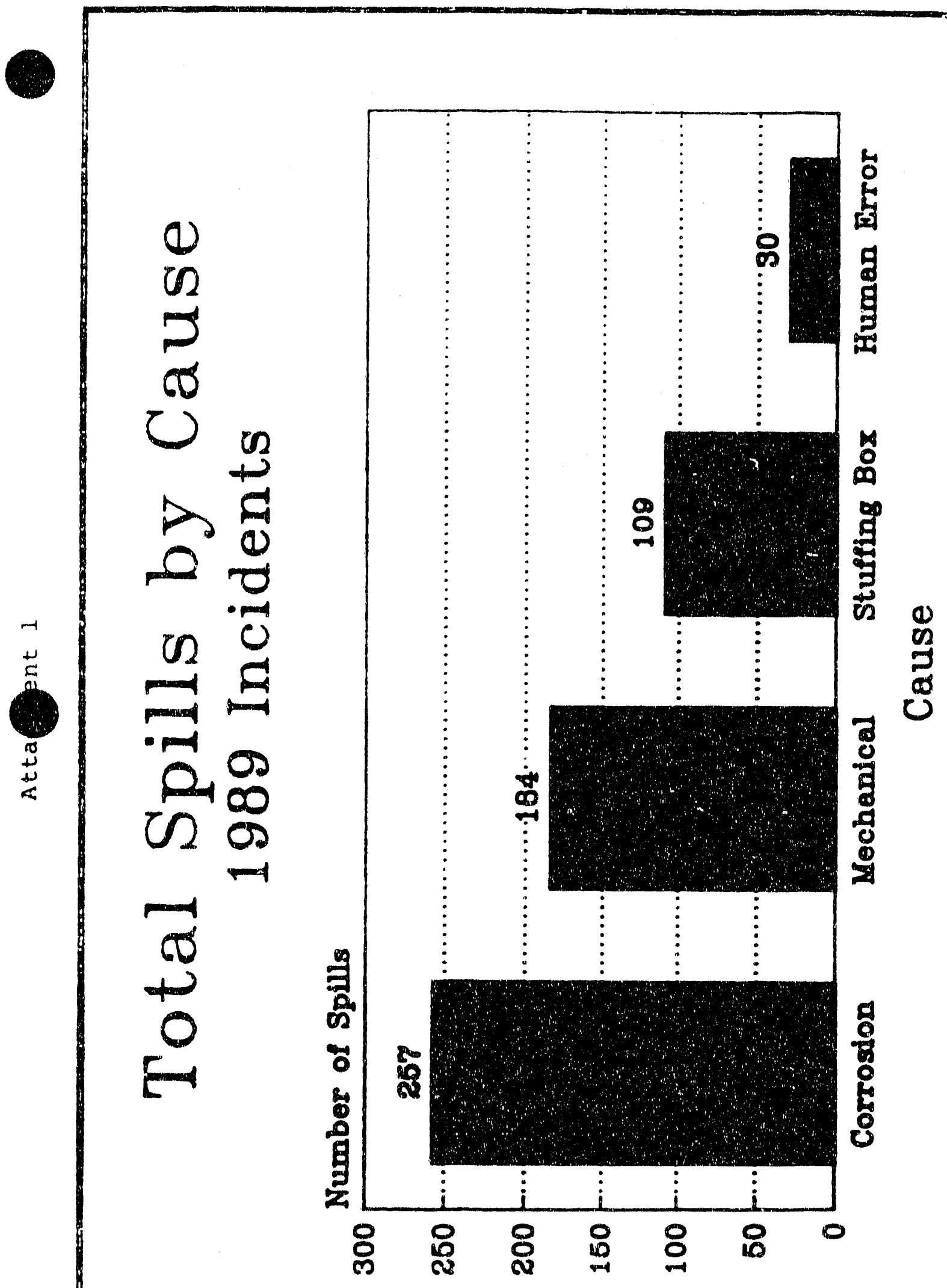




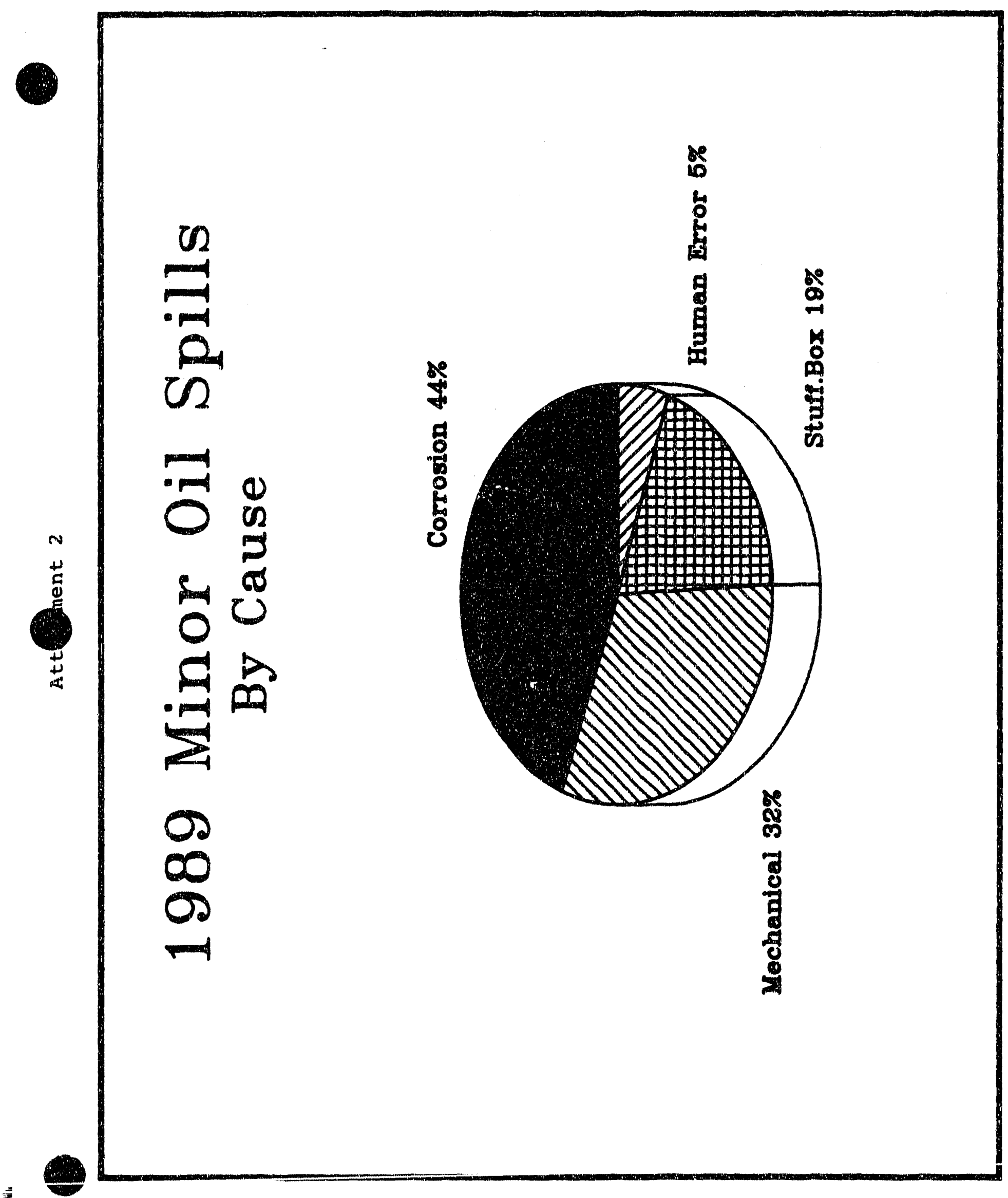




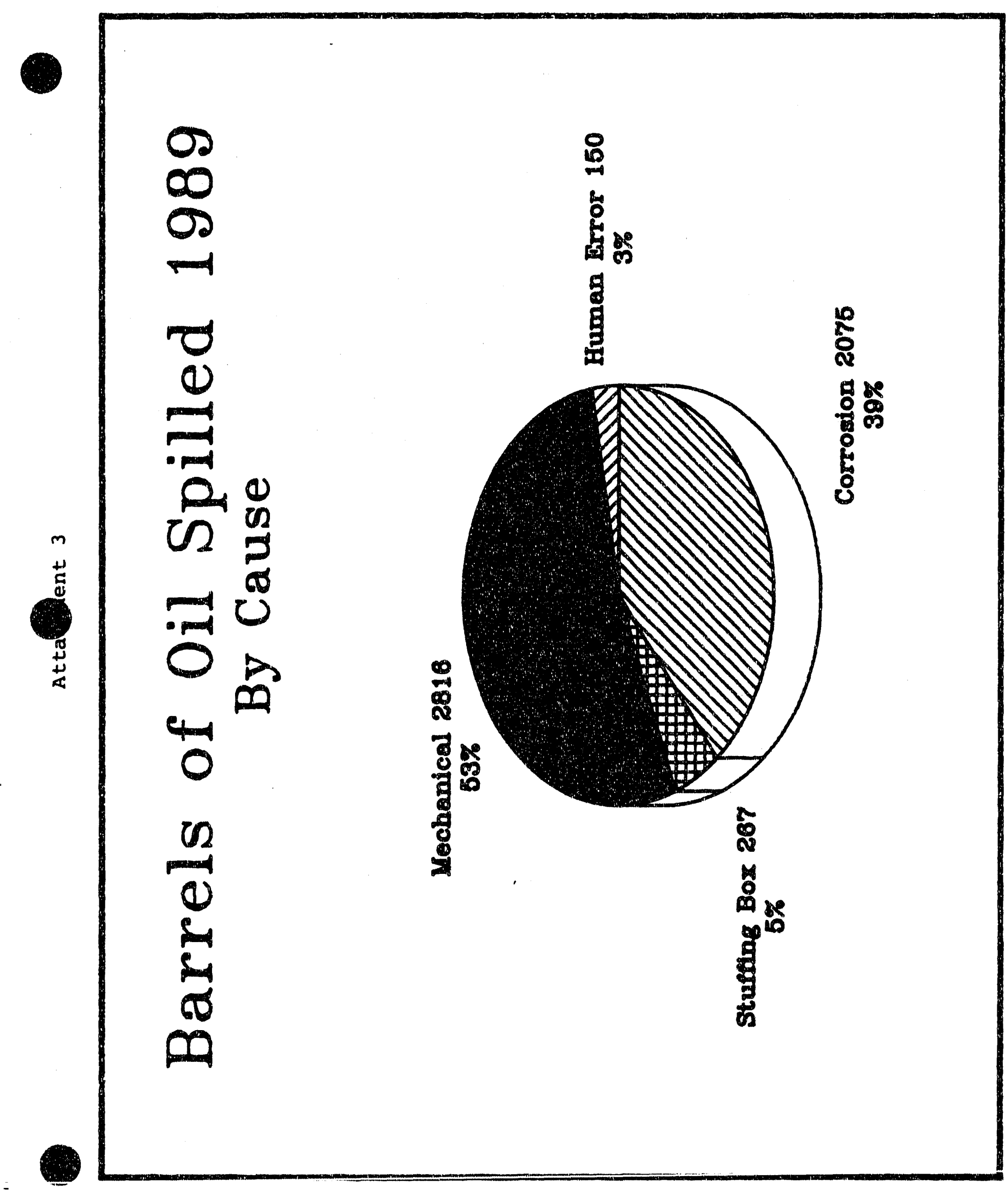




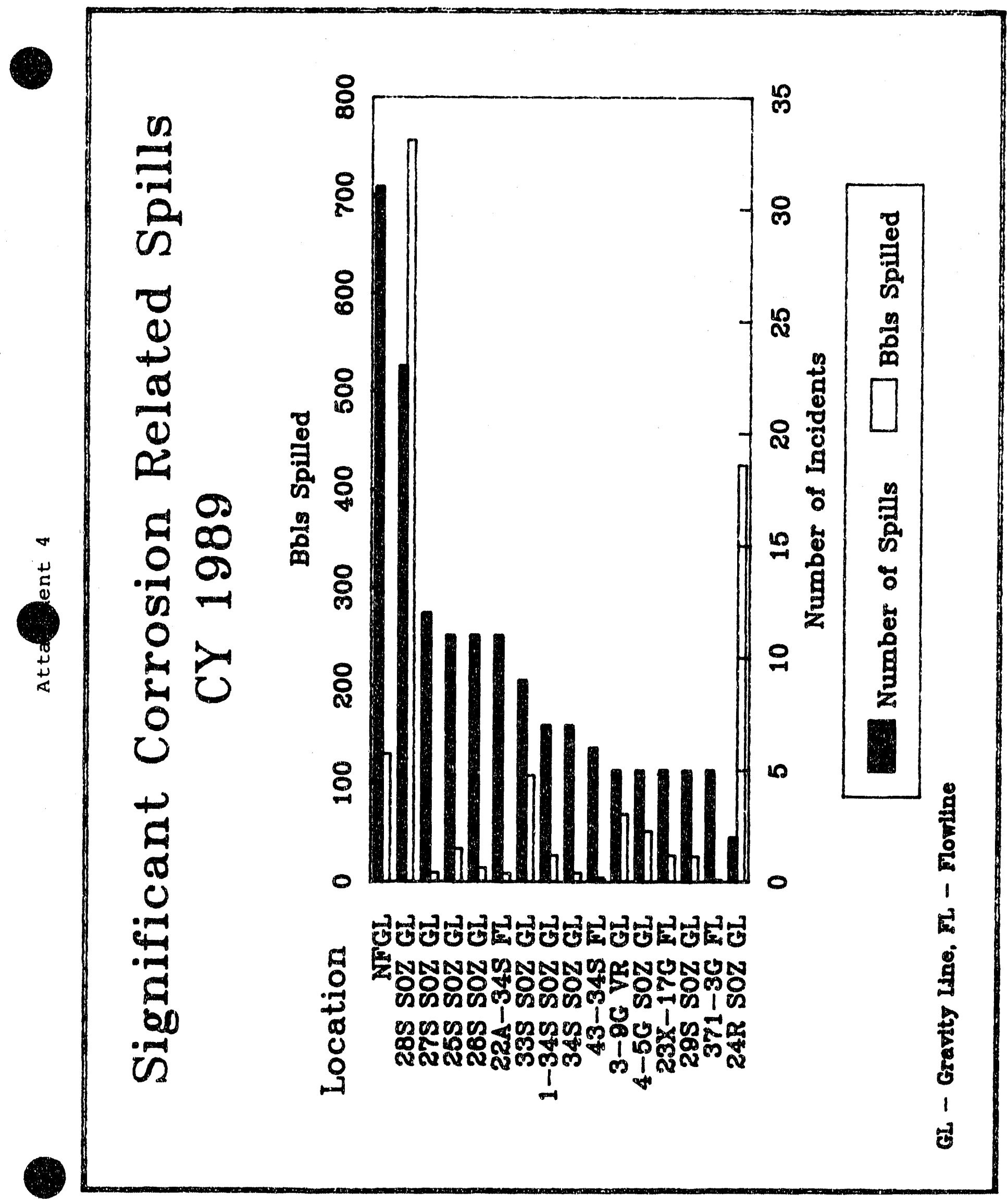




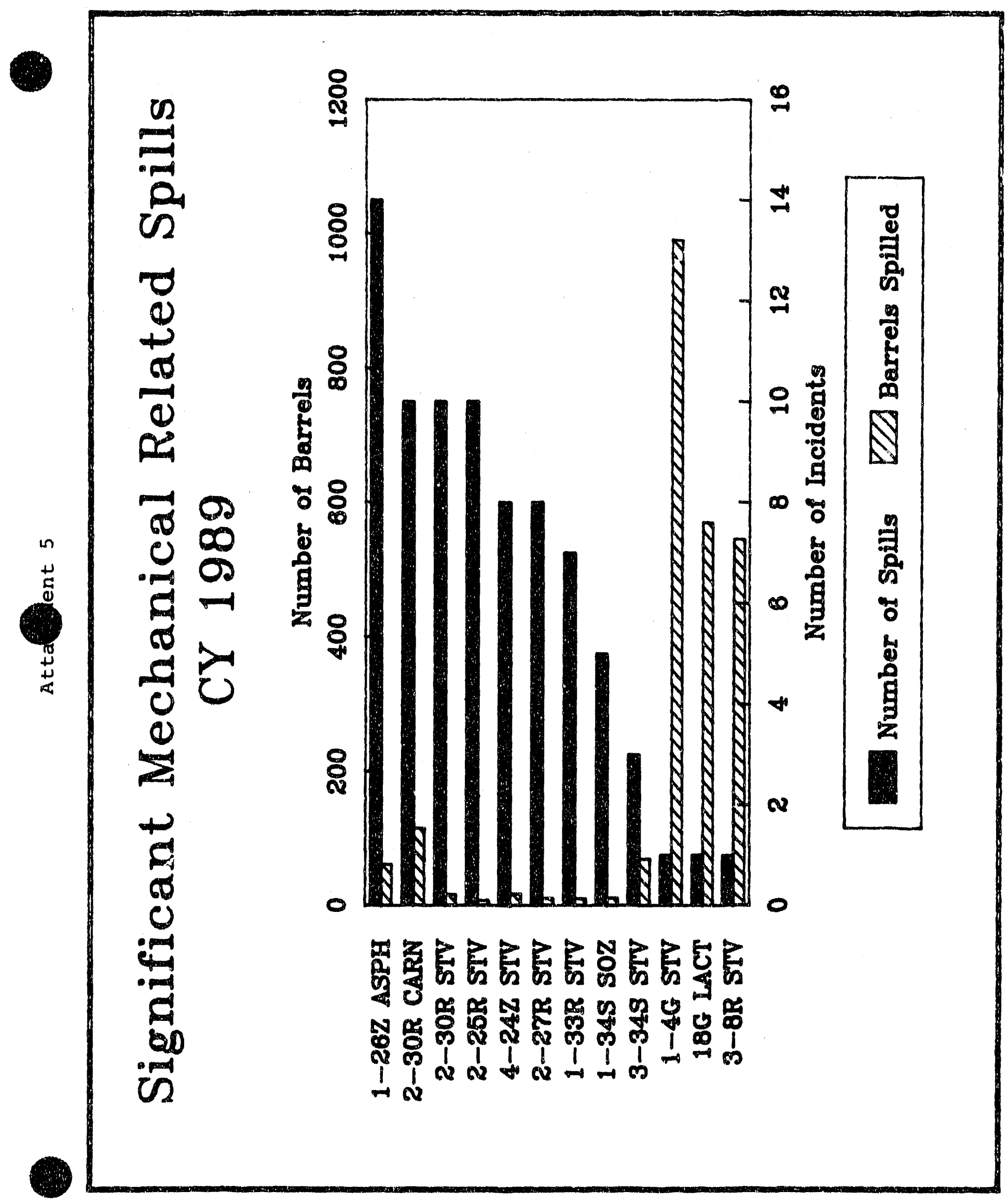




\section{Attachment 6}

OIL EPILLS DURIMG 1900

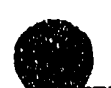
Cloanup

Rpt

Estimted Aarrels cost Eat.

Date Mo. Time sect. Source of splll spllled Recovered in 8

Besic Cause.

Action to

Prevent Recurrence

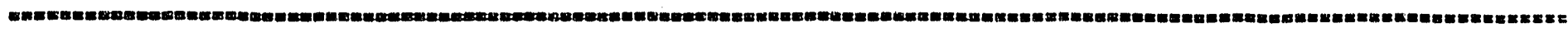

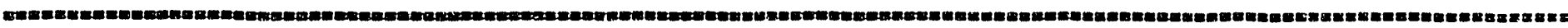

Cleanup

Rpt Eetionted sarrols Coet Eat.

Dete Ho. TIne sect. Source of splll spllled hecovered In 5 Prevent Recurrence

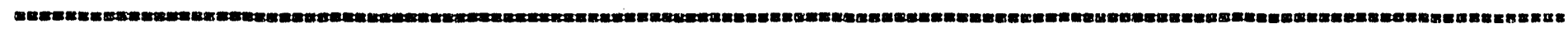

\begin{tabular}{|c|c|c|c|c|}
\hline $3 / 20$ & 106 & 1000 & 278 & 198-27s flow line \\
\hline $12 / 10$ & 534 & 9000 & 26 & 19f-26 flow line \\
\hline $1 / 21$ & 20 & 0000 & 106 & 19-106 flow line \\
\hline $10 / 2$ & 426 & 1900 & 176 & 11-17g flow Line \\
\hline $3 / 13$ & 101 & 0700 & 18 & 11-18 flow line \\
\hline $5 / 31$ & 214 & 0800 & 138 & $1383^{*}$ Grovity line \\
\hline $4 / 3$ & 129 & 0230 & 16 & 13-16 flow Line \\
\hline $5 / 20$ & 201 & 1000 & $25 \$$ & 16" 502 Gravity line \\
\hline $1 / 24$ & 25 & 1000 & 285 & 17-285 flow line \\
\hline $7 / 6$ & 269 & 900 & $28 s$ & 17-285 flow line \\
\hline $12 / 16$ & 548 & 0600 & 186 & 180 LACT Shipping Punp \\
\hline $10 / 6$ & 435 & 1430 & 10 & IA-1G 502 Gravity line \\
\hline $7 / 29$ & 321 & 1930 & 126 & 1-126 soz Grovity line \\
\hline & 114 & 1730 & 345 & 1-34s soz Grevity Lime \\
\hline & 110 & 1600 & 345 & 1-34s saz Grevity line \\
\hline $4 / 17$ & 149 & 0930 & $34 s$ & 1-36s soz Grevity line \\
\hline $10 / 6$ & 436 & 2100 & 345 & 1-345 soz Grevity line \\
\hline $4 / 3$ & 128 & 1100 & 345 & 1-36s soz Grevity Line \\
\hline $7 / 2$ & 263 & 1030 & 365 & 1-348 soz Grevity line \\
\hline $5 / 5$ & 170 & 9000 & 345 & 1-36s 502 Grevity Line \\
\hline
\end{tabular}

12
3
8
7
11
2
0.06
0.125
7
7
2
5
3

6185

195

3105

0110

140

140

90

0

9

200

90

105

95

90

2
4
3
0.5
7.5
8
2

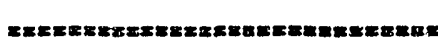

$27 \quad 18$

497

$11 / 24517 \quad 1130 \quad 3459-345502$ Pool Line

$2 / 10 \quad 4817003451-345$ sTV Grovity line

12/21 5610030 36s 1-36s s02 unten flow line

$11 / 13507 \quad 0900$ 35s 206-35s flow line

$7 / 62669200 \quad 345$ 21A-34s flow line

$11 / 4 \quad 4950930 \quad 345 \quad 224345$ flow line

$12 / 175651000 \quad 345 \quad 22 A-345$ flow line

$11 / 28522 \quad 1130$ 34s $224-345$ flat line

$12 / 27 \quad 576 \quad 1245 \quad 345 \quad 22 A-345$ flow line

$12 / 145390930$ 345 22A-34s flow line

$1 / 31 \quad 36 \quad 1000 \quad 345 \quad 22 A-3 / 45$ flow line

$11 / 3494 \quad 1030 \quad 345 \quad 22 A-345$ flow line

$12 / 18553 \quad 1100 \quad 34 \mathrm{~S} \quad 22 \mathrm{~A}-34 \mathrm{~S}$ flow line

$10 / 26475 \quad 1130 \quad 345 \quad 22 A-345$ flow line

o $4170830345 \quad 22 A-345$ flowline
Internal corrasion Internal corrosion Internal corrosion Internal corrosion Internal corrosion Internal corrosion internal corrosion Internal corrosion Internal corrosion Internal Corrosion Internal Corrosion Internal corrosion Internal corrosion

(1) Clemped line.

(1) Clapod line

(1) Clemped líne.

(1) Clapoed line

(I) Claped IIne.

(1) cleaped leak.

(1) clapped line.

(I) Clemped loak.

(1) Clapped IIne.

(1) Clamed line

(1) Replaced Line

(1) Clamped line

(1) Clemped Line

(1) Clauped line.

(1) Clamped line.

(1) clasped line.

(y) clapped line

(1) Cleaped tíne.

(1) Clemped Line

(1) Clemped leak.

$\begin{array}{rrrl}1 & 0 & 95 & \text { Internal corrosion } \\ 0.5 & 0 & 0 & \text { Internal corrosion } \\ 3 & 0 & 50 & \text { Internal corrocion } \\ 8 & 2 & 140 & \text { Internal corrosion } \\ 1 & 0 & 90 & \text { Internal corrosion }\end{array}$

$\begin{array}{rr}0.25 & 0 \\ 9 & 0 \\ 0.25 & 0 \\ 1 & 0 \\ 3 & 0 \\ 0.125 & 0 \\ 0.125 & 0 \\ 0.125 & 0 \\ 0.125 & 0 \\ 1.5 & 0\end{array}$

Internal Corrosion Internal corrosion Internal corrosion Internal Corrosion Internal Corrosion Internal Corrosion Internal Corrosion Internal Corrosion Internal corrosion Internal corrosion
(1) Clamped Line

(1) clemped line.

(1) Cl umped line

(1) Clapped Line

(1) Cleoped Line

(1) Clemped line

(1) claped line

(1) Clemped line

(1) Clanped line

(1) Clemped Line

(1) Cleaped line.

(1) claped line

(1) Clasped line

(1) Clemed line

(1) Clemped line.

\footnotetext{
- (1) Corrosion (2) Mecmanical faillure (3) STUfFIng roX (4) hUMAH ERror
} 
OIL SPILLS DURING 1909

1 Clearnp

Rpt Estimated Barrels Cost Eot.

Date No. Time Sect. Source of Spill Spllled Recovered In $\$$ Baste Cause

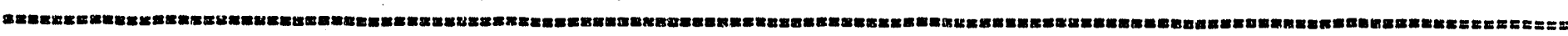

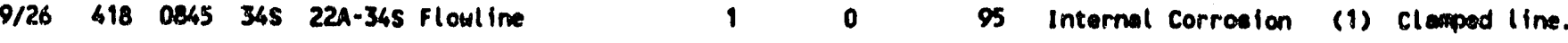

$6 / 5229 \quad 850 \quad 345 \quad 235-348$ Check Volve

$8 / 313681100235$ 23s Grovity line

$10 / 154541500365 \quad 235-365$ Gravity Line

$4 / 4 \quad 130 \quad 1000 \quad 176 \quad 23 x-176$ Flow Line

$10 / 7 \quad 440930176 \quad 23 x-176$ flow line

10/5 $4330330 \quad 176 \quad 23 x-170$ flow line

$6 / 26 \quad 2531400176 \quad 23 x-176$ flow line
$3 / 14 \quad 103104517623 x-170$ flow line

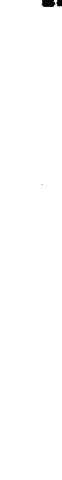

8.5065 \\ $8.5 \quad 665$}

$\begin{array}{rr}0.5 & 0 \\ 2 & 0 \\ 0.125 & 0\end{array}$

\begin{tabular}{|c|c|}
\hline 27 & 0 \\
\hline
\end{tabular}

$\begin{array}{cccccc}4 / 30 & 165 & 1100 & 285 & 248-285 \\ 12 / 24 & 567 & 1430 & 24 R & 24 R & 502 \text { Gravity Line } \\ 117 & 349 & \text { Unkn } & 24 R & 24 R \text { soz Gravity line }\end{array}$

\begin{tabular}{|c|c|c|c|}
\hline 2.5 & 0 & 140 & Internal corrosion \\
\hline 5 & 0 & 490 & Internal Corrosion \\
\hline 420 & 410 & 300 & Internal Corrosion \\
\hline \multicolumn{4}{|c|}{ 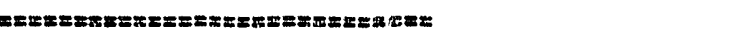 } \\
\hline 425 & 410 & 790 & \\
\hline
\end{tabular}

\begin{tabular}{|c|c|c|c|c|c|c|}
\hline$/ 19$ & 468 & 500 & is & $s$ & Gre & 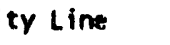 \\
\hline $3 / 12$ & 343 & 1000 & $25 s$ & 255 & Gr & ty \\
\hline $10 / 24$ & 471 & 1000 & $25 \mathrm{~s}$ & 255 & LACT & Inte \\
\hline $2 / 1$ & 528 & 050 & $5 s$ & 255 & ACT & int \\
\hline $2 / 18$ & 555 & 0800 & 255 & $25 s$ & LACT & Intake Line \\
\hline $0 / 30$ & 1485 & 1400 & 55 & $25 s$ & LACT & Intake Line \\
\hline $1 / 28$ & 420 & 45 & ss & 255 & $A C T$ & Intake \\
\hline $10 / 23$ & 468 & 1000 & $25 s$ & $25 \$$ & LACT & Intake \\
\hline $10 / 24$ & 472 & 1000 & $25 s$ & 255 & LACT & Intoke line \\
\hline 17 & 381 & 1000 & 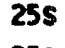 & 255 & LACT & Intoke Line \\
\hline $2 / 1$ & 537 & 0800 & $5 s$ & 58 & LACT & Intake Line \\
\hline
\end{tabular}

$\begin{array}{llllll}5 / 31 & 219 & 1000 & 24 R & 266-24 R \text { flow line } \\ 7 / 21 & 302 & 1130 & 265 & 265 \text { Gravity line } \\ 7 / 5 & 268 & 1800 & 265 & 265 \text { Gravity line } \\ 7 / 10 & 280 & 1800 & 265 & 265 \text { Gravity line } \\ 12 / 27 & 577 & 1300 & 265 & 265 \text { Gravity line } \\ & 289 & 1000 & 265 & 265 \text { Grovity line }\end{array}$



$\begin{array}{rrrl}4 & 1 & 95 & \text { Internal corrosion } \\ 8 & 0 & 140 & \text { Internal Corrosion } \\ 1 & 0 & 0 & \text { Internal corrosion } \\ 1 & 0 & 140 & \text { Internal Corrosion } \\ 3 & 2 & 95 & \text { Internal Corrosion } \\ 0.25 & 0 & 95 & \text { Internal corrosion } \\ 1 & 0 & 95 & \text { Internal Corrosion } \\ 1 & 0 & 95 & \text { Internal corrosion } \\ 2 & 0 & 50 & \text { Internal corrosion } \\ 8 & 2 & 95 & \text { Internal corrosion } \\ 5 & 2 & 95 & \text { Internal Corrosion } \\ 34.25 & 7 & 995 & \end{array}$

(1) Cleaped line

(1) Clamped line

(1) Clemped line

(1) Clamped Line

(1) Clemped Line

(1) clamed line

(i) Clamped line.

(1) Clamped line

(1) Clamped line

(1) Clemped line.

(1) Clemped Line

$\begin{array}{rrrlll}1.5 & 0 & 140 & \text { Internal Corrosion (1) Clamped leak. } \\ 1 & 0 & 80 & \text { Internal Corrosion (1) Clamped Line } \\ 1 & 0 & 0 & \text { Internal Corrosion (1) Clamped Line } \\ 3 & 0 & 90 & \text { Internal Corrosion (1) Clamped line } \\ 2 & 0 & 165 & \text { Internal Corrosion (1) Clamped Line } \\ 0.125 & 0 & 0 & \text { Internal Corrosion (1) Clamped Line }\end{array}$

\footnotetext{
- (1) CORrosion (2) Mechanical fallure (3) STUfFing boX (4) hUMAN ERror
} 
OIL SPILLS DURING 1989

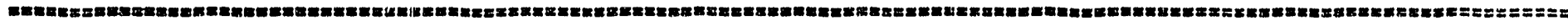
Cleanup

Rpt Estimted sarrels cost Est.

Date Mo. Time sect. Source of Spill Spllled Recovered ins Besic Ceuse * Prevent Recurrence

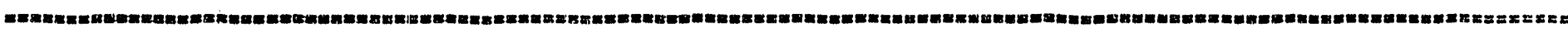

$9 / 264161200265265$ Gravity line $\quad 2 \quad 0 \quad 90$ Internal corrosion

$8 / 133452000265265$ Grovfty Line $\quad 260$ to internal corrosion

(1) clamped line.

$10 / 14221230265265$ Gravity line

$1 / 5 \quad 3 \quad 1545265265$ Gravity line

$7 / 12 \quad 2841500265265$ Grovity line

$4 / 18152 \quad 1000265265$ Gravity line

$\begin{array}{rrrl}1 & 0 & 0 & \text { Internal corrosion (1) clamped line } \\ 0.1 & 0 & 0 & \text { Internal corrosion (1) clamped line. } \\ 2 & 0 & 160 & \text { internal corrocion (1) clamped line } \\ 0.125 & 0 & 90 & \text { Internal corrosion (1) clamped line. }\end{array}$

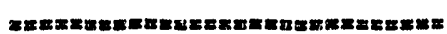

$14.35 \quad 0 \quad 745$

10/20 473 $1330 \quad 168 \quad 26-148$ rlow Line

$6 / 3 \quad 2251100285 \quad 26-28$ flow line

0.50

40

140

10/31 $490 \quad 0830 \quad 275 \quad 275$ soz Gravity line

$10 / 20 \quad 4641200 \quad 275$ 27s sOz Gravity Line

$10 / 294791030$ 27s $275 \mathrm{sOz}$ Gravity line

$10 / 22 \quad 465 \quad 1130 \quad 275 \quad 27 s$ soz ciravity Line

$10 / 16 \quad 455 \quad 1730 \quad 275 \quad 275$ s02 firevity line

$10 / 30 \quad 483 \quad 1900$ 27s 275 soz tiravity line

$10 / 30 \quad 484 \quad 1100 \quad 275275$ soz firevity Line

$0 / 28 \quad 478 \quad 1930 \quad 275275802$ tiravity Line

$10 / 19462 \quad 1100 \quad 275275802$ iravity line

$10 / 23 \quad 467 \quad 1415$ 27s 27s SOz Grevity Line

$10 / 29 \quad 6821900275275$ soz bravity Line

$10 / 14 \quad 451 \quad 1000278$ 27s-34s 502 Gravity Line

\begin{tabular}{|c|c|c|c|c|}
\hline 1 & 0.5 & 0 & Internal & corrosion \\
\hline 1 & 0 & 85 & Internal & corrosion \\
\hline 9 & 0 & 0 & Internal & corrosion \\
\hline 1 & 0 & 0 & Internal & corrosion \\
\hline 0.5 & 0 & 0 & Internal & corrosion \\
\hline 0.5 & 0 & 0 & Internal & corrosion \\
\hline 0.5 & 0 & 0 & Internal & corrosion \\
\hline 2 & 0 & 95 & Internal & corrosion \\
\hline 0.125 & 0 & 0 & Internal & corrosion \\
\hline 0.5 & 0 & 0 & Internal & corrosion \\
\hline 0.125 & 0 & 0 & Internal & corrosion \\
\hline 1 & 0 & 95 & Internal & corrosion \\
\hline \multicolumn{5}{|c|}{ 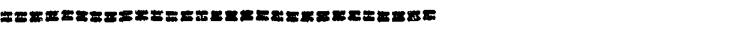 } \\
\hline 9.25 & 0.5 & $27 / 5$ & & \\
\hline
\end{tabular}

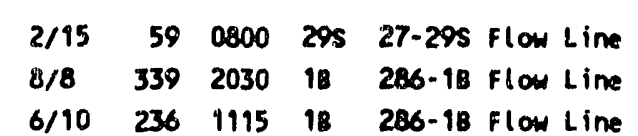

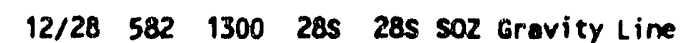

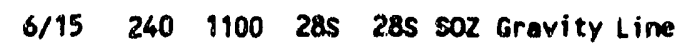

$7 / 31 \quad 328 \quad 1400 \quad 285 \quad 285 \mathrm{SOz}$ Gravity Line

$\begin{array}{llllll}7 / 12 & 282 & 1000 & 285 & 285 & 50 z \\ 9 & \text { Gravity Line }\end{array}$

$9 / 8 \quad 384 \quad 1000 \quad 285 \quad 285$ s0z Gravity line

$10 / 21 \quad 466 \quad 1400 \quad 285285502$ Gravity Line

$11 / 15 \quad 510 \quad 1330 \quad 285 \quad 285$ soz Gravity line

$12 / 15 \quad 543 \quad 0400 \quad 285 \quad 285$ soz Gravity line

$7 / 31 \quad 328 \quad 800 \quad 285 \quad 285$ s0z Gravity line

$9 / 6 \quad 383 \quad 1000 \quad 285285502$ Gravity line

$7 / 6 \quad 270 \quad 1000 \quad 285 \quad 285$ s0z Gravity Line

$8 / 13 \quad 3421000 \quad 285 \quad 285 \mathrm{s02}$ Gravity line

$7 / 12 \quad 283 \quad 1200 \quad 285 \quad 285$ soz Gravity line

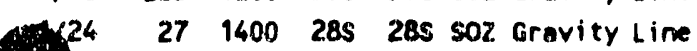

$\begin{array}{rrr}10 & 6 & 27 \\ 10 & 9 & 9 \\ 6 & 5.5 & 140 \\ 3 & 0 & 9 \\ 0.25 & 0 & 140 \\ 0.33 & 0 & 90 \\ 180 & 170 & 630 \\ 135 & 120 & 260 \\ 1 & 0 & 9 \\ 50 & 45 & 105 \\ 20 & 10 & 1250 \\ 7 & 0 & 160 \\ 0.25 & 0 & 45 \\ 0.5 & 0 & 90 \\ 0.75 & 0 & 9 \\ 35 & 30 & 31 \\ 0.125 & 0 & 90\end{array}$

Internal corrosion Internal corrosion internal corrosion

Internal corrosion Internal corrosion Internal Corrosion Internal corrosion Internal corrosion Internal corrosion Internal Corrosion Internal corrosion Internal corrosion Internal corrosion Internal corrosion Internal corrosion Internal Corrosion internal Corrosion
(1) clamped line

(1) Clamped line

(1) Clamped line

(1) clamped line

(1) clemped line

(1) Clamped line

(1) Clamped line

(1) clamped line

(i) clemped line

(1) Clamped line

(1) clamped line

(1) clamped line

(1) Clamped line

(1) Clamped line

(1) Clampod line.

(1) Replaced tee

(1) Clamped line

(1) Clamped Line

(1) Clamped line

(1) Clamped line

(1) Clemped line

(1) clamped line.

(1) Clemped line

(1) Clamped Line

(1) Clamped line

(1) Clemped line

(i) Clamped line.

(1) Clomped Line

(1) Clamped line

(1) Clamped Line

(1) Clamped line.

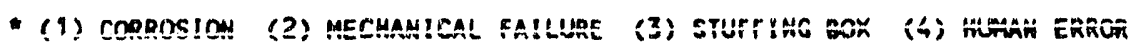


OIL SPILLS DURING 1989

cleanup

Rpt Estimed sarrele Cost Est.

Date No. Time Sect. Source of splll Spilled Recovered ins Basic couse * Prevent Recurrence

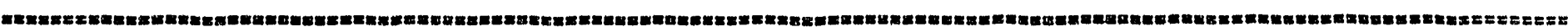

$4 / 161481200285205$ soz Gravity Line

$8 / 31371 \quad 1100$ 20s 285802 Gravity Line

$4 / 31240800285205$ soz Gravity Line

$9 / 14393$ 0730 285285 soz Gravity Líne

$1 / 24 \quad 26 \quad 1000285285502$ Gravity Líne

9/18 398 unkn 285 28S SOz Gravity Line

$9 / 7 \quad 3820900 \quad 285285502$ Gravity Line

$9 / 9387 \quad 1400 \quad 285 \quad 285$ soz Gravity line

$10 / 30 \quad 4860900285285$ s0z Gravity Line

$\begin{array}{rrrl}2 & 0 & 140 & \text { Internal corrosion } \\ 15 & 0 & 185 & \text { Internal corrosion } \\ 270 & 265 & 650 & \text { Internal corrosion } \\ 7 & 5 & 370 & \text { Internal Corrosion } \\ 0.05 & 0 & 180 & \text { internal Corrosion } \\ 4 & 0 & 95 & \text { Internal Corrosion } \\ 3 & 0 & 45 & \text { Internal Corrosion } \\ 15 & 10 & 0 & \text { Internal Corrosion } \\ 7 & 0 & 165 & \text { Internal corrosion } \\ 756.255 & 655 & 5270 & \end{array}$

(1) Clamed line.

(i) clamed line

(1) claped line.

(1) clamped line.

(1) Clamed líne.

(1) clasped line.

(1) Clamped line.

(1) Clamped line.

(1) Clamped line

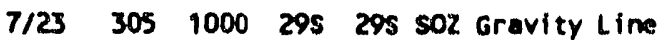

$8 / 8 \quad 340 \quad 1000 \quad 295 \quad 295$ soz Gravity line

$8 / 26 \quad 363 \quad 900295295$ s02 Gravity line

$3 / 17 \quad 107 \quad 1130 \quad 295295502$ Gravity Line

$4 / 21 \quad 1621430 \quad 295295$ soz Gravity line

ane

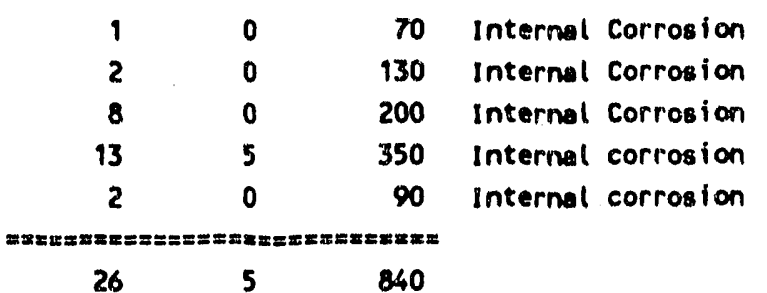

(1) clamped Line

(1) Clamped line

(1) Clamped line

(1) clamped line.

(1) clanped line.
10/26 $476 \quad 1415 \quad 116 \quad 2-116 \quad$ SOz Test Line

$10 / 27 \quad 477 \quad 1100$ 18 2-18 s0z rest Line

$11 / 54961000 \quad 30 R \quad 2-30 R$ siv shipping Line

12/19 557 unkn 30R 2-30R STV Unkn flow Line

$2 / 15 \quad 581200 \quad 33 R \quad 2-33 R$ flow line

$7 / 9 \quad 279 \quad 900 \quad 305 \quad 305$ s0z Gravity line

$3 / 27 \quad 1150900 \quad 30$ s 30 s s0z Gravity Line

$2 / 15 \quad 60 \quad 1330 \quad 26 \quad 312-26$ flow line

$3 / 11 \quad 100 \quad 1230 \quad 106 \quad 31 \times-106$ flow Line

$11 / 85090915 \quad 170 \quad 32-176$ flow Line

$7 / 6 \quad 265 \quad 1113 \quad 365 \quad 32-365$ flow line

10/31 $48906007 R$ 336-7R Flow line

$8 / 10 \quad 341930$ 33s 335 soz Grovity Line

$11 / 275190800$ 33s 335 soz Gravity Line

$12 / 9 \quad 527 \quad 1230 \quad 335 \quad 335$ SOz Grovity Line

$8 / 18 \quad 353 \quad 1600 \quad 335 \quad 33 s$ soz Gravity tine

$2 / 7 \quad 430930 \quad 335 \quad 335$ soz Gravity Line

$11 / 24930700335335$ soz Gravity line

$2 / 26 \quad 851400 \quad 335 \quad 335$ soz Gravity line

$7 / 30 \quad 324 \quad 1030 \quad 335 \quad 335$ soz Gravity line

$2 / 20 \quad 640900335335$ SOz Gravity Lime

\subsection{6 \\ Internal corrosion}

6.4

0.3

0.25

0.25

0.25

1

3

1

0.125

1

2

7

3

25

0.1

3

$$
3
$$$$
15
$$$$
50
$$

$==x= \pm x$ 185 Internal corrosion

95 Internal Corrosion

95

0

90

90

140

160

90

90
70

70

internal corrosion

internal corrosion

internal corrosion

Internal corrosion

Internal corrosion

Internal corrosion

Internal Corrosion

Internal corrosion

interrial corrosion

185

95

645

95

275

140

320

320

90

Internal Corrosion

Internal Corrosion

Internal Corrosion

Internal Corrosion

internal carrosion

Internal Corrosion

Internal corrosion

Internal Corrosion
Internal corrosion

2165
(1) Clamped line

(1) clamped line

(1) clamped line

(1) Clamped Line

(1) clamped line.

(1) Clamped line

(1) clamped line.

(1) clasped line.

(1) clamped line.

(1) Clanped Line

(1) Clamped Line

(1) clamped line

(1) Cl amped l ine

(1) Clamped Line

(1) Clamped Line

(i) clamped line

(1) Clamped line.

(1) Clamped líne

(1) Clamped line.

(1) Clamped Line

(1) Clamped line.

\footnotetext{
- (1) corrosion (2) Mechanical failure (3) Stuffing gox (4) mLMan ERror
} 
DIL SPILLS OURING 1909

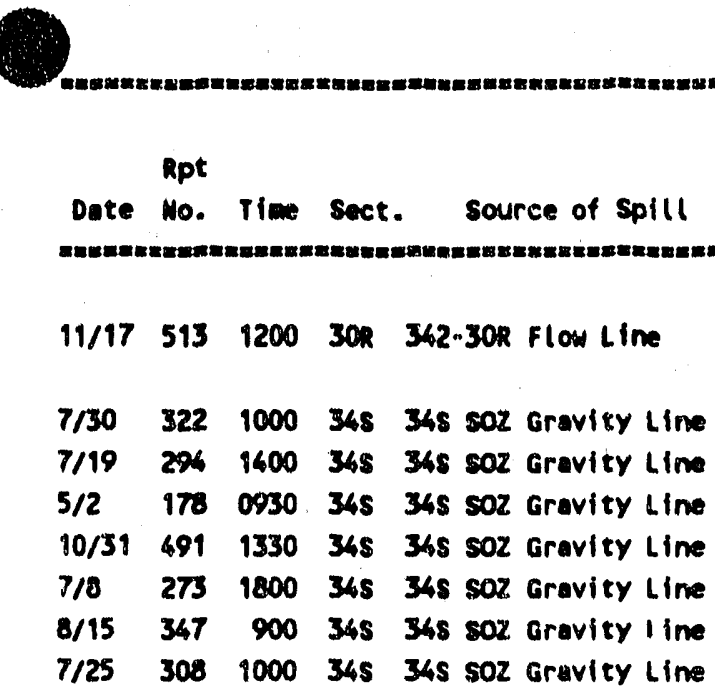

\begin{tabular}{|c|c|c|c|c|}
\hline $11 / 6$ & 500 & 1430 & 295 & 34-29s Flow line \\
\hline $9 / 19$ & 397 & 0100 & $35 R$ & 35R STV Gravity line \\
\hline $12 / 5$ & 530 & 0500 & $35 s$ & 35s Grovity Line \\
\hline $2 / 26$ & 80 & 1000 & 16 & 35-16 Gas line \\
\hline $4 / 14$ & 144 & 1700 & 36 & 362-36 Flow line \\
\hline $3 / 19$ & 104 & 0900 & $\pi$ & 362-7R Flost Line \\
\hline & 379 & 1220 & $26 n$ & 363-261 Flowl ine \\
\hline & $\infty$ & 0900 & 100 & 36-36 Flow Line \\
\hline $2 / 28$ & $\mathbf{2 3}$ & 1200 & 106 & 36.36 flow tine \\
\hline $6 / 11$ & 236 & 1000 & 36 & $371-36$ flow line \\
\hline $1 / 30$ & 35 & 0700 & 345 & 371-36 flow line \\
\hline $1 / 31$ & 31 & 0930 & $34 S$ & 371.36 flow Line \\
\hline $8 / 11$ & 344 & 1830 & 36 & 371-36 flow Line \\
\hline $1 / 13$ & 12 & 1700 & 36 & 371-36 flow line \\
\hline
\end{tabular}

Eetimated Barrels Cost Eat. spllled Recovered in 5
Action to

Basic Couse
Prevent Recurrence

\begin{tabular}{|c|c|c|c|c|c|c|}
\hline 53.6 & 50 & 185 & Internal & Corrosion & (1) & Replaced Union \\
\hline 1 & 0 & 0 & Internal & corrosion & (1) & clasped Line \\
\hline 1 & 0 & 0 & Internal & corrosion & (1) & Clauped LIne \\
\hline 0.5 & 0 & $\mathbf{0}$ & Internal & Corrosion & (1) & Clamped leak. \\
\hline 0.125 & $\mathbf{0}$ & 0 & Internal & corrosion & (1) & Clamed line \\
\hline 1 & 0 & 90 & Internal & corrosion & (1) & clamped line \\
\hline 3 & 0 & 0 & Internal & Corrosion & (1) & Clemped line \\
\hline 2 & 0 & 0 & Internal & Corrosion & (1) & Cleaped line \\
\hline \multicolumn{7}{|c|}{ 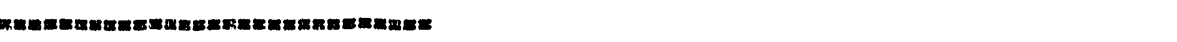 } \\
\hline 8.625 & 0 & 90 & & & & \\
\hline 1 & 0.5 & 165 & Internal & Corrosion & (1) & Clemped Line \\
\hline 5 & 0 & 95 & Internal & Corrosion & (1) & Clamped line. \\
\hline 2 & 0 & 70 & Internal & Corrosion & (1) & Clamped line \\
\hline 0.125 & 0 & 45 & Internal & corrosion & (1) & clemped line. \\
\hline 0.5 & 0 & 45 & Internal & corrosion & (1) & Clesped line. \\
\hline 10 & 0 & 400 & Internal & corrosion & (1) & Clamped line. \\
\hline 1 & 0 & 230 & Intornal & Corrosion & (1) & clamped line. \\
\hline 2 & 0 & 0 & Internal & corrosion & (1) & clamped line. \\
\hline 2 & 0 & 90 & Internal & corrosion & (1) & Clmpod line. \\
\hline 1 & 0 & 90 & Internal & corrosion & (1) & Clemped line \\
\hline 0.125 & 0 & 45 & Internal & Corrosion & (1) & Clamped line. \\
\hline 0.125 & 0 & 45 & Internal & Corrosion & (1) & Claxped line. \\
\hline 1 & 0 & 165 & Internal & Corrosion & (1) & Replaced sec. of line \\
\hline 0.06 & 0 & 45 & Internal & corrosion & (1) & clemped line. \\
\hline \multicolumn{7}{|c|}{ 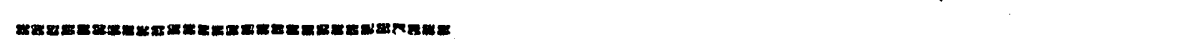 } \\
\hline 2.31 & 0 & 390 & & & & \\
\hline 2 & 9 & 40 & Internal & Corrosion & (1) & clapped line. \\
\hline 3 & 0 & 140 & Internal & Corrosion & (1) & clamped line. \\
\hline 0.1 & 0 & 80 & Internal & Corrosion & (1) & Claxped line \\
\hline 2 & 0 & 90 & Internal & corrosion & (1) & Clamped line. \\
\hline 5 & 0 & 985 & Internal & corrosion & (1) & Clemped line \\
\hline 0.5 & 0 & 45 & Internal & Corrosion & (1) & clamped line. \\
\hline 5 & 4 & 0 & Internal & Corrosion & (1) & Clamped line. \\
\hline 0.75 & 0 & 45 & Valve cor & rrosion & (9) & Repalced valve. \\
\hline 0.125 & 0 & 50 & Internal & corrosion & (1) & Replaced line \\
\hline 1 & 0 & 180 & Internal & Corrosion & (1) & Repu ired Tank \\
\hline 5 & 0 & 185 & Internol & Corrosion & (1) & clasped line \\
\hline 2 & 0 & 45 & Internal & corrosion & (1) & clamped line. \\
\hline 0.1 & 0 & 200 & Internal & Corrosion & (1) & Clamped Line \\
\hline
\end{tabular}

\begin{tabular}{|c|c|c|c|c|}
\hline $9 / 5$ & 378 & 1500 & $35 R$ & 373-35R flowline \\
\hline $1 / 22$ & 21 & 1700 & 36 & 373-36 Flow line \\
\hline $7 / 26$ & 318 & 2000 & $28 R$ & 378-28R flow line \\
\hline $4 / 12$ & 139 & 0100 & 86 & 37.86 flow line \\
\hline $10 / 15$ & 452 & 1100 & 26 & 38-26 Flow line \\
\hline $1 / 12$ & 9 & 1130 & 10 & 3-16 502 Gravity Line \\
\hline $9 / 29$ & 421 & 1400 & $27 s$ & 3-27s vR Gravity line \\
\hline $4 / 13$ & 169 & 0830 & $28 s$ & 3-285 s0z Separator \\
\hline $10 / 6$ & 443 & 1030 & 295 & 3-295 S0Z Grevity Line \\
\hline $7 / 27$ & 315 & 1000 & $30 s$ & 3-30S STV Stock Tank \\
\hline $8 / 26$ & 362 & 1000 & 345 & 3-34S STV Discharge Line \\
\hline $2 / 24$ & $\pi$ & 0800 & 365 & 3-36s soz Intake line \\
\hline $11 / 28$ & 521 & 1130 & 66 & 3-6G soz Injection line \\
\hline
\end{tabular}

men 
OIL SPILLS OURIMG 1989

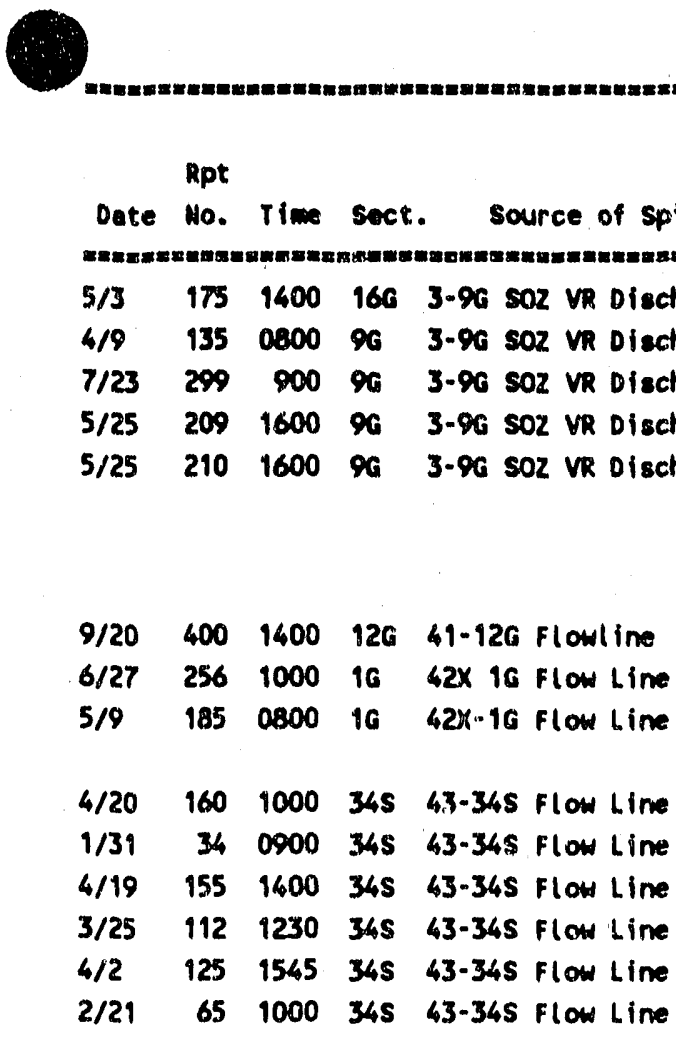

4

$5 / 19$

207 Unkn 86

$\begin{array}{llll}4 / 17 & 151 \quad 1400 \quad 46\end{array}$

$11 / 13 \quad 508 \quad 2400 \quad 16$

$\begin{array}{llll}10 / 5 \quad 434 & 1100 \quad 10\end{array}$

$4 / 1 \quad 123 \quad 0530 \quad 148$

$\begin{array}{llll}1 / 28 & 33 & 1030 & 186\end{array}$

$8 / 27$

$7 / 13286900295$ 4-295 Soz Chemical Line

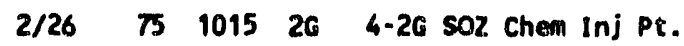

$3 / 26 \quad 113 \quad 1130 \quad 305 \quad 4.305$ SOz Gravity line

$10 / 9 \quad 446 \quad 1200 \quad 335 \quad 4-335$ SOz Gravity line

4/12 $140 \quad 1745 \quad 56 \quad 4-56 \quad 502$ Gravity line

4/15 $146 \quad 1715 \quad 56 \quad 4-56$ s0z Grovity Line

4/11 138 unkn 50 4-56 s0z Gravity Line

$3 / 14 \quad 102 \quad 0800 \quad 56 \quad 4-56502$ Gravity Line

$4 / 3 \quad 126 \quad 0400 \quad 56 \quad 4-56 \quad 50 z$ Gravity line cleonup

Eatinated Barrels Cost Est. Spilled Recovered in

in $\$$ Becic Cause

Action to

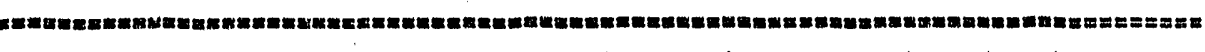

$\begin{array}{rrrrl}52 & 48 & 190 & \text { Internal Corrosion } \\ 0.5 & 0 & 0 & \text { Internal corrosion } \\ & 9 & 8 & 160 & \text { Internal Corrosion } \\ 4 & 0 & 200 & \text { Internal Corrosion } \\ & 3 & 0 & 0 & \text { Internal Corrosion } \\ & 40.5 & 56 & 550 & \end{array}$

(1) Clamped leak.

(1) claxped line.

(1) Clamped Line

(1) clamped leak.

(1) Clamped leak.

$\begin{array}{rrrl}2.5 & 0 & 140 & \text { Internal corrosion } \\ 5 & 2 & 160 & \text { Internal corrosion } \\ 9 & 5 & 320 & \text { Internal corrosion } \\ 0.125 & 0 & 0 & \text { Internal corrosion } \\ 0.125 & 0 & 90 & \text { Internal Corrosion } \\ 0.125 & 0 & 140 & \text { Internal corrosion } \\ 1 & 0 & 45 & \text { Internal corrosion } \\ 2 & 0 & 200 & \text { Internal corrosion } \\ 0.25 & 0 & 160 & \text { Internal corrosion } \\ 3.625 & 0 & 635 & \end{array}$

Internal Corrosion Internal corrosion Internal Corrosion Internal corrosion Internal corrosion Internat Corrosion Internal Corrosion Internal Corrosion Internal corrosion internal corrosion Internal corrosion

Internel corrosion Internat corrosion Internal corrosion Internal corrosion Internal corrosion
(1) clamped line.

(1) clamped line

(1) clamped leak.

(1) Clamped lirve.

(1) clamped line.

(1) clamped line.

(1) Clamped line.

(1) clamped line.

(1) clamped line.
(1) Clamped leak.

(1) clamped line.

(1) Replaced Line

(1) Replaced line

(1) clamped line.

(1) Clumped line.

(1) Replaced collar

(1) Replaced fitting

(1) Replaced nipple.

(1) clamped line.

(1) clamped line

(1) Clamped line.

(1) clanped line.

(1) clamped line.

(1) clamped line.

(1) clamped line.

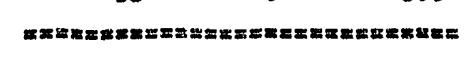

$\begin{array}{lll}51.5 & 0 & 1310\end{array}$

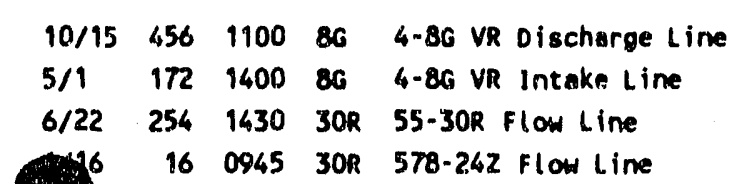

$\begin{array}{rrr}1 & 0 & 95 \\ 3 & 0 & 90 \\ 1 & 0 & 230 \\ 1 & 0 & 42\end{array}$

Internal corrosion Internal Corrosion Internal corrosion Internal Corrosion
(1) Clamped line

(1) clamped leak.

(1) Clamped line

(1) Clamped line.

\footnotetext{
- (1) corrosion (2) mechanical failure (3) stuffing box (4) hlman error
} 
OIL SPILLS DURING 1989

\begin{tabular}{|c|c|c|c|c|c|c|c|c|c|c|c|}
\hline \multirow{2}{*}{$\begin{array}{l}\text { Date } \\
\text { =ammant }\end{array}$} & \multirow{2}{*}{$\begin{array}{l}\text { Rpt } \\
\text { No. }\end{array}$} & \multirow[t]{2}{*}{ Time } & \multirow[t]{2}{*}{ soct. } & \multirow[t]{2}{*}{ Source of spill } & \multicolumn{2}{|c|}{$\begin{array}{l}\text { Estimated Barrels } \\
\text { spllled Recovered }\end{array}$} & \multirow[t]{2}{*}{$\begin{array}{l}\text { Cleenup } \\
\text { Cost Est. } \\
\text { in } \$\end{array}$} & \multirow[t]{2}{*}{ Desic C } & & \multirow{2}{*}{\multicolumn{2}{|c|}{$\begin{array}{l}\text { Action to } \\
\text { Prevent Recurrence }\end{array}$}} \\
\hline & & & & & & & & & & & \\
\hline $6 / 1$ & 220 & Unkn & 295 & 57-29s flow hine & 1 & 0 & 90 & Internal & corrosion & (1) & Clemped line \\
\hline $8 / 3$ & 331 & 800 & 295 & 57-29s Flow Line & 1.25 & 0 & 90 & Internal & Corrosion & (1) & Clemped line \\
\hline $5 / 14$ & 190 & 0900 & 285 & 58x-285 flow line & 1 & 0 & 90 & Internal & Corrosion & (1) & Clemped leak. \\
\hline $6 / 22$ & 249 & Unkn & 285 & 58x-28s flow Line & 0.25 & 0 & 45 & Internal & corrosion & (1) & Clamped line \\
\hline $5 / 23$ & 204 & 1145 & 10 & 58-16 flow line & 2 & 0 & 90 & Internal & Corrosion & (1) & Clemped leak. \\
\hline $5 / 22$ & 208 & 0900 & 36 & 58-36 flow Line & 5 & 0 & 180 & Internal & Corrosion & (1) & Clemped leak. \\
\hline $8 / 28$ & 365 & 1130 & 176 & 61-176 Flow Line & 2 & 2 & 140 & Internal & Corrosion & (1) & clamped line \\
\hline $9 / 2$ & 375 & 1100 & 18 & 65-18 flowline & 3 & 0 & 140 & Internal & Corrosion & (1) & clamped line. \\
\hline $12 / 15$ & 542 & 0400 & 285 & 66-28s Flow Line & 20 & 10 & 705 & Internal & Corrosion & (1) & Clemped line \\
\hline $3 / 6$ & 93 & 0845 & 96 & 67.96 flow line & 3 & 0 & 90 & Internal & corrosion & (1) & Clemped line. \\
\hline $5 / 19$ & 198 & 0900 & 285 & $6^{N} \mathrm{SOz}$ Gravity Line & 0.125 & 0 & 90 & Internal & Corrosion & (1) & Clemped leok. \\
\hline $6 / 25$ & 252 & 100 & 46 & $72-46$ flow line & 3 & 0 & 35 & Internal & corrosion & (1) & Clamped line \\
\hline $10 / 23$ & 470 & 0845 & 56 & T5-50 flow line & 0.5 & $\mathbf{0}$ & 0 & Internal & corrosion & (1) & Clamped line \\
\hline $6 / 3$ & 221 & 1045 & 96 & 76.90 Flow Line & 5 & 0 & 180 & Internal & corrosion & (1) & Clemped line \\
\hline $8 / 17$ & 350 & 1030 & 26 & 78-20 flow line & 1 & 0 & 70 & Internal & Corrosion & (1) & Clamped line \\
\hline $1 / 1$ & 1 & 0700 & 96 & 81ME-96 flow line & 0.25 & 0 & 90 & Internal & Corrosion & (1) & Clemped line. \\
\hline $9 / 3$ & 374 & 1300 & 56 & 87-56 flowline & 0.25 & 0 & 0 & Internal & Corrosion & (1) & clamped line. \\
\hline $10 / 19$ & 461 & 0930 & 86 & 86 soz Grovity Line & 25 & 25 & 95 & Internal & corrosion & (1) & Clamped line \\
\hline $10 / 28$ & 480 & 0900 & 86 & $86 \mathrm{SOz}$ Gravity Line & 7 & 2 & 245 & Internal & corrosion & (1) & Clamped line \\
\hline & 369 & 930 & 86 & 86 SOz Grevity Line & 6 & 3 & 90 & Internal & Corrosion & (1) & Clempad line \\
\hline & 32.5 & 1800 & 46 & Flow Line & 1 & 0 & 40 & internal & corrosion & (1) & clamped Line \\
\hline $6 / 5$ & 230 & 1930 & $23 s$ & Morth Flenk Gravity Line & 2 & 0 & $\mathbf{0}$ & Internal & corrosion & (1) & Clamped line \\
\hline $5 / 5$ & 180 & 1730 & $23 s$ & North Flank Grovity Line & 1 & 0 & 90 & Internal & Corrosion & (1) & clemped leak. \\
\hline $6 / 1$ & 215 & 2230 & 235 & North Flank Gravity Line & 2 & 0 & 0 & Internal & corrosion & (1) & Clamped line \\
\hline $4 / 4$ & 131 & 1230 & $23 s$ & North Flank Gravity Line & 0.5 & 0 & 90 & Internal & corrosion & (1) & Clamped line. \\
\hline $6 / 2$ & 226 & 2030 & $23 s$ & North Flank Gravity Line & 1 & 0 & 90 & Internal & corrosion & (9) & Clamped line \\
\hline $3 / 23$ & 190 & 1600 & 235 & Morth Flank Gravity Line & 8 & 6 & 200 & Internal & corrosion & (1) & clamped line. \\
\hline $5 / 22$ & 203 & 1500 & $23 s$ & Morth Flank Gravity Line & 31 & 30 & 200 & Internal & Corrosion & (1) & Clamped leak. \\
\hline $9 / 11$ & 388 & 1900 & $23 s$ & Morth Flonk Gravity Line & 0.5 & 0 & 0 & Internol & Corrosion & (1) & Clamped line. \\
\hline $4 / 27$ & 164 & 0800 & 235 & North Flank Gravity Line & 8 & 7 & 90 & Internal & corrosion & (1) & Clamped line. \\
\hline $91 / 29$ & 52.6 & 1115 & $25 s$ & Morth Flonk Gravity Line & 8 & 0 & 140 & Internal & Corrosion & (1) & Clamped line \\
\hline $1 / 9$ & 6 & 1145 & 235 & Morth Flank Gravity Line & 0.125 & 0 & 90 & Internal & Corrosion & (g) & clamped line. \\
\hline $3 / 9$ & 97 & 1700 & $23 s$ & Morth Flank Gravity Line & 0.5 & 0 & 90 & Internal & corrosion & (1) & Clamped line. \\
\hline $6 / 16$ & 242 & 1400 & 235 & North Flank Grovity Line & 9 & 0 & 90 & Internal & corrosion & (9) & Clamped line \\
\hline $6 / 27$ & 257 & 1445 & 235 & North Flank Gravity Line & 1 & 0 & 90 & Internal & corrosion & (1) & Clemped line \\
\hline $2 / 12$ & 49 & 1800 & $23 s$ & Morth Flank Gravity Line & 2 & 1 & 90 & Internal & corrosion & (1) & Clamped line. \\
\hline $7 / 9$ & 275 & 1300 & $23 s$ & North Flank Gravity Line & 2 & 1 & 90 & Internal & Corrosion & (1) & Clamped Line \\
\hline $6 / 16$ & 241 & 1400 & $23 s$ & Morth Flank Gravity Line & 1 & 0 & 45 & Internol & corrosion & (1) & Clamped line \\
\hline $9 / 9$ & 385 & 0800 & $25 s$ & Morth Flonk Grovity Line & 2 & 0 & 70 & Internal & Corrosion & (1) & Clmped line. \\
\hline $6 / 4$ & 228 & 1200 & $23 s$ & North Flonk Grovity Line & 2 & 0 & 0 & Internal & corrosion & (1) & Clamped line \\
\hline $7 / 5$ & 267 & 1800 & $23 s$ & Morth Flank Gravity Line & 2 & 1 & 100 & Internal & Corrosion & (1) & Clamped Line \\
\hline $7 / 6$ & 271 & 800 & $23 s$ & North Flank Grovity Line & 3 & 0 & 45 & Internal & Corrosion & (1) & Clamped Line \\
\hline $6 / 11$ & 233 & 600 & $23 s$ & North Flank Gravity Line & 3 & 1 & 90 & Internal & corrosion & (1) & Clamped line \\
\hline 428 & 259 & 1300 & $23 s$ & North Flonk Gravity Line & 0.5 & 0 & 90 & Internal & corrosion & (1) & Clamped line \\
\hline
\end{tabular}

- (1) corrosion (2) Mechanical failure (3) Stuffing gox (4) hLMan ERror 
OIL. SPILLS DURING 1989

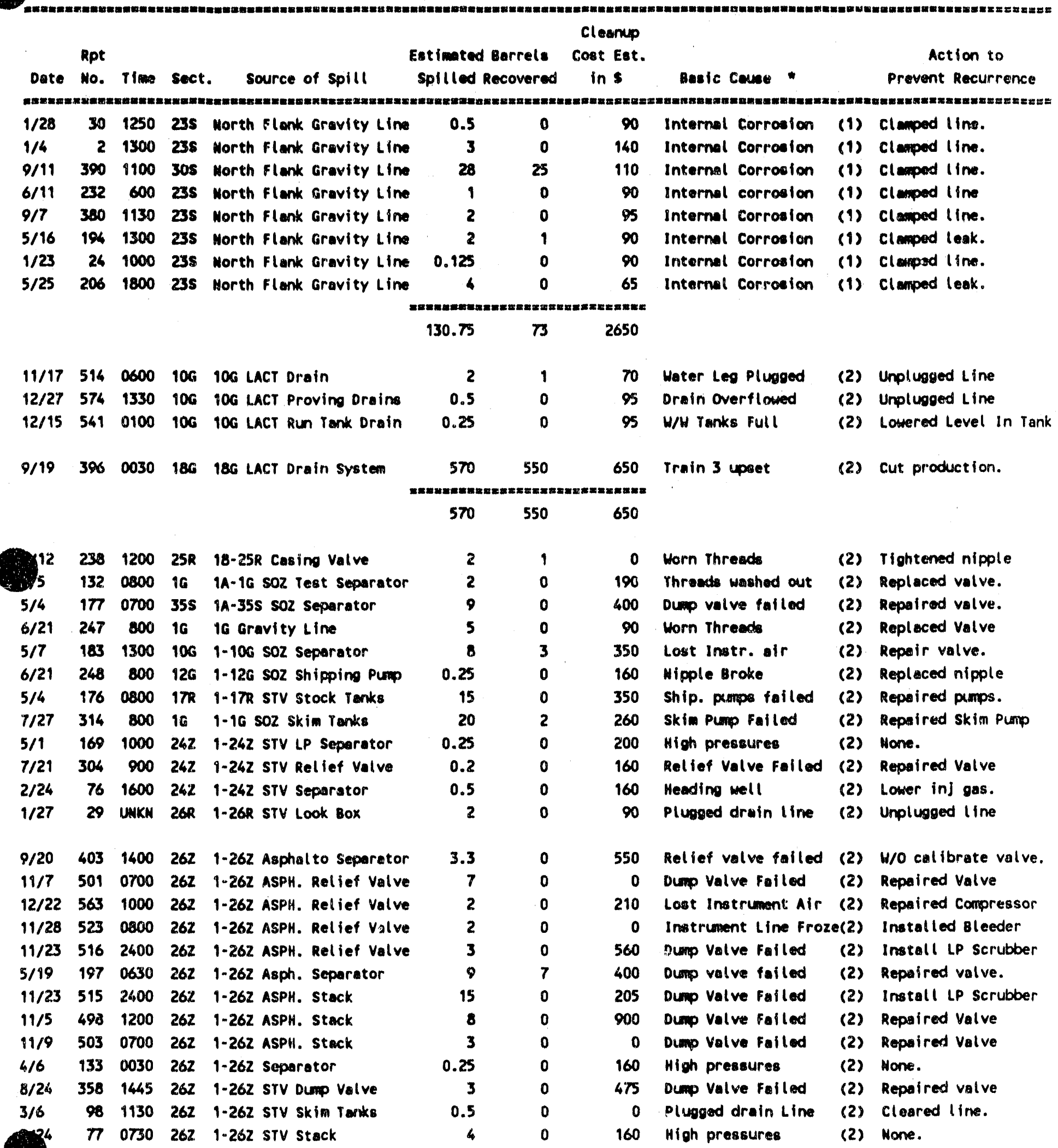

\footnotetext{
- (1) CORrosion (2) MECHAHICAL fAILURE (3) STUfFing bOX (4) MLMAH ERror
} 
OIL SPILLS OURIMG 1989

\section{Cleenup}

Rapt Estinated surrels Coot Est.

Date Mo. Tim sect. Source of solll sollled Rerovered

Dasic Cause *

Action to $10 / 27$ 491 Unkn 262 1-262 sTV secek

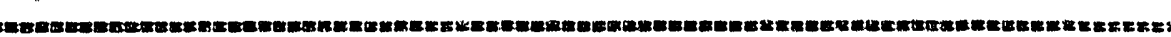

10/11 447 1430 29R 9-2\%R STV skim Tanks

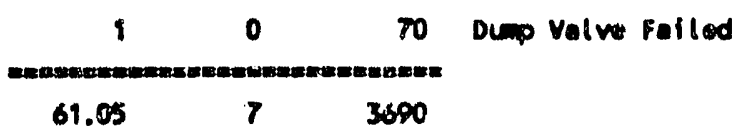

(2) Repoired valve

$5 / 14$ 191 0030 zQk 1-2\%R sTV skin TK

$\begin{array}{rlll}1.3 & 0 & 190 & \text { Pup failed } \\ 0.5 & 0 & 260 & \text { Pupe failled } \\ 18 & 0 & 165 & \text { Pup foiled } \\ 0.33 & 0 & 165 & \text { Flenge Loaked }\end{array}$

(2) Repaired purp

$12 / 275810230324$ 1-32s stV Drain Tank

$10 / 84420500325$ 1-32s sTV shipping pups

0.33

$9 / 20401 \quad 1200 \quad 332 \quad 1-332$ sTV sepereter

$11 / 10$ sok unikn 33R 9-33R sTV shippiro pup

$12 / 175640900$ 33R 1-33R sTV steck

$5 / 124871200$ 33R 1-33R STV stack

$10 / 14 \quad 469103033 R \quad 1-33 R$ STV stack

$5 / 5181 \quad 1500 \quad 33 R \quad 1-33 R$ sTV stack

$7 / 23 \quad 301 \quad 164533 R \quad 1.33 R$ sTV stack

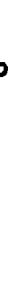

2
2
1
1.5
1
1
1.5

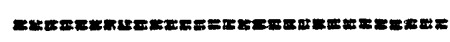

10

$0 \quad 105$

10S High preseustes

Horn Paeking

Compresuors Doun

High preseures

165 stack Valve failed

(2) Repaired pumps.

(2) Repaired Pump

(2) Replaced gasket



(29 3201201360 1-34h STV Rel ief Valwe

$4 / 11536030034 R$ 1-34R sTV Relfef valve

$4 / 16145173034 k 1-34 R$ sTV skin Tenks.

$\begin{array}{rrrl}0.5 & 0 & 80 & \text { Relief valve falled } \\ 0.1 & 0 & 75 & \text { Dup balve failed } \\ 15 & 0 & 750 & \text { Pup follod }\end{array}$

(2) None.

(2) Repleced Pocking

(2) None

(2) Increased chen.

(2) Morre

(2) Check relief valve.

260 High pressures

Dum Valve Foiled

(2) Replaced Valve
(2) Repoired Valve

(2) Replaced Vaive

(2) Repaired pump.

(2) Repaired Volve

(2) Repoired Pump

(2) Repaired valve.

(2) Drain sep. slower

(2) Recet air comp.
$7 / 72724500$ 34s 1-34s Relief valve

$7 / 8 \quad 274$ 1100 345 1-345 skim Tenks

$3 / 30 \quad 198 \quad 2400 \quad 345 \quad 1-345502 \mathrm{BPQ}$

$12 / 30 \quad 583 \quad 1000 \quad 365 \quad 9-3 / 4502$ arain $\operatorname{lax}$

$3 / 24111 \quad 1300 \quad 345 \quad 1-345$ s02 separtor

$219 \quad 47 \quad 1800 \quad 365 \quad 1-365$ saz Druin bax

$2 / 10 \quad 51 \quad 1930 \quad 46 \quad 1.46$ stV skim Tks

3
2
1
0.125
5

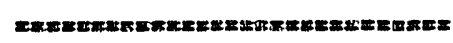

$11.125 \quad 0 \quad 885$

0.125

0

4. Power failure

(2) Brpassed setting.

990

$=2 \pi x=1 \times$

930

4500 Main switch falled

(2) Repaired awitch.

$990 \quad 930 \quad 4500$

$\begin{array}{lrlll}1 / 6 & 5 & 0200 & 56 & 1-56 \text { sTV skim Tk } \\ 10 / 30 & 488 & 0730 & 7 R & 1-7 R \text { sTV shipping Pump } \\ 1 / 19 & 18 & 1000 & 7 R & 1-7 R \text { sTV stack } \\ 2 / 14 & 56 & 1700 & 7 R & 1-7 R \text { sTV stack } \\ 9 / 28 & 499 & 1500 & 345 & 21-34 \text { s cellur orain } \\ 7 / 12 & 288 & \text { unkn } & 18 & 22-18 \text { stuffing Box } \\ 2 / 63 & 6 & 1800 & 285 & 285 \text { condensate th }\end{array}$

$\begin{array}{rr}25 & 19 \\ 1 & 0 \\ 7 & 0 \\ 20 & 10 \\ 3 & 0 \\ 0.25 & 1 \\ 2 & 0\end{array}$

ro0 Power failure
165 Gewoe Dikessembled
160 Stg $\$ / 1$-no olarm
350 PRV failed
160 Drain ran over
90 Worn Packing
160 Liquid in lines

(2) Non

(2) Replaced souse

(2) Checked alorm

(2) Repoired valve.

(2) Plus drain line.

(2) Replaced Pocking

(2) Pulled gos lines.

\footnotetext{
- (1) corrosion (2) mechamical fallure (3) stuffimg gox (4) muan error
} 
OIL. SPILLS DURIMG 1909

cleonup

Rnt

Date Mo. Tine sect. Source of spill

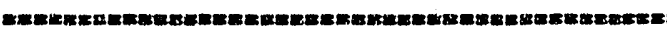

$10 / 84380800$ 2SR 2-25R STV LP Separator

$8 / 22361$ Unkn 25R 2-25R STV Rellef Valve

$12 / 26579100025 R \quad 2-25 R$ STV Relfef Votve

$7 / 2531050025 R \quad 2-25 R$ sTV Relfef Valve

$7 / 102051200$ 25R 2-25R sTV Relief Valve

$7 / 19295100025 R \quad 2-25 R$ sTV Relief Valve

6/11 $23790025 R$ 2-25R STV Relief Valve

9/11 3860800 25R 2-25R STV separator

$5 / 131880930 \quad 25 R \quad 2-25 R$ STV Separator

$10 / 8 \quad 6400000$ 25R 2-25R STV Skim Tenk
Estimated sarrels Cost Eet.

spilled Recovered in $s$
Action to

Prevent Recurrence

\begin{tabular}{|c|c|c|c|c|c|c|}
\hline 1 & 0 & 165 & Rellef Volve & Folled & (2) & Mone \\
\hline 0.1 & 0 & 165 & Rellef valve & Felled & (2) & Repufred volve \\
\hline 2 & 0 & 165 & Rellef Volvo & Folled & (2) & Adjued Stack Valve \\
\hline 1 & 0 & 290 & Relief Velve & Falled & (2) & Repeired Velve \\
\hline 0.125 & 0 & 240 & Rellef Valve & Falled & (2) & Repaired Valve \\
\hline 0.125 & 0 & 160 & Rellef Volve & Falled & (2) & Replaced Velve \\
\hline 0.5 & 0 & 160 & Rellef valve & Folled & (2) & Adjusted stack valve \\
\hline 0.1 & $\mathbf{0}$ & 170 & Heading well & & (2) & Adjust BPR. \\
\hline 1 & $\mathbf{0}$ & 350 & Heading mell & & (2) & None. \\
\hline 1 & 0 & 210 & Punp Failed & & (2) & Repaired pump \\
\hline
\end{tabular}

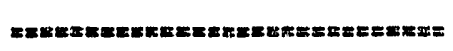

6.9502075

$\begin{array}{lrllll}12 / 10 & 535 & 1500 & 27 R & 2-27 R \text { sTV Relief Valve } \\ 4 / 13 & 142 & 1600 & 27 R & 2-27 R \text { sTV Relief Valve } \\ 2 / 23 & 68 & 0800 & 27 R & 2-27 R \text { sTV stack } \\ 1 / 20 & 19 & 0700 & 27 R & 2-27 R \text { sTV stack } \\ 9 / 25 & 413 & 1300 & 27 R & 2-27 R \text { sTV stack } \\ 7 / 28 & 316 & 1130 & 27 R & 2-27 R \text { sTV stack } \\ 91 & 370 & 1030 & 27 R & 2-27 R \text { sTV stack } \\ 9 & 7 & 1145 & 27 R & 2-27 R \text { sTV stock }\end{array}$

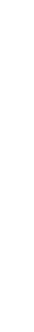

$\begin{array}{rrr}0.5 & 0 & 75 \\ 0.5 & 0 & 250 \\ 0.25 & 0 & 160 \\ 2 & 0 & 200 \\ 0.5 & 0 & 75 \\ 1 & 0 & 70 \\ 0.5 & 0 & 70 \\ 5 & 0 & 200\end{array}$

Relief Valve Failed (2) Recalibroted Valve Dusp valve plupged (2) Repaired valve. MP chup valve folled (2) Repaired valve. HI-Ivl switch falled (2) kepaired suitch Plusoed controller (2) Repaired corvtroller. Controller Failed (2) Repaired Controller Air Compressor Foiled(2) Repaired Compressor ESD failed (2) Repaired ESD

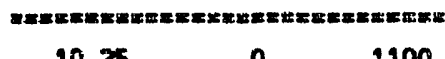

10.2501100

$7 / 283192130292$ 2-29R sTV Relief Valve 0.10 $11 / 27520 \quad 110029 R \quad 2-29 R$ STV Skim Tanks

0.10

160

Relief Valve foiled

(2) Repaired Valve

70

Punp Failed

(2) Repaired Pump

4/6 $134 \quad 1300 \quad 300 \quad 2-30$ cern Relief valve

4/2 $122080030 R \quad 2-30 R$ Carn Skim Tenk

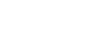

3/31 121 0800 300 2-300 Carn skim Tk

$5 / 51221500 \quad 300 \quad 2-300$ carn stack

$4 / 30167 \quad 1100 \quad 302 \quad 2-302$ carn stack

$5 / 2 \quad 1730530$ 30R 2-30\% carn stack

$7 / 31326 \quad 90030 \mathrm{R}$ 2-30R CARH stock

$8 / 7338113030 R$ 2-30R CARH. Drain Tonk

$8 / 2335780030 \mathrm{R}$ 2-30R CARN. Relief Volve

$6 / 923120030 R$ 2-30R CARM. Stack

$\begin{array}{rr}1 & 0 \\ 50 & 48 \\ 60 & 58 \\ 0.125 & 0 \\ 0.5 & 0 \\ 0.5 & 0 \\ 0.3 & 0 \\ 0.25 & 0 \\ 0.1 & 0 \\ 2 & 0\end{array}$

500

230

6.8

0

300

250

160

85

130

160

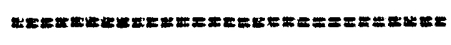

$114.775 \quad 106 \quad 1883$

7/26 311 $800 \quad 300 \quad$ 2-30R Shipping Pum

$12 / 22570100030 R \quad 2-30 R$ STV Relief Valve

$\begin{array}{ll}0.5 & 0 \\ 0.5 & 0 \\ 1.7 & 0 \\ 0.2 & 0 \\ 0.5 & 0\end{array}$

$8 / 5 \quad 336 \quad 745 \quad 30 R \quad 2-30 R$ sTV Relief Valve

$9 / 453941000300 \quad 2-302$ sTV separator

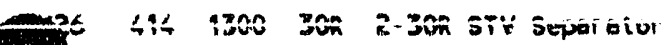

พ.ร

90
130
750
165

ט
High pressures

Level switch failed

Hi IVI switch failed

High pressures

Wigh pressures

(2) Mone.

(2) Repoir switch.

(2) Reploced switch.

High pressures

(2) None.

Stock Valve failed

(2) SSV's in service.

Drain Pump Failed

(2) None.

Relief Volve failed

(2) None

(2) Repaired purnp

Relief Valve failed

(2) Repaired valve

(2) Hone

Horn Pucking

(2) Repleced Pecking

Relief Valve failed

(2) Repoired Valve

Relief Valve Failed

(2) Repaired Valve

Compressors failed

(2) None.

inign pressure

(2) Rone.

- (1) corrosion (2) mechanical failure (3) stuffimg box (4i) hlmam error 


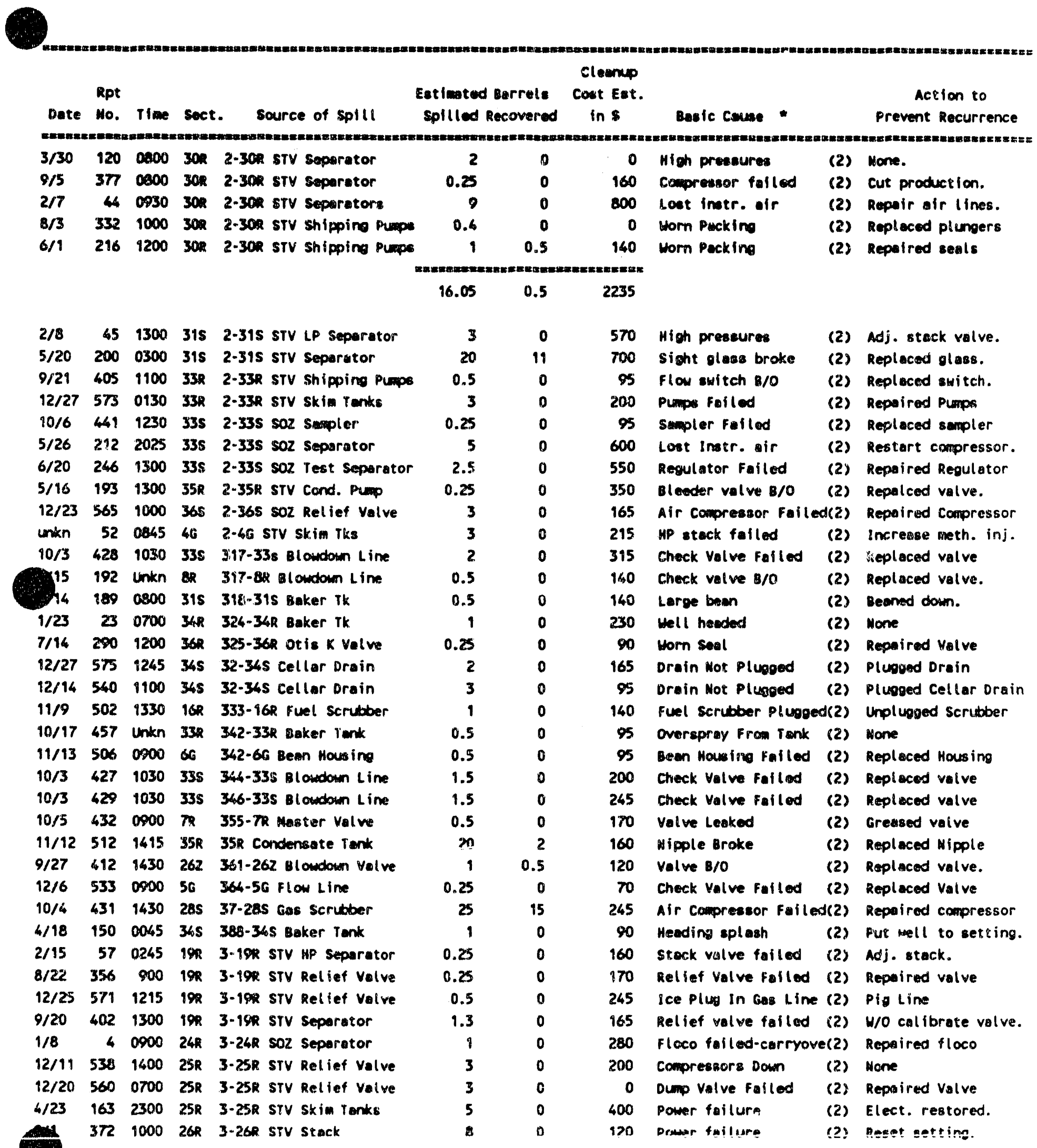

\footnotetext{
- (1) corkosion (2) MechaMical. FAILURE (3) STUFFIHG doX (4) MUMAN ERror
} 
OIL SPILLS DURIHG 1989

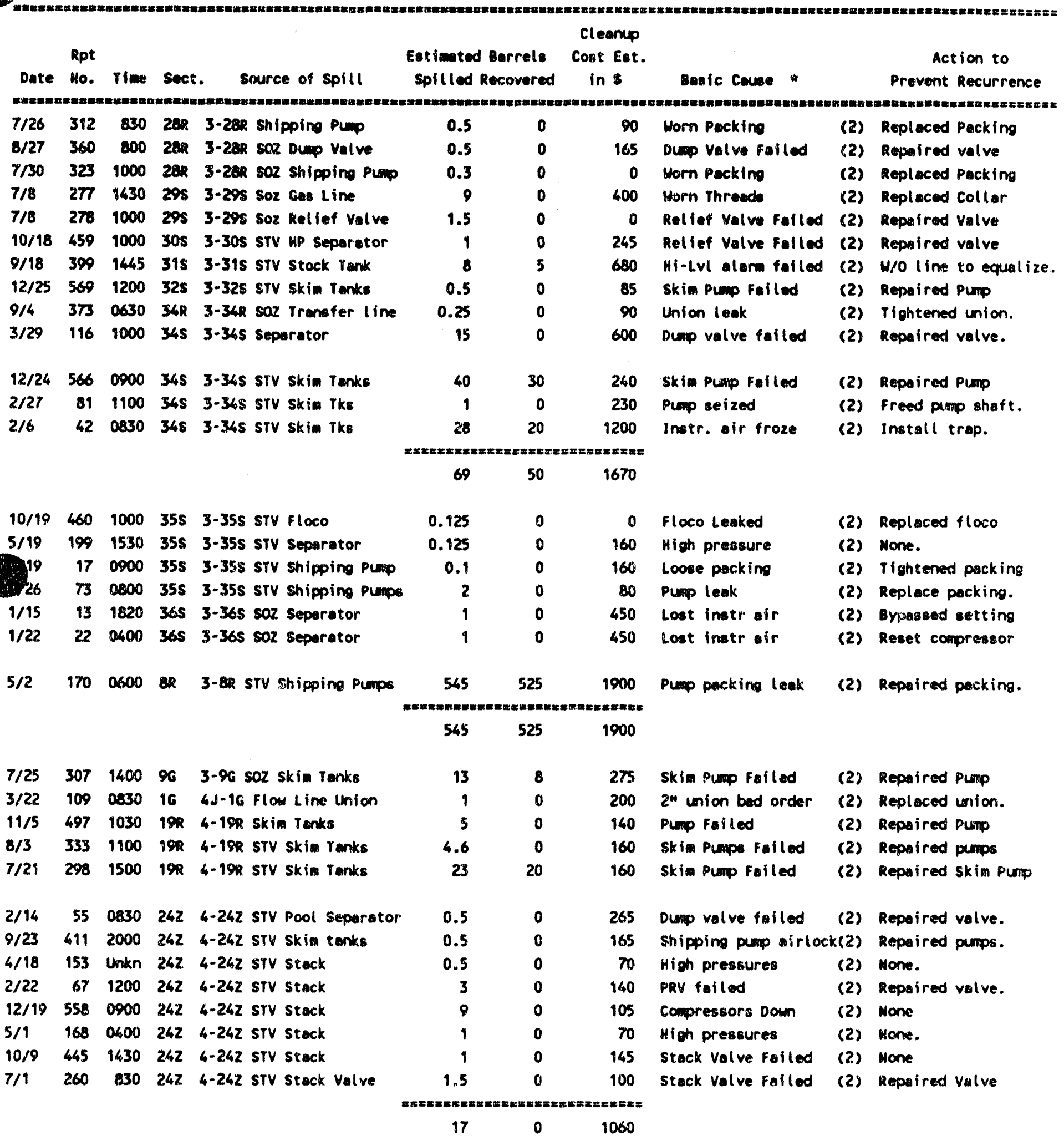

\footnotetext{
- (1) corrosion (2) mechanical fallure (3) Stuffing box (4) hlmun error
} 
OIL SPILLS OURIMG 1989

\begin{tabular}{|c|c|c|c|c|c|c|c|c|c|c|}
\hline \multirow{2}{*}{$\begin{array}{l}\text { Date } \\
=2 x=x=x \\
10 / 10\end{array}$} & $\begin{array}{l}\text { Rpt } \\
\text { No. }\end{array}$ & Time & sect. & source of spill & $\begin{array}{l}\text { Eetionter } \\
\text { spilled }\end{array}$ & overele & $\begin{array}{c}\text { Cleanup } \\
\text { Cost Est. } \\
\text { in } \$\end{array}$ & Dasic Ceuse & \multicolumn{2}{|r|}{$\begin{array}{l}\text { Action to } \\
\text { Prevent Recurrence }\end{array}$} \\
\hline & 450 & 1915 & 205 & 4-28s soz stock Tank & 1 & 0 & 165 & Hole in Tent & (2) & Penaired tant \\
\hline $9 / 26$ & 615 & 1200 & 29 & 4-29R STV Skim Tenks & 5.9 & 5.6 & 235 & Hi-LVl owitch failed & (2) & Repaired suitch. \\
\hline $12 / 22$ & 562 & 0200 & 328 & 4-32s sTV Relief Valve & 0.25 & 0 & 245 & Compreseors Doun & (2) & Mone \\
\hline $10 / 30$ & 487 & 9045 & 328 & 4-32s STV Relief Volve & 1 & 0 & 185 & Relief Valve Falled & (2) & None \\
\hline $12 / 24$ & 568 & 1010 & 325 & 4-32s STV Relief Volve & 3 & 0 & 165 & Relief Volve Failed & (2) & Adjueted Stack Valve \\
\hline $12 / 25$ & 578 & 1400 & 335 & 4-33s STV Skim Tenks & 2 & 0 & 280 & Skin Pump Failed & (2) & Repoired Punp \\
\hline $12 / 99$ & 556 & 1600 & 335 & 6-33s Test Separator & 0.0625 & 0 & 50 & Floco failed & (2) & None \\
\hline $9 / 22$ & 408 & 0500 & 345 & 4-345 STV poi gouge & 2 & 0 & 230 & Gouge B/O & (2) & Repluced gauge. \\
\hline $12 / 1$ & 520 & 1600 & 345 & 4-34S STV Shipping Pup & 0.125 & 0 & 50 & Nipple Mroke & (2) & Replaced Nipple \\
\hline $12 / 31$ & 584 & 1400 & 345 & 4-34S sTV Shipping Pump & 3 & 0 & 95 & Wipple Broke & (2) & Replaced Hipple \\
\hline $12 / 28$ & 580 & 0830 & 345 & 4-365 STV Shipping Pump & 0.125 & 0 & 0 & Mipple Broke & (2) & Replaced Nipple \\
\hline $4 / 29$ & 966 & 1600 & $35 R$ & 4-35R STV Relief Valve & 0.25 & 0 & 160 & Dump valve bed order & (2) & Repaired valve. \\
\hline $8 / 13$ & 346 & 900 & $35 R$ & 4-35R STV Shipping Puips & 0.5 & 0 & 165 & Pump Vibration & (2) & Installed charge pump \\
\hline $3 / 5$ & 92 & 1600 & 36 & 4-36 502 scrubber & 0.3 & 0 & 200 & check volve failed & (2) & Repaired valve. \\
\hline $7 / 2.9$ & 296 & 700 & 36 & 4-36 SOZ Skim Tanks & 0.25 & 0 & 40 & Shipping Punp Failed & $(2)$ & Repaired Shipp. Pump \\
\hline $4 / 3$ & 127 & 1200 & 26 & 58-26 stuffing Box & 0.5 & 0 & 200 & Pollish rod partied & (2) & Replaced rod. \\
\hline $7 / 5$ & 264 & 430 & $30 R$ & 2-30R STV Relief Valve & 0.5 & 0 & 160 & Relief Valve failed & (2) & Repoired Volve \\
\hline 1216 & 531 & 0930 & 345 & isB 34S stuffing 80x & 1 & 0 & 95 & Horn Packing & (3) & Repecked Well \\
\hline $12 / 25$ & 564 & 0800 & 106 & $115 W-106$ stuffing $80 x$ & 0.33 & 0 & 120 & Worn Packing & (3) & Repacked Well \\
\hline$y / 15$ & 550 & 0800 & 106 & 11-106 stuffing Box & 0.5 & 0 & 95 & Horn Packing & (3) & Repocked Hell \\
\hline 28 & 32 & 1300 & 18 & 13-18 stuffing Box & 8 & 3 & 185 & Worn packing & (3) & Repscked well \\
\hline $3 / 1$ & 91 & Unkn & 285 & 15x-28s stuffing Box & 3 & 2 & 160 & Horn packing & (3) & Reploced pocking. \\
\hline $2 / 23$ & 69 & 0900 & $28 s$ & 15-28s stuffing $\operatorname{cox}$ & 0.125 & 0 & 45 & Horn packing & (3) & Repecked well. \\
\hline $6 / 3$ & 222 & 700 & 142 & $17-142$ stuffing Box & 1 & 0 & 160 & Worn Packino & (3) & Reprecked well. \\
\hline $6 / 1$ & 217 & 1900 & 142 & 17-142 Stuffing Box & 1 & 0 & 0 & Horn Packing & (3) & Repacked well. \\
\hline $6 / 13$ & 239 & 1000 & 142. & 17-142 Stuffing Box & 0.5 & 0 & 160 & Worn Pecking & (3) & Repacked well. \\
\hline $12 / 14$ & 546 & 1000 & 365 & 216-36s stuffing Box & 0.5 & 0 & 95 & Worn Pecking & (3) & Repecked Well \\
\hline $10 / 13$ & 453 & 1230 & 365 & 216-36s Stuffing Box & 7 & 5 & 70 & Horn Packing & (3) & Repacked well \\
\hline $3 / 1$ & 88 & 1000 & 345 & 21A-34s stuffing Box & 2 & 1 & 45 & Horn pecking & (3) & Reploced pocking. \\
\hline $8 / 25$ & 359 & 1200 & $35 s$ & 21x-35s stuffing Box & 2 & 1 & 95 & Horn Packing & (3) & Repacked Well \\
\hline $1 / 31$ & 37 & 1030 & 365 & 2215-36s Stuffing 80x & 1 & 0 & 120 & Horn pecking & (3) & Repocked well \\
\hline $7 / 26$ & 313 & 1000 & 365 & 2215-36s Stuffing Box & 2 & 8 & 90 & Worn Packing & (3) & Replsced Pocking \\
\hline $7 / 21$ & 303 & 1100 & $36 s$ & 2215-36s stuffing Box & 1 & 0.5 & 45 & Worn Packing & (3) & Repluced Packing \\
\hline $10 / 7$ & 439 & 0730 & 365 & 2215-36s stuffing sox & 1 & 0 & 95 & Worn Packing & (3) & Repacked well \\
\hline $6 / 18$ & 243 & 1000 & 345 & 22A 34s stuffing box & 4 & 2 & 300 & Horn Packino & (3) & Repacked well!. \\
\hline $9 / 16$ & 395 & 0800 & 345 & $24 n-34$ s stuffing $80 x$ & 4.5 & 2 & 165 & Horn pocking & (3) & Repocked well. \\
\hline $8 / 19$ & 354 & 930 & 34,5 & $24 A-345$ stuffing $80 x$ & 0.5 & 0 & 95 & Horn Packing & (3) & Repacked Hell \\
\hline $5 / 18$ & 126 & 9100 & 298 & $24-29$ s stuffing $80 x$ & 2 & 0 & 140 & Worn packing & (3) & Reploced packing. \\
\hline $7 / 5$ & 261 & 1100 & $28 R$ & 26-28R Stuffing Box & 1 & 0 & 900 & Worn Packing & (3) & Replsced Pocking \\
\hline $3 / 2$ & 8 & 0800 & $28 R$ & 26-28R stuffing Box & 6 & 5 & 200 & Horn packing & (3) & Replaced packing. \\
\hline $7 / 25$ & 309 & 730 & $29 R$ & 26-29R stuffing $80 x$ & 0.125 & 0 & 160 & Worn Packing & (3) & Replaced Packing \\
\hline $9 / 13$ & 391 & 1930 & $28 s$ & 27-28S Stuffing Box & 1.5 & 0 & 120 & Worn packing & (3) & Repocked well. \\
\hline $2 / 24$ & 78 & unkn & $2 \pi R$ & 28-27R stuffing Box & 2 & 0 & 230 & Worn packing & (3) & Repacked well. \\
\hline $5 / 9$ & 186 & 1000 & $27 s$ & 28-27s stuffing $20 x$ & 1 & 0 & 90 & Horn peckino & (3) & Reploced packing. \\
\hline & 95 & 1130 & $2 / R$ & 2-27R STV Shipping Pump & 1 & 0 & 160 & Packing leak & $(3)$ & Replaced pocking. \\
\hline
\end{tabular}

\footnotetext{
- (1) corrosion (2) mechanical fallure (3) STUfFing goX (4) mLMan error
} 
OIL EPILLS DURIMG 1989

\begin{tabular}{|c|c|c|c|c|c|c|c|c|c|c|}
\hline \multirow{2}{*}{$\begin{array}{l}\text { Date } \\
\text { mangmax }\end{array}$} & $\begin{array}{l}\text { Rpt } \\
\text { Mo. }\end{array}$ & Time & $\operatorname{sect}$ & source of spill & \multicolumn{2}{|c|}{$\begin{array}{l}\text { Estimated Barrels } \\
\text { spilled Recovered }\end{array}$} & $\begin{array}{l}\text { Cleormp } \\
\text { Cost Est. } \\
\text { in } \$\end{array}$ & Basic Cause" & \multicolumn{2}{|r|}{$\begin{array}{c}\text { Action to } \\
\text { Prevent Recurrence }\end{array}$} \\
\hline & 355 & 400 & 262 & $311-262$ stuffing box & 0.5 & 0 & 230 & Worn Packing & (3) & Repecked Well \\
\hline $1 / 13$ & 10 & 1000 & $1 m$ & 313-17R stuffing cox & 1.5 & 0 & 230 & Horn packing & (3) & Repacked well \\
\hline $5 / 1$ & 171 & 0745 & 46 & 313-46 stuffing cox & 0.25 & 0 & 250 & Horn packiro & (3) & Replaced pocking. \\
\hline $11 / 26$ & 518 & 0900 & 348 & 315A-345 stuffind Box & 6 & 0 & 860 & Polfish Rod Broke & (3) & Replaced Pollish Rod \\
\hline $12 / 25$ & 572 & 9830 & $35 s$ & 316-35s stuffing Box & 0.5 & 0 & 135 & Horn Packing & (3) & Repacked Well \\
\hline $10 / 16$ & 458 & 1530 & $17 R$ & 322-17R Stuffing Box & 1.5 & 0 & 235 & Morn Packing & (3) & I epacked well \\
\hline $12 / 15$ & 547 & 0800 & 188 & 331-18R Stuffing Box & 0.33 & 0 & 165 & Worn Packing & (3) & Wepacked Well \\
\hline $4 / 99$ & 154 & 1030 & 8 & 337-8R Stuffing Box & 1 & 0 & 140 & Horn peckino & (3) & Reploced packing. \\
\hline $7 / 19$ & 292 & 1100 & $8 n$ & 337-8R stuffing Bax & 0.5 & 0 & 160 & Worn Pecking & (3) & Reploced Pocking \\
\hline $7 / 99$ & 293 & 1845 & 355 & 338-35s stuffing Box & 2 & 0 & 240 & Worn Packing & (3) & Replocod Packing \\
\hline $10 / 8$ & 437 & 9030 & 345 & 33A-34S Stuffing Box & 0.5 & 0 & 95 & Horn Packing & (3) & Repacked well \\
\hline $7 / 23$ & 306 & 200 & 26 & 342-2C stuffing Bax & 3 & 0 & 700 & Horn Pocking & (3) & Reploced Packing \\
\hline $11 / 16$ & 519 & 0800 & 325 & $346 A-325$ stuffing Box & 1.5 & 0 & 140 & Horn Packing & (3) & Repecked Well \\
\hline $4 / 19$ & 156 & Unkn & $33 R$ & $344-33 R$ Stuffing Box & 0.25 & 0 & 160 & SB threads leak & (3) & Replace SB. \\
\hline $6 / 16$ & 244 & Unkn & $33 R$ & $364-33 R$ Stuffing Box & 1 & 0 & 240 & Horn Packing & (3) & Repocked well. \\
\hline $2 / 27$ & 82 & 0630 & 335 & $344-335$ stuffing Box & 10 & 0 & 1200 & Polish rod perted & (3) & Reploced rad. \\
\hline $2 / 19$ & 66 & 0500 & $28 R$ & 346-28R Stuffing Box & 7 & 6 & 250 & Horn packing & (3) & Repacked well. \\
\hline $2 / 24$ & 79 & 0700 & 142 & $355-142$ stuffing dox & 4 & 0 & 160 & Horn pocking & (3) & Repseked well. \\
\hline $8 / 7$ & 337 & 600 & $35 s$ & 355-35s stuffing Box & 0.25 & 0 & 150 & Horn Pocking & (3) & Repacked Well \\
\hline & 16 & 1300 & $34 R$ & 356-36R Stuffind Box & 0.5 & 0 & 230 & Horn packing & (3) & Repocked well \\
\hline$\sqrt{3}$ & 50 & 1400 & $8 R$ & 357-8R stuffing Box & 1 & 0 & 370 & Worn packing & (3) & Repacked well. \\
\hline $8 / 7$ & 335 & 800 & 132. & $358 x 132$ stuffing sox & 2 & 0 & 185 & Horn Pocking & (3) & Repacked Hell \\
\hline $2 / 14$ & 54 & 1030 & 932 & $358 x-132$ stuffin Box & 14 & 12 & 550 & Worn packing & (s) & Repacked well. \\
\hline $7 / 2$ & 262 & 900 & $35 s$ & 350-35s stuffing 80x & 5 & 3 & 90 & Worn Packing & (3) & Replacod Packing \\
\hline $6 / 18$ & 265 & Unkn & $33 s$ & 366-33s stuffing Box & 0.25 & 0 & 300 & Worn Packins & (3) & Repacked well. \\
\hline $7 / 21$ & 300 & 830 & 345 & 366-345 Stuffing Box & 9 & 4 & 230 & Bridle Broke & (3) & Reploced Bridle \\
\hline $1 / 97$ & 15 & 0900 & 345 & 366-34s Stuffing 80x & 20 & 15 & 2400 & Polish rod perted & (3) & Reploced polish rod \\
\hline $5 / 18$ & 195 & 1000 & 305 & 367-30s Stuffing Box & 1 & 0 & 140 & Loose packing & $(3)$ & Tightened pocking. \\
\hline $4 / 21$ & 161 & 0800 & 325 & 368M-32s stuffing Box & 2 & 0 & 140 & Horn pecking & (3) & Reploced pocking. \\
\hline $9 / 13$ & 392 & 0800 & 345 & $368-345$ stuffing eox & 0.5 & 0 & 190 & Worn packing & (3) & Repacked well. \\
\hline $2 / 27$ & 84 & 1200 & 365 & $36 x-365$ stuffing Box & 1 & 0 & 45 & Celler overflow & (3) & Pull cellar. \\
\hline $7 / 11$ & 281 & 900 & 298 & $373-292$ stuffing Box & 1.2 & 0 & 160 & Worn Packing & (3) & Replaced Pocking \\
\hline $12 / 17$ & 549 & 0300 & 262 & $374-262$ stuffing Box & 6 & 0 & 165 & Morn Packing & (3) & Repociked Well \\
\hline $7 / 16$ & 291 & 830 & 60 & 374.66 stuffing $B O x$ & 2 & 0 & 180 & Worn Packing & (3) & Replaced Pocking \\
\hline $12 / 6$ & 5.22 & 0600 & 66 & $374-66$ stuffing Box & 2 & 0 & 165 & Worn Packing & (3) & Repocked Well \\
\hline $8 / 29$ & 366 & 900 & 60 & $374-60$ stuffing Box & 2. & 0 & 190 & Worn Packin & (3) & Repocked Hell \\
\hline $10 / 19$ & 463 & 1100 & 66 & $374-66$ stuffing $80 x$ & 2 & 0 & 165 & Worn Packing & (3) & Repacked well \\
\hline $6 / 4$ & 223 & 2130 & $28 R$ & 386-28R stuffing Box & 2 & 0 & 220 & Worn Packino & (3) & Repacked well. \\
\hline $11 / 6$ & 499 & $\$ 045$ & 26 & 38-2G stuffing Box & 1.5 & $0 . \sqrt{5}$ & 70 & Worn Packing & (3) & Repocked Hell \\
\hline $4 / 20$ & 150 & 0900 & 305 & $42-30$ S stuffing Box & 1.5 & 0 & 160 & Worn packing & (3) & Replaced packing. \\
\hline $10 / 23$ & 669 & 1800 & 335 & $43 x-33$ s stufting Box & 2 & 0 & 165 & Worn Packing & (3) & Repacised well \\
\hline $3 / 18$ & 105 & 2800 & $28 s$ & 45-28s seufting Bax & 5 & 0 & 370 & Worn pack ing & (3) & Reploced pocking. \\
\hline $4 / 9$ & 137 & 0030 & 285 & 45-28S stuffing Box & 2 & 0 & 320 & Horn packing & (3) & Replaced packing. \\
\hline $2 / 23$ & 70 & 1000 & 285 & 45-28s stuffing Box & 0.125 & 0 & 0 & Worn packing & (3) & Repacked well. \\
\hline & 28 & 0800 & 106 & 46-106 stuffing Box & 9 & 7 & 160 & Worn pecking & (3) & Repecked well \\
\hline
\end{tabular}

- (1) corrosion (2) Mechanical fallure (3) STUfFing gox (4) human errok 
OIL SPILLS DURIMG 1989

\begin{tabular}{|c|c|c|c|c|c|c|c|c|c|c|}
\hline \multirow{2}{*}{$\begin{array}{l}\text { Dote } \\
=m=m a n= \\
6 / 26\end{array}$} & $\begin{array}{l}\text { Rpt } \\
\text { Ao. }\end{array}$ & Tine & $\operatorname{sect}$. & source of splll & \multicolumn{2}{|c|}{$\begin{array}{l}\text { Estineted Barrels } \\
\text { spllled Recovered }\end{array}$} & $\begin{array}{l}\text { Cost Est. } \\
\text { in } 8\end{array}$ & Besic Ceuse & \multicolumn{2}{|r|}{ 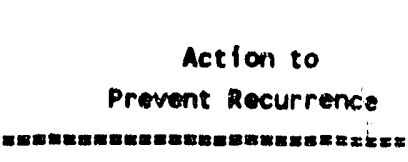 } \\
\hline & 251 & 1230 & 362 & 48-36R stuffing $80 x$ & 2.25 & 2 & 140 & \multirow{2}{*}{$\begin{array}{l}\text { Worn Packing } \\
\text { Worn packing }\end{array}$} & \multirow{2}{*}{$\begin{array}{l}\text { (3) } \\
\text { (3) }\end{array}$} & \multirow{2}{*}{ Repacked well. } \\
\hline $2 / 26$ & 74 & 1900 & 348 & 53-265 stuffing Bax & 0.125 & $\mathbf{0}$ & 45 & & & \\
\hline $6 / 8$ & 235 & 1000 & 305 & 54-30s stuffing Bax & 3 & 0 & 190 & Worn Packing & (3) & Repacked mell. \\
\hline $8 / 2$ & 330 & 900 & $30 \pi$ & 55-300 stuffing dax & 1.4 & 0 & 120 & Worn Packing & (3) & Repecked Mell \\
\hline $2 / 23$ & 71 & 0900 & 16 & 56-1G stuffing Box & 3 & $\mathbf{0}$ & 160 & Worn pecking & (3) & Repecked well. \\
\hline $6 / 26$ & 255 & 900 & 262 & 584-262 Stuffing sox & 0.25 & 0 & 160 & Horn Packing & (3) & Repacked well. \\
\hline $10 / 3$ & 430 & 0800 & 26 & 58-26 stuffing $80 x$ & 0.25 & 0 & 235 & Worn Packin & (3) & Repacked well \\
\hline $6 / 3$ & 226 & 800 & 142 & $5-344-142$ stuffing Dox & 1 & 0 & 160 & Horn Packing & (3) & Repecked well. \\
\hline $6 / 27$ & 258 & 1445 & 142 & $5-364-142$ stuffing Box & 5 & 3.5 & 230 & Horn Peckin & (3) & Repacked well. \\
\hline $5 / 3$ & 174 & 1130 & 142 & 5-344-142 stuffing Box & 0.5 & 0 & 230 & Horn pecking & (3) & Repleced packing. \\
\hline $9 / 21$ & 406 & 0800 & $35 s$ & 60-35s stuffind Box & 0.5 & 0 & 140 & Worn packing & (3) & Repacked well. \\
\hline $4 / 20$ & 157 & 0800 & 305 & 62-30s stuffing Box & 1 & 0 & 45 & Worn packing & (3) & Replaced packing. \\
\hline $7 / 30$ & 317 & 1400 & 365 & 62-36s stuffing Box & 2 & 1 & 230 & Horn Packing & (3) & Replaced Packing \\
\hline $3 / 29$ & 117 & Untkn & $1 B$ & 64-1B stuffing Box & 6 & 0 & 400 & Norn pecking & (3) & Replaced pocking. \\
\hline $5 / 2$ & 184 & 1000 & 295 & 65-29s Stuffing Box & 2 & 1 & 45 & Horn pecking & (3) & Replaced pocking. \\
\hline $4 / 15$ & 147 & 1600 & 295 & 65-295 Stuffing Box & 1.75 & 0 & 160 & Horn packing & (3) & Replaced packing. \\
\hline $4 / 9$ & 136 & 0900 & $28 s$ & $66-205$ stuffing Box & 4 & 0 & 170 & Horn packing & (3) & Repleced packing. \\
\hline $2 / 8$ & 46 & 0800 & $34 \mathrm{~S}$ & 6-345 stuffing Box & 4 & 0 & 80 & Worn packing & (3) & Repacked well. \\
\hline $5 / 31$ & 218 & 1330 & 305 & 72-30s stuffing Bax & 1 & 0 & 90 & worn packing & (3) & Replaced pacting. \\
\hline 45 & 41 & 1030 & 20 & $75-26$ stuffing Box & 7 & 2 & 160 & Polish rod parted & (3) & Replaced rod. \\
\hline 19 & 96 & 0000 & 355 & 75-35s stuffing Bax & 0.5 & 0 & 80 & Worn Packing & (3) & Replaced packing. \\
\hline $3 / 1$ & 87 & 2400 & 355 & $75-35 s$ stuffing Box & 1 & 0 & 120 & Worn Packing & (3) & Repleced packing. \\
\hline $11 / 11$ & 505 & 0500 & 295 & 76-295 stuffing Box & 5 & 0 & 70 & Horn Packing & (3) & Repocked thell \\
\hline $5 / 29$ & 211 & 0900 & $33 R$ & 76-33R stuffing Box & 9.5 & 0 & 250 & Worn packing & (3) & Replaced pocking. \\
\hline $8 / 16$ & 348 & Unkn & $35 R$ & $77-35 R$ stuffing $80 x$ & 0.5 & 0 & 95 & Horn Packing & (3) & Repacked Hell \\
\hline $4 / 13$ & 143 & 16,30 & $35 R$ & $\pi$-35R stuffing Box & 0.5 & 0 & 160 & Horn packing & (3) & Replaced packing. \\
\hline $7 / 13$ & 287 & 1300 & $35 R$ & 77-35R stuffing 8ox & 1 & 0 & 160 & Worn Packing & (3) & Replacod Packing \\
\hline $3 / 3$ & 90 & 1130 & 96 & $78 x-96$ stuffing $B 0 x$ & 3 & 2 & 160 & Horn packing & (3) & Repleced packing. \\
\hline $1 / 13$ & 11 & 1030 & 60 & $78-66$ stuffing tox & 5 & 0 & 140 & Worn pocking & (3) & Repocked well \\
\hline $9 / 4$ & 376 & 0500 & 96 & $81-96$ seufting Box & 3.5 & 0 & 275 & Loose packing & (3) & Tightened packing. \\
\hline $9 / 21$ & 407 & 1400 & 16 & 84-16 stuffing Box & 1 & 0 & 95 & Worn packing & (3) & Repackert well. \\
\hline $4 / 20$ & 158 & Unkn & $24 R$ & Q $-26 R$ stuffing Box & 3 & 0 & 160 & Worn packing & (3) & Replaced packing. \\
\hline $2 / 1$ & 38 & 1100 & $26 R$ & 84-24R stuffing Box & 2 & $\mathbf{0}$ & 130 & Cellar overflowed & (3) & Repacked well. \\
\hline $5 / 23$ & 205 & 0700 & $33 R$ & 84-33R stuffing Box & 1 & 0 & 90 & Worn packing & (3) & Replaced packing. \\
\hline $19 / 1$ & 492 & 0700 & 46 & QBNE-4G Stuffing 80X & 1 & 0 & 165 & Worn Psecking & (3) & Repecked Hell \\
\hline $12 / 18$ & 559 & 1130 & 986 & 186 LACT Drain Line & 1 & 0 & 0 & Backhoe Mit Line & (4) & Reploced Line \\
\hline $6 / 23$ & 250 & 1500 & 186 & 186 Lact Settling rank & 50 & 40 & 600 & Line Not Blinded & $(4)$ & Installed blind \\
\hline & & & & & $z:=x=x=x$ & $=2 x=x$ & $u ; z x=z=z=2$ & & & \\
\hline & & & & & 50 & 40 & 600 & & & \\
\hline $1 / 31$ & 40 & 1100 & $17 R$ & 1-17R STV Shippind Pumps & 6 & 0 & 140 & Loose nut ofter PMI & $(4)$ & Tightened nut \\
\hline $9 / 22$ & 404 & 0800 & $25 R$ & 1-25R STV Drain Box & 0.1 & 0 & 40 & Opened valve & $(4)$ & closed valve. \\
\hline $12 / 18$ & 552 & 1000 & $32 \mathrm{~s}$ & 1-32S STV Drain & 0.25 & 0 & 95 & Drsin Overflawed & (4) & Counselled Operator \\
\hline & 227 & 1200 & 255 & 25S Lact Tank $\$ 372$ & 0.25 & 0 & 90 & Front Looder Hit Lin & e(4) & Repaired line \\
\hline
\end{tabular}

\footnotetext{
- (1) corrosidn (2) mechanical fail.ure (3) STUfFing boX (4) Muman error
} 
OIL SPILLS DURING 1989

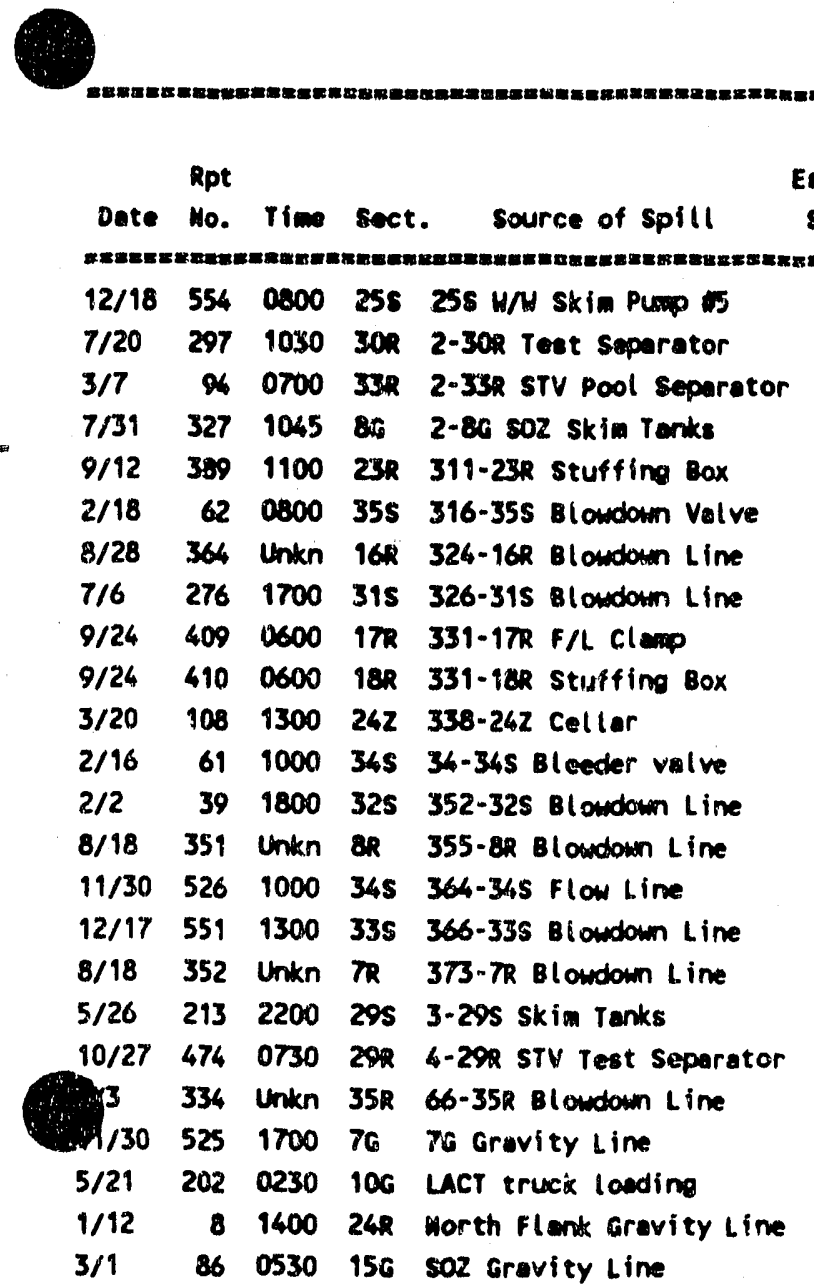

\section{Cleenup}

Estimated Barrels Cost Est.

\begin{tabular}{|c|c|c|c|c|c|}
\hline 1 & 0 & 95 & Valve Left open & $(4)$ & closed Valve \\
\hline 0.125 & 0 & 115 & Valve left open & $(4)$ & Closed Valve \\
\hline 2 & 0 & 160 & Drain valve open & (4) & closed valve. \\
\hline 4 & 0 & 210 & Compressor Volve S/I & $(4)$ & Opened Valve \\
\hline 1 & 0 & 200 & Valve shut at setting & $(4)$ & Opaned valve. \\
\hline 8 & 4 & 275 & Valve left open & $(4)$ & closed valve. \\
\hline 2 & 1.5 & 220 & Valve Left Open & (4) & closed valve \\
\hline 0.25 & $\mathbf{0}$ & $\infty$ & Valve Left open & (4) & closed Valve \\
\hline 2 & 0 & 95 & Volve shut-in & $(4)$ & Opened valve. \\
\hline 9 & 0 & 580 & Valve shut-in & $(4)$ & Opened valve. \\
\hline 2.5 & 2 & 80 & Ceg valve left open & (4) & closed valve. \\
\hline 4 & 2 & 90 & Valve left open & (4) & closed valve. \\
\hline 0.25 & 0 & 45 & Depreasurized well & (4) & Counsel ad oper. \\
\hline 0.5 & 0 & 95 & Valve Left open & $(4)$ & closed valve \\
\hline 1 & 0 & 70 & Backhoe Hit Line & (4) & Clemped Lirve \\
\hline 0.125 & 0 & 140 & Valve left open & (4) & closed Valve \\
\hline 0.25 & 0 & 95 & Valve left Open & (4) & Closed volve \\
\hline 0.5 & 0 & 160 & Drain valve left open & $n(6)$ & closed valve. \\
\hline 6 & 0 & $7 \%$ & Discharge Valve $s / I$ & (4) & Qponod valve \\
\hline 2 & 0 & 100 & Valve Left open & (4) & closed valve \\
\hline 6 & 5 & 200 & Gas Vent Left Open & (4) & Closed Vulve \\
\hline 4.5 & 4 & 140 & Valve left open & $(4)$ & cloeed valve. \\
\hline 5 & 0 & 330 & Vent left open & (4) & closed vent \\
\hline 30 & 8 & 350 & i/2n val ve opened & (4) & Valve locked out. \\
\hline
\end{tabular}
spillied Reciovered in 8

$\ln 8$

Action to

Basic Cause

Prevent Recurrence

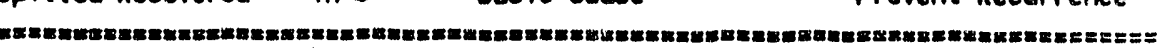

(4) Volve locked out. 


\section{APPENDIX F}

SUMMARY OF 1989 HAZARDOUS WASTE SHIPMENTS FROM NPR-1 AND 1989 FACILITY ANNUAL HAZARDOUS WASTE REPORT TO THE CALIFORNIA DEPARTMENT OF HEALTH SERVICES 


\section{TABLE OF CONTENTS}

\section{APPENDIX F}

I. Hazardous Waste Facility Report for 1989 (submitted to the California Department of Health Services)

II. Summary of Hazardous Waste Manifest Data for 1989 (submitted to the California Department of Health Services) 
28590 Highway 119

Tupman. California

Mall Address: P.O. Box 127. Tupman, CA 93276

Telephone: (805) 763-60100

Department of Health Services

714/744 P street

P.O. Box 942732

Sacramento, CA 93234-7320

Subject: 1989 HAZARDOUS WASTE REPORT

Gentlemen:

Attached are the 1989 Hazardous waste Report forms IC

(Identification and Certification) and GM (waste Generation and Management) for the liaval Petroleum Reserve No. 1 (NPR-1), EPA number CA4170024414, as required by Title 22 , California Code of Regulation section 66493 and/or section 67165.

NPR-1 is an operating oil field which produces waste in association with well drilling and the production of oil and gas. predominatous wastes generated and disposed of off site consist contaminated soil.

NPR-1 does not receive any hazardous waste from off site and no hazardous wastes are treated, disposed of or recycled on site. Therefore forms WR (Waste Received from off site) and PS (Waste Treatment, Disposal or Recycling Process System) did not require
completion.

$\begin{array}{ll}\quad \text { PDOH } & \text { Sincerely, } \\ \text { REC/BDH:rbe } & \text { Manager, Environmental } \\ \text { Attachment } & \text { Services } \\ \text { CC: DNPRC } & \end{array}$


BLFORE COPYING FOAM, ATTACH SITE IOENTIFICATION LABEL
OR ENTER:

$\checkmark$

SITE NAME

-

Elk Hills Naval Petroleum Reserve Number 1

EPAIDNO

$\left|C 1^{A}, 4, y^{7}, 0,02,1^{4}, 4,1,4\right|$

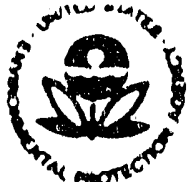

FORM
U.S. ENVIRONMENTAL

PROTECTION AGENCY

1989 Hazardous Waste Report

IDENTIFICATION AND

CERTIFICATION

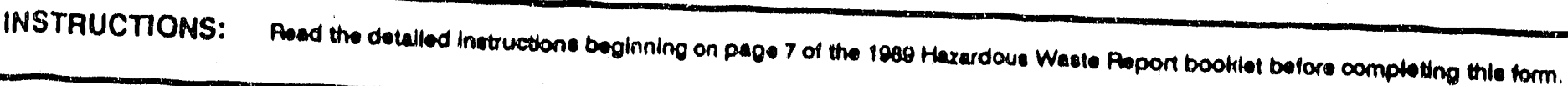

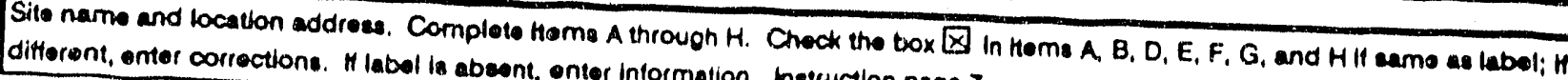

A. EPA 10 No.

different, enter correction. H labal is abeent, onter Information. instruction page 7.

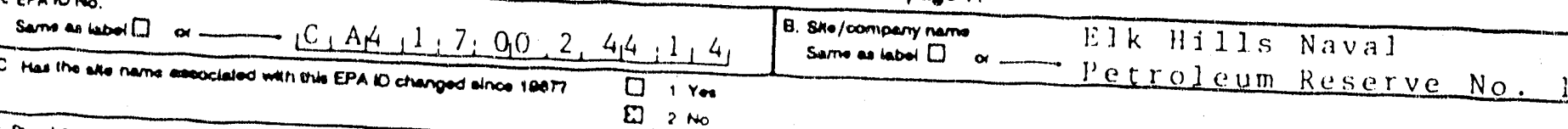

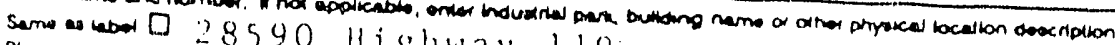

o. 2590 ll iglliwa 119

F Cry, lown, whang, atc

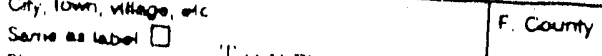

$\cdots$

'Tu pri: :

Ki l $1:$.

o. Sine

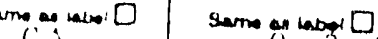

SEC. II Mailing addrese of site. Inetruction page 7.

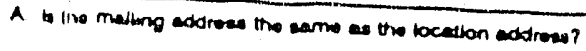

D Val (SNOP TO SEC. MI)

Aumber and wrod name of maling acioive

(1) 2 NG IDOMPLTE SEC. II

(). $\mathrm{BO} \times 127$

C. Con, lown, Whage, atc

Tupman

D. siate

CA $A$

E. $210 \cos 0$

SEC. III

Name, titte, and telephone number of the porson who should beon

(1)

$L^{9}+3,2,7,6-1+1,1$

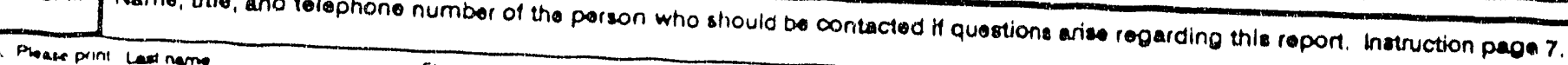

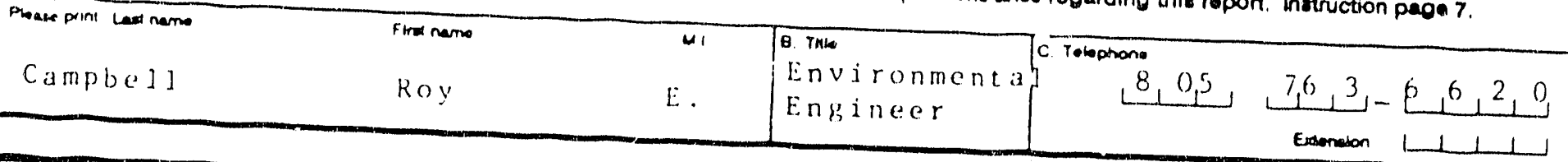

SEC. $N$

Enter the Stendard indurtrial Geselfication (SIC) Code that doscribes the principal prod

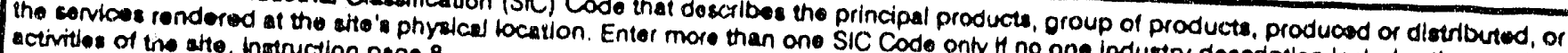
activities of the atte. inetruction page 8 .

a
$1,3,11$
$1+3,8,1$
$1,3,8,2$
1131819

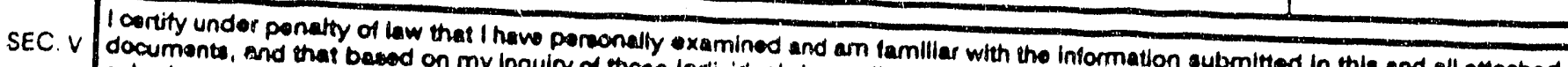

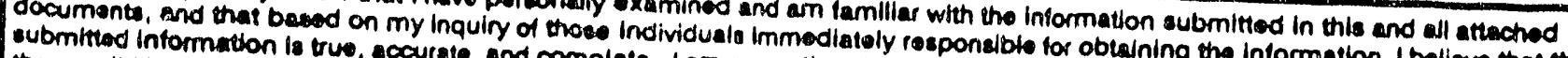

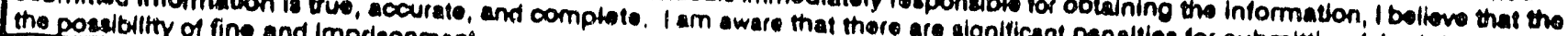

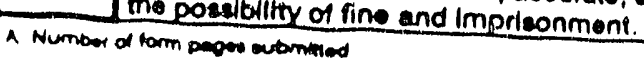

Formic 1212$)$ Form on 110

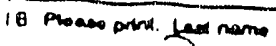

form Wr

لــــ

form Pe

M.I

$<$

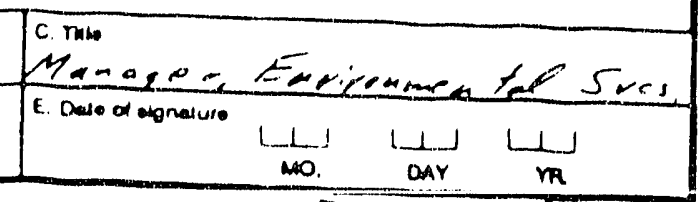

Page 1 of

PA form 8700 1348 (5-80)

(Roviead 11-85) (Alovined 12-87) (Roviend 11-89)

OVEA $\rightarrow$ 


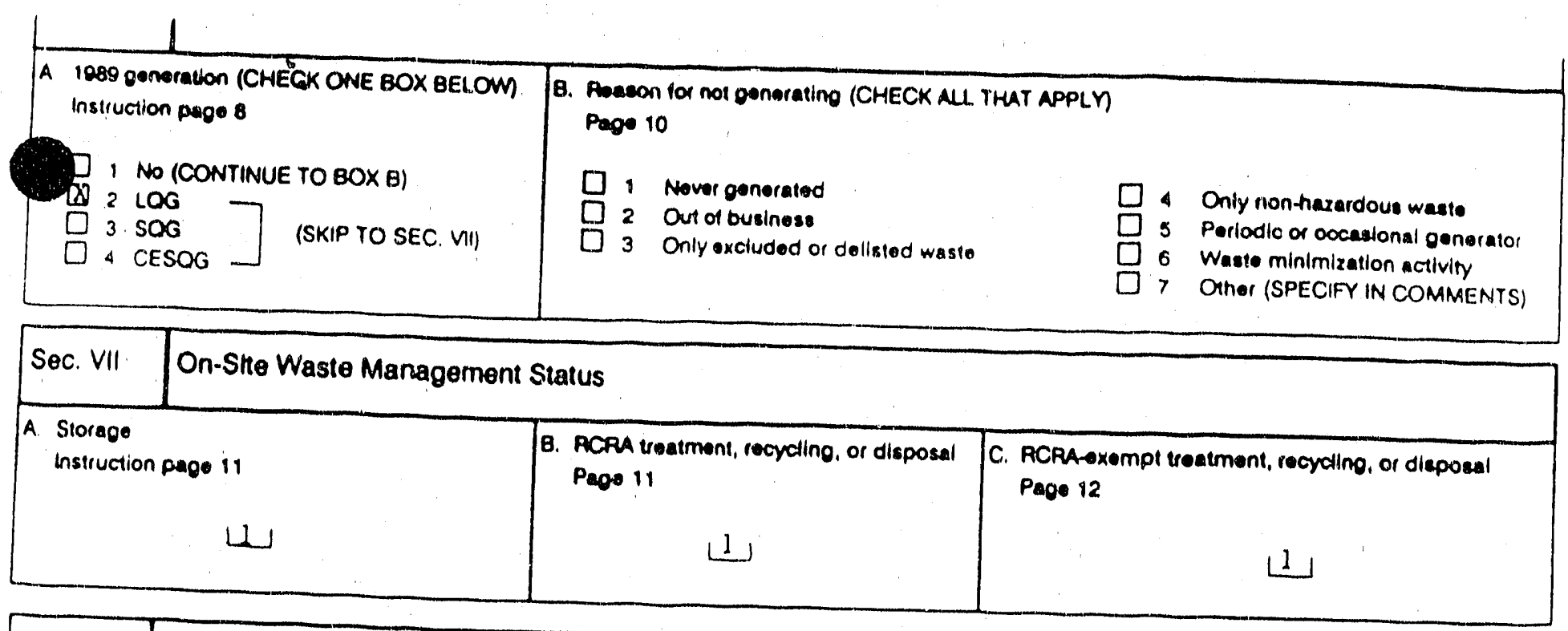

\begin{tabular}{|l|l|l|}
\hline Sec VIII & Waste Minimization Activity during 1988 or 1989 \\
\hline
\end{tabular}

A. Did this sito begin or expand a source ceductua activity during 1088 or 1899 ? insiruction page 12

[.], Yes

$\square 2$ No
8. Did this site begin or expend a cecexcling activity during 1988 or 1989 ? Page 13

D, Yes

D 2 No
C. Did this site conduct sourow reduction or recyaling

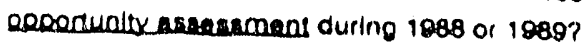
Pag. 13

, Yo6

¿. No OO What lactors have limitod this site from initiating new source reduction activities during 1988 or 1909 ?
(CHECK ALL THAT APPLY)

$\square$ No factors have limiled now sourco reduction activities

$\square 03$ Lack of technical intormation install new source reduction oquipment or implement now sourca reduction practices.

$\square$ 04 Source reduction tormation on source reduction techniques applicable to the spoctic production procesese

$\square$ os Concorn that product qualtey may dectine

[0 06 Technical limitations of the production procosses.

$\square$ Tectinical limitations of the production processes.

$\square 07$ Permitting burdens.

08 Other (SPECIFY IN COMMENTS)

E. What factors have limited this she from initiating now on-site or off-site coracking activities during 1888 or 1989 ? ICHECK ALL THAT APPLY

Page 13

X8 01 No lactors have limitod now recycting activitios.

02 Insutficient capital to install now rocycling equipment or implement now recpclirio practices.

03 Lack of technical intormation on rocycling techniques applicuble to this site' epecific production processes

04 Rocycling not oconomically toasible: cost auvings in wast management or production will not recover the capital imvestment.

05 Conoern that product qualty may docline as a cesuth of recycting.

D6 Requirements to manifert wastes inhibit shipments oft site for rocycling.
07 Financial liability provisions inhibit ahlpments oft alte for recycling.

$\square$ as Technical limitations of product procesees inhiblt Ehipments oft site for recycling.

09 Techical limitations of production proceeses inhibit on-eite recycling

[ 10 Permitting burden: inhibit recycting.

$\square$ is Lack of permitted oft-ette recycling taciltiles.

[ 12 Unable to identity a market for recyctable materials.

$\square$ 13 Other (SPECIFY IN COMAENTS)

roming:

omments: 


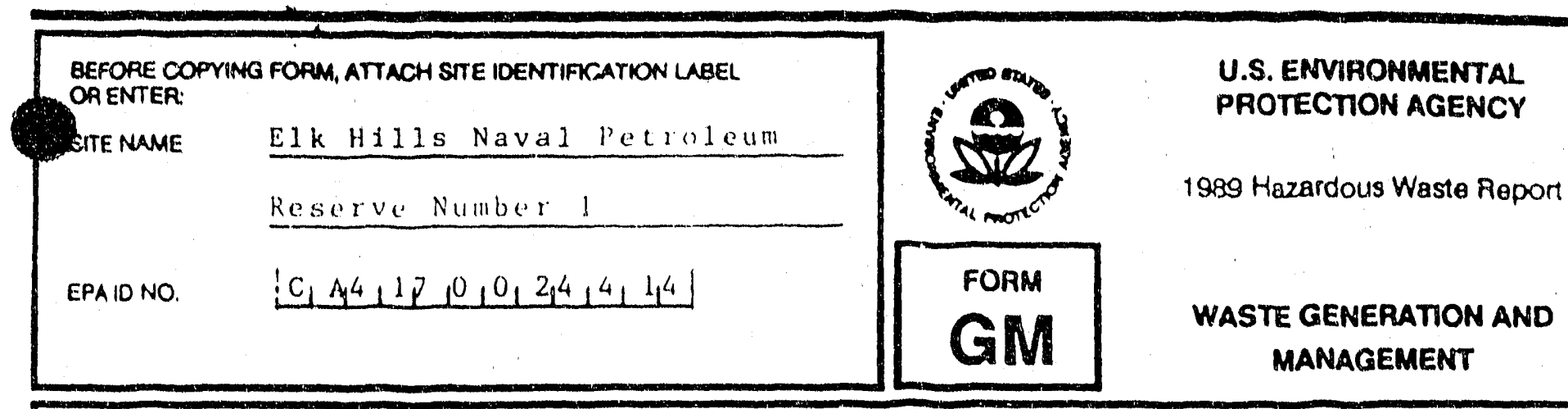

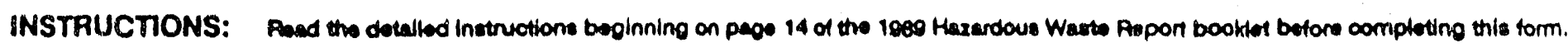

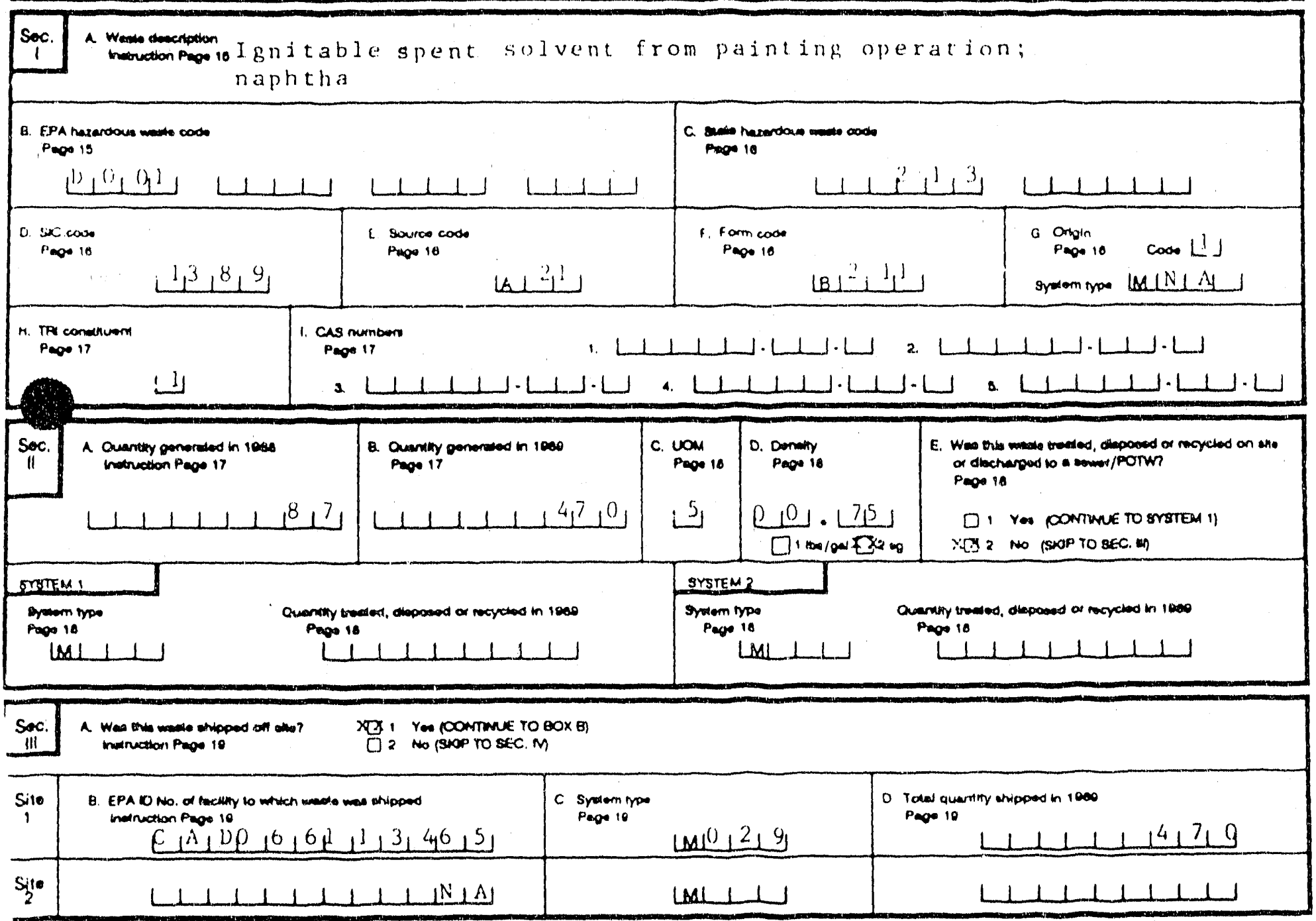

\begin{tabular}{|c|c|c|c|c|c|}
\hline \multirow{2}{*}{$\begin{array}{c}\text { Soc. } \\
\frac{N}{B}\end{array}$} & \multicolumn{2}{|c|}{ 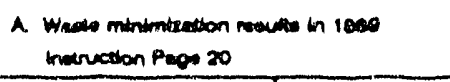 } & \multicolumn{3}{|c|}{ 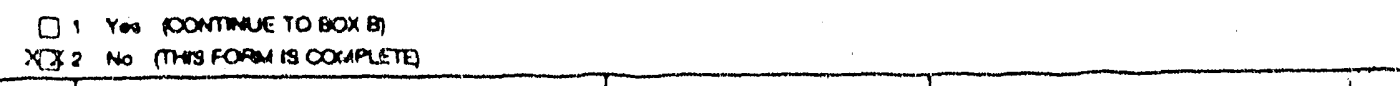 } \\
\hline & $\operatorname{sinyy} 21$ & 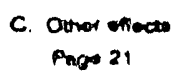 & 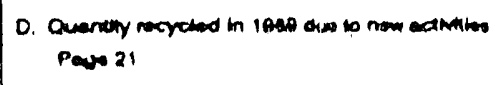 & 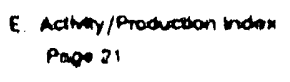 & $\begin{array}{l}\text { F. Source Production Cumantry } \\
\text { Pmos zas }\end{array}$ \\
\hline & $|w|$ & 0, Yor & $\mid$\begin{tabular}{l|l|l|l|l|l|l|l|l|l|l}
1 & 1 & 1
\end{tabular} & 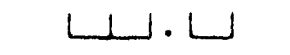 & لـL \\
\hline Wh. & 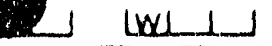 & $\square 2$ No & & & \\
\hline
\end{tabular}

omments:

Page 1 of 10 
BL. TORE COF'YING FORM, ATTACH STIE IDENTIFKCATHON LABEL

ORENTER.

Elk Hills Naval letroleum

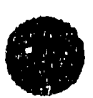

EPA $10 N($
Keserve Number I

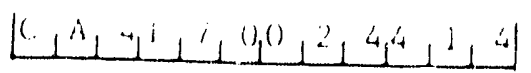

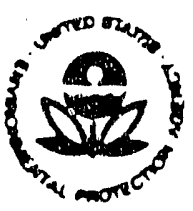

U.S. ENVIRONMENTAL

PROTECTION AGENCY

1989 Hazardous Waste Report

FORM

GM
WASTE GENERATION AND

MANAGEMENT

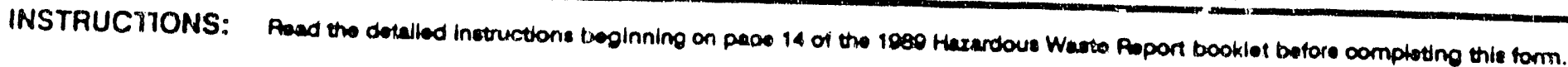

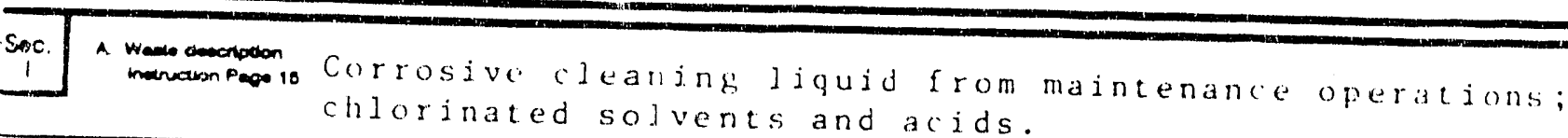

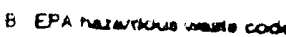

Poon 13

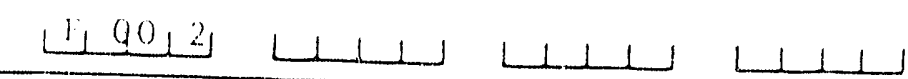

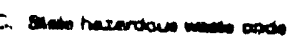

Prop 10

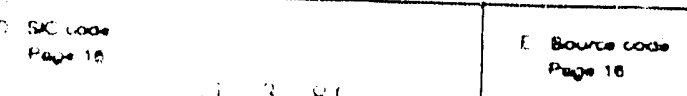

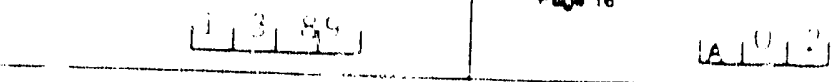

ite umeinimot

How 17

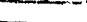

(1)

LI

Pno 1 1?

Form coose

Pow 10

L إن

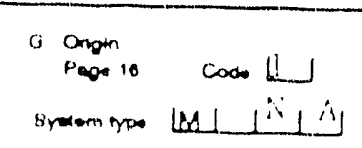

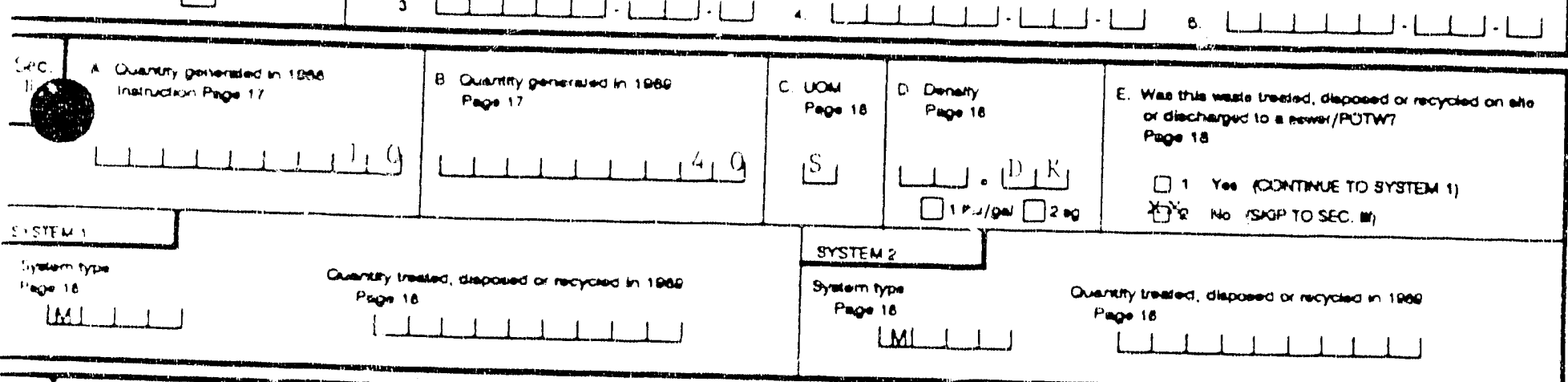

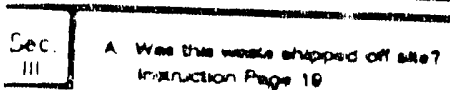

tii Ya RONTMUE TO BOX ET

D2 AN HSOP YO SEC.M

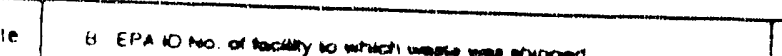

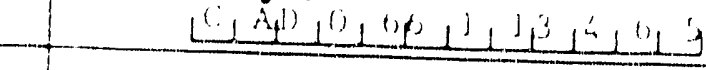

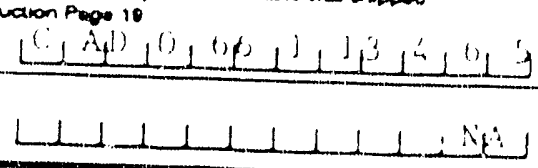

\begin{tabular}{|c|c|}
\hline C. Symem inpen & $\left.|\Delta|^{\prime \prime}\right)^{\prime}$, \\
\hline & $|B| \perp \mid$ \\
\hline
\end{tabular}

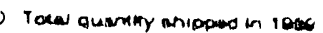
Puxpor 10 $L$

mom

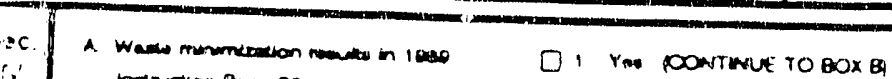

$\because$ Inotracon Pape 20

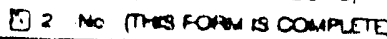
B Aarity
Paos 21
C. Othor Peop 21

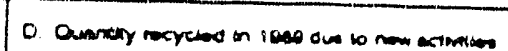 Pupos 21

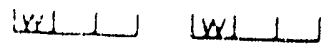
[,$~ r o o$
$1 \perp \perp 11 / 111:$ Peos 21
Papo $2 a$
E Mavipy/Produrtion indw.
F. Bowos Restuation Ouartify
لـ

i 


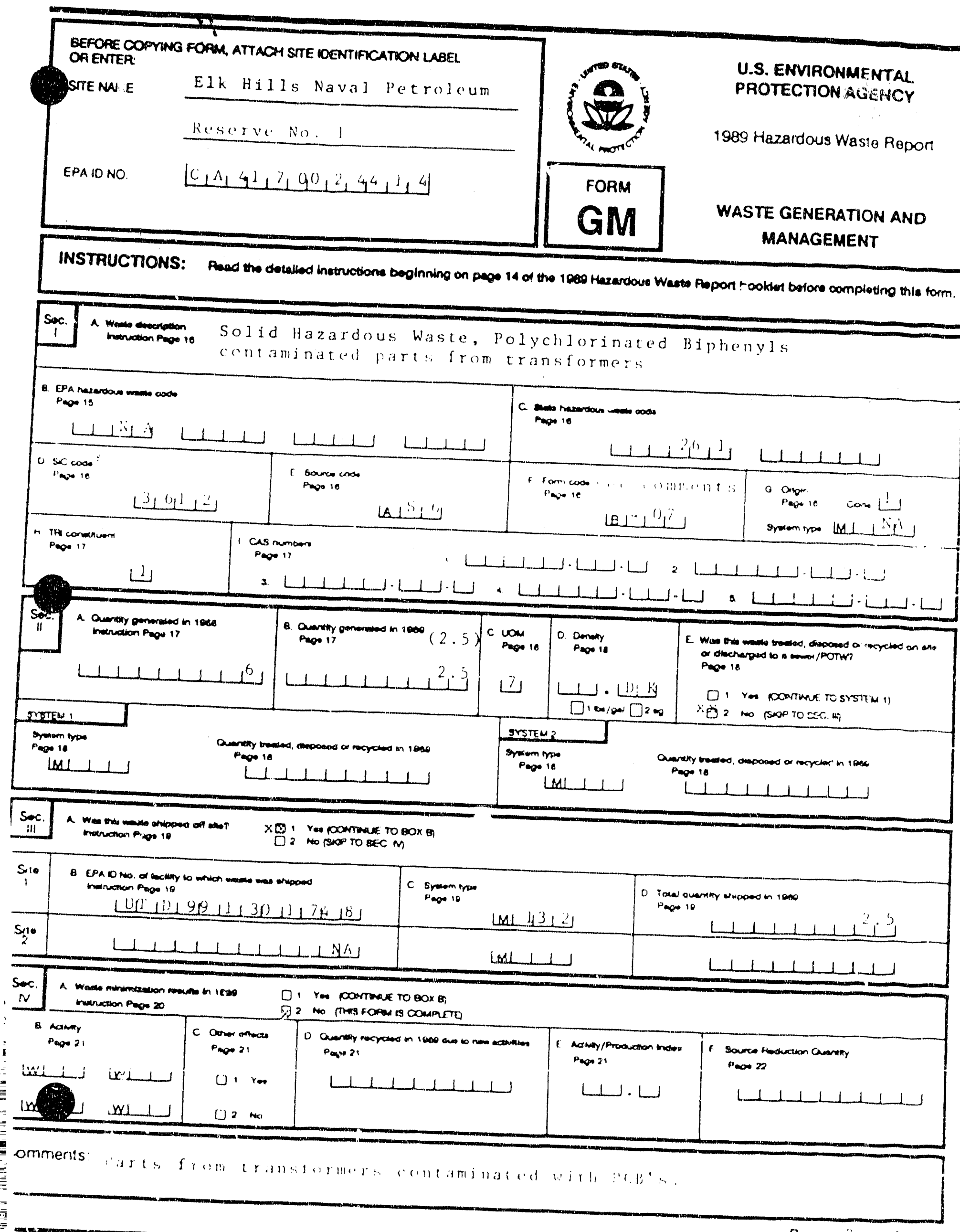




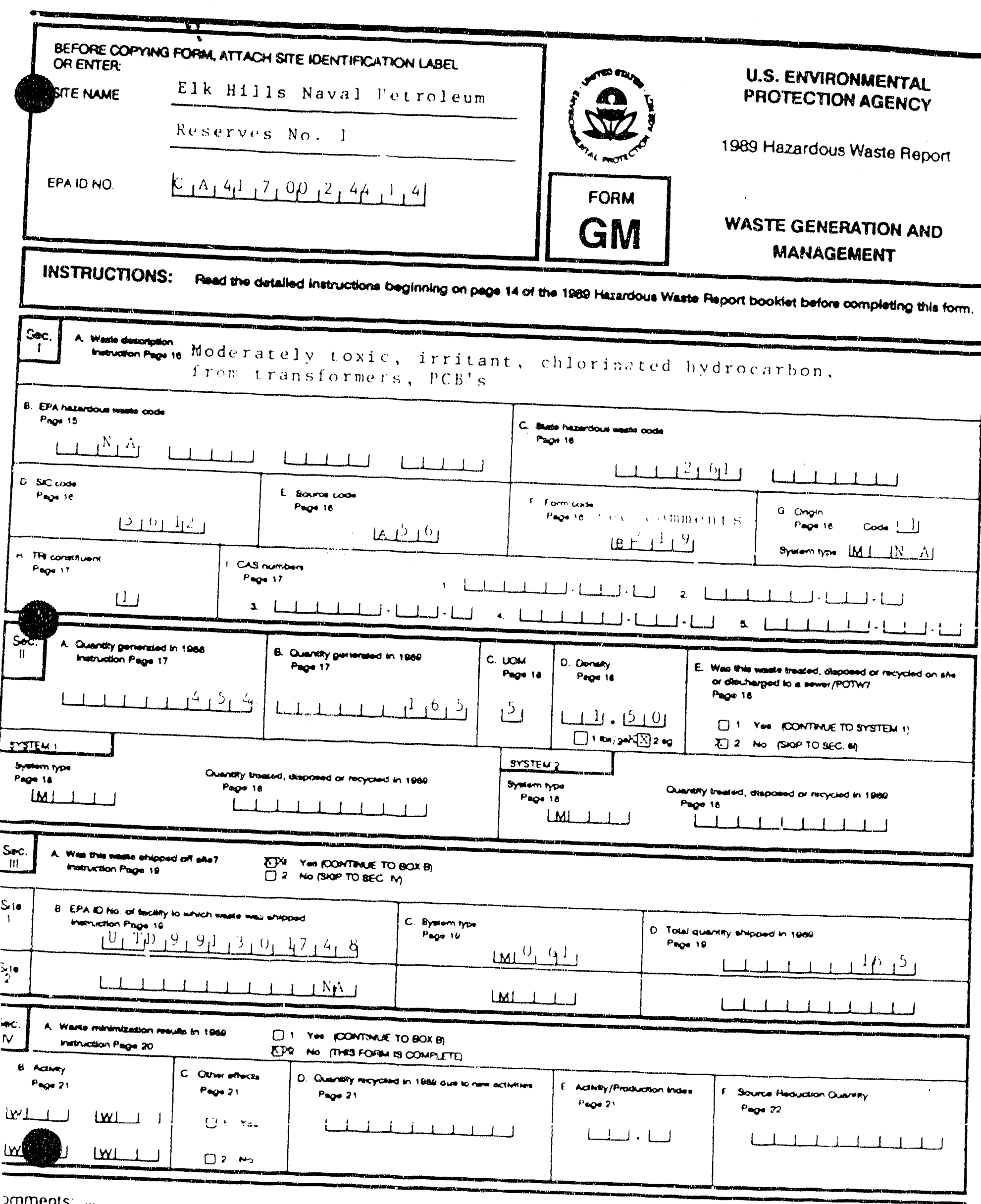

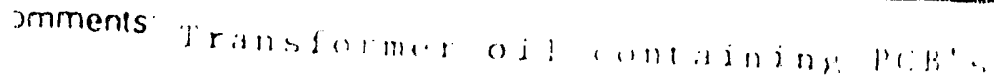




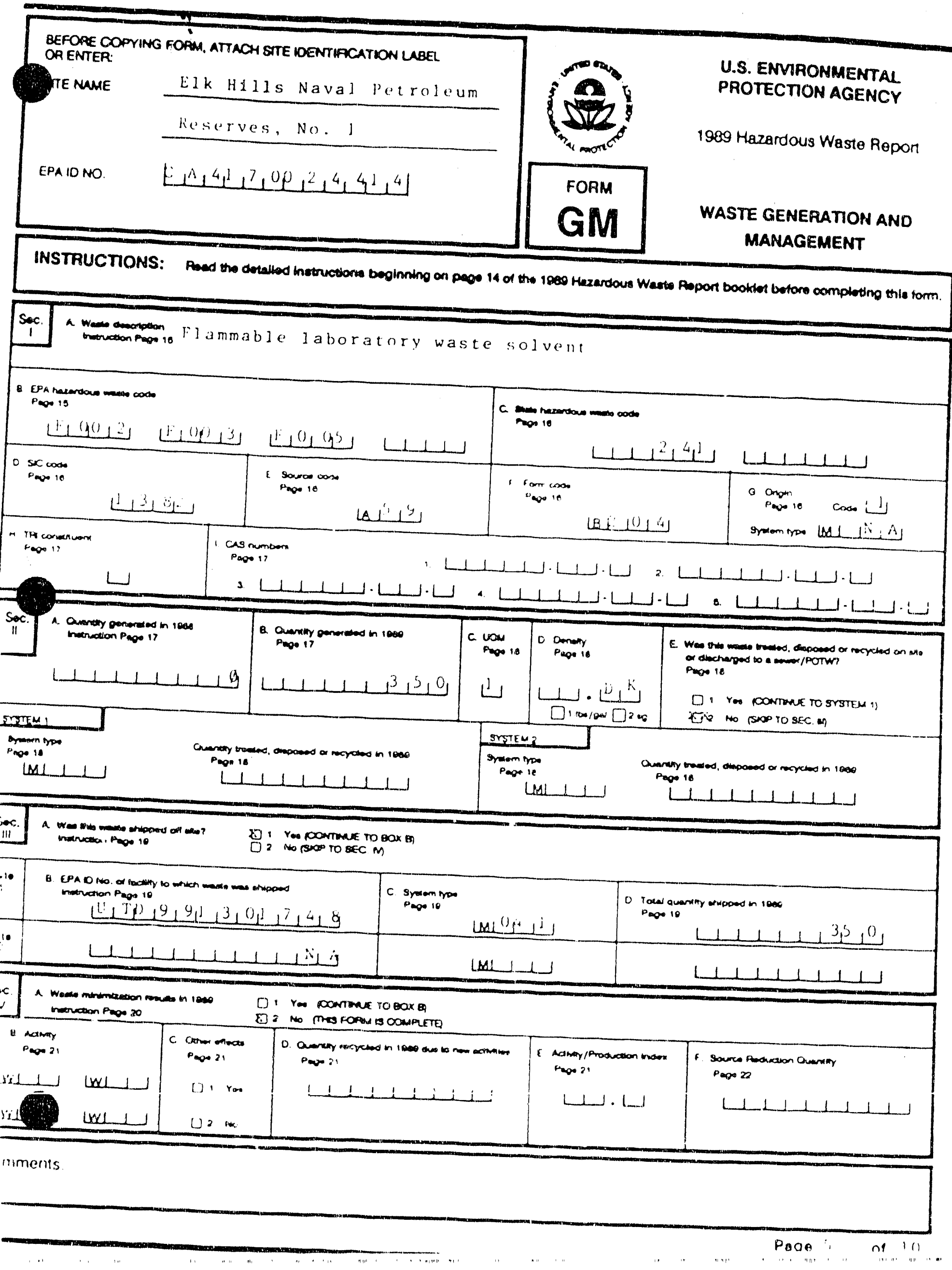




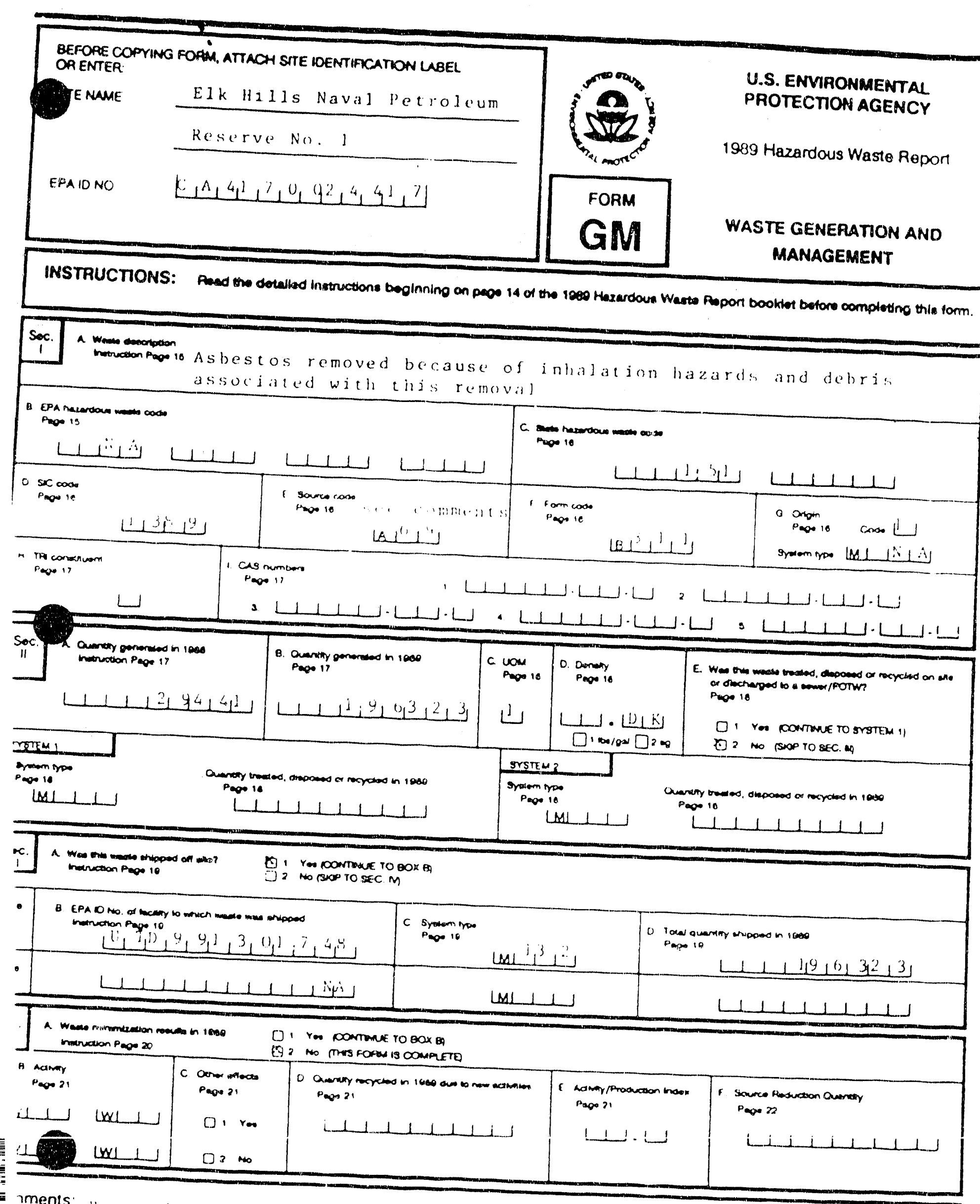

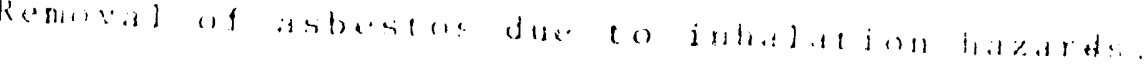




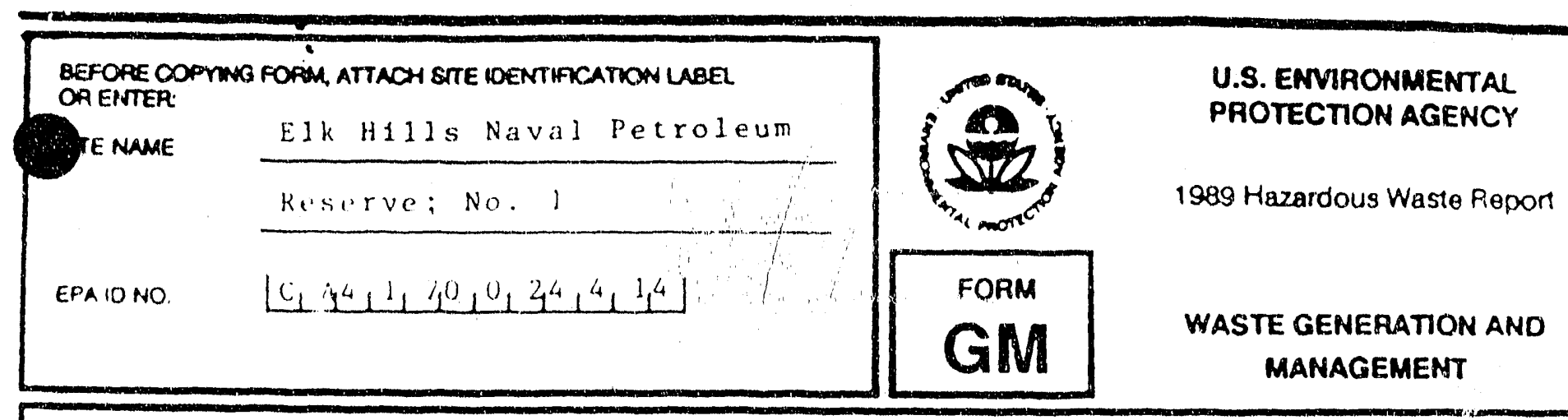

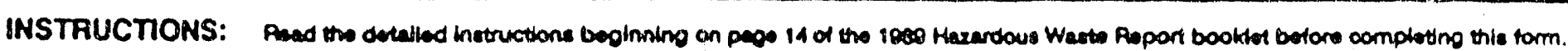

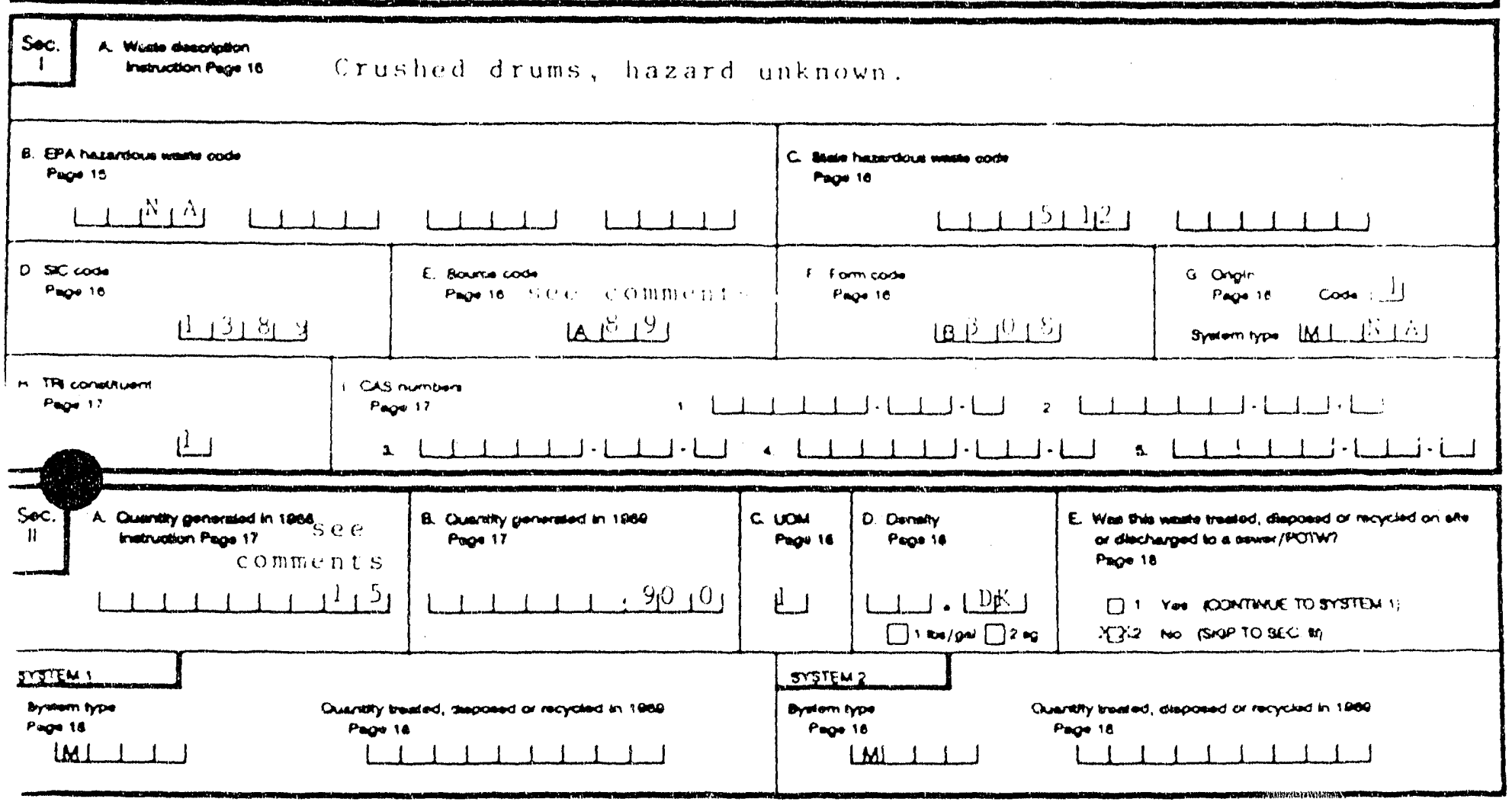

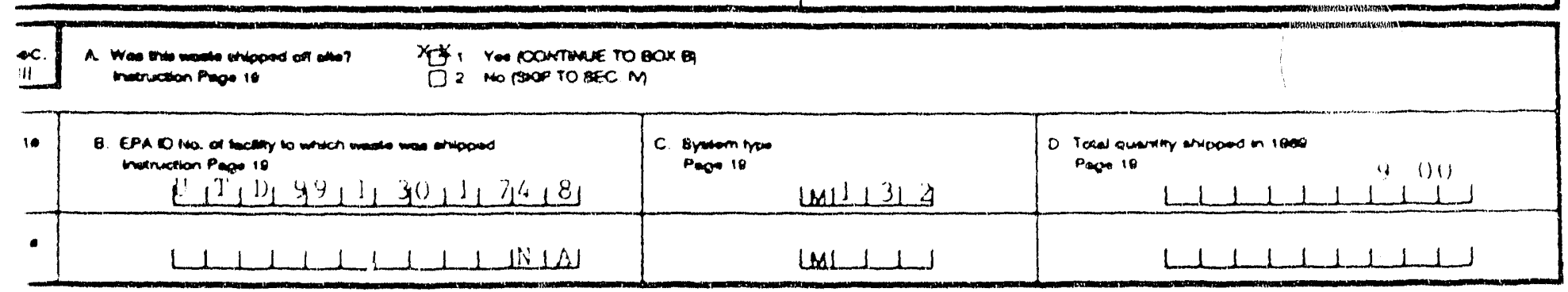

\begin{tabular}{|c|c|c|c|c|c|}
\hline a & tim Prasion 20 & $\infty$ in ion & 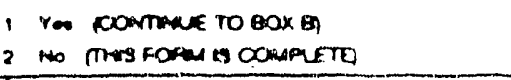 & & \\
\hline is nammer & & 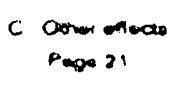 & 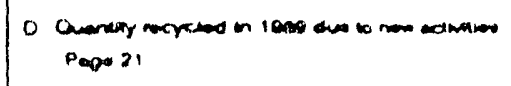 & 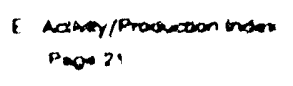 & 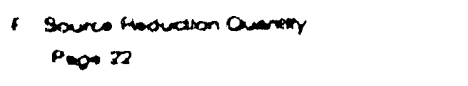 \\
\hline N & $|x|+\mid\rfloor$ & D. res & $|1| 1|1| 1 \mid 1$ & 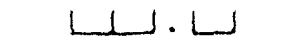 & 1 \\
\hline$x 1$ & $|x| \perp \mid$ & $\square=\infty$ & & & \\
\hline
\end{tabular}

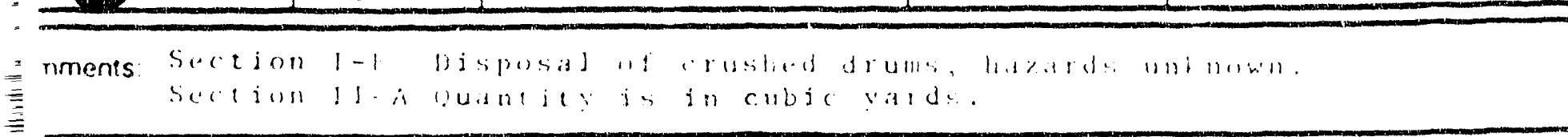




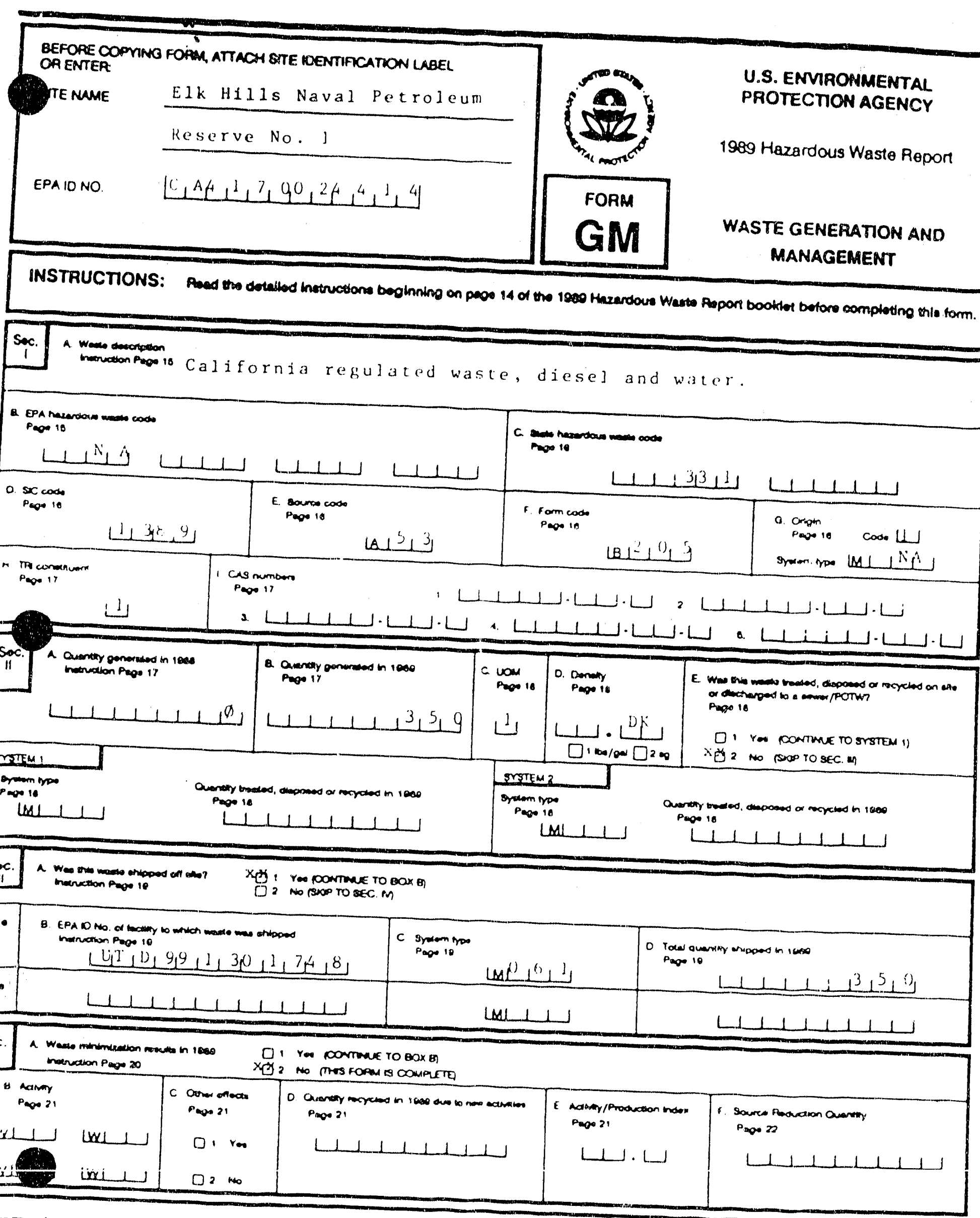

mments 


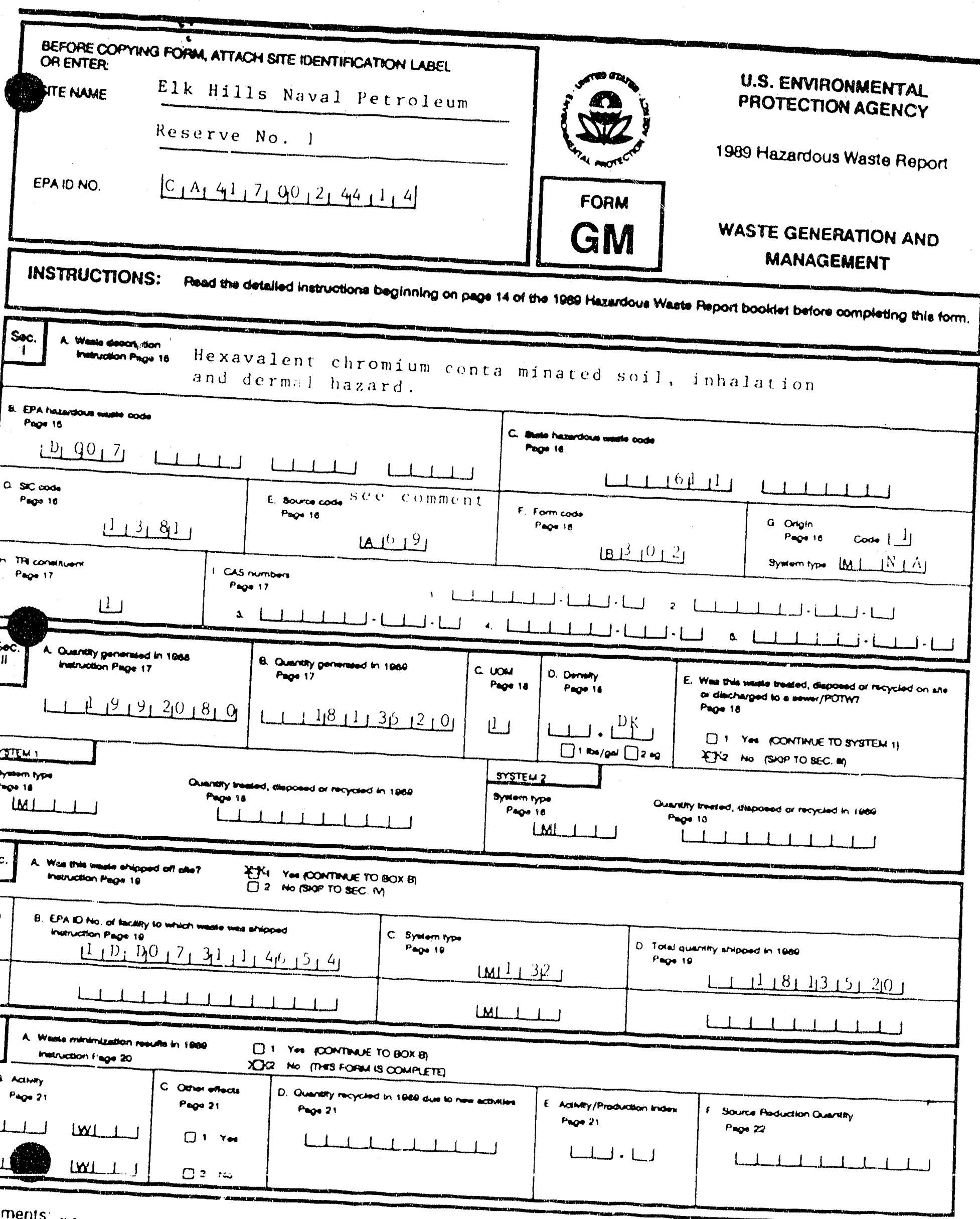

nments

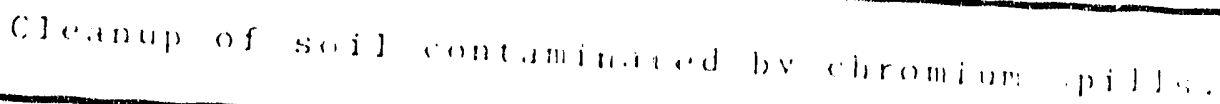




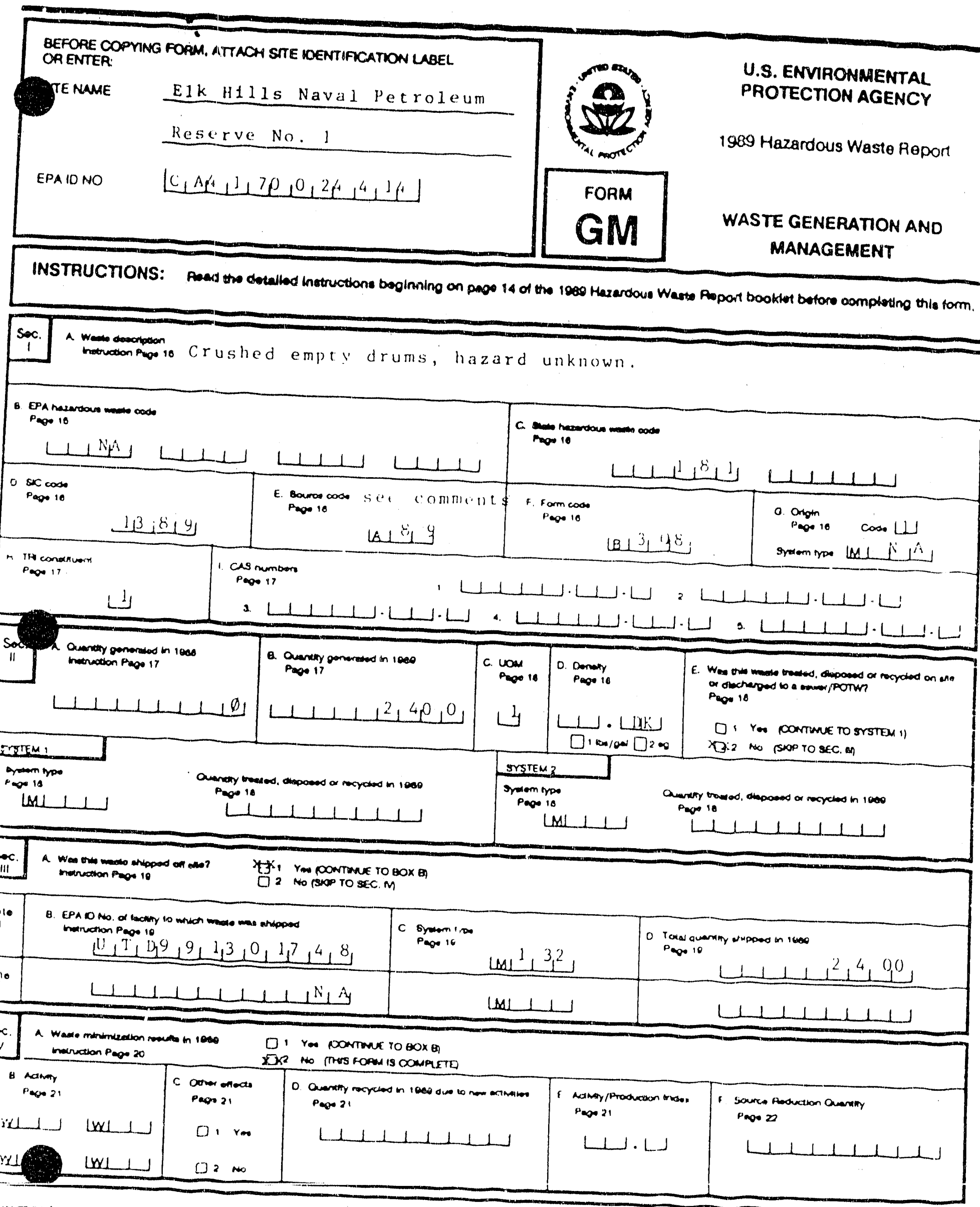

inments

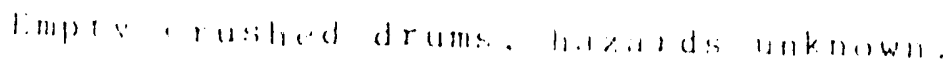

Page 10 of 10 


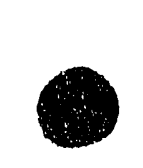

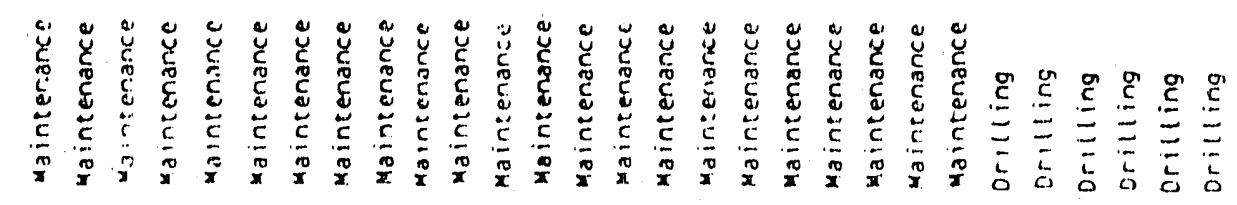

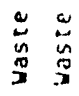

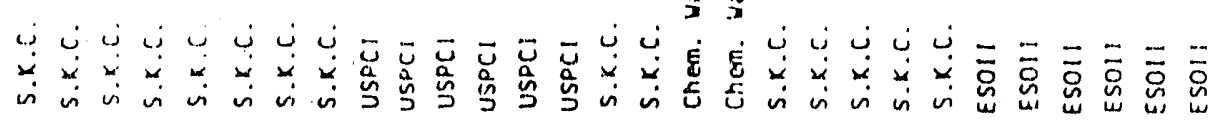

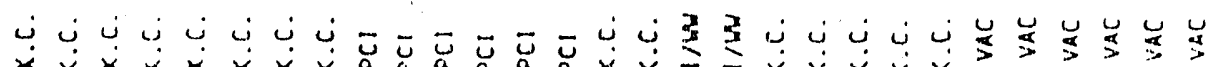

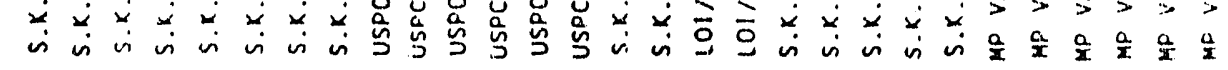

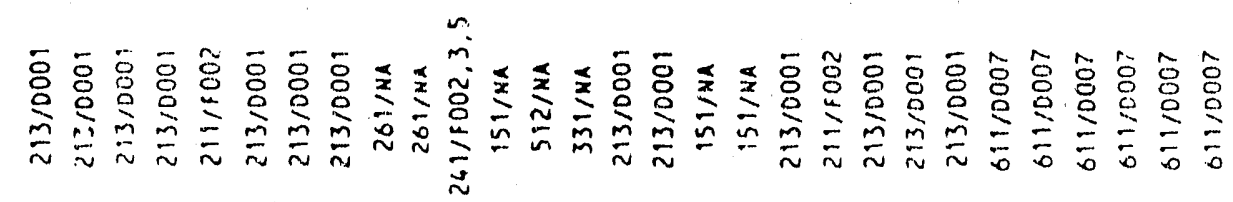

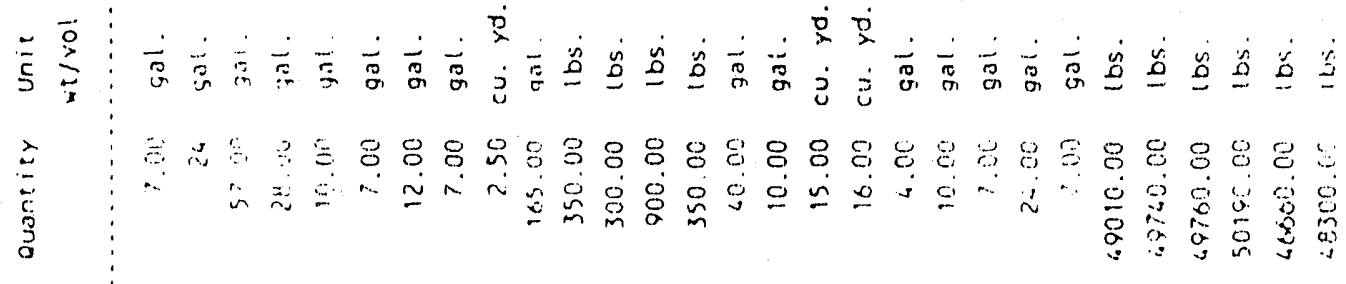

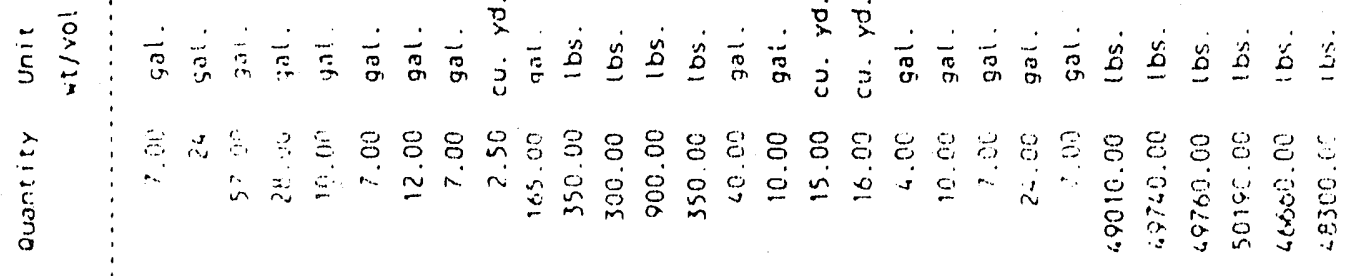

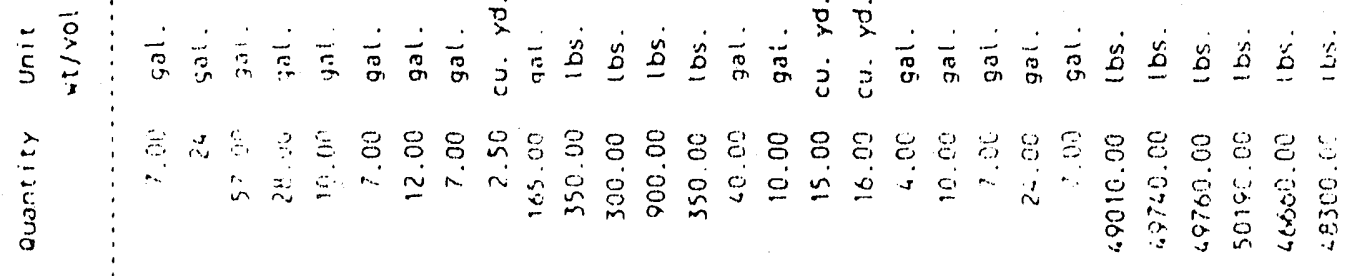

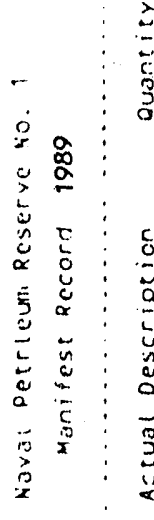

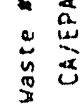

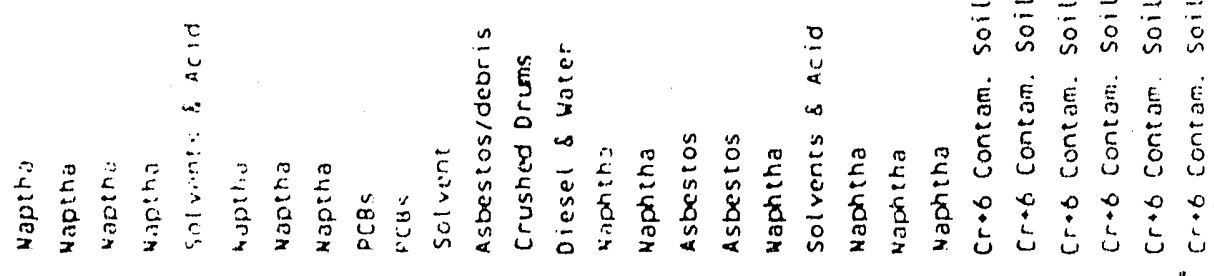

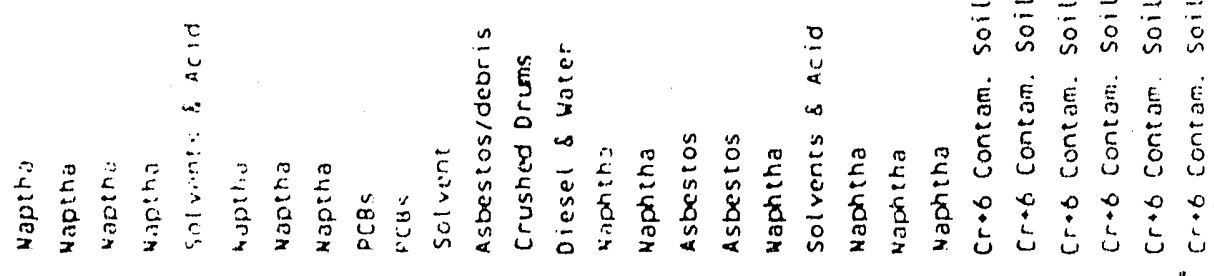

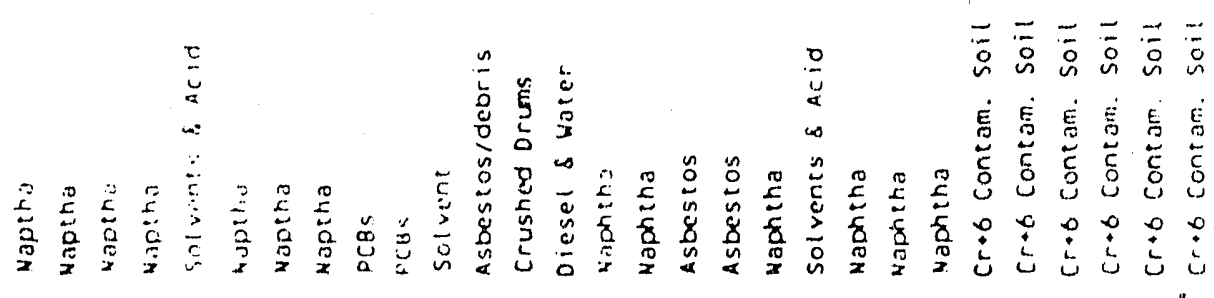

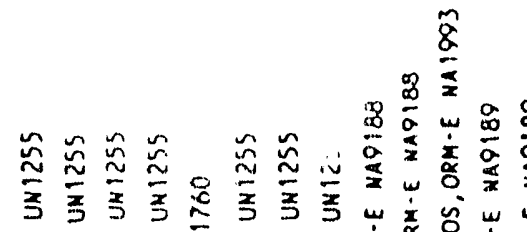

$\frac{5}{2}$

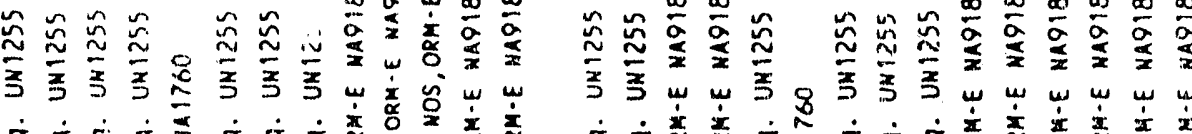

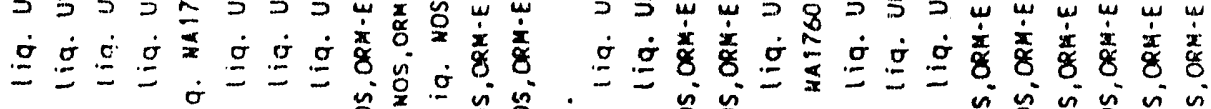

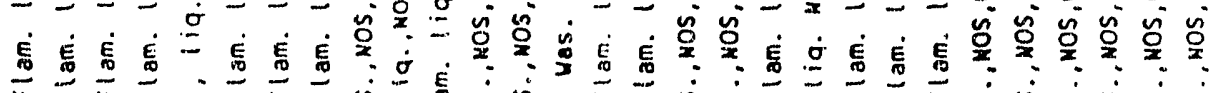

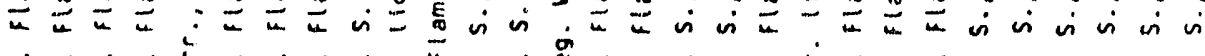

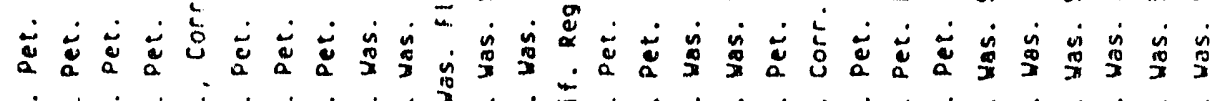

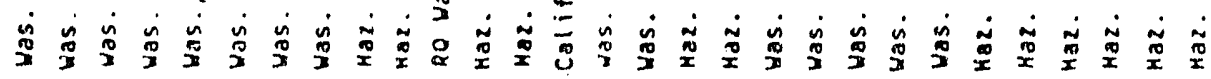

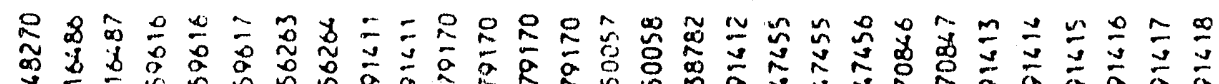

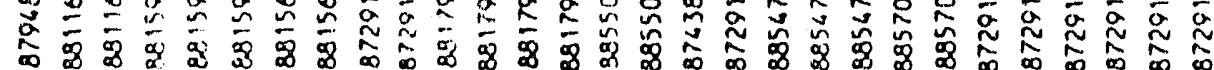

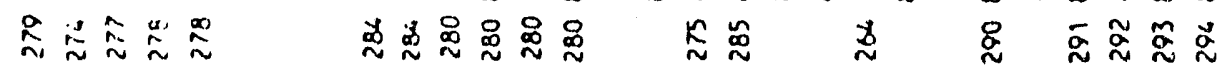

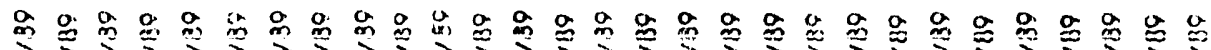
。 


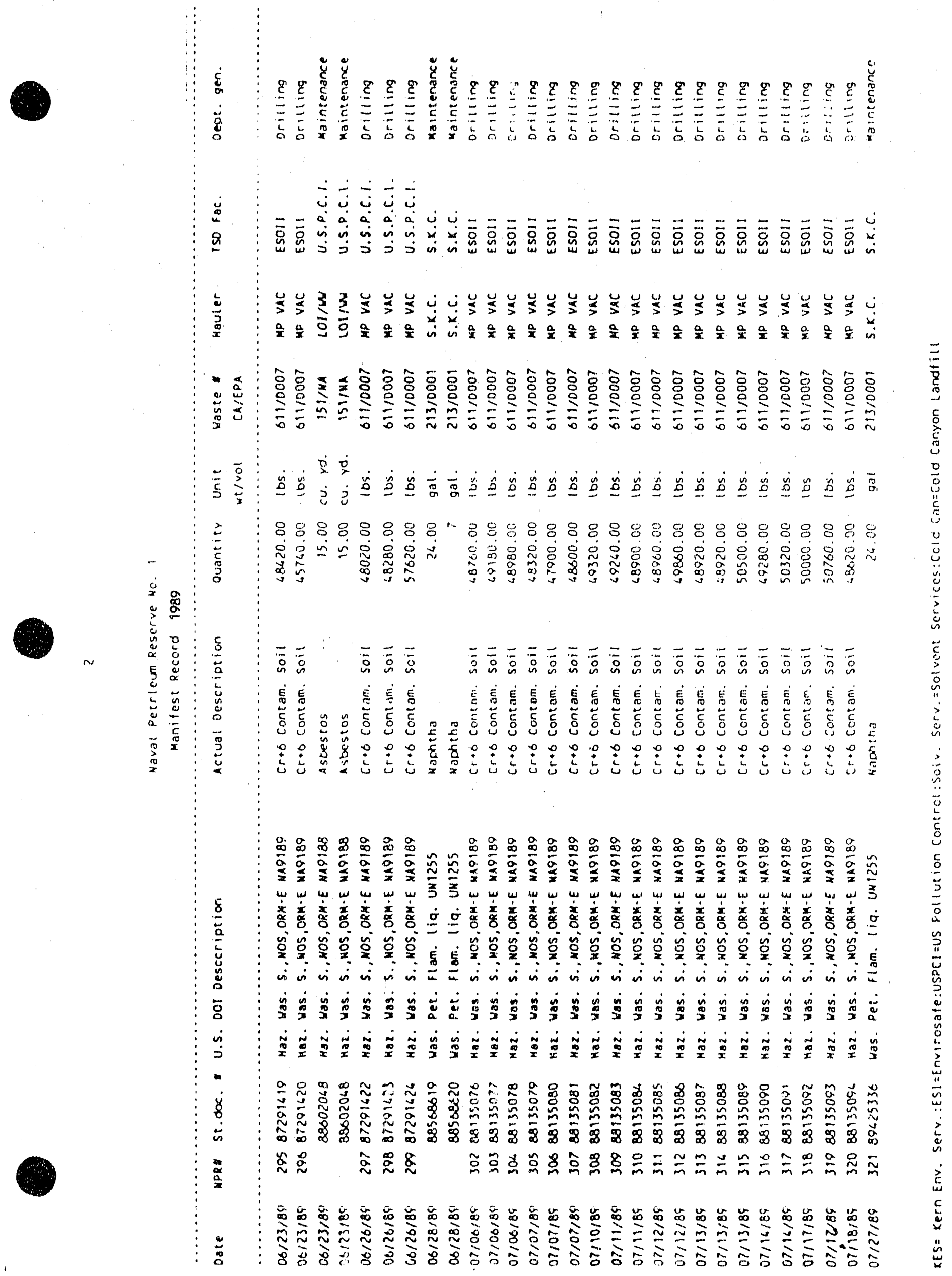


My

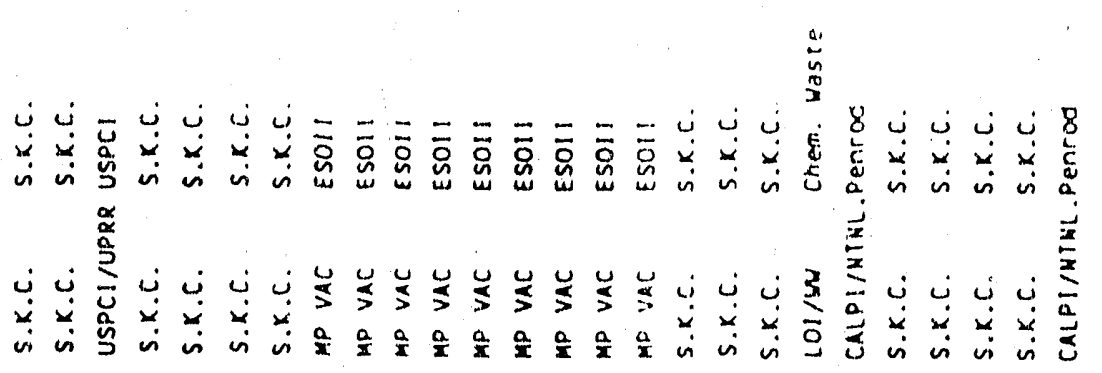

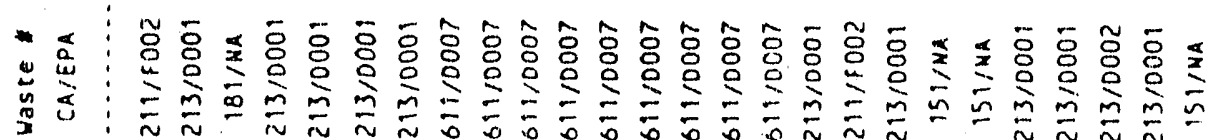

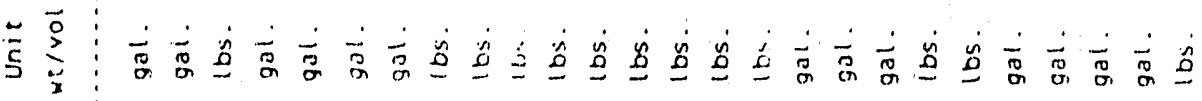

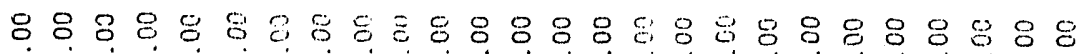

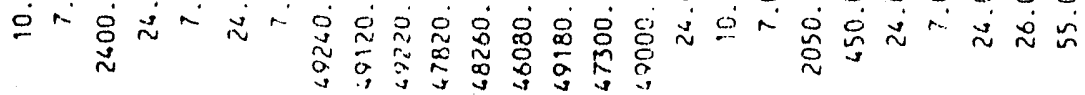

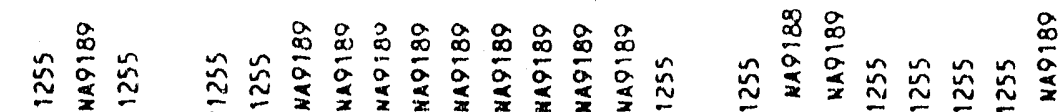

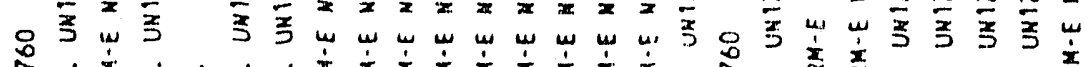

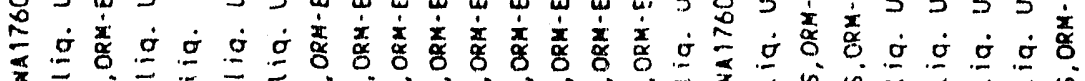

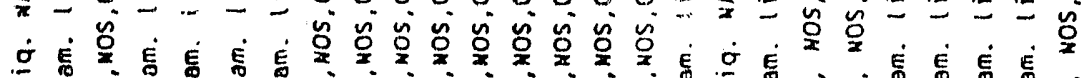

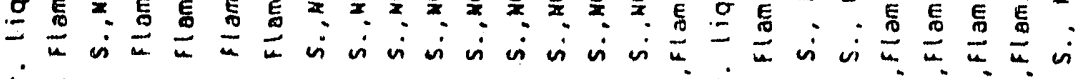

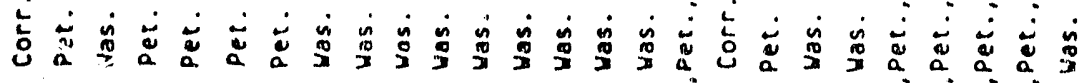

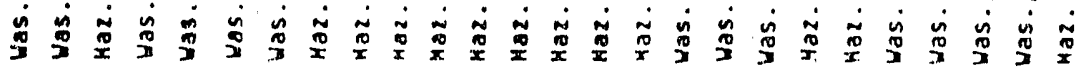

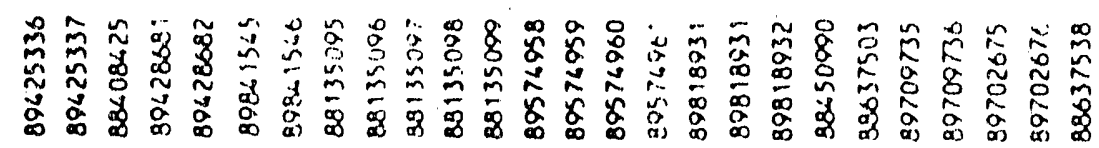

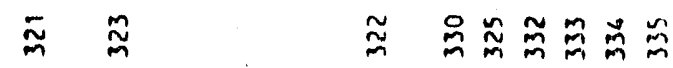

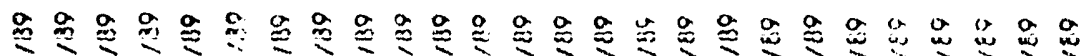

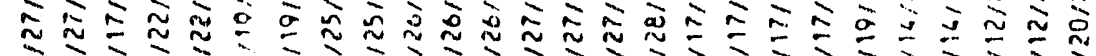

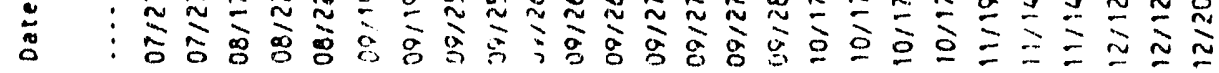




$$
\text { APPENDIX G }
$$

SUMMARY OF NPR-1 1989 CLASS II WELL DATA SUBMITTED

TO THE CALIFORNIA DIVISION OF OIL AND GAS 


\section{TABLE OF CONTENTS}

\section{APPENDIX G}

I. Summary of 1989 Mechanical Integrity Surveys for Class II Water Disposal Wells

II. Summary of 1989 Mechanical Integrity Surveys for Class II Waterflood Wells

III. Summary of Monthly Summaries of 1989 Class II Wells (Wastewater and Waterflood Wells) Data Reported to the California Division of Oil and Gas 
I. Summary of 1989 Mechanical Integrity Testing for Class II Water Disposal Wells

\begin{tabular}{|c|c|c|}
\hline Well/Sec. & Dates Surveyed & Comments \\
\hline 48WD-7G & $10 / 26 / 89$ & \\
\hline $68 \mathrm{WD}-7 \mathrm{G}$ & $08 / 15 / 89$ & \\
\hline 78WD-7G & $07 / 18 / 89$ & \\
\hline 88WD-7G & $10 / 05 / 89$ & \\
\hline 18WD-8G & $07 / 19 / 89$ & \\
\hline $56 \mathrm{WD} \cdot 10 \mathrm{G}$ & $04 / 85$ & No injection, Well not in use \\
\hline $61 \mathrm{WD}-18 \mathrm{G}$ & $04 / 06 / 89$ & \\
\hline $71 \mathrm{WD}-18 \mathrm{G}$ & 03/09/89,04/06/89 & \\
\hline $81 \mathrm{WD}-18 \mathrm{G}$ & $05 / 23 / 89$ & No injection, Well not in use \\
\hline $13 \mathrm{WD}-24 \mathrm{Z}$ & $01 / 06 / 89$ & \\
\hline $22 \mathrm{WD}-24 \mathrm{Z}$ & $09 / 12 / 89$ & \\
\hline $23 \mathrm{WD}-24 \mathrm{Z}$ & $09 / 12 / 89$ & \\
\hline 24WD-24Z & 09/20/89 & No injection, Well not in use \\
\hline 51WD-26Z & 03/09/89 & \\
\hline $42-16 \mathrm{G}$ & $02 / 16 / 89$ & \\
\hline $21-15 G$ & $05 / 23 / 89$ & No injection, Well not in use \\
\hline
\end{tabular}


II. Summary of 1989 Mechanical Integrity

Surveys for Class II Water Flood Wells 


\section{Summary of 1989 Mechanical Integrity Surveys for class II Water Flood Wells}

WELI./SEC

$313-26$

$324-26$

$333-20$

$352-20$

$353-20$

$371-26$

$314-30$

$315-3 G$

$324-3 G$

$335 \cdot 36$

$344 \cdot 36$

$354-36$

$364-3 G$

$384-30$

$315-40$

$326-40$

$335-4 G$

$346 / 40$

$355-40$

$364-40$

$365-4 Q$

$365-40$

$375-46$

$384-40$

313.56

$314 x-56$

$324 X-5 G$

$335 A-5 G$

$344-5 \mathrm{G}$

$355 \mathrm{X} \cdot 5 \mathrm{G}$

$366 \times-5 G$

$375-5 G$

386-5G

$342 A-6 G$

$363-6 \mathrm{G}$

$384-6 G$

$324 A-7 R$

$333 A-7 R$

$337 A-7 R$

$348 A-7 k$

$373 a-7 R$

$384 x-7 k$

$313-8 R$

$344-8 R$

$364-8 R$

375-8R

$313 A-17 k$

$333 A-17 R$

$375 x-17 R$

$354 x-17 R$

$386-17 R$

318- IOR

$351 A-18 R$

$3652 A-18 R$

$382 A-18 R$

$313 A-19 R$

$327-23 R$

$336 A-23 R$

$336 x-23 R$

$316 A-24 R$
DATES SURVEYED

$06 / 20 / 89$

$03 / 13 / 89$

$02 / 02 / 89$

05/05/89

$05 / 11 / 89$

05/05/89

$04 / 17 / 89$

$02 / 17 / 89$

$03 / 13 / 89$

$04 / 04 / 89$

$04 / 18 / 89$

$01 / 09 / 90$

$04 / 14 / 89$

$02 / 10 / 89$

$05 / 15 / 89$

$04 / 14 / 89$

$05 / 25 / 89$

$06 / 08 / 89$

$02 / 21 / 89$

$04 / 17 / 89$

$04 / 19 / 89$

$04 / 19 / 89$

$04 / 12 / 89$

$04 / 21 / 89$

$01 / 30 / 89$

$04 / 17 / 89$

$06 / 01 / 89$

$03 / 03 / 89$

$01 / 18 / 89$

$04 / 10 / 89$

05/04/89

03/07/89

$03 / 03 / 89$

$05 / 31 / 89$

$03 / 10 / 89$

$01 / 31 / 89$

$06 / 01 / 89$

$03 / 09 / 89$

$06 / 06 / 89$

n5/31/89

$12 / 13 / 88$ (LAST SURVEY)

03/09/89

$06 / 02 / 89$

$0.6 / 30 / 89$

$03 / 10 / 89$

$03 / 09 / 89$

$03 / 01 / 89$

03/08/89

$11 / 08 / 89$

$11 / 02 / 89$

$11 / 03 / 89$

$03 / 15 / 89$

$09 / 25 / 89$

$03 / 10 / 89$

$06 / 29 / 89$

$03 / 06 / 89$

$02 / 06 / 89$

$01 / 09 / 89$

$03 / 22 / 89$

$05,25 / 89$

$01 / 19 / 89$

$09 / 14 / 89$

$10 / 20 / 89$

$05 / 30 / 89$

$09 / 13 / 89$
COMENTS

NO INJECTION UNTIL 12/88

AMOUNT OF INJECTION MINIMAL

CAN'T SURVEY-LON RATE

AMOUNT OF IMAECTION MIMIMAL.
$09 / 26 / 89 \quad 11 / 28 / 89$ 


$$
\begin{aligned}
& 326-216 R \\
& 337 x-24 R \\
& 348 \mathrm{X}-24 \mathrm{R} \\
& 318-25 R \\
& 361 x-25 R \\
& 3 T 1 X-25 R \\
& 311-26 R \\
& 322 A-26 R \\
& 334 X-26 R \\
& 344 A-26 R \\
& 356 X-26 R \\
& \text { 367-26R } \\
& 378-26 R \\
& 378 X-26 R \\
& 321 x-36 R \\
& 332 x-36 R \\
& 343 x-36 R \\
& 355 x-36 R \\
& 366 \mathrm{X}-36 \mathrm{R} \\
& \text { 376-36R } \\
& \text { 387-36R } \\
& 318 \mathrm{~L}-29 \mathrm{~S} \\
& 313 x-305 \\
& \text { 323-305 } \\
& 324 \times \mathrm{L}-30 \mathrm{~S} \\
& 345 x-305 \\
& 365-30 \text { S } \\
& \text { 377-30S } \\
& \text { 32BA-315 } \\
& \text { 331-325 } \\
& \text { 333-325 } \\
& 351 x-325 \\
& \text { 353-325 } \\
& 373 \cdot 325 \\
& \text { 382-32s } \\
& 314-335 \\
& \text { 333-335 } \\
& \text { 335-33s } \\
& 353-335 \\
& \text { 355-33s } \\
& 363 x-335 \\
& 375 \cdot 335 \\
& 382 \text {-335 } \\
& \text { 315-345 } \\
& \text { 322.34S } \\
& 324-345 \\
& \text { 326-34S } \\
& 335-34 \mathrm{~S} \\
& 342-345 \\
& \text { 344-34S } \\
& 355-345 \\
& 362-345 \\
& \text { 373-34S } \\
& 375.34 \mathrm{~S} \\
& 313 \text {-35s } \\
& 322-35 s \\
& 342 x-355 \\
& 363 x-355 \\
& \text { 364-355 } \\
& \text { 368-35s } \\
& 374 x-355 \\
& \text { 375-35s } \\
& \text { 377-35s } \\
& 388 x-35 s \\
& 316 x-365 \\
& 327 x-132 \\
& 347 A-132
\end{aligned}
$$

02/28/89

$04 / 19 / 89$

03/03/89

$04 / 21 / 89$

$06 / 13 / 89$

$01 / 25 / 89$

$06 / 01 / 89$

$04 / 21 / 89$

$01 / 25 / 89$

$12 / 01 / 89$

$03 / 11 / 89$

$06 / 12 / 89$

03/10/89

$03 / 01 / 89$

03/03/89

$02 / 01 / 89$

$04 / 01 / 89$

02/21/89

$02 / 02 / 89$

$01 / 31 / 89$

$06 / 19 / 89$

$01 / 19 / 89$

03/06/89

$03 / 01 / 89$

02/02/89

$12 / 01 / 89$

$12 / 01 / 89$

$08 / 07 / 89$

05/30/B9

$04 / 03 / 89$

$01 / 18 / 89$

$02 / 14 / 89$

$01 / 01 / 89$

$05 / 20 / 89$

$04 / 21 / 89$

05/15/89

$02 / 09 / 89$

05/26/89

$12 / 01 / 89$

03/06/89

$02 / 14 / 89$

$03 / 06 / 89 \quad 04 / 2.1 / 89$

$01 / 03 / 89 \quad 07 / 05 / 89$

$02 / 28 / 89$

$02 / 13 / 89$

$02 / 01 / 89$

$05 / 20 / 89$

$04 / 18 / 89$

$01 / 12 / 89$

$06 / 05 / 89$

$02 / 15 / 89$

$02 / 15 / 89$

$03 / 07 / 89$

$03 / 23 / 89$

$05 / 26 / 89$

$06 / 19 / 89$

$09 / 26 / 89 \quad 11 / 30 / 89$

$05 / 12 / 89 \quad 06 / 05 / 89 \quad 08 / 29 / 89$

08/07/89

$03 / 14 / 89$
NO INJECTION UNTIL $1 / 90$

NO INJECTION-WELL NOT IN USE

SHUT - IN EQUALIZED

NEW WELL (INITIAL SURVEY)

SHUT - IN EQUALIZED

NEW WELL (INITIAL SURVEY)

NEW WELL (INITIAL SURVEY)

AMOUNT OF INJECTION MINIMAL.

SHUT - IN EQUALIZED

NEW WELL (INITIAL SURVEY)

NO IAJECTION-HELL NO IN USE

04/03/89 HEW WELL (INITIAL SURVEY 3/23) 


$\begin{array}{lllll}367-132 & 01 / 17 / 89 & 09 / 15 / 89 & \\ 386-132 & 02 / 03 / 89 & 09 / 25 / 89 & \\ 315-242 & 02 / 03 / 89 & 09 / 12 / 89 & & \\ 317-242 & 03 / 20 / 89 & & & \\ 326-242 & 01 / 16 / 89 & 02 / 02 / 89 & 03 / 20 / 89 & 11 / 29 / 89 \\ 337-242 & 01 / 16 / 89 & 11 / 29 / 89 & & \\ 337 A-242 & 01 / 17 / 89 & 11 / 13 / 89 & 11 / 28 / 89 & \\ 338-242 & 03 / 20 / 89 & & & \\ 348 \times-242 & 07 / 12 / 89 & & \\ 391-242 & & & & \end{array}$

NO INJECTION-WELL NOT IN USE 
III. Summary of Monthly Summaries of 1989 Class II

Wells (Wastewater Injection Wells and Waterflood

Wells) Data Reported to the California

Division of Oil and Gas 
III. MaVAl PETROLEUM Reserve no. 1 WATER IAJECTION SUMUARIES 1989

\begin{tabular}{|c|c|c|c|c|c|}
\hline WELL/SECT & YR & MONTH & $\begin{array}{l}\text { DAYS ON } \\
\text { IMJECTION }\end{array}$ & $\begin{array}{l}\text { MATER } \\
\text { INJECTION }\end{array}$ & $\begin{array}{l}\text { TUBING } \\
\text { PRESSURE }\end{array}$ \\
\hline $484 D-7 G$ & 89 & 1 & 29 & -351775 & 160 \\
\hline $4840-7 c$ & 89 & 2 & 28 & -344648 & 153 \\
\hline $4840-76$ & 89 & 3 & 31 & -400227 & 169 \\
\hline $4840-7 G$ & 89 & 4 & 30 & -407800 & 176 \\
\hline $48100-76$ & 89 & 5 & 31 & -389810 & 169 \\
\hline $4840-7 c$ & 89 & 6 & 30 & -460363 & 120 \\
\hline $4840-7 G$ & 89 & 7 & 31 & -494561 & 129 \\
\hline $48 \mathrm{WD}-7 \mathrm{G}$ & 89 & 8 & 31 & -494733 & 129 \\
\hline $4840-7 G$ & 89 & 9 & 30 & -483083 & 140 \\
\hline $4840-76$ & 89 & 10 & 31 & -481883 & 137 \\
\hline $4840-76$ & 89 & 11 & 30 & -420135 & 128 \\
\hline $4840-7 G$ & 89 & 12 & 31 & -376787 & 113 \\
\hline $6840-7 G$ & 89 & 1 & 29 & -260499 & 126 \\
\hline $6810-76$ & 89 & 2 & 28 & -300563 & 96 \\
\hline $6840-7 c$ & 89 & 3 & 31 & -358273 & 110 \\
\hline $684 D-7 G$ & 89 & 4 & 30 & -3399164 & 128 \\
\hline $681 \mathrm{MD}-7 \mathrm{G}$ & 89 & 5 & 31 & -326218 & 126 \\
\hline $6840-7 G$ & 89 & 6 & 30 & -272605 & 80 \\
\hline $68100-7 G$ & 89 & 7 & 29 & -153758 & 30 \\
\hline $6810-7 G$ & 89 & 8 & 31 & -231633 & 32 \\
\hline $6840-76$ & 89 & 9 & 30 & -210061 & 28 \\
\hline $6840-76$ & 89 & 10 & 31 & -202376 & 31 \\
\hline $6840-76$ & 89 & 11 & 30 & -222095 & 34 \\
\hline $6840-76$ & 89 & 12. & 31 & -233023 & 31 \\
\hline $7840-76$ & 89 & 1 & 31 & -62761 & 157 \\
\hline $7840-70$ & 89 & 2 & 28 & -51678 & 151 \\
\hline 7BLD-7G & 89 & 3 & 31 & -58755 & 164 \\
\hline $7810-76$ & 89 & 4 & 30 & -44738 & 173 \\
\hline $7840-7 G$ & 89 & 5 & 31 & -43262 & 168 \\
\hline $78-5-76$ & 89 & 6 & 27 & -53119 & 136 \\
\hline $78 w 0-76$ & 89 & 7 & $3 i$ & -88741 & 43 \\
\hline $7800-7 G$ & 89 & 8 & 31 & -52402 & 51 \\
\hline $7860-7 G$ & 89 & 9 & 30 & -41022 & 61 \\
\hline $78 W 0-7 G$ & 89 & 10 & 31 & -54668 & 63 \\
\hline $7840-7 G$ & 89 & 11 & 30 & -44058 & 61 \\
\hline $7840-7 G$ & 89 & 12 & 31 & -46902 & $\pi$ \\
\hline $8840-76$ & 89 & 1 & 31 & -234807 & 66 \\
\hline $8840-76$ & 89 & 2 & 28 & -165720 & 58 \\
\hline 88WD-7G & 89 & 3 & 31 & -216694 & 87 \\
\hline $8840-7 G$ & 89 & 4 & 29 & -206780 & 88 \\
\hline $8840-7 \mathrm{G}$ & 89 & 5 & 30 & -238194 & 83 \\
\hline $8810-7 c$ & 89 & 6 & 30 & -297852 & 98 \\
\hline $8 B D-7 G$ & 89 & 7 & 31 & -416592 & 144 \\
\hline $8840-70$ & 80 & 8 & 31 & -393112 & 158 \\
\hline $8840-76$ & 89 & 9 & 30 & -517262 & 176 \\
\hline $8810-7 G$ & 89 & 10 & 31 & -517689 & 187 \\
\hline
\end{tabular}


111. MAVAl PETROLEUM RESERVE NO. 1

WATER INJECTION SUMMARIES 1989

\begin{tabular}{|c|c|c|c|c|c|}
\hline WELL/SECT & YR & MONTH & $\begin{array}{l}\text { DAYS ON } \\
\text { IHJECTION }\end{array}$ & $\begin{array}{l}\text { WATER } \\
\text { INJECTION }\end{array}$ & $\begin{array}{l}\text { TUBING } \\
\text { PRESSURE }\end{array}$ \\
\hline & 80 & 19 & 80 & & 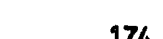 \\
\hline $\begin{array}{l}8640-76 \\
8240-76\end{array}$ & $\begin{array}{l}84 \\
89\end{array}$ & $\begin{array}{l}11 \\
12\end{array}$ & $\begin{array}{l}30 \\
29\end{array}$ & $\begin{array}{l}-430521 \\
-470234\end{array}$ & $\begin{array}{l}174 \\
147\end{array}$ \\
\hline $180-86$ & 89 & 9 & 20 & -5955 & 146 \\
\hline $1890-86$ & 89 & 2 & 28 & -6794 & 143 \\
\hline $1840-86$ & 89 & 3 & 29 & -5509 & 145 \\
\hline $18 \times 0-86$ & 89 & 4 & 26 & -3949 & 156 \\
\hline $1810-86$ & 89 & 5 & 31 & -12340 & 163 \\
\hline $1840-86$ & 89 & 6 & 30 & -7922 & 155 \\
\hline $1840-86$ & 89 & 7 & 39 & -24210 & 1314 \\
\hline $1840-86$ & 89 & 8 & 31 & -18541 & 158 \\
\hline $1840-86$ & 89 & 9 & 30 & -13717 & 158 \\
\hline $1840-86$ & 89 & 10 & 31 & -20912 & 155 \\
\hline $1840-86$ & 89 & 11 & 30 & -22051 & 150 \\
\hline $1840-8 G$ & 89 & 12 & 31 & -22776 & 143 \\
\hline $5610-10 G$ & 89 & 1 & 0 & 0 & 160 \\
\hline $5610-10 G$ & 89 & 2 & 0 & 0 & 160 \\
\hline 5610-10G & 89 & 3 & 0 & 0 & 160 \\
\hline 5610-10G & 89 & 4 & 0 & 0 & 160 \\
\hline $5610-106$ & 89 & 5 & 0 & 0 & 160 \\
\hline $5640-106$ & 89 & 6 & 0 & 0 & 160 \\
\hline $5610-10 G$ & 89 & 7 & 0 & 0 & 160 \\
\hline $5610-10 G$ & 89 & 8 & 0 & 0 & 160 \\
\hline $56+10-10 G$ & 89 & 9 & 0 & 0 & 160 \\
\hline $5610-106$ & 89 & 10 & 0 & 0 & 160 \\
\hline $5610-906$ & 89 & 11 & 0 & 0 & 160 \\
\hline $5640-10 G$ & 89 & 12 & 0 & 0 & 160 \\
\hline $6110-18 G$ & 89 & 1 & 31 & -546131 & 145 \\
\hline $6110-186$ & 89 & 2 & 28 & -445698 & 143 \\
\hline $6160-186$ & 89 & 3 & 31 & -576730 & 153 \\
\hline $6140-18 G$ & 89 & 4 & 30 & -610688 & 164 \\
\hline $61100-186$ & 89 & 5 & 34 & -572962 & 153 \\
\hline $6910-186$ & 89 & 6 & 30 & -500831 & 101 \\
\hline $6140-186$ & 89 & 7 & 29 & -536363 & 89 \\
\hline $61 W 0-18 G$ & 89 & 8 & 31 & -588092 & 113 \\
\hline $6110-186$ & 89 & 9 & 30 & -559939 & 132 \\
\hline $6160-180$ & 89 & 10 & 31 & -601642 & 140 \\
\hline $6160-186$ & 89 & 11 & 30 & -517759 & 142 \\
\hline $6110-18 c$ & 89 & 12 & 31 & -500730 & 142 \\
\hline $7910-986$ & 89 & 1 & 31 & -328869 & 125 \\
\hline $71 W D-180$ & 89 & 2 & 28 & -270528 & 123 \\
\hline $7100-186$ & 89 & 3 & 31 & -340183 & 142 \\
\hline $7140-180$ & 89 & 4 & 30 & $-34 \pi 778$ & 159 \\
\hline $7140-186$ & 89 & 5 & 31 & -337203 & 147 \\
\hline $7140-180$ & 89 & 6 & 30 & -228679 & 113 \\
\hline $7110-186$ & 89 & 7 & 30 & -130409 & 57 \\
\hline $7160-180$ & 89 & 8 & 31 & -164422 & 60 \\
\hline
\end{tabular}


11I. NAVAL PETROLEUM RESERVE NO. 1 MATER INJECTION SLMMARIES 1989

\begin{tabular}{|c|c|c|c|c|c|}
\hline WELL/SEC? & YR & MONTH & $\begin{array}{l}\text { DAYS OW } \\
\text { INJECTIOW }\end{array}$ & $\begin{array}{l}\text { WATER } \\
\text { INJECTION }\end{array}$ & $\begin{array}{l}\text { TUBING } \\
\text { PRESSURE }\end{array}$ \\
\hline $71100-180$ & 89 & 9 & 30 & -173718 & 62 \\
\hline $7910-186$ & 89 & 10 & 31 & -188325 & 60 \\
\hline $71100-186$ & 89 & 11 & 30 & $-1834,99$ & 57 \\
\hline $7160-186$ & 89 & 12 & 31 & -168665 & 54 \\
\hline $8140-186$ & 89 & 1 & 31 & -71282 & 62 \\
\hline $8110-18 G$ & 89 & 2 & 28 & -45341 & 62 \\
\hline 81LD-186 & 89 & 3 & 31 & -61920 & 84 \\
\hline $8160-180$ & 89 & 4 & 30 & -57412 & 84 \\
\hline $8140-180$ & 89 & 5 & 27 & -193778 & 88 \\
\hline $8110-180$ & 89 & 6 & 30 & -403239 & 131 \\
\hline $8140-186$ & 89 & 7 & 31 & -382913 & 134 \\
\hline $8960-186$ & 89 & 8 & 31 & -354826 & 146 \\
\hline $8160-186$ & 89 & 9 & 30 & -348243 & 149 \\
\hline $81100-186$ & 89 & 10 & 31 & -407574 & 144 \\
\hline $81160-180$ & 89 & 11 & 30 & $-4 \pi 7774$ & 133 \\
\hline $8160-186$ & 89 & 12 & 31 & -531759 & 102 \\
\hline $1310-242$ & 89 & 1 & 31 & -276837 & 181 \\
\hline $1340-242$ & 89 & 2 & 28 & -151173 & 149 \\
\hline $1310-242$ & 89 & 3 & 31 & -160007 & 139 \\
\hline $1340-242$ & 89 & 4 & 30 & -161174 & 146 \\
\hline $1310-242$ & 89 & 5 & 30 & -230829 & 128 \\
\hline $1340-24,2$ & 89 & 6 & 30 & -215861 & 122. \\
\hline $1340-242$ & 89 & 7 & 31 & -186483 & 148 \\
\hline $1340-242$ & 89 & 8 & 31 & -160966 & 148 \\
\hline $1360-262$ & 89 & 9 & 30 & -146184 & 139 \\
\hline $1340-242$ & 89 & 10 & 31 & -179673 & 150 \\
\hline $1340-242$ & 89 & 11 & 30 & -119797 & 144 \\
\hline $1340-242$ & 89 & 12 & 30 & -85386 & 141 \\
\hline $22100-242$ & 89 & 1 & 28 & -52541 & 215 \\
\hline $2210-26,2$ & 89 & 2 & 28 & -28962 & 177 \\
\hline $22100-242$ & 89 & 3 & 28 & -93143 & 143 \\
\hline $2210-242$ & 89 & 4 & 30 & -15924 & 150 \\
\hline $2210-242$ & 89 & 5 & 31 & -13381 & 148 \\
\hline $2240-242$ & 89 & 6 & 30 & -13047 & 138 \\
\hline $2210-242$ & 89 & 7 & 31 & -11518 & 150 \\
\hline $2210-242$ & 89 & 8 & 31 & -12389 & 150 \\
\hline $22100-242$ & 89 & 9 & 30 & -12905 & 138 \\
\hline $2240-242$ & 89 & 10 & 31 & -11864 & 144 \\
\hline $22 W D-242$ & 89 & 11 & 30 & -13924 & 148 \\
\hline $2245-242$ & 89 & 12 & 31 & -11958 & 148 \\
\hline $2340-242$ & 89 & 1 & 31 & -126258 & 194 \\
\hline $23 W 0-24 Z$ & 89 & 2 & 28 & -110769 & 182 \\
\hline $2310-242$ & 89 & 3 & 29 & -59699 & 149 \\
\hline $23100-262$ & 89 & 4 & 30 & -35230 & 147 \\
\hline $2360-242$ & 89 & 5 & 31 & -28531 & $\{59$ \\
\hline $2340-242$ & 89 & 6 & 30 & -21348 & 167 \\
\hline
\end{tabular}


111. MAVAL PETROLEUM RESERVE NO. 1

WATER INJECTION SUMMARIES 1989

\begin{tabular}{|c|c|c|c|c|c|}
\hline WELL/SECT & YR & MONTH & $\begin{array}{l}\text { DAYS ON } \\
\text { INJECTION }\end{array}$ & $\begin{array}{l}\text { NATER } \\
\text { INJECTION }\end{array}$ & $\begin{array}{l}\text { TUBING } \\
\text { PRESSURE }\end{array}$ \\
\hline $2310-242$ & 89 & 7 & 31 & -13566 & 182 \\
\hline $2310-242$ & 89 & 8 & 13 & -6415 & 186 \\
\hline 23100-242 & 89 & 9 & 7 & -1683 & 153 \\
\hline $2310-242$ & 89 & 10 & 0 & 0 & 160 \\
\hline $2310-242$ & 89 & 11 & 0 & 0 & 153 \\
\hline $23100-242$ & 89 & 12 & 17 & -8704 & 153 \\
\hline $2660-242$ & 89 & 1 & 17 & -122251 & 181 \\
\hline $2410-242$ & 89 & 2 & 28 & -258510 & 144 \\
\hline $2440-242$ & 89 & 3 & 31 & -318085 & 137 \\
\hline $244 D-242$ & 89 & 4 & 27 & -297070 & 142 \\
\hline $2440-242 Z$ & 89 & 5 & 31 & -412383 & 117 \\
\hline $24100-242$ & 89 & 6 & 30 & -388866 & 113 \\
\hline $2440-242 Z$ & 89 & 7 & 31 & -551413 & 114 \\
\hline $2440-262$ & 89 & 8 & 31 & -516071 & 918 \\
\hline $2440-242$ & 89 & 9 & 25 & -272320 & 115 \\
\hline $24 \mathrm{KD}-242$ & 89 & 10 & 10 & -87527 & 126 \\
\hline $2440-242$ & 89 & 11 & 0 & 0 & 153 \\
\hline $2440-242$ & 89 & 12 & 0 & 0 & 0 \\
\hline $22400-242$ & 89 & 1 & 0 & 0 & 0 \\
\hline $22410-242$ & 89 & 2 & 0 & 0 & 0 \\
\hline $22410-242$ & 89 & 3 & 0 & 0 & 0 \\
\hline $22440-242$ & 89 & 4 & 0 & 0 & 0 \\
\hline $224100-21,2$ & 89 & 5 & 0 & 0 & 0 \\
\hline $22410-242$ & 89 & 6 & 0 & 0 & 0 \\
\hline $22440-242$ & 89 & 7 & 0 & 0 & 0 \\
\hline $224100-242$ & 89 & 8 & 0 & 0 & 0 \\
\hline $22460-242$ & 89 & 9 & 0 & 0 & 0 \\
\hline $224100-242$ & 89 & 10 & 0 & 0 & 0 \\
\hline $22440-242$ & 89 & 1 & 30 & -217470 & 128 \\
\hline $22440-242$ & 89 & 12 & 31 & -321388 & 77 \\
\hline $5140-262$ & 89 & 1 & 30 & -90869 & 107 \\
\hline $5160-262$ & 89 & 2 & 28 & -97052 & 107 \\
\hline $5140-262$ & 89 & 3 & 31 & -118040 & 81 \\
\hline $5110-262$ & 89 & 4 & 30 & -94320 & 101 \\
\hline $51 w 0-262$ & 89 & 5 & 28 & -121474 & 107 \\
\hline $5110-262$ & 89 & 6 & 30 & -120228 & 29 \\
\hline $5140-262$ & 89 & 7 & 31 & -115543 & 84 \\
\hline $5140-262$ & 89 & 8 & 30 & -61805 & 62 \\
\hline $5160-262$ & 89 & 9 & 30 & -64552 & 62 \\
\hline $5140-262$ & 89 & 10 & 28 & -72119 & 112 \\
\hline $5140-262$ & 89 & 11 & 29 & -79190 & 64 \\
\hline $5160-262$ & 89 & 12 & 29 & -77139 & 127 \\
\hline $3-7240-262$ & 89 & 9 & 0 & 0 & 0 \\
\hline $3-7240-262$ & 89 & 2 & 0 & 0 & 0 \\
\hline $3-7210-262$ & 89 & 3 & 0 & 0 & 0 \\
\hline 3-7ZW-262 & 89 & 4 & 0 & 0 & 0 \\
\hline
\end{tabular}


III. NAVAL PETROLELM RESERVE HO. 1

WATER INJECTIOW SUMMARIES 1989

\begin{tabular}{|c|c|c|c|c|c|}
\hline WELL/SECT & YR & MONTH & $\begin{array}{l}\text { DAYS ON } \\
\text { INJECT ION }\end{array}$ & $\begin{array}{l}\text { WATER } \\
\text { INJECTYON }\end{array}$ & $\begin{array}{l}\text { TUBING } \\
\text { PRESSURE }\end{array}$ \\
\hline $3-7210-262$ & 89 & 5 & 0 & 0 & 0 \\
\hline $3-7210-26 z$ & 89 & 6 & 0 & 0 & 0 \\
\hline $3-\mathrm{rZwD} \cdot 262$ & 89 & 7 & 0 & 0 & 0 \\
\hline $3-7210-262$ & 89 & 8 & 20 & -42125 & 38 \\
\hline $3-72100-262$ & 89 & 9 & 30 & -28400 & 115 \\
\hline $3-7210-262$ & 89 & 10 & 31 & -10393 & 115 \\
\hline $3-7210-26 z$ & 89 & 11 & 29 & -7829 & 63 \\
\hline $3-7240-26 z$ & 89 & 12 & 20 & -6399 & 128 \\
\hline $313 L-26$ & 89 & 1 & 31 & -17927 & 2987 \\
\hline $313 L-2 G$ & 89 & 2 & 28 & -14293 & 2995 \\
\hline $313 L-2 G$ & 89 & 3 & 31 & -14917 & 3010 \\
\hline $313 L-2 G$ & 89 & 4 & 30 & -13758 & 3093 \\
\hline $313 L-2 G$ & 89 & 5 & 31 & -13284 & 3098 \\
\hline $313 L-2 G$ & 89 & 6 & 30 & -11547 & 3095 \\
\hline $313 L-2 G$ & 89 & 7 & 31 & -9455 & 3058 \\
\hline $313 L-26$ & 89 & 8 & 31 & -9304 & 3100 \\
\hline $313 L-26$ & 89 & 9 & 26 & -7982 & 3098 \\
\hline $313 L-2 G$ & 89 & 10 & 25 & -6501 & 3000 \\
\hline $313 L-2 G$ & 89 & 11 & 19 & -6818 & 3021 \\
\hline $313 L-2 G$ & 89 & 12 & 30 & -9673 & 3005 \\
\hline $3130-2 G$ & 89 & 1 & 28 & -104878 & 2823 \\
\hline $313 U-26$ & 89 & 2 & 28 & -97587 & 2784 \\
\hline $3130-26$ & 89 & 3 & 31 & -112234 & 2931 \\
\hline $3130-26$ & 89 & 4 & 30 & -107146 & 2960 \\
\hline $313 U-26$ & 89 & 5 & 31 & -100903 & 2952 \\
\hline $3130-26$ & 89 & 6 & 30 & -104273 & 2860 \\
\hline $313 U-26$ & 89 & $y$ & 31 & -100187 & 2952 \\
\hline $3130-26$ & 89 & 8 & 31 & -107494 & 3018 \\
\hline $313 U-26$ & 89 & 9 & 26 & -74884 & 2573 \\
\hline $313 U-2 G$ & 89 & 10 & 25 & -70896 & 2571 \\
\hline $313 U-26$ & 89 & 11 & 19 & -70588 & 2800 \\
\hline $313 u-26$ & 89 & 12 & 30 & -104785 & 2850 \\
\hline $324-26$ & 89 & 1 & 31 & -109646 & 2873 \\
\hline $324-20$ & 89 & 2 & 28 & -94389 & 2859 \\
\hline $324-26$ & 89 & 3 & 31 & -105820 & 2956 \\
\hline $324-26$ & 89 & 4 & 30 & -102238 & 3023 \\
\hline $324-26$ & 89 & 5 & 31 & -100757 & 3032 \\
\hline $3214-26$ & 89 & 6 & 30 & -88508 & 2993 \\
\hline $324-26$ & 89 & 7 & 31 & -90471 & 3002 \\
\hline $324-26$ & 89 & 8 & 31 & -88274 & 2973 \\
\hline $324-2 G$ & 89 & 9 & 25 & -52984 & 2602 \\
\hline $324-2 G$ & 89 & 10 & 25 & -73396 & 2888 \\
\hline $324-20$ & 89 & 11 & 19 & -591489 & 2845 \\
\hline $324-20$ & 89 & 12 & 30 & -90739 & 2947 \\
\hline $333 L-2 G$ & 89 & 1 & 31 & -22283 & 3024 \\
\hline $333 L-2 G$ & 88 & 2 & 28 & -14755 & 2939 \\
\hline
\end{tabular}


111. NAVAL PETROLEUM RESERVE NO. 1 WATER INJECTION SUMMARIES 1989

\begin{tabular}{|c|c|c|c|c|c|}
\hline WELL/SECT & YR & MONTH & $\begin{array}{l}\text { DAYS ON } \\
\text { INJECTION }\end{array}$ & $\begin{array}{l}\text { WATER } \\
\text { INJECTION }\end{array}$ & $\begin{array}{l}\text { TUBING } \\
\text { PRESSURE }\end{array}$ \\
\hline $333 L-2 G$ & 89 & 3 & 31 & -17962 & 2985 \\
\hline $333 L-26$ & 89 & 4 & 30 & -18648 & 3000 \\
\hline $333 L-2 G$ & 89 & 5 & 31 & -18411 & 2990 \\
\hline $333 L-2 G$ & 89 & 6 & 30 & -18185 & 2982 \\
\hline $333 L-2 G$ & 89 & 7 & 31 & -18317 & 3015 \\
\hline $333 L-2 G$ & 89 & 8 & 31 & -19077 & 2998 \\
\hline $333 L-2 G$ & 89 & 9 & 26 & -17353 & 2798 \\
\hline $333 L-2 G$ & 89 & 10 & 31 & -23329 & 2573 \\
\hline $333 L-20$ & 89 & 11 & 23 & -20825 & 2533 \\
\hline $333 L-26$ & 89 & 12 & 30 & -37715 & 2885 \\
\hline $333 U-26$ & 89 & 1 & 31 & -109816 & 2852 \\
\hline $333 U-26$ & 89 & 2 & 28 & -96153 & 2802 \\
\hline $3330-2.6$ & 89 & 3 & 31 & -107339 & 2927 \\
\hline $333 U-20$ & 89 & 4 & 30 & -101493 & 3000 \\
\hline $333 u-26$ & 89 & 5 & 31 & -102695 & 2889 \\
\hline $333 U-26$ & 89 & 6 & 30 & -95775 & 2827 \\
\hline $333 u-26$ & 89 & 7 & 31 & -94004 & 2877 \\
\hline $333 v-20$ & 89 & 8 & 31 & -97056 & 2879 \\
\hline $333 U-20$ & 89 & 9 & 26 & -62986 & 2556 \\
\hline $333 u-20$ & 89 & 10 & 26 & -74289 & 2675 \\
\hline $333 U-26$ & 89 & 11 & 24 & -61546 & 2543 \\
\hline $333 u-20$ & 89 & 12 & 30 & -73076 & 2760 \\
\hline $352-26$ & 89 & 1 & 31 & -26609 & 3019 \\
\hline $352-26$ & 89 & 2 & 28 & -23813 & 3063 \\
\hline $352-2 G$ & 89 & 3 & 31 & -21984 & 2995 \\
\hline $352-2 G$ & 89 & 4 & 30 & -21190 & 3000 \\
\hline $352-20$ & 89 & 5 & 31 & -19967 & 3003 \\
\hline $352-26$ & 89 & 6 & 30 & $-187 / 63$ & 3000 \\
\hline $352-2.6$ & 89 & 7 & 31 & .97980 & 3019 \\
\hline $352-20$ & 89 & 8 & 31 & -18518 & 3005 \\
\hline $352-2 G$ & 89 & 9 & 26 & -15727 & 3000 \\
\hline $352-2 G$ & 89 & 10 & 25 & -12113 & 2935 \\
\hline $352-2 G$ & 89 & 11 & 19 & -11288 & 2880 \\
\hline $352-20$ & 89 & 12 & 30 & -17721 & 2918 \\
\hline $353-2 G$ & 89 & 1 & 31 & -118973 & 2895 \\
\hline $353-26$ & 89 & 2 & 28 & -903653 & 2868 \\
\hline $353-2 G$ & 89 & 3 & 31 & -116802 & 2924 \\
\hline $353-26$ & 89 & 4 & 30 & -107566 & 2988 \\
\hline $353-2 G$ & 89 & 5 & 31 & -104182 & 2971 \\
\hline $353-26$ & 89 & 6 & 30 & -103513 & 2950 \\
\hline $353-26$ & 89 & 7 & 31 & -79856 & 2765 \\
\hline $353-2 G$ & 89 & 8 & 31 & -100315 & 2937 \\
\hline $353-26$ & 89 & 9 & 27 & -72397 & 2746 \\
\hline $353-20$ & 89 & 10 & 25 & -71411 & 2721 \\
\hline $353-26$ & 89 & 11 & 19 & -69911 & 2863 \\
\hline $353-20$ & 89 & 12 & 30 & -104823 & 2930 \\
\hline
\end{tabular}


III. MAVAL PETROLEUM RESERVE wo. 1 WATER INUECTION SUMARRIES 1989

\begin{tabular}{|c|c|c|c|c|c|}
\hline EELL/SEET & YR & Mowrn & $\begin{array}{l}\text { DAYS ON } \\
\text { IWJECTION }\end{array}$ & $\begin{array}{l}\text { WUTER } \\
\text { INNECTION }\end{array}$ & $\begin{array}{l}\text { TUBSING } \\
\text { PRESSURE }\end{array}$ \\
\hline $371-26$ & 89 & 1 & 31 & .57366 & 3005 \\
\hline $379-20$ & 89 & 2 & 28 & -50422 & 7225 \\
\hline $371-26$ & 89 & $\dot{5}$ & 31 & -51556 & 3018 \\
\hline $371-26$ & 89 & 4 & 30 & $-4 r 243$ & 3012 \\
\hline $371-26$ & 89 & 5 & 31 & -47323 & 2992 \\
\hline $374-26$ & 89 & 6 & 30 & -164121 & 3010 \\
\hline $371-26$ & 89 & 7 & 31 & -38867 & 3002 \\
\hline $371-26$ & 8 & 8 & 31 & -61525 & 3005 \\
\hline $371-26$ & 89 & 0 & 2.6 & .55591 & 2987 \\
\hline $371-26$ & 89 & 10 & 28 & -30092 & 2900 \\
\hline $371-26$ & 89 & 11 & 9 & -33129 & 2987 \\
\hline $374-26$ & 89 & 12 & 30 & -46010 & 2077 \\
\hline $314-36$ & 89 & 1 & 31 & -19327 & 3003 \\
\hline $316-30$ & 89 & 2 & 28 & -16800 & 3000 \\
\hline $314 \cdot 36$ & 8 & 3 & 31 & - 17801 & 3000 \\
\hline $514-30$ & 89 & 4 & 30 & -10373 & 3010 \\
\hline $314-30$ & 80 & 5 & $3 i$ & -16295 & 3003 \\
\hline $314-30$ & 89 & 6 & 30 & -1524 & 3027 \\
\hline $316-36$ & 99 & 7 & 31 & -13150 & 3000 \\
\hline $314-36$ & $\infty 9$ & 8 & 31 & .13819 & 3013 \\
\hline $314-30$ & 89 & 9 & 26 & -11986 & 3013 \\
\hline $314-36$ & 89 & 10 & 25 & -12217 & 2050 \\
\hline $314 \cdot 36$ & 89 & 11 & 19 & -8134 & 2824 \\
\hline $314-36$ & 89 & 12 & 30 & .9795 & 2996 \\
\hline 315.36 & $\theta$ & 1 & 39 & .44710 & 3024 \\
\hline $315-36$ & 89 & 2 & 28 & -40230 & 3086 \\
\hline $345-36$ & 89 & 3 & 31 & .41637 & 3013 \\
\hline $395-30$ & $\theta$ & 4 & 30 & -39408 & 3000 \\
\hline $315-36$ & 89 & 5 & 31 & -35012 & 2992 \\
\hline $315-36$ & 89 & 6 & 30 & -39584 & 3023 \\
\hline $315-30$ & 9 & 7 & 31 & -38329 & 2997 \\
\hline $315-36$ & 89 & 8 & 31 & -40600 & 3032 \\
\hline $315-36$ & 89 & 9 & 26 & $.33 \pi T 7$ & 3004 \\
\hline $315-36$ & 89 & 10 & 25 & 30204 & 2879 \\
\hline $315 \cdot 36$ & 89 & 11 & 19 & -27253 & 2945 \\
\hline $315 \cdot 30$ & 89 & 12 & 29 & -41015 & 29997 \\
\hline $324-36$ & 89 & 1 & 31 & -51856 & 2991 \\
\hline $326-36$ & 80 & 2 & 28 & -47502 & 3011 \\
\hline $324-36$ & 89 & 3 & 31 & -52954 & 2976 \\
\hline $324-36$ & 89 & 4 & 30 & -51137 & 2990 \\
\hline $324-36$ & 80 & 5 & 31 & -50591 & 2998 \\
\hline $326-36$ & 20 & 6 & 30 & -52201 & 3012 \\
\hline $324 \cdot 36$ & 39 & 7 & 31 & .50611 & 3027 \\
\hline $324-36$ & 89 & 8 & 31 & -52205 & 3037 \\
\hline $326-36$ & 89 & 9 & 26 & -49600 & 2979 \\
\hline $324-36$ & 38 & 10 & 25 & -34342 & 2806 \\
\hline
\end{tabular}


11. MAVAL PETROLEUM RESEXVE MO. 1 MATER IMJECTION SUMARIES 1980

\begin{tabular}{|c|c|c|c|c|c|}
\hline WELL/SECT & YR & MONTH & $\begin{array}{l}\text { DAYS ON } \\
\text { INJECTION }\end{array}$ & $\begin{array}{c}\text { MATER } \\
\text { INJECTION }\end{array}$ & $\begin{array}{l}\text { TUBIHG } \\
\text { PRESSURE }\end{array}$ \\
\hline $324-3 G$ & 89 & 11 & 10 & -35358 & 2851 \\
\hline $324-36$ & 89 & 12 & 30 & .55630 & 2945 \\
\hline $355-30$ & 89 & 1 & 31 & -67376 & 2842 \\
\hline $335-36$ & 89 & 2 & 28 & -58102 & 3020 \\
\hline $535-36$ & 89 & 3 & 31 & -62886 & 3003 \\
\hline $335-36$ & 89 & 4 & 30 & -57476 & 2993 \\
\hline $335-36$ & 89 & 5 & 31 & -56358 & 2981 \\
\hline $335 \cdot 36$ & 88 & 6 & 30 & -54340 & 3000 \\
\hline $335-30$ & 89 & 7 & 31 & -59690 & 2992 \\
\hline $335-36$ & 89 & 8 & 31 & -54113 & 3011 \\
\hline $335-36$ & 89 & 9 & 26 & -44011 & 2894 \\
\hline $335-30$ & 89 & 10 & 25 & -39066 & 2898 \\
\hline $335-30$ & 89 & 11 & 19 & -37545 & 2947 \\
\hline $335-36$ & 89 & 12 & 30 & -54905 & 2952 \\
\hline $346-36$ & 89 & 1 & 31 & -86723 & 2863 \\
\hline $344-30$ & 89 & 2 & 28 & -76349 & 2989 \\
\hline 344 - 36 & 89 & 3 & 31 & -83200 & 2984 \\
\hline $364-3 x=$ & 89 & 4 & 29 & $-\pi 796$ & 2092 \\
\hline $364-30$ & 89 & 5 & 39 & .82794 & 2987 \\
\hline $344-36$ & 89 & 6 & 30 & -80914 & 3000 \\
\hline $346-36$ & 89 & 7 & 34 & .76740 & 2997 \\
\hline $344-36$ & 69 & 8 & 24 & $-60 \mathrm{rr}$ & 3024 \\
\hline $344 \cdot 30$ & $B 9$ & 9 & 22 & -52277 & 2540 \\
\hline $344-36$ & 69 & 10 & 25 & -63368 & 2860 \\
\hline 344.36 & 89 & 11 & 19 & .51188 & 2761 \\
\hline $344-36$ & $B P$ & 12 & 30 & -76637 & 2860 \\
\hline $354-36$ & 89 & 1 & 31 & " 16804 & 3097 \\
\hline $354-36$ & 89 & 2 & 28 & -14721 & 3086 \\
\hline $354-36$ & 89 & 3 & 31 & -96168 & 2950 \\
\hline $354-36$ & 89 & 4 & 30 & -81731 & 2982 \\
\hline $354-36$ & 89 & 5 & 31 & -11209 & 2966 \\
\hline $354-36$ & 89 & 6 & 30 & -11698 & 2997 \\
\hline $354-36$ & 89 & 7 & 39 & -12214 & 3032 \\
\hline $354-36$ & 80 & 8 & 24.4 & -10273 & 3048 \\
\hline $354-30$ & 89 & 9 & 21 & -10847 & 22609 \\
\hline $354-36$ & 89 & 10 & 25 & -9087 & 2810 \\
\hline $354-30$ & 89 & 11 & 30 & -8645 & 2734 \\
\hline $354-36$ & 89 & 12 & 30 & -14460 & 2008 \\
\hline $364-36$ & 89 & 9 & 31 & -94071 & 2946 \\
\hline $364-30$ & 89 & 2 & 28 & -80116 & $2 \$ 71$ \\
\hline $364-36$ & 09 & 3 & 39 & -82967 & 2979 \\
\hline $364-30$ & 89 & 4 & 30 & -23207 & 2975 \\
\hline $364 \cdot 30$ & 89 & 5 & 31 & -83017 & 2867 \\
\hline $364-30$ & 88 & 6 & 30 & -78038 & 29,55 \\
\hline $364-30$ & 89 & 7 & 31 & -77320 & 2095 \\
\hline $364-30$ & 89 & 8 & 17 & -42349 & 2984 \\
\hline
\end{tabular}


III. MAVAL PETROLeUn Reserve No. 1 WRTER INJECTION SUMARIES 1989

\begin{tabular}{|c|c|c|c|c|c|}
\hline YELL/SECT & YR & MONYH & $\begin{array}{l}\text { DAYS ON } \\
\text { IWJECTION }\end{array}$ & $\begin{array}{l}\text { WATER } \\
\text { IHJECTIOH }\end{array}$ & $\begin{array}{l}\text { TUBING } \\
\text { PRESSURE }\end{array}$ \\
\hline ….........non & $\cdots$ & $\cdots$ & $\cdots$ & $\ldots \ldots$ & ……........ \\
\hline $364-36$ & 89 & 9 & 21 & -55765 & 2593 \\
\hline $364-30$ & 89 & 10 & 25 & -66672 & 2635 \\
\hline $3 t: h-36$ & 89 & 19 & 30 & -56683 & 2655 \\
\hline $364-36$ & 89 & 12 & 30 & -87709 & 2558 \\
\hline $384 L-36$ & 89 & 1 & 31 & -14667 & 2005 \\
\hline $384 L-30$ & 89 & 2 & 28 & -12549 & 2902 \\
\hline $384 L-36$ & 89 & 3 & 31 & -13952 & 3000 \\
\hline $384 L-36$ & 89 & 4 & 30 & -12379 & 3000 \\
\hline $384 L-3 G$ & 89 & 5 & 31 & -12567 & 3000 \\
\hline $304 L-30$ & 89 & 6 & 30 & -12192 & 3000 \\
\hline $384 L-36$ & 89 & 7 & 31 & -11158 & 3018 \\
\hline $384 L-36$ & 89 & 8 & 24 & -8858 & 3000 \\
\hline $3846-36$ & 89 & 9 & 0 & 0 & 3000 \\
\hline $384 L-30$ & 89 & 10 & 26 & -17076 & 2746 \\
\hline $384 L-30$ & 89 & 11 & 27 & -30442 & 3027 \\
\hline $3846-36$ & 89 & 92 & 25 & -26216 & 2948 \\
\hline $3040-36$ & 89 & 1 & 31 & -93580 & 2923 \\
\hline $38401-36$ & 89 & 2 & 28 & -82814 & 2914 \\
\hline $3840-36$ & 89 & 3 & 31 & -106896 & 2855 \\
\hline $3840 \mathrm{U}-36$ & 89 & 4 & 30 & -902191 & 2817 \\
\hline $3840-36$ & 89 & 5 & 31 & -105180 & 2889 \\
\hline $3864-36$ & 88 & 6 & 30 & -95215 & 2885 \\
\hline $3840-3 a$ & 89 & 7 & 31 & -99272 & 2900 \\
\hline $384 \mathrm{U} \cdot 30$ & 89 & 8 & 24 & -85994 & 2857 \\
\hline $384 U-50$ & 89 & 9 & 0 & 0 & 2857 \\
\hline $3940-36$ & 89 & 10 & 26 & -83240 & 2667 \\
\hline $3844-36$ & 89 & 11 & 30 & -135764 & $285 \%$ \\
\hline $3814 \cdot 36$ & 89 & 12 & 25 & $-104 \mathrm{Tr}$ & 2833 \\
\hline $385-46$ & 89 & 1 & 31 & -41965 & 3019 \\
\hline $315-40$ & 89 & 2 & 28 & -32742 & 3000 \\
\hline $315-46$ & 89 & 3 & 31 & -33508 & 3013 \\
\hline $395-46$ & 89 & 4 & 30 & -29441 & 3005 \\
\hline $315-h, 6$ & 89 & 5 & 31 & -29396 & 3003 \\
\hline $315-46$ & 89 & 6 & 30 & -28842 & 3000 \\
\hline $345-46$ & 89 & 7 & 31 & -27123 & 3002 \\
\hline $315-40$ & 89 & 8 & 30 & -25663 & 3026 \\
\hline $315-46$ & 89 & 9 & 26 & -24899 & 3013 \\
\hline $315-40$ & 8 & 10 & 31 & -23115 & 2911 \\
\hline $315-46$ & 80 & 19 & 30 & -25108 & 2970 \\
\hline $315-46$ & 80 & 12 & 30 & -27274 & 2988 \\
\hline $326-46$ & 89 & 1 & 39 & -50458 & $\$ 037$ \\
\hline $326 \cdot 46$ & 89 & 2 & 28 & -50413 & 3063 \\
\hline $326-60$ & 89 & 3 & 31 & -53287 & 3013 \\
\hline $326-46$ & 89 & 4 & 30 & -50390 & 3000 \\
\hline $326-46$ & 0 & 5 & 31 & -47885 & 2969 \\
\hline $326-40$ & 80 & 6 & 30 & -46692 & 2998 \\
\hline
\end{tabular}


111. haval petroleun Reserve nO. 1 WATER INJECTION SUMURIES 9989

\begin{tabular}{|c|c|c|c|c|c|}
\hline WELL.SECT & YR & MONTH & $\begin{array}{l}\text { DAYS ON } \\
\text { INJECTIOA }\end{array}$ & $\begin{array}{c}\text { WATER } \\
\text { INJECTION }\end{array}$ & $\begin{array}{l}\text { TUBING } \\
\text { PRESSURE }\end{array}$ \\
\hline $326-40$ & 89 & 7 & 31 & .47256 & 3000 \\
\hline $326-46$ & 89 & 8 & 30 & -47038 & 3000 \\
\hline $326-46$ & 89 & D & 26 & -40707 & 2988 \\
\hline $326-40$ & 89 & 10 & 31 & -43746 & 2915 \\
\hline $326-46$ & 89 & 11 & 30 & -45661 & 2990 \\
\hline $326-46$ & 89 & 12 & 30 & -42798 & 2997 \\
\hline $335-46$ & 89 & 1 & 31 & -87642 & 2916 \\
\hline $335-40$ & 89 & 2 & 28 & $-7,260$ & 2923 \\
\hline $335-46$ & 89 & 3 & 31 & -8.2553 & 2982 \\
\hline $335-46$ & 89 & 4 & 30 & -77594 & 3093 \\
\hline $335-46$ & 89 & 5 & 31 & -77081 & 2977 \\
\hline $335-46$ & 89 & 6 & 30 & -75129 & 3000 \\
\hline $335-46$ & 89 & 7 & 31 & -70754 & 2997 \\
\hline $335-46$ & 89 & 8 & 31 & -67103 & 3000 \\
\hline $335-46$ & 89 & 9 & 26 & -62027 & 2977 \\
\hline $335-46$ & 89 & 10 & 31 & .62972 & 2890 \\
\hline $335-46$ & 89 & 11 & 30 & -7277 & 2948 \\
\hline $335-40$ & 89 & 12 & 30 & -69125 & 2947 \\
\hline $346 L-4 G$ & 89 & 1 & 31 & -13785 & 3102 \\
\hline $346 L-4 G$ & 89 & 2 & 28 & -12179 & 3105 \\
\hline $346 L-40$ & 89 & 3 & 31 & $-9<46$ & 2948 \\
\hline $346 L-46$ & 89 & 4 & 30 & -9766 & 2992 \\
\hline $346 L-46$ & 89 & 5 & 31 & -10535 & 2823 \\
\hline $346 L-46$ & 89 & 6 & 30 & -9926 & 2983 \\
\hline $366 L-40$ & 89 & 7 & 31 & .9610 & 3000 \\
\hline $346 L-4 G$ & 80 & 8 & 31 & -9367 & 3016 \\
\hline $346 L-4 G$ & 89 & 9 & 26 & -7045 & 2987 \\
\hline $346 L-46$ & 89 & 10 & 31 & -8125 & 2903 \\
\hline $346 L-4 G$ & 89 & 11 & 30 & -8694 & 3003 \\
\hline $346 L-46$ & 89 & 12 & 30 & -8658 & 2997 \\
\hline $34600-40$ & 89 & 1 & 31 & -33522 & 3102 \\
\hline $3460-4 a$ & 89 & 2 & 28 & -26404 & 3102 \\
\hline $346 \mathrm{U}-46$ & 80 & 3 & 31 & -23594 & 2939 \\
\hline $34520-46$ & 80 & 4 & 30 & -22590 & 3007 \\
\hline $3.66 U-4 G$ & 89 & 5 & 20 & -14823 & 3030 \\
\hline $346 U-46$ & 89 & 6 & 30 & -19259 & 3000 \\
\hline $3461-46$ & 89 & 7 & 31 & -98103 & 3003 \\
\hline $34601-46$ & 89 & 8 & 31 & -16870 & 3016 \\
\hline $34601-1.6$ & 88 & 9 & 26 & -12693 & 2994 \\
\hline $346 U-4 G$ & 89 & 10 & 31 & -13163 & 2913 \\
\hline $34613-40$ & 89 & 11 & 30 & -12.628 & 3008 \\
\hline $3460-460$ & 89 & 12 & 30 & .19119 & 2998 \\
\hline $355-40$ & 89 & 1 & 31 & -59276 & 3000 \\
\hline $355-40$ & 89 & 2 & 28 & -48294 & 3025 \\
\hline $355-40$ & 89 & 3 & 31 & -48636 & 3021 \\
\hline $355-46$ & 89 & 4 & 30 & -36308 & 2993 \\
\hline
\end{tabular}


111. MAVAL PETROLEUN REserve No. 1 WATER INJECTION SUMARIES 190,

\begin{tabular}{|c|c|c|c|c|c|}
\hline WELL.SEECT & $Y R$ & MONTH & $\begin{array}{l}\text { DAYS ON } \\
\text { INJECTION }\end{array}$ & $\begin{array}{c}\text { WATER } \\
\text { INJECTIOH }\end{array}$ & $\begin{array}{l}\text { TUBING } \\
\text { PRESSURE }\end{array}$ \\
\hline $355-46$ & 89 & 5 & 12 & -15718 & 2900 \\
\hline $355-40$ & 89 & 6 & 30 & -34297 & 3018 \\
\hline $355-40$ & 89 & 7 & 31 & -30531 & 3076 \\
\hline $355-40$ & 89 & 8 & 31 & -32021 & 3090 \\
\hline $355-46$ & 89 & 9 & 26 & -25963 & 3087 \\
\hline $355-46$ & 89 & 10 & 31 & -27960 & 3000 \\
\hline $355-46$ & 89 & 19 & 30 & -30727 & 3081 \\
\hline $355-46$ & 89 & 12 & 30 & -31107 & 3088 \\
\hline $364-46$ & 89 & 1 & 39 & -99098 & 2945 \\
\hline $364-40$ & 89 & 2 & 2.8 & -83949 & 2975 \\
\hline $364-46$ & 89 & 3 & 31 & -94655 & 2987 \\
\hline $364-66$ & 89 & 4 & 30 & -91949 & 2987 \\
\hline $364-40$ & 89 & 5 & 31 & -92681 & 3013 \\
\hline $364-46$ & 89 & 6 & 30 & -86575 & 3000 \\
\hline $364-46$ & 89 & $T$ & 31 & -84877 & 3000 \\
\hline $364-46$ & 89 & 8 & 31 & -80426 & 2800 \\
\hline $364-46$ & 89 & 9 & 25 & -56605 & 2629 \\
\hline $364-46$ & 89 & 10 & 31 & -82173 & 2911 \\
\hline $364-40$ & 89 & 11 & 30 & -78435 & 2960 \\
\hline $364-46$ & 89 & 12 & 30 & -75224 & 2952 \\
\hline $365 L-40$ & 89 & 1 & 0 & 0 & 2977 \\
\hline $365 L-40$ & 89 & 2 & 0 & 0 & $29 \pi$ \\
\hline $365 L-40$ & 89 & 3 & 8 & -5017 & 2988 \\
\hline $365 L-46$ & 89 & 4 & 30 & -15043 & 2993 \\
\hline $365 L-40$ & 89 & 5 & 29 & -14597 & 3020 \\
\hline $365 L-46$ & 89 & 6 & 25 & -4570 & 3025 \\
\hline $365 L-4 G$ & 89 & 7 & 0 & 0 & 3025 \\
\hline $365 L-4 G$ & 89 & 8 & 6 & -1627 & 3027 \\
\hline $365 L-46$ & 89 & 9 & 26 & -8043 & 3058 \\
\hline $365 L-4 G$ & 89 & 10 & 31 & -8569 & 2955 \\
\hline $365 L-46$ & 89 & 19 & 23 & .6127 & 2910 \\
\hline $365 L-40$ & 89 & 12 & 30 & -6939 & 2998 \\
\hline $365 U-46$ & 89 & 1 & 31 & -17541 & 3048 \\
\hline $365 U-46$ & 89 & 2 & 28 & -14390 & 3095 \\
\hline $365 U-40$ & 69 & 3 & 29 & -13436 & 3010 \\
\hline $365 U-46$ & 89 & 4 & 30 & -11842 & 3003 \\
\hline $365 U-40$ & 89 & 5 & 18 & -7781 & 2976 \\
\hline $365 U-46$ & 89 & 6 & 30 & -18899 & 2953 \\
\hline $365 \omega-46$ & 89 & 7 & 31 & -18333 & 2963 \\
\hline $365 U-46$ & 89 & 8 & 31 & -17008 & 2960 \\
\hline $365 U-46$ & 89 & 9 & 26 & -10697 & 3002 \\
\hline $365 U-40$ & 88 & 10 & 31 & -11630 & 2916 \\
\hline $365 U-4 G$ & 89 & 11 & 25 & -10539 & 28302 \\
\hline $3650-46$ & 69 & 12 & 30 & -11269 & 2887 \\
\hline $375-4, C$ & 89 & 1 & 34 & -30473 & 3074 \\
\hline $375-46$ & 89 & 2 & 28 & -26959 & 3114 \\
\hline
\end{tabular}


III. MAVAL PETROLEUM RESERVE MO. 1 WATER INJECTION SUMMARIES 1989

\begin{tabular}{|c|c|c|c|c|c|}
\hline MELL/SECT & YR & MONTH & $\begin{array}{l}\text { DAYS ON } \\
\text { INJECTION }\end{array}$ & $\begin{array}{c}\text { WATER } \\
\text { INJECTION }\end{array}$ & $\begin{array}{l}\text { TUBING } \\
\text { PRESSURE }\end{array}$ \\
\hline $375-46$ & 89 & 3 & 31 & -24494 & 3008 \\
\hline $375-46$ & 89 & 4 & 30 & -21723 & 3005 \\
\hline $375-46$ & 89 & 5 & 31 & .21546 & 3003 \\
\hline $375-46$ & 89 & 6 & 30 & -20908 & 3005 \\
\hline $373-46$ & 89 & 7 & 39 & -20782 & 3000 \\
\hline $375-46$ & 89 & 8 & 31 & -20808 & 3011 \\
\hline $375-46$ & 89 & 9 & 26 & -18424 & 2988 \\
\hline $375-46$ & 89 & 10 & 31 & -21265 & 2932 \\
\hline $375-46$ & 89 & 11 & 30 & -21118 & 2952 \\
\hline $375-46$ & 89 & 12 & 30 & -20693 & 2955 \\
\hline $384-40$ & 89 & 1 & 31 & -61838 & 3092 \\
\hline $384-46$ & 89 & 2 & 28 & -51556 & 3098 \\
\hline $384-46$ & 89 & 3 & 31 & -51010 & 3037 \\
\hline $384-46$ & 89 & 4 & 30 & -49372 & 3075 \\
\hline $384-4 G$ & 89 & 5 & 31 & -48176 & 3094 \\
\hline $384-4 G$ & 89 & 6 & 30 & -50006 & 3100 \\
\hline $384-46$ & 84 & 7 & 31 & -46016 & 3094 \\
\hline $384-46$ & 89 & 8 & 31 & -45972 & 3100 \\
\hline $384-40$ & 89 & 9 & 26 & -38283 & 3083 \\
\hline $384-4 G$ & 89 & 10 & 31 & -45662 & 2997 \\
\hline $384-46$ & 89 & 11 & 30 & -52381 & 3037 \\
\hline $384-60$ & 89 & 12 & 30 & -47899 & 3020 \\
\hline $313-56$ & 89 & 1 & 23 & -67396 & 2961 \\
\hline $313-56$ & 89 & 2 & 28 & -97792 & 2809 \\
\hline $313-56$ & 89 & 3 & 31 & -113589 & 2941 \\
\hline $313-56$ & 89 & 4 & 30 & -102619 & 2945 \\
\hline $343-56$ & 89 & 5 & 31 & -102018 & 2950 \\
\hline $313-56$ & 89 & 6 & 30 & -99596 & 2945 \\
\hline $313-56$ & 89 & 7 & 31 & -89944 & 2919 \\
\hline $313 \cdot 56$ & 89 & $B$ & 31 & .95631 & 2969 \\
\hline $313-56$ & 89 & 9 & 26 & -64617 & 2708 \\
\hline $313-56$ & 89 & 10 & 31 & -87236 & 2874 \\
\hline $313-5 a$ & 89 & 11 & 30 & -82748 & 2913 \\
\hline 313.56 & 89 & 12 & 30 & -80854 & 2938 \\
\hline $314 x-50$ & 89 & 1 & 31 & -57279 & 3003 \\
\hline $314 x-50$ & 89 & 2 & 28 & -45207 & 3016 \\
\hline $314 x-50$ & 89 & 3 & 31 & -48473 & 3000 \\
\hline $314 x-50$ & 89 & 4 & 30 & -44095 & 3000 \\
\hline $314 x-50$ & 89 & 5 & 31 & -44577 & 3003 \\
\hline $314 x-56$ & 89 & 6 & 30 & -42750 & 3002 \\
\hline $314 x-5 G$ & 89 & 7 & 31 & -42291 & 2997 \\
\hline $314 x-50$ & 89 & 8 & 31 & -42020 & 3011 \\
\hline $314 x-56$ & 89 & 9 & 26 & -37327 & 29144 \\
\hline $314 x-50$ & 89 & 10 & 31 & -39329 & 2876 \\
\hline $314 x-56$ & 89 & 11 & 30 & -42398 & 2960 \\
\hline $314 x-50$ & 89 & 12 & 30 & -42340 & 2958 \\
\hline
\end{tabular}


111. MAVAL PETROLEUM RESERVE HO. 1 WATER INJECTION SUMARIES 1989

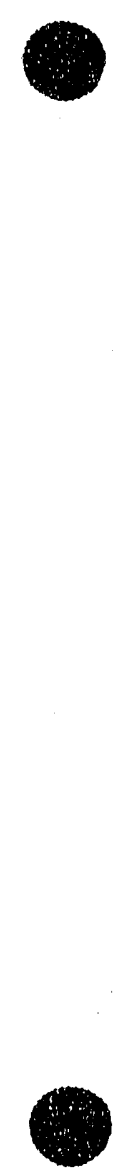

\begin{tabular}{|c|c|c|c|c|c|}
\hline WELL/SECT & YR & MONTH & $\begin{array}{l}\text { DAYS CH } \\
\text { IMJECTION }\end{array}$ & $\begin{array}{c}\text { WATER } \\
\text { INJECTION }\end{array}$ & $\begin{array}{l}\text { TUBING } \\
\text { PRESSURE }\end{array}$ \\
\hline $324 x-56$ & 89 & 9 & 31 & -65400 & 3008 \\
\hline $324 x-56$ & 89 & 2 & 28 & -51247 & 2991 \\
\hline $324 x-56$ & 89 & 3 & 31 & -54111 & 3005 \\
\hline $324 x-56$ & 89 & 4 & 30 & -48019 & 3008 \\
\hline $324 x-56$ & 89 & 5 & 31 & -49105 & 3000 \\
\hline $324 x-56$ & 89 & 6 & 30 & -46068 & 3000 \\
\hline $324 x=56$ & 89 & 7 & 31 & -44696 & 2997 \\
\hline $324 x-56$ & 89 & 8 & 31 & -45250 & 3000 \\
\hline $324 x-56$ & 89 & 9 & 26 & -39774 & 2988 \\
\hline $324 x-56$ & 89 & 10 & 31 & -44181 & 2905 \\
\hline $324 x-56$ & 89 & 11 & 30 & -45604 & 2988 \\
\hline $324 x-50$ & 89 & 12 & 30 & -45870 & 2988 \\
\hline $335 A-5 G$ & 89 & 1 & 31 & -20095 & 3019 \\
\hline $335 A-56$ & 89 & 2 & 28 & -15672 & 3000 \\
\hline $335 A-56$ & 89 & 3 & 39 & -16394 & 3008 \\
\hline $335 A-56$ & 89 & 4 & 30 & -15186 & 3005 \\
\hline $335 A-5 G$ & 89 & 5 & 31 & -15430 & 3002 \\
\hline $335 A-56$ & 89 & 6 & 30 & -14629 & 3000 \\
\hline $335 A-5 G$ & 89 & 7 & 31 & -14069 & 3000 \\
\hline $335 A-56$ & 89 & 8 & 20 & -9998 & 3000 \\
\hline $335 A-56$ & 89 & 9 & 26 & -14792 & 3027 \\
\hline $335 A-50$ & 89 & 10 & 31 & -14422 & 2920 \\
\hline $335 A-50$ & 89 & 11 & 29 & $-14 \% 68$ & 2997 \\
\hline $335 A-5 G$ & 89 & 12 & 27 & -12371 & 2954 \\
\hline $344-5 G$ & 89 & 1 & 31 & -59905 & 2961 \\
\hline $344-56$ & 89 & 2 & 28 & -47586 & 2980 \\
\hline $344-56$ & 89 & 3 & 31 & -50324 & 2977 \\
\hline $344-50$ & 89 & 4 & 30 & -1.6093 & 2857 \\
\hline $344-56$ & 89 & 5 & 31 & -51596 & 3006 \\
\hline $344-5 C$ & 89 & 6 & 30 & -48278 & 3002 \\
\hline $344-56$ & 89 & 7 & 31 & -45349 & 2998 \\
\hline $344-56$ & 89 & 8 & 31 & -47094 & 3008 \\
\hline $344-56$ & 89 & 9 & 26 & -37101 & 2840 \\
\hline $344-5 G$ & 89 & 10 & 31 & -42673 & 2896 \\
\hline $364-56$ & 89 & 11 & 30 & -45194 & 2962 \\
\hline $344-50$ & 89 & 12 & 30 & -45707 & 2988 \\
\hline $355 x-56$ & 89 & 1 & 31 & -42589 & 3006 \\
\hline $355 x-56$ & 89 & 2 & 28 & -36655 & 3000 \\
\hline $355 x-56$ & 89 & 3 & 31 & -40661 & 3006 \\
\hline $355 x-56$ & 89 & 4 & 30 & -37050 & 3010 \\
\hline $355 x-56$ & 89 & 5 & 31 & -35239 & 3010 \\
\hline $355 x-56$ & 89 & 6 & 30 & -33599 & 3000 \\
\hline $355 x-56$ & 89 & 7 & 31 & -33184 & 2981 \\
\hline $355 x-50$ & 89 & 8 & 31 & $-\$ 4 B 10$ & 3000 \\
\hline $355 x-56$ & 89 & 9 & 26 & -28292 & 2940 \\
\hline $355 x-50$ & 89 & 10 & 29 & -30749 & 2910 \\
\hline
\end{tabular}


III. MAVAL PETROLEUM REserve MO. 1 HATER INJECTION SUMAMARIES 1989

\begin{tabular}{|c|c|c|c|c|c|}
\hline WELL/SECT & YR & MONTH & $\begin{array}{l}\text { DAYS ON } \\
\text { INJECTION }\end{array}$ & $\begin{array}{l}\text { HATER } \\
\text { INJECTION }\end{array}$ & $\begin{array}{l}\text { TUBIHG } \\
\text { PRESSURE }\end{array}$ \\
\hline $355 x-56$ & 89 & 11 & 30 & .32486 & 2990 \\
\hline $355 x-56$ & 89 & 12 & 30 & -31415 & 2972 \\
\hline $366 x-50$ & 89 & 1 & 31 & -21281 & 3013 \\
\hline $366 x-56$ & 89 & 2 & 28 & -16264 & 2986 \\
\hline $366 x-56$ & 89 & 3 & 31 & -18834 & 2997 \\
\hline $366 x-56$ & 89 & 4 & 30 & -18113 & 3000 \\
\hline $366 x-56$ & 89 & 5 & 31 & -17627 & 2997 \\
\hline $366 x-56$ & 89 & 6 & 30 & -15797 & 3000 \\
\hline $366 x-56$ & 89 & 7 & 31 & -16160 & 2992 \\
\hline $366 x-56$ & 89 & 8 & 31 & -18028 & 3000 \\
\hline $366 x-56$ & 89 & 9 & 26 & -15715 & 2992 \\
\hline $366 x-56$ & 89 & 10 & 31 & -17357 & 2927 \\
\hline $366 x-56$ & 89 & 11 & 30 & -18008 & 2997 \\
\hline $366 x-56$ & 89 & 12 & 30 & -18445 & 2998 \\
\hline $375-5 G$ & 89 & 1 & 31 & -53502 & 3005 \\
\hline $375-5 a$ & 89 & 2 & 28 & -43940 & 3018 \\
\hline $375-50$ & 89 & 3 & 31 & -48485 & 3010 \\
\hline $375-56$ & 89 & 4 & 30 & -44818 & 3000 \\
\hline $375-56$ & 89 & 5 & 31 & -47038 & 3003 \\
\hline $375-56$ & 89 & 6 & 30 & -43655 & 3000 \\
\hline $375-5 G$ & RO & 7 & 31 & -41755 & 3000 \\
\hline $3 \pi 5-50$ & 89 & 8 & 31 & -43246 & 2989 \\
\hline $375-56$ & 89 & 9 & 26 & -35859 & 3013 \\
\hline $375-56$ & 89 & 10 & 31 & -37136 & 2918 \\
\hline $375-56$ & 89 & 11 & 30 & -37951 & 2980 \\
\hline $375-56$ & 89 & 12 & 30 & -38148 & 2997 \\
\hline $386-56$ & 89 & 1 & 39 & -32356 & 3006 \\
\hline $386-56$ & 89 & 2 & 28 & -26607 & 2982 \\
\hline $386-5 G$ & 89 & 3 & 31 & -29698 & 3008 \\
\hline $386-5 G$ & 89 & 4 & 30 & -26765 & 2992 \\
\hline 386.56 & 89 & 5 & 31 & -28146 & 2998 \\
\hline $386-50$ & 89 & 6 & 30 & -27459 & 2992 \\
\hline $386-5 G$ & 89 & $?$ & 31 & -27414 & 3000 \\
\hline $386-5 G$ & 89 & 8 & 31 & -27106 & 2945 \\
\hline $386-5 c$ & 89 & 9 & 26 & -22717 & 2954 \\
\hline $386-5 G$ & 89 & 10 & 31 & -25586 & 2889 \\
\hline $386-56$ & 89 & 11 & 30 & -28014 & 2972 \\
\hline $386-5 G$ & 89 & 12. & 30 & -30006 & 3000 \\
\hline $342 A-6 a$ & 89 & 1 & 31 & -34582 & 2995 \\
\hline $342 A-6 G$ & 89 & 2 & 28 & -30681 & 3000 \\
\hline $342 A-66$ & 89 & 3 & 31 & -33807 & 3000 \\
\hline $342 A-6 G$ & 89 & 4 & 30 & -31467 & 3000 \\
\hline $342 A-6 G$ & 89 & 5 & 31 & -31727 & 2997 \\
\hline $34,2 A-6 G$ & 89 & 6 & 30 & -29721 & 3023 \\
\hline $342 A-66$ & 89 & 7 & 31 & -29537 & 3003 \\
\hline $342 A-66$ & 89 & 8 & 31 & -29077 & 3023 \\
\hline
\end{tabular}


III. MAVAL PETROLELM RESERVE MO. 1 WATER INJECTION SUMMARIES 1989

\begin{tabular}{|c|c|c|c|c|c|}
\hline MELL/SECT & YR & MONTH & $\begin{array}{l}\text { DAYS ON } \\
\text { INJECTION }\end{array}$ & $\begin{array}{c}\text { WATER } \\
\text { INJECTION }\end{array}$ & $\begin{array}{l}\text { TUBING } \\
\text { PRESSURE }\end{array}$ \\
\hline $342 A-6 G$ & 89 & 9 & 26 & -25193 & 3002 \\
\hline $342 A-66$ & 89 & 10 & 26 & -21310 & 2878 \\
\hline $342 A-6 G$ & 89 & 11 & 29 & -27640 & 2757 \\
\hline $342 A-66$ & 89 & 12 & 30 & -28892 & 2977 \\
\hline $363-66$ & 89 & 1 & 31 & -49600 & 3005 \\
\hline $363-60$ & 89 & 2 & 28 & -43025 & 3009 \\
\hline $363-66$ & 89 & 3 & 31 & .47979 & 3002 \\
\hline $363-66$ & 89 & 4 & 30 & -45312 & 3002 \\
\hline $363-60$ & 89 & 5 & 31 & -46407 & 2998 \\
\hline $363-60$ & 89 & 6 & 30 & -44690 & 3000 \\
\hline $363-60$ & 89 & 7 & 31 & -44251 & 2498 \\
\hline $363-60$ & 89 & 8 & 31 & -44911 & 3011 \\
\hline $363-60$ & 89 & 9 & 26 & -39741 & 2990 \\
\hline $363-66$ & 89 & 10 & 31 & -42948 & 2900 \\
\hline $363-60$ & 89 & 11 & 30 & -43026 & 2983 \\
\hline $363-66$ & 89 & 12 & 30 & -42821 & 2985 \\
\hline $384-66$ & 89 & 1 & 31 & -83690 & 3003 \\
\hline $384-60$ & 89 & 2 & 28 & .66028 & 3025 \\
\hline $384-66$ & 89 & 3 & 31 & -68504 & 3006 \\
\hline $384-66$ & 89 & 4 & 30 & -63324 & 3003 \\
\hline $384-60$ & 89 & 5 & 31 & -62981 & 2992 \\
\hline $384-66$ & 89 & 6 & 30 & -60916 & 2998 \\
\hline $384-66$ & 89 & 7 & 31 & -58813 & 2982 \\
\hline $384-60$ & 89 & 8 & 31 & -59318 & 3005 \\
\hline $384-60$ & 89 & 9 & 26 & -50140 & 2935 \\
\hline $384-66$ & 89 & 10 & 31 & -57108 & 2898 \\
\hline $384-6 G$ & 89 & 11 & 30 & -58379 & 2993 \\
\hline $384-66$ & 89 & 12 & 30 & -58343 & 2973 \\
\hline $21-15 c$ & 89 & 1 & 0 & 0 & 0 \\
\hline $21-150$ & 89 & 2 & 0 & 0 & 0 \\
\hline $21-156$ & 89 & 3 & 0 & 0 & 0 \\
\hline $21-15 a$ & 89 & 4 & 0 & 0 & 0 \\
\hline $29-156$ & 89 & 5 & 20 & -33498 & 137 \\
\hline $21-15 G$ & 89 & 6 & 30 & -42609 & 90 \\
\hline $21-15 G$ & 89 & 7 & 31 & -45025 & 98 \\
\hline $21-15 q$ & 89 & 8 & 31 & -47003 & 119 \\
\hline $29-156$ & 89 & 9 & 30 & -45147 & 0 \\
\hline $21-156$ & 89 & 10 & 31 & -42616 & 0 \\
\hline $21-856$ & 89 & 11 & 30 & -43381 & 0 \\
\hline $21-156$ & 89 & 12 & 29 & -62093 & 0 \\
\hline $42-160$ & 89 & 1 & 31 & -17264 & 105 \\
\hline $42-166$ & 89 & 2 & 23 & -23605 & 49 \\
\hline $42-166$ & 89 & 3 & 31 & -22433 & 70 \\
\hline $42-166$ & 89 & 4 & 30 & -21523 & 78 \\
\hline $42-166$ & 89 & 5 & 30 & -25623 & 60 \\
\hline $42 \cdot 166$ & 89 & 6 & 30 & -21257 & 72 \\
\hline
\end{tabular}


111. MAVAL PETROLEL RESERVE NO. 1 WATER INJECTION SUMURIES 1989

\begin{tabular}{|c|c|c|c|c|c|}
\hline WELL/SECT & YR & MONTH & $\begin{array}{l}\text { DAYS ON } \\
\text { INJECTION }\end{array}$ & $\begin{array}{c}\text { WATER } \\
\text { INJECTION }\end{array}$ & $\begin{array}{l}\text { TUBING } \\
\text { PRESSURE }\end{array}$ \\
\hline $42-166$ & 89 & 7 & 31 & -17579 & 0 \\
\hline $42-166$ & 89 & 8 & 31 & -18211 & 0 \\
\hline $42-160$ & 89 & 9 & 30 & -17030 & 124 \\
\hline $42-160$ & 89 & 10 & 31 & -21090 & 131 \\
\hline $42-166$ & 89 & 11 & 30 & -20881 & 124 \\
\hline $42-166$ & 89 & 12 & 29 & -22191 & 139 \\
\hline $324 A-7 R$ & 89 & 1 & 31 & -45629 & 1732 \\
\hline $324 A-7 R$ & 89 & 2 & 28 & -39665 & 1732 \\
\hline $324 A-7 R$ & 89 & 3 & 31 & -43980 & 1721 \\
\hline $324 A-7 R$ & 89 & 4 & 30 & -43185 & 1753 \\
\hline $324 A-7 R$ & 89 & 5 & 31 & -44531 & 1787 \\
\hline $324 A \cdot 7 R$ & 89 & 6 & 30 & -39926 & 1762 \\
\hline $324 A-7 R$ & 89 & 7 & 31 & -48551 & 1840 \\
\hline $324 A-7 R$ & 89 & 8 & 31 & -44689 & 1774 \\
\hline $324 A-7 R$ & 89 & 9 & 30 & -42377 & 1748 \\
\hline $324 A-7 R$ & 89 & 10 & 31 & -45266 & 1671 \\
\hline $326 A-7 R$ & 89 & 11 & 30 & -40292 & 1697 \\
\hline $324 A-7 R$ & 89 & 12 & 31 & -44144 & 1652 \\
\hline $333 A-7 R$ & 89 & 1 & 31 & -35752 & 0 \\
\hline $333 A-7 R$ & 89 & 2 & 28 & -31626 & 0 \\
\hline $333 A-7 R$ & 89 & 3 & 31 & -36678 & 200 \\
\hline $333 A-T R$ & 89 & 4 & 30 & -35401 & 1700 \\
\hline $333 A-7 R$ & 89 & 5 & 31 & $-37: 92$ & 433 \\
\hline $333 A-7 R$ & 89 & 6 & 30 & -31827 & 444 \\
\hline $333 A-7 R$ & 89 & 7 & 31 & -34444 & 487 \\
\hline $333 A-7 R$ & 69 & 8 & 31 & -35043 & 492 \\
\hline $333 A-7 R$ & 89 & 9 & 29 & -33521 & 515 \\
\hline $333 A-7 R$ & 89 & 10 & 29 & -32083 & 416 \\
\hline $333 A-7 R$ & 89 & 11 & 30 & -34124 & 569 \\
\hline $335 A-7 R$ & 89 & 12 & 31 & -37012 & 569 \\
\hline $337 A-7 R$ & 89 & 1 & 31 & -57476 & 248 \\
\hline $337 A-7 R$ & 89 & 2 & 28 & -60262 & 638 \\
\hline $337 A-7 R$ & 89 & 3 & 31 & -73811 & 1284 \\
\hline $337 A-7 R$ & 89 & 4 & 30 & -67060 & 1462 \\
\hline $337 A-7 R$ & 89 & 5 & 31 & -60058 & 1210 \\
\hline $337 A-7 R$ & 89 & 6 & 30 & -48126 & 361 \\
\hline $337 A-7 R$ & 89 & 7 & 31 & -52495 & 274 \\
\hline $337 A-7 R$ & 89 & 8 & 31 & -53143 & 282 \\
\hline $337 A-7 R$ & 89 & 9 & 30 & -58834 & 482 \\
\hline $337 A-7 R$ & 89 & 10 & 31 & -57212 & 405 \\
\hline $337 A-7 R$ & 89 & 11 & 30 & -56465 & 466 \\
\hline $337 A-7 R$ & 89 & 12 & 31 & $-64,921$ & 529 \\
\hline $3484-7 R$ & 89 & 1 & 31 & -54771 & 1984 \\
\hline $348 A-7 R$ & 89 & 2 & 28 & -58023 & 2025 \\
\hline $348 A-7 R$ & 89 & 3 & 31 & -68188 & 2113 \\
\hline $348 A-7 R$ & 89 & 4 & 30 & -60599 & 2168 \\
\hline
\end{tabular}


iII. MAVAL PETROLEUM RESERVE nO. 1 HATER INJECTION SUMLARIES 1989

\begin{tabular}{|c|c|c|c|c|c|}
\hline WELL/SECT & YR & MONTH & $\begin{array}{l}\text { DAYS OW } \\
\text { INJECTIOW }\end{array}$ & $\begin{array}{l}\text { WATER } \\
\text { INJECTION }\end{array}$ & $\begin{array}{l}\text { TUBING } \\
\text { PRESSURE }\end{array}$ \\
\hline $348 A-7 R$ & 89 & 5 & 31 & -62584 & 2139 \\
\hline $348 A-7 R$ & 89 & 6 & 30 & -47679 & 2025 \\
\hline $348 A-7 R$ & 89 & 7 & 31 & -52366 & 2097 \\
\hline $368 M-7 R$ & 89 & 8 & 31 & -51893 & 2055 \\
\hline $348 A-7 R$ & 89 & 9 & 30 & -47306 & 2055 \\
\hline $348 n-7 R$ & 89 & 10 & 31 & -50402 & 2071 \\
\hline $348 A-7 R$ & 89 & 11 & 30 & -45962 & 1938 \\
\hline $348 A-7 R$ & 89 & 12 & 31 & -50419 & 1985 \\
\hline $373 A-7 R$ & 89 & 1 & 31 & -77653 & 2511 \\
\hline $373 A-7 R$ & 89 & 2 & 28 & -65216 & 2593 \\
\hline $373 A-7 R$ & 89 & 3 & 30 & -72093 & 2554 \\
\hline $373 A-7 R$ & 89 & 4 & 30 & -72528 & 2367 \\
\hline $373 A-7 R$ & 89 & 5 & 31 & -78066 & 2327 \\
\hline $373 A-7 R$ & 89 & 6 & 30 & -67150 & 2164 \\
\hline $373 A-7 R$ & 89 & 7 & 31 & -73828 & 2173 \\
\hline $373 A-7 R$ & 89 & 8 & 31 & -66858 & 1952 \\
\hline $373 A-7 R$ & 89 & 9 & 30 & -65251 & 1960 \\
\hline $373 A-7 R$ & 89 & 10 & 29 & -68100 & 1912 \\
\hline $373 A-7 R$ & 89 & 11 & 30 & -65515 & 1652 \\
\hline $373 A-7 R$ & 89 & 12 & 31 & -72360 & 1526 \\
\hline $384 x-7 R$ & 89 & 1 & 31 & -61721 & 756 \\
\hline $384 x-7 R$ & 89 & 2 & 28 & -53647 & 823 \\
\hline $384 x-7 R$ & 89 & 3 & 31 & -64969 & 1195 \\
\hline $384 x-7 R$ & 89 & 4 & 30 & -64685 & 1365 \\
\hline $384 x-7 R$ & 89 & 5 & 31 & -64593 & 1556 \\
\hline $384 x-7 R$ & 89 & 6 & 30 & -54845 & 1398 \\
\hline $384 x-7 R$ & 89 & 7 & 31 & -63308 & 1708 \\
\hline $384 x-7 R$ & 89 & 8 & 31 & -57102 & 1527 \\
\hline $384 x-7 R$ & 89 & 9 & 30 & -48132 & 1445 \\
\hline $384 x-7 R$ & 89 & 10 & 31 & -56084 & 1466 \\
\hline $384 x-7 R$ & 89 & 11 & 30 & -53596 & 1519 \\
\hline $384 x-7 R$ & 89 & 12 & 31 & -59063 & 1526 \\
\hline $313-8 R$ & 89 & 1 & 31 & -69650 & 1489 \\
\hline $313-8 R$ & 89 & 2 & 28 & -63610 & 1507 \\
\hline $313-8 R$ & 89 & 3 & 31 & -74425 & 1552 \\
\hline $313-8 R$ & 89 & 4 & 30 & -72380 & 1637 \\
\hline $313-8 R$ & 89 & 5 & 31 & .73757 & 1671 \\
\hline $313-8 R$ & 89 & 6 & 30 & -64439 & $\uparrow 598$ \\
\hline $313-8 R$ & 89 & 7 & 31 & -74380 & 1642 \\
\hline $313-8 R$ & 89 & 8 & 31 & -68160 & 1565 \\
\hline $313-8 R$ & 89 & 9 & 30 & -66084 & 1585 \\
\hline $313-82$ & 89 & 10 & 31 & -71046 & 1616 \\
\hline $313-8 R$ & 89 & 11 & 30 & -66951 & 9536 \\
\hline $313-8 R$ & 89 & 12 & 30 & -75477 & 1539 \\
\hline $344-8 R$ & 89 & 1 & 31 & -459216 & 1856 \\
\hline $344-8 R$ & 89 & 2 & 28 & -39843 & 1980 \\
\hline
\end{tabular}


III. NAVAL PETROLEUM RESERVE NO. 1 WATER INJECTION SUMUARIES 1989

\begin{tabular}{|c|c|c|c|c|c|}
\hline WELL/SECT & YR & MONTH & $\begin{array}{l}\text { DAYS ON } \\
\text { INJECTION }\end{array}$ & $\begin{array}{l}\text { WATER } \\
\text { INJECTION }\end{array}$ & $\begin{array}{l}\text { TUBING } \\
\text { PRESSURE }\end{array}$ \\
\hline $344-8 R$ & 89 & 3 & 31 & -46228 & 1824 \\
\hline $344-8 R$ & 89 & 4 & 30 & -45187 & 1897 \\
\hline $344-8 R$ & 89 & 5 & 31 & -47368 & 1947 \\
\hline $344-8 R$ & 89 & 6 & 30 & -41077 & 1893 \\
\hline $344-8 R$ & 89 & 7 & 31 & -43883 & 1998 \\
\hline $344-8 R$ & 89 & 8 & 31 & -43795 & 1982 \\
\hline $344-8 R$ & 89 & 9 & 30 & -42854 & 1973 \\
\hline $344-8 R$ & 89 & 10 & 31 & -45467 & 1961 \\
\hline $344-8 R$ & 89 & 11 & 30 & -40924 & 1978 \\
\hline $344-8 R$ & 89 & 12 & 31 & -45647 & 1974 \\
\hline $364-8 R$ & 89 & 1 & 31 & -31927 & 2758 \\
\hline $364-8 R$ & 89 & 2 & 28 & -25791 & 2795 \\
\hline $364-8 R$ & 89 & 3 & 31 & -23953 & 2758 \\
\hline $364-8 R$ & B? & 4 & 30 & -26508 & 2740 \\
\hline $364-8 R$ & 89 & 5 & 31 & -20376 & 2566 \\
\hline $364-8 R$ & 89 & 6 & 30 & -18924 & 2584 \\
\hline $364-8 R$ & 89 & 7 & 31 & -18461 & 2653 \\
\hline $364-8 R$ & 89 & 8 & 31 & -24899 & 2926 \\
\hline $364-8 R$ & 89 & 9 & 30 & -27498 & 3063 \\
\hline $364-8 R$ & 89 & 10 & 31 & -26146 & 2848 \\
\hline $364-8 R$ & 89 & 11 & 29 & -16306 & 2638 \\
\hline $364-8 R$ & 89 & 12 & 31 & -18368 & 2555 \\
\hline $375-8 R$ & 89 & 9 & 31 & -8832 & 2702 \\
\hline $375-8 R$ & 89 & 2 & 28 & -7205 & 2796 \\
\hline $375-8 R$ & 89 & 3 & 31 & -8231 & 2877 \\
\hline $375-8 R$ & 89 & 4 & 30 & -11763 & 2888 \\
\hline $375-8 R$ & 89 & 5 & 31 & -12999 & 2763 \\
\hline $375-8 R$ & 89 & 6 & 30 & -8642 & 1993 \\
\hline $375-81$ & 89 & 7 & 28 & -8869 & 2066 \\
\hline $375-8 R$ & 89 & 8 & 29 & -13352 & 2559 \\
\hline $375-8 R$ & 89 & 9 & 21 & -7231 & 2207 \\
\hline $375-8 R$ & 89 & 10 & 31 & -15742 & 2739 \\
\hline $375-8 R$ & 89 & 11 & 30 & -14629 & 2381 \\
\hline $375-8 R$ & 89 & 12 & 31 & -15938 & 2406 \\
\hline $313 A-17 R$ & 89 & 1 & 0 & 0 & 2015 \\
\hline $313 A-17 R$ & 89 & 2 & 10 & -1246 & 2767 \\
\hline $313 A-17 R$ & 89 & 3 & 31 & -4694 & 2790 \\
\hline $313 A-17 R$ & 89 & 4 & 30 & -4552 & 2800 \\
\hline $313 A-17 R$ & 89 & 5 & 31 & -3606 & 2661 \\
\hline $313 A-17 R$ & 89 & 6 & 30 & -3628 & 2631 \\
\hline $313 A-17 R$ & 89 & 7 & 31 & -4488 & 2660 \\
\hline $313 A-17 R$ & 89 & 8 & 12 & -1577 & 2627 \\
\hline $313 A-17 R$ & 89 & 9 & 0 & 0 & 2627 \\
\hline $313 A-17 R$ & 89 & 10 & 0 & 0 & 2627 \\
\hline $313 A-17 R$ & 89 & 11 & 0 & 0 & 2627 \\
\hline $313 A-17 R$ & 89 & 12 & 0 & 0 & 2627 \\
\hline
\end{tabular}


III. MAVAL PETROLEL RESERVE NO. 1 WATER INJECTION SUMAARIES 1989

\begin{tabular}{|c|c|c|c|c|c|}
\hline WELL/SECT & YR & MONTH & $\begin{array}{l}\text { DAYS ON } \\
\text { INJECTION }\end{array}$ & $\begin{array}{l}\text { WATER } \\
\text { INJECTION }\end{array}$ & $\begin{array}{l}\text { TUBING } \\
\text { PRESSURE }\end{array}$ \\
\hline $333 A-17 R$ & 89 & 1 & 39 & -27692 & 2677 \\
\hline $333 A-17 R$ & 89 & 2 & 28 & -13645 & 2700 \\
\hline $333 A-17 R$ & 89 & 3 & 31 & -18143 & 2745 \\
\hline $333 A-17 R$ & 89 & 4 & 30 & -16897 & 2730 \\
\hline $333 A-17 R$ & 89 & 5 & 31 & -16222 & 2595 \\
\hline $333 A-17 R$ & 89 & 6 & 30 & -14995 & 2540 \\
\hline $333 A-17 R$ & 89 & 7 & 31 & -15787 & 2616 \\
\hline $333 A-17 R$ & 89 & 8 & 31 & -17617 & 2837 \\
\hline $333 A-17 R$ & 89 & 9 & 30 & -26149 & 2628 \\
\hline $333 A-97 R$ & 89 & 10 & 31 & -26550 & 1863 \\
\hline $333 A-17 R$ & 89 & 11 & 30 & -32946 & 2267 \\
\hline $333 A-17 R$ & 89 & 12 & 31 & ,36365 & 2463 \\
\hline $354 x-17 R$ & 89 & 1 & 28 & -11585 & 2646 \\
\hline $354 x-17 ?$ & 89 & 2 & 17 & " 1329 & 2765 \\
\hline $354 x-17 R$ & 89 & 3 & 31 & -1807 & 2800 \\
\hline $354 x-17 R$ & 89 & 4 & 30 & -2992 & 2823 \\
\hline $354 x-17 R$ & 89 & 5 & 31 & -1049 & 2668 \\
\hline $354 x-17 R$ & 89 & 6 & 30 & -843 & 2626 \\
\hline $354 x-17 R$ & 89 & 7 & 31 & -890 & 2647 \\
\hline $354 x-17 R$ & 89 & 8 & 31 & -1461 & 2882 \\
\hline $354 x-17 R$ & 89 & 9 & 30 & -1408 & 3030 \\
\hline $354 x-17 R$ & 89 & 10 & 30 & -1217 & 2861 \\
\hline $354 x-17 R$ & 89 & 11 & 22 & -373 & 2622 \\
\hline $354 x-172$ & 89 & 12 & 30 & -382 & 2527 \\
\hline $375 x-17 R$ & 89 & 1 & 0 & 0 & 2600 \\
\hline $375 x-17 R$ & 89 & 2 & 0 & 0 & 2600 \\
\hline $375 x-17 k$ & 89 & 3 & 0 & 0 & 2600 \\
\hline $375 x-172$ & 89 & 4 & 0 & 0 & 2600 \\
\hline $375 x-17 R$ & 89 & 5 & 0 & 0 & 2600 \\
\hline $375 x-17 k$ & 89 & 6 & 0 & 0 & 2600 \\
\hline $375 x-172$ & 89 & 7 & 0 & 0 & 2600 \\
\hline $375 x-17 R$ & 89 & 8 & 0 & 0 & 2600 \\
\hline $375 x-17 R$ & 89 & 9 & 0 & 0 & 2600 \\
\hline $375 x-172$ & 89 & 10 & 0 & 0 & 2600 \\
\hline $375 x-17 R$ & 89 & 11 & 0 & 0 & 2600 \\
\hline $375 x-17 R$ & 89 & 12 & 0 & 0 & 2600 \\
\hline $386-17 R$ & 89 & 1 & 0 & 0 & 2950 \\
\hline $386-17 R$ & 89 & 2 & 0 & 0 & 2950 \\
\hline $386-17 R$ & 89 & 3 & 0 & 0 & 2950 \\
\hline $386-17 R$ & 89 & 4 & 0 & 0 & 2950 \\
\hline $386-17 k$ & 89 & 5 & 0 & 0 & 2950 \\
\hline $386-17 R$ & 89 & 6 & 0 & 0 & 2950 \\
\hline $386-17 n$ & 89 & 7 & 0 & 0 & 2950 \\
\hline $386-17 R$ & 89 & 8 & 0 & 0 & 2950 \\
\hline $386-17 R$ & 89 & 9 & 0 & 0 & 2950 \\
\hline $386-17 R$ & 89 & 10 & 0 & 0 & 2950 \\
\hline
\end{tabular}


111. NAVAL PETROLEUM RESERVE NO. 1 WATER INJECTION SUMMARIES 1989

\begin{tabular}{|c|c|c|c|c|c|}
\hline WELL/SECT & YR & MONTH & $\begin{array}{l}\text { DAYS ON } \\
\text { INJECTIOW }\end{array}$ & $\begin{array}{c}\text { MATER } \\
\text { INJECTION }\end{array}$ & $\begin{array}{l}\text { TUBING } \\
\text { PRESSURE }\end{array}$ \\
\hline $386-17 R$ & 89 & 11 & 0 & 0 & 2950 \\
\hline $386-17 R$ & 89 & 12 & a & 0 & 2950 \\
\hline $318-18 R$ & 89 & 1 & 31 & -64053 & 2111 \\
\hline $318-18 R$ & 89 & 2 & 28 & -55773 & 2102 \\
\hline $318-18 R$ & 89 & 3 & 31 & -67168 & 2155 \\
\hline $318-18 R$ & 89 & 4 & 30 & -61653 & 2152 \\
\hline $318-18 R$ & 89 & 5 & 31 & -62494 & 2149 \\
\hline $318-18 R$ & 89 & 6 & 30 & -60876 & 2137 \\
\hline $318-18 R$ & 89 & 7 & 31 & -60607 & 2100 \\
\hline $318-18 n$ & 89 & 8 & 31 & -61151 & 2134 \\
\hline $318-18 R$ & 89 & 9 & 30 & -59719 & 2217 \\
\hline $318-18 R$ & 89 & 10 & 31 & -58478 & 2134 \\
\hline $318-18 R$ & 89 & 11 & 30 & -53423 & 2151 \\
\hline $398-18 R$ & 89 & 12 & 31 & -5371.9 & 2147 \\
\hline $351 A-18 R$ & 89 & 1 & 30 & -44296 & 4998 \\
\hline $351 A-18 R$ & 89 & 2 & 28 & -40969 & 2009 \\
\hline $351 A-18 R$ & 89 & 3 & 31 & -49371 & 2176 \\
\hline $351 A-18 R$ & 89 & 4 & $3 u$ & -49518 & 2342 \\
\hline $351 A-18 R$ & 89 & 5 & 31 & -53231 & 22.48 \\
\hline $351 A-18 R$ & 89 & 6 & 30 & -39022 & 2098 \\
\hline $351 A-18 R$ & 89 & 7 & 31 & -42509 & 2123 \\
\hline $351 A-18 R$ & 89 & 8 & 31 & -41523 & 2197 \\
\hline $351 A-18 R$ & 89 & 9 & 30 & -39987 & 2132 \\
\hline $351 A-18 R$ & 89 & 10 & 31 & -35352 & 1906 \\
\hline $351 A-18 R$ & 80 & 11 & 30 & -35487 & 1947 \\
\hline $351 A-18 R$ & 89 & 12 & 17 & -23284 & 2003 \\
\hline $362 A-18 R$ & 89 & 1 & 31 & -24994 & 2711 \\
\hline $362 A-18 R$ & 89 & 2 & 23 & -22862 & 2739 \\
\hline $362 A-18 R$ & 89 & 3 & 31 & -25033 & 2695 \\
\hline $362 A-18 R$ & 89 & 4 & 30 & -23974 & 2773 \\
\hline $362 A-98 R$ & 89 & 5 & 31 & -22496 & 2619 \\
\hline $362 A-18 R$ & 89 & 6 & 30 & -19360 & 2581 \\
\hline $362 A-18 R$ & 89 & 7 & 31 & -18842 & 2645 \\
\hline $362 A-18 R$ & 89 & 8 & 39 & -21676 & 2876 \\
\hline $362 A-18 R$ & 89 & 9 & 30 & -20684 & 2913 \\
\hline $362 A-18 R$ & 89 & 10 & 31 & -20288 & 2787 \\
\hline $362 A-18 R$ & 89 & 19 & 30 & -14235 & 2595 \\
\hline $362 A-18 R$ & 89 & 12 & 31 & -13567 & 2532 \\
\hline $382 A-18 R$ & 89 & 1 & 31 & -25825 & 1024 \\
\hline $382 A-18 R$ & 89 & 2 & 28 & -21928 & 1156 \\
\hline $382 A-18 R$ & 89 & 3 & 31 & -25800 & 1548 \\
\hline $382 A-18 R$ & 89 & 4 & 30 & -25145 & 1883 \\
\hline $382 A-18 R$ & 89 & 5 & 31 & -27519 & 2532 \\
\hline $382 A-18 R$ & 89 & 6 & 30 & -22173 & 2412 \\
\hline $382 A-18 R$ & 89 & 7 & 31 & -24137 & 2571 \\
\hline $382 A-18 R$ & 89 & 8 & 31 & -24589 & 2698 \\
\hline
\end{tabular}


III. WAVAL PETROLEY Reserve wo. 1 WATER INJECTION SUMAARIES 1989

\begin{tabular}{|c|c|c|c|c|c|}
\hline MELL/SECY & YR & MONTH & $\begin{array}{l}\text { DAYS ON } \\
\text { INJECTIOH }\end{array}$ & $\begin{array}{l}\text { WATER } \\
\text { INJECTION }\end{array}$ & $\begin{array}{l}\text { TUBING } \\
\text { PRESSURE }\end{array}$ \\
\hline $382 A-18 n$ & 89 & 9 & 30 & -21983 & 2767 \\
\hline $382 A-18 R$ & 89 & 10 & 31 & -24975 & 2698 \\
\hline $382 A-18 R$ & 89 & 11 & 30 & -22946 & 2500 \\
\hline $382 A-18 R$ & 89 & 12 & 30 & -22280 & 2445 \\
\hline $313 A-19 R$ & 89 & 1 & 31 & -102553 & 1509 \\
\hline $313 A-19 R$ & 89 & 2 & 28 & -94999 & 1650 \\
\hline $313 M-19 R$ & 89 & 3 & 31 & -105789 & 1631 \\
\hline $313 M-19 n$ & 89 & 4 & 30 & -100588 & 1600 \\
\hline $313 A-19 k$ & 89 & 5 & 31 & -105386 & 1640 \\
\hline $313 A-19 R$ & 89 & 6 & 30 & -109229 & 1733 \\
\hline $313 A-19 k$ & 89 & 7 & 31 & -111422 & 1811 \\
\hline $313 A-19 R$ & 89 & 8 & 39 & -119412 & 1840 \\
\hline $313 k-19 k$ & 89 & 9 & 30 & -104140 & 1770 \\
\hline $343 A-19 R$ & 89 & 10 & 31 & -122115 & 1703 \\
\hline $313 n-19 k$ & 89 & 11 & 30 & -103816 & 1787 \\
\hline $313 A-19 R$ & 89 & 12 & 39 & -116107 & 1855 \\
\hline $327-23 R$ & 89 & 1 & 31 & $-1261+1$ & 2998 \\
\hline $327-23 R$ & 89 & 2 & 28 & -11181 & 3018 \\
\hline $327-23 R$ & 89 & 3 & 31 & -11833 & 3006 \\
\hline $327-23 R$ & 89 & 4 & 30 & -41003 & 3000 \\
\hline $327-23 R$ & 89 & 5 & 31 & -11270 & 3003 \\
\hline $327-23 R$ & $\theta$ & 6 & 30 & -10535 & 3003 \\
\hline $327-23 n$ & 89 & 7 & 39 & -10068 & 2997 \\
\hline $327-23 R$ & 89 & 8 & 31 & -10335 & 2997 \\
\hline $327-232$ & 89 & 9 & 26 & .9128 & $2 m:$ \\
\hline $327-23 n$ & 89 & 10 & 31 & -10335 & 280 \\
\hline $327-23 R$ & 89 & 11 & 29 & -10124 & 2981 \\
\hline $327-23 k$ & 89 & 12 & 30 & -90816 & 2985 \\
\hline $3364-23 R$ & 80 & 1 & 31 & -25842 & 2995 \\
\hline $3364-23 R$ & 09 & 2 & 28 & -25763 & 3002 \\
\hline $3364-23 n$ & 89 & 3 & 31 & -27304 & 3002 \\
\hline $3364-23 R$ & 89 & 4 & 30 & -26386 & 3018 \\
\hline $336 A-23 R$ & 89 & 5 & 31 & -29245 & 3245 \\
\hline $336 A-23 R$ & 89 & 6 & 30 & -25501 & 3003 \\
\hline $336 A-23 R$ & 89 & 7 & 31 & -24687 & 2908 \\
\hline $336 A-23 R$ & 69 & 8 & 39 & -25291 & 3003 \\
\hline $3364-23 R$ & 89 & 9 & 26 & -23713 & 2996 \\
\hline $336 A-23 R$ & 89 & 10 & 31 & -23252 & 2908 \\
\hline $336 A-23 R$ & $\infty 8$ & 11 & 29 & -23525 & 2998 \\
\hline $336 A-23 n$ & 89 & 12 & 30 & -25696 & 2993 \\
\hline $366 x-23 x$ & 89 & $i$ & 31 & 24399 & 2987 \\
\hline $366 x-232$ & 89 & 2 & 28 & -23180 & 3000 \\
\hline $366 k-23 k$ & 89 & 3 & 31 & -24713 & 2989 \\
\hline $366 x-23 x$ & 39 & 4 & 30 & -23187 & 3000 \\
\hline $366 x-23 k$ & 89 & 5 & 31 & -.23503 & 3029 \\
\hline $366 x-23 R$ & 89 & 6 & 30 & -20912 & 3010 \\
\hline
\end{tabular}


111. MAVAl Petroleum reserve no. 1 WATER IHJECTION SUMARIES 1989

\begin{tabular}{|c|c|c|c|c|c|}
\hline WELL/SECT & YR & MONTH & $\begin{array}{l}\text { DAYS ON } \\
\text { INJECTION }\end{array}$ & $\begin{array}{l}\text { WATER } \\
\text { IMJECTION }\end{array}$ & $\begin{array}{l}\text { TUBING } \\
\text { PRESSURE }\end{array}$ \\
\hline $366 x-23 R$ & 89 & 7 & 31 & -20341 & 3000 \\
\hline $366 x-23 R$ & 89 & 8 & 31 & -20706 & 3000 \\
\hline $366 x-238$ & 89 & 9 & 26 & -18098 & 2981 \\
\hline $366 x-23 R$ & 89 & 10 & 25 & -75184 & 2898 \\
\hline $366 x-23 R$ & 89 & 11 & 28 & -200333 & 2857 \\
\hline $366 x-236$ & 89 & 12 & 30 & -22241 & 3002 \\
\hline $316-26 k$ & 89 & 1 & 0 & 0 & 0 \\
\hline $316-24 R$ & 89 & 2 & 0 & 0 & 0 \\
\hline $316-24 R$ & 89 & 3 & 0 & 0 & 0 \\
\hline $316-24 R$ & 89 & 4 & 0 & 0 & 0 \\
\hline $316-24 R$ & 89 & 5 & 0 & 0 & 0 \\
\hline $316-24 R$ & 89 & 6 & 0 & 0 & 0 \\
\hline $316-24 R$ & 89 & 7 & 0 & 0 & 0 \\
\hline $316-24 R$ & 89 & $B$ & 0 & 0 & 0 \\
\hline $316-24 R$ & 89 & 9 & 0 & 0 & 0 \\
\hline $316-24 R$ & 89 & 10 & 0 & 0 & 0 \\
\hline $316-24 R$ & 89 & 11 & 0 & 0 & 0 \\
\hline $316-24 R$ & 89 & 12 & 30 & -9560 & 2988 \\
\hline $316 A-24 R$ & 89 & 1 & 31 & -6540 & 3013 \\
\hline $316 A-24 R$ & 89 & 2 & 28 & -5698 & 3025 \\
\hline $3164-24 R$ & 89 & 3 & 31 & -5852 & 3019 \\
\hline $3164-24 R$ & 89 & 4 & 30 & -5774 & 2960 \\
\hline $316 A-24 R$ & 89 & 5 & 31 & $-5 \mathrm{rr}$ & 3008 \\
\hline $316 A-24 R$ & 89 & 6 & 30 & -5423 & 2998 \\
\hline $316 A-24 R$ & 89 & 7 & 31 & $-54,100$ & 2998 \\
\hline $316 A-24 R$ & 89 & 8 & 31 & -5472 & 3000 \\
\hline $316 A-24 R$ & 89 & 9 & 26 & -4504 & 2998 \\
\hline $316 A-24 R$ & 89 & 10 & 39 & -5184 & 2866 \\
\hline $316 A-24 R$ & 89 & 11 & 29 & -6236 & 2833 \\
\hline $3164-24 R$ & 89 & 12 & 50 & -8103 & 2925 \\
\hline $326-24 R$ & 89 & 1 & 31 & -1179 & 3900 \\
\hline $326-24 R$ & 89 & 2 & 28 & -9712 & 3079 \\
\hline $326-24 R$ & 89 & 3 & 31 & -8927 & 3079 \\
\hline $326-24 R$ & 89 & 4 & 30 & -7934 & 3100 \\
\hline $326-24 R$ & 89 & 5 & 31 & -7073 & 3081 \\
\hline $326-24 R$ & 89 & 6 & 30 & -5804 & 3902 \\
\hline $326-24 R$ & 89 & 7 & 31 & -5025 & 3910 \\
\hline $326-24 R$ & 89 & 8 & 31 & -5017 & 3100 \\
\hline $326-24 R$ & 89 & 9 & 26 & -3410 & 3071 \\
\hline $326-24 R$ & 89 & 10 & 24 & -2890 & 2867 \\
\hline $526-248$ & 89 & 11 & 29 & -13785 & 2897 \\
\hline $326-24 k$ & 89 & 12 & 30 & -11290 & 3050 \\
\hline $337 x-24 R$ & 89 & 1 & 31 & -8966 & 2963 \\
\hline $337 x-24 R$ & 89 & 2 & 28 & -8351 & 2971 \\
\hline $337 x-24 R$ & 89 & 3 & 31 & -.9053 & 3005 \\
\hline $337 x-26 R$ & 89 & 4 & 30 & -8267 & 3002 \\
\hline
\end{tabular}


III. Naval Petroleum Reserve Mo. 1 WATER INJECTION SUMARIES 1989

\begin{tabular}{|c|c|c|c|c|c|}
\hline WELL/SECT & YR & MONTH & $\begin{array}{l}\text { DAYS OH } \\
\text { INAECTION }\end{array}$ & $\begin{array}{c}\text { MATER } \\
\text { INJECTION }\end{array}$ & $\begin{array}{l}\text { RUBING } \\
\text { PRESSURE }\end{array}$ \\
\hline 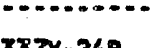 & $\cdots$ & 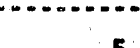 & ... & 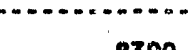 & 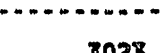 \\
\hline $337 x-26 R$ & 89 & 5 & 31 & -8300 & 3023 \\
\hline $337 x-24 R$ & 89 & 6 & 20 & -8233 & 3065 \\
\hline $337 x-24 R$ & 89 & 7 & 31 & -9847 & 3029 \\
\hline $337 x-24 R$ & 89 & 8 & 31 & -8865 & 3006 \\
\hline $337 x-24 R$ & 89 & 9 & 26 & -7708 & 2996 \\
\hline $337 x-248$ & 89 & 10 & 31 & -7878 & 2906 \\
\hline $337 x-262$ & 89 & 11 & 22 & -6757 & 2868 \\
\hline $337 x-24 R$ & 89 & 12 & 30 & -9003 & 3015 \\
\hline $348 x-248$ & 89 & 1 & 31 & -15671 & 2990 \\
\hline $348 x-24 R$ & 89 & 2 & 28 & -13470 & 2989 \\
\hline $348 K-24 R$ & 89 & 3 & 31 & -15381 & 2998 \\
\hline $348 x-24 R$ & 89 & 4 & 30 & -15139 & 2998 \\
\hline $348 x-248$ & 89 & 5 & 31 & -14978 & 2950 \\
\hline $348 x-24 R$ & 89 & 6 & 30 & -14391 & 2967 \\
\hline $348 x-24 R$ & 89 & 7 & 31 & -16420 & 2995 \\
\hline $348 x-24 R$ & 89 & 8 & 31 & $.147 r 9$ & 3000 \\
\hline $348 x-24 R$ & 89 & 9 & 26 & -12436 & 2956 \\
\hline $368 x-26 n$ & 89 & 10 & 31 & -13813 & 2900 \\
\hline $348 x-24 R$ & 89 & 11 & 29 & -13652 & 2998 \\
\hline $348 x-24 R$ & 89 & 12 & 30 & -14133 & 2970 \\
\hline $318-258$ & 89 & 1 & 31 & -24644 & 2974 \\
\hline $318-25 R$ & 89 & 2 & 28 & -21984 & 2989 \\
\hline $318-25 R$ & 80 & 3 & 31 & $-24,089$ & 2995 \\
\hline $318-25 k$ & 89 & 4 & 30 & -22210 & 2998 \\
\hline $318-25 R$ & 89 & 5 & 31 & .23349 & 2985 \\
\hline $318-258$ & 89 & 6 & 30 & -22363 & 3000 \\
\hline $318-25 R$ & 89 & 7 & 31 & -22305 & 2997 \\
\hline $318-25 R$ & 89 & 8 & 39 & -21966 & 2989 \\
\hline $318-25 R$ & 89 & 9 & 26 & - 1873 & 2973 \\
\hline $318-25 R$ & 89 & 10 & 31 & -21134 & 2884 \\
\hline $318-25 R$ & 89 & 11 & 30 & -19907 & 2935 \\
\hline $318-25 R$ & 89 & 12 & 30 & -19690 & 2905 \\
\hline $361 x-258$ & 89 & 1 & 31 & -111442 & 2904 \\
\hline $361 x-25 R$ & 89 & 2 & 28 & -10185 & 3000 \\
\hline $361 x-25 R$ & 89 & 3 & 31 & -112.24 & 3000 \\
\hline $361 x-25 R$ & 89 & 4 & 30 & -10616 & 2997 \\
\hline $361 x-25 R$ & 89 & 5 & 31 & -10745 & 2973 \\
\hline $361 x-25 R$ & 89 & 6 & 30 & -10259 & 3000 \\
\hline $361 x-25 R$ & 89 & 7 & 31 & -10158 & 2997 \\
\hline $369 x-25 R$ & 89 & 8 & $3 ?$ & -10462 & 3000 \\
\hline $361 x-25 R$ & 89 & 9 & 26 & -8923 & 2869 \\
\hline $361 x-25 R$ & 89 & 10 & 31 & -11522 & 2798 \\
\hline $361 x-25 R$ & 89 & 11 & 29 & -11197 & 2834 \\
\hline $361 x-25 R$ & 89 & 12 & 30 & -10967 & 3000 \\
\hline $379 x-25 R$ & 89 & 1 & 31 & -19541 & 3000 \\
\hline $379 x-25 R$ & 89 & 2 & 38 & -10534 & 3000 \\
\hline
\end{tabular}


1II. MAVAL PETROLEUM RESERVE MO. 1 HATER INJECTION SUMUARIES 1989

\begin{tabular}{|c|c|c|c|c|c|}
\hline MELL/SECT & $Y R$ & MONTH & $\begin{array}{l}\text { DAYS ON } \\
\text { INJECTION }\end{array}$ & $\begin{array}{c}\text { HATER } \\
\text { IAJECTION }\end{array}$ & $\begin{array}{l}\text { TUBING } \\
\text { PRESSURE }\end{array}$ \\
\hline $371 x-25 R$ & 89 & 3 & 31 & -11062 & 3003 \\
\hline $379 x-25 R$ & 88 & 4 & 30 & -10474 & 3000 \\
\hline $371 x-25 R$ & 89 & 5 & 31 & .9775 & 2995 \\
\hline $371 x-25 R$ & 89 & 6 & 30 & -10119 & 3000 \\
\hline $371 x-25 R$ & 89 & 7 & 31 & -9859 & 3000 \\
\hline $379 x-25 R$ & 89 & 8 & 31 & -10124 & 3000 \\
\hline $379 x-25 R$ & 89 & 9 & 26 & -8672 & 2985 \\
\hline $371 x-25 R$ & 89 & 10 & 31 & -9125 & 2919 \\
\hline $371 x-25 R$ & 89 & 11 & 29 & -9026 & 3000 \\
\hline $371 x-25 R$ & 89 & 12 & 30 & -9032 & 2958 \\
\hline $311-26 R$ & 89 & 1 & 31 & -31427 & 2995 \\
\hline $311-26 R$ & 89 & 2 & 28 & -28739 & 3002 \\
\hline $311-268$ & 89 & 3 & 31 & -31429 & 3003 \\
\hline $311-26 R$ & 89 & 4 & 30 & -28101 & 3005 \\
\hline $391-264$ & 89 & 5 & 31 & -27989 & 3005 \\
\hline $311-26 k$ & 89 & 6 & 29 & -24011 & 2975 \\
\hline $311-26 R$ & 89 & 7 & 31 & -28026 & 3000 \\
\hline $311-26 k$ & 89 & 8 & 31 & -28391 & 3000 \\
\hline $311-268$ & 89 & 9 & 26 & -24876 & 2979 \\
\hline $311-268$ & 89 & 10 & 31 & $-27 r 39$ & 2908 \\
\hline $3: 1-26 R$ & 89 & 11 & 23 & -24489 & 2780 \\
\hline $311 \cdot 260$ & 89 & 12 & 30 & 32780 & 2983 \\
\hline $322 A-26 A$ & 89 & 1 & 31 & -34504 & 2992 \\
\hline $322 A-26 R$ & 89 & 2 & 28 & -32609 & 2977 \\
\hline $322 A-26 R$ & 89 & 3 & 31 & -33916 & 3000 \\
\hline $322 A-26 n$ & 89 & 4 & 30 & -30858 & 3000 \\
\hline $322 A-26 R$ & 89 & 5 & 31 & -31062 & 2990 \\
\hline $322 A-26 A$ & 89 & 6 & 30 & -29279 & 2998 \\
\hline $322 A-26 R$ & 89 & 7 & 31 & -29271 & 2994 \\
\hline $322 A-26 R$ & 89 & 8 & 31 & -29622 & 3000 \\
\hline $322 A-26 R$ & 89 & 9 & 26 & -22806 & 2775 \\
\hline $322 A-26 R$ & 88 & 10 & 31 & -30142 & 2818 \\
\hline $322 A-26 n$ & 89 & 11 & 29 & -26325 & 296 \\
\hline $322 A-26 R$ & 89 & 12 & 30 & -26929 & 2962 \\
\hline $334 x-26 R$ & 89 & 1 & 31 & -20458 & 2094 \\
\hline $334 x-26 R$ & 89 & 2 & 28 & -17711 & 3000 \\
\hline $334 x+26 R$ & 89 & 3 & 31 & -19935 & 3000 \\
\hline $334 x-268$ & 89 & 6 & 30 & -18538 & 3003 \\
\hline $334 x-26 R$ & 89 & 5 & 31 & -18865 & 3000 \\
\hline $334 x-26 R$ & 89 & 6 & 30 & -18785 & 3000 \\
\hline $334 x-26 n$ & 89 & 7 & 31 & -18134 & 2908 \\
\hline $334 x-26 R$ & 89 & 8 & 31 & -18406 & 3000 \\
\hline $334 x-26 R$ & 89 & 9 & 26 & -16326 & 2962 \\
\hline $334 x-260$ & 89 & 10 & 31 & -18762 & 2402 \\
\hline $334 x-26 R$ & 89 & 11 & 29 & -17284 & 2979 \\
\hline $334 x-20 R$ & 89 & 12 & 30 & -18095 & 2967 \\
\hline
\end{tabular}


III. MAVAl PETroleun Reserve nO. 1 WATER INJECTION SUMMARIES 1989

\begin{tabular}{|c|c|c|c|c|c|}
\hline WELL/SECT & $Y R$ & MONTH & $\begin{array}{l}\text { DAYS ON } \\
\text { INJECTION }\end{array}$ & $\begin{array}{c}\text { HATER } \\
\text { INJECTION }\end{array}$ & $\begin{array}{c}\text { TUBING } \\
\text { PRESSURE }\end{array}$ \\
\hline $344 A-26 R$ & 89 & 1 & 31 & -9013 & 2994 \\
\hline $3 / 4 A-26 R$ & 89 & 2 & 28 & -8032 & 3000 \\
\hline $34+4-26 R$ & 89 & 3 & 31 & -8956 & 3000 \\
\hline $344 A-26 R$ & 89 & 4 & 30 & -8367 & 3005 \\
\hline $344 A-26 R$ & 89 & 5 & 31 & -8496 & 3002 \\
\hline $344 A-26 R$ & 89 & 6 & 30 & -8203 & 3000 \\
\hline $364 A-26 R$ & 89 & 7 & 31 & -8596 & 3000 \\
\hline $344 A-26 R$ & 89 & 8 & 31 & -8153 & 2997 \\
\hline $344 A-26 R$ & 89 & 9 & 26 & -7194 & 2954 \\
\hline $344 A-268$ & 89 & 10 & 31 & -7864 & 2905 \\
\hline $344 A-26 R$ & 89 & 11 & 29 & -7410 & 2978 \\
\hline $344 A-26 R$ & 89 & 12 & 30 & -7457 & 2942 \\
\hline $356 x-26 R$ & 89 & 1 & 31 & -4749 & 2998 \\
\hline $356 x-26 R$ & 89 & 2 & 28 & -4103 & 3000 \\
\hline $356 x-26 R$ & 89 & 3 & 31 & -3858 & 3003 \\
\hline $356 x-26$ & 89 & 4 & 30 & -3485 & 3000 \\
\hline $356 x-26 R$ & 89 & 5 & 31 & -4420 & 3000 \\
\hline $356 x-26 R$ & 89 & 6 & 30 & -4308 & 3000 \\
\hline $356 x-26 R$ & 89 & 7 & 31 & -3858 & 2998 \\
\hline $356 x-26 R$ & 89 & 8 & 31 & -3950 & 2997 \\
\hline $356 x-26 R$ & 89 & 9 & 26 & -3291 & 2987 \\
\hline $356 x-26 R$ & 89 & 10 & 25 & -6234 & 2567 \\
\hline $356 x-268$ & 89 & 14 & 30 & -101020 & 9962 \\
\hline $356 x-268$ & 89 & 12 & 12 & -36897 & 2418 \\
\hline $367-26 R$ & 89 & 1 & 31 & -75115 & 2989 \\
\hline $367-26 R$ & 89 & 2 & 28 & -64891 & 2952 \\
\hline $367-26 R$ & 89 & 3 & 31 & -78176 & 2990 \\
\hline $367-26 n$ & 89 & 4 & 30 & -77021 & 2997 \\
\hline $367-26 R$ & 89 & 5 & 31 & -87106 & 2982 \\
\hline $367-26 R$ & 89 & 6 & 30 & -57622 & 2982 \\
\hline $367-26 n$ & 89 & 7 & 31 & -56465 & 2997 \\
\hline $367-26 R$ & 89 & 8 & 31 & -6.9561 & 3000 \\
\hline $367-26 R$ & 89 & 9 & 26 & -47237 & 2971 \\
\hline $367-26 R$ & 89 & 10 & 31 & -58026 & 2897 \\
\hline $367-26 R$ & 89 & 11 & 24 & -42080 & 2837 \\
\hline $367-26 R$ & 89 & 12 & 30 & -55311 & 2942 \\
\hline $378-26 R$ & 89 & 1 & 0 & 0 & 0 \\
\hline $378-268$ & 89 & 2 & 0 & 0 & 0 \\
\hline $378-268$ & 89 & 3 & 0 & 0 & 0 \\
\hline $378-26 R$ & 89 & 4 & 0 & 0 & 0 \\
\hline $378-260$ & 89 & 5 & 0 & 0 & 0 \\
\hline $378-26 n$ & 89 & 6 & 0 & 0 & 0 \\
\hline $378-26 R$ & 89 & 7 & 0 & 0 & 0 \\
\hline $378-268$ & 89 & 8 & 0 & 0 & 0 \\
\hline $378-26 R$ & 89 & 9 & 0 & 0 & 0 \\
\hline $378-26 R$ & 89 & 10 & 0 & 0 & 0 \\
\hline
\end{tabular}


III. MAVAL PETROLELM RESERVE MO. 1 WATER INJECTION SUMARIES 1989

\begin{tabular}{|c|c|c|c|c|c|}
\hline WELL/SECT & YR & MONTH & $\begin{array}{l}\text { DAYS OH } \\
\text { INJECTIOA }\end{array}$ & $\begin{array}{c}\text { WATER } \\
\text { INJECTIOW }\end{array}$ & $\begin{array}{l}\text { TUBING } \\
\text { PRESSURE }\end{array}$ \\
\hline $378-26 R$ & 89 & 11 & 0 & 0 & 0 \\
\hline $378-26 R$ & 89 & 12 & 28 & -59193 & 2246 \\
\hline $378 x-26 R$ & 89 & 1 & 0 & 0 & 0 \\
\hline $378 x-26 R$ & 89 & 2 & 0 & 0 & 0 \\
\hline $378 x-268$ & 89 & 3 & 0 & 0 & 0 \\
\hline $378 x-26 R$ & 89 & 4 & 0 & 0 & 0 \\
\hline $378 x-26 R$ & 89 & 5 & 0 & 0 & 0 \\
\hline $378 x-26 R$ & 89 & 6 & 0 & 0 & 0 \\
\hline $378 x-26 R$ & 89 & 7 & 0 & 0 & 0 \\
\hline $378 x-26 R$ & 89 & 8 & 0 & 0 & 0 \\
\hline $378 x-26 R$ & 89 & 9 & 0 & 0 & 0 \\
\hline $378 x-268$ & 89 & 10 & 0 & 0 & 0 \\
\hline $378 x-268$ & 89 & 11 & 0 & 0 & 0 \\
\hline $378 x-26 R$ & 89 & 12 & 0 & 0 & 0 \\
\hline $321 x-36 R$ & 89 & $q$ & 31 & -80455 & 2876 \\
\hline $321 x-36 R$ & 89 & 2 & 29 & -70142 & 2895 \\
\hline $321 x-36 R$ & 89 & 3 & 31 & -75994 & 2979 \\
\hline $324 x-36 R$ & 89 & 4 & 30 & -69459 & 2950 \\
\hline $321 x-36 R$ & 89 & 5 & 31 & -67423 & 2931 \\
\hline $321 x-36 R$ & 89 & 6 & 30 & -62679 & 2987 \\
\hline $321 x-36 R$ & 89 & 7 & 31 & -58499 & 2955 \\
\hline $321 x-36 R$ & 89 & 8 & 31 & -56990 & 2974 \\
\hline $32.1 x-36 R$ & 89 & 9 & 26 & -39968 & 2523 \\
\hline $321 x-36 k$ & 89 & 10 & 31 & -55960 & 2824 \\
\hline $321 x-36 n$ & 89 & 11 & 30 & -52314 & 2880 \\
\hline $321 x-36 R$ & 89 & 12 & 30 & -51126 & 2888 \\
\hline $332 \times L-36 R$ & 89 & 1 & 31 & -25742 & 2952 \\
\hline $332 \times L-36 R$ & 89 & 2 & 28 & -22658 & 2970 \\
\hline $332 \times L-36 R$ & 89 & 3 & 31 & -25183 & 2987 \\
\hline $332 \times L-36 R$ & 89 & 4 & 30 & -23352 & 2985 \\
\hline $332 \times L-36 R$ & 89 & 5 & 31 & -23581 & 2977 \\
\hline $332 \times L-36 R$ & 89 & 6 & 30 & -22.422 & 2990 \\
\hline $332 X L-36 R$ & 89 & 7 & 31 & -22270 & 2976 \\
\hline $332 \times L-36 R$ & 89 & 8 & 31 & -21688 & 2976 \\
\hline $332 X L=36 R$ & 89 & 9 & 26 & -17944 & 2962 \\
\hline $332 \times L-36 R$ & 89 & 10 & 39 & -19732 & 2855 \\
\hline $33 \approx \times L-36 R$ & 89 & 19 & 30 & -19109 & 2918 \\
\hline $332 \times L-36 R$ & 89 & 12 & 30 & -19127 & 2887 \\
\hline $343 x-36 R$ & 89 & q & 31 & -54149 & 2905 \\
\hline $343 x-368$ & 89 & 2 & 28 & -48222 & 2932 \\
\hline $343 x-368$ & 89 & 3 & 31 & -48024 & 2984 \\
\hline $343 x-36 R$ & 89 & 4 & 30 & 42340 & 3000 \\
\hline $343 x-36 n$ & 89 & 5 & 31 & -40002 & 2989 \\
\hline $343 x-36 R$ & 89 & 6 & 30 & -36479 & 2997 \\
\hline $343 x-36 R$ & 89 & 7 & 31 & -36482 & 2995 \\
\hline $343 x-36 R$ & 89 & 8 & 31 & -36262 & 2992 \\
\hline
\end{tabular}


111. Maval PETroleum reserve no. 1

WATER INJECTION SUMARIES 1989

\begin{tabular}{|c|c|c|c|c|c|}
\hline WELL/SECT & YR & MONTH & $\begin{array}{l}\text { DAYS ON } \\
\text { INJECTION }\end{array}$ & $\begin{array}{l}\text { WATER } \\
\text { INJECTION }\end{array}$ & $\begin{array}{l}\text { TUBING } \\
\text { PRESSURE }\end{array}$ \\
\hline $343 x-36 R$ & 89 & 9 & 26 & -31275 & 2988 \\
\hline $343 x-36 R$ & 89 & 10 & 31 & -41469 & 2837 \\
\hline $343 x-36 R$ & 89 & 11 & 30 & -42060 & 2902 \\
\hline $343 x-36 R$ & 89 & 12 & 30 & -39854 & 2873 \\
\hline $355 x-36 h$ & 89 & 1 & 31 & -6926 & 2955 \\
\hline $355 x-368$ & 89 & 2 & 28 & -6175 & 2970 \\
\hline $355 x-36 R$ & 89 & 3 & 31 & -6834 & 2997 \\
\hline $355 x-36 R$ & 89 & 4 & 30 & $-659 n$ & 2993 \\
\hline $355 x-36 k$ & 39 & 5 & 31 & -6768 & 2987 \\
\hline $355 x-36 R$ & 89 & 6 & 30 & -6583 & 2997 \\
\hline $355 x-36 R$ & 89 & 7 & 13 & -4096 & 3000 \\
\hline $355 x-368$ & 89 & 8 & 27 & -81803 & 3050 \\
\hline $355 x-36 R$ & 89 & 9 & 4 & -1430 & 0 \\
\hline $355 x-36 R$ & 89 & 10 & 0 & 0 & 0 \\
\hline $355 x-36 R$ & 89 & 11 & 0 & 0 & 0 \\
\hline $355 x-36 R$ & 89 & 12 & 24 & -22408 & 1474 \\
\hline $366 x-36 R$ & 89 & 1 & 31 & -53428 & 2971 \\
\hline $366 x-36 R$ & 89 & 2 & 28 & -43929 & 2971 \\
\hline $366 x-36 R$ & 89 & 3 & 31 & -46766 & 2997 \\
\hline $366 x-36 R$ & 89 & 4 & 30 & -41851 & 3000 \\
\hline $366 x-36 R$ & 89 & 5 & 31 & -41399 & 2990 \\
\hline $366 x-368$ & 89 & 6 & 30 & -38647 & 3003 \\
\hline $366 x-36 R$ & 89 & 7 & 31 & -37262 & 2997 \\
\hline $366 x-36 R$ & 89 & 8 & 31 & -37137 & 3023 \\
\hline $366 x-36 R$ & 89 & 9 & 26 & -31660 & 2988 \\
\hline $366 x-36 R$ & 89 & 10 & 31 & -32955 & 2894 \\
\hline $366 x-36 R$ & 89 & 11 & 30 & -32671 & 2948 \\
\hline $366 x-36 R$ & 89 & 12 & 30 & -31395 & 2943 \\
\hline $376-36 R$ & 89 & 1 & 28 & -111901 & 2644 \\
\hline $376-36 R$ & 89 & 2 & 28 & -97254 & 2598 \\
\hline $376-36 R$ & 89 & 3 & 31 & -117702 & 2731 \\
\hline $376-36 R$ & 89 & 4 & 30 & -107774 & 2708 \\
\hline $376-361$ & 89 & 5 & 31 & -114879 & 2737 \\
\hline $376-36 R$ & 89 & 6 & 30 & -911576 & 2772 \\
\hline $376-36 R$ & 89 & 7 & 31 & $-10 / 516$ & 2797 \\
\hline $376-36 n$ & 89 & 8 & 31 & -101476 & 2598 \\
\hline $376-36 R$ & 89 & 9 & 26 & -74506 & 2394 \\
\hline $376-36 k$ & 89 & 10 & 39 & -95593 & 2534 \\
\hline $376-36 R$ & 89 & 11 & 30 & -97344 & 2668 \\
\hline $376-36 R$ & 89 & 12 & 30 & -94380 & 2615 \\
\hline $387-36 h$ & 89 & 1 & 31 & -10940 & 3005 \\
\hline $387-j$ & 89 & 2 & 28 & -9130 & 3009 \\
\hline $387-36$ & 89 & 3 & 31 & -9304 & 3006 \\
\hline $387-36 k$ & 89 & 4 & 30 & -7346 & 3002 \\
\hline $387-36 R$ & 89 & 5 & 31 & -7071 & 2997 \\
\hline $387-36 R$ & 89 & 6 & 30 & -6511 & 3000 \\
\hline
\end{tabular}


1II. MAVAL PETROLEUM RESERVE NO. 1 WATER INJECTION SUMUARIES 1989

\begin{tabular}{|c|c|c|c|c|c|}
\hline MELL/SECT & YR & MONTK & $\begin{array}{l}\text { DAYS ON } \\
\text { INJECTION }\end{array}$ & $\begin{array}{l}\text { WATER } \\
\text { INJECTION }\end{array}$ & $\begin{array}{l}\text { TUBING } \\
\text { PRESSURE }\end{array}$ \\
\hline $387-362$ & 89 & 7 & 31 & -6261 & 2997 \\
\hline $387-36 R$ & 89 & 8 & 31 & -6114 & 2998 \\
\hline $387-36 R$ & 89 & 9 & 26 & -4838 & 2998 \\
\hline $387 \cdot 36 R$ & 89 & 10 & 31 & -5435 & 2894 \\
\hline $387-36 R$ & 89 & 11 & 30 & .5089 & 2973 \\
\hline $387-36 R$ & 89 & 12 & 30 & -4462 & 2947 \\
\hline $318 L-29 S$ & 89 & 1 & 31 & -10358 & 3000 \\
\hline $318 L-29 S$ & 89 & 2 & 28 & -9541 & 3000 \\
\hline $318 L-29 S$ & 89 & 3 & 31 & -10504 & 3009 \\
\hline $318 L-29 S$ & 89 & 4 & 30 & -9558 & 2993 \\
\hline $318 L-29 S$ & 89 & 5 & 31 & -9665 & 2987 \\
\hline $318 \mathrm{~L}-29 \mathrm{~S}$ & 89 & 6 & 30 & -9785 & 2987 \\
\hline $318 L-295$ & 89 & 7 & 31 & -9821 & 2948 \\
\hline $318 L-29 S$ & 89 & 8 & 31 & -10781 & 2939 \\
\hline $318 \mathrm{~L}-29 \mathrm{~S}$ & 89 & 9 & 26 & -9140 & 2850 \\
\hline $318 L-295$ & 89 & 10 & 31 & -11602 & 2835 \\
\hline $318 L-29 S$ & 89 & 11 & 29 & -12014 & 2905 \\
\hline $318 L-29 S$ & 89 & 12 & 30 & -15010 & 2985 \\
\hline $313 x-305$ & 89 & 1 & 31 & -21684 & 3006 \\
\hline $313 x-305$ & 89 & 2 & 28 & -17523 & 2914 \\
\hline $313 x-305$ & 89 & 3 & 31 & -20691 & 3000 \\
\hline $313 x-30 s$ & 89 & 4 & 30 & -19714 & 3000 \\
\hline $313 x-305$ & 89 & 5 & 31 & -20345 & 2981 \\
\hline $313 x-305$ & 89 & 6 & 30 & -19806 & 3002 \\
\hline $313 x-305$ & 89 & 7 & 31 & -19702 & 2998 \\
\hline $313 x-30 s$ & 89 & 8 & 31 & -19842 & 3000 \\
\hline $313 x-30 s$ & 89 & 9 & 26 & -16962 & 2950 \\
\hline $313 x-305$ & 89 & 10 & 24 & -19470 & 2916 \\
\hline $313 x-30 s$ & 89 & 11 & 29 & -18514 & 2997 \\
\hline $313 x-30 s$ & 89 & 12 & 30 & -19544 & 2947 \\
\hline $323-30 s$ & 89 & 1 & 31 & -23684 & 3008 \\
\hline $323-305$ & 89 & 2 & 28 & -10229 & 3005 \\
\hline $323-30 s$ & 89 & 3 & 25 & -6098 & 3013 \\
\hline $323-305$ & 89 & 4 & 30 & -5727 & 3032 \\
\hline $323-30 s$ & 89 & 5 & 31 & -2820 & 2994 \\
\hline $323-305$ & 89 & 6 & 30 & -2341 & 3007 \\
\hline $323-30 s$ & 89 & 7 & 31 & -2298 & 2998 \\
\hline $323-30 s$ & 89 & 8 & 31 & -2514 & 3011 \\
\hline $323-305$ & 89 & 9 & 26 & -2174 & 3038 \\
\hline $323-30 s$ & 89 & 10 & 31 & -1712 & 2910 \\
\hline $323-305$ & 89 & 11 & 29 & -2074 & 2972 \\
\hline $323-305$ & 89 & 12 & 24 & -1621 & 2989 \\
\hline $324 \times L-30 S$ & 89 & 1 & 31 & -28575 & 3019 \\
\hline $324 \times L-305$ & 89 & 2 & 28 & -24923 & 3009 \\
\hline $324 X L-305$ & 89 & 3 & 31 & -26788 & 3003 \\
\hline $324 \times L-30 S$ & 89 & 4 & 30 & -24881 & 3002 \\
\hline
\end{tabular}


III. NAVAL PETROLEUM RESERVE nO. 1 WATER INJECTION SUMARIES 1989

\begin{tabular}{|c|c|c|c|c|c|}
\hline WELL/SECT & $Y R$ & MONTH & $\begin{array}{l}\text { DAYS ON } \\
\text { INJECTION }\end{array}$ & $\begin{array}{l}\text { WATER } \\
\text { IN,IECTION }\end{array}$ & $\begin{array}{l}\text { TUBING } \\
\text { PRESSURE }\end{array}$ \\
\hline $324 \times L-30 S$ & 89 & 5 & 31 & -24214 & 3000 \\
\hline $324 \times L-30 S$ & 89 & 6 & 30 & -23337 & 3000 \\
\hline $324 \times L-30 S$ & 89 & 7 & 31 & -22033 & 2998 \\
\hline $324 \times L-30 s$ & 89 & 8 & 31 & -22485 & 3000 \\
\hline $324 \times L-30 S$ & 89 & 9 & 26 & -18913 & 2990 \\
\hline $324 \times L-30 S$ & 89 & 10 & 31 & -19927 & 2929 \\
\hline $324 \times L-30 S$ & 89 & 11 & 29 & -19338 & 3000 \\
\hline $324 \times L-30 S$ & 89 & 12 & 30 & -19004 & 2997 \\
\hline $345 x-30 s$ & 89 & 1 & 31 & -25094 & 3103 \\
\hline $345 x-305$ & 89 & 2 & 28 & -23759 & 3089 \\
\hline $345 x-30 s$ & 89 & 3 & 31 & -24067 & 3089 \\
\hline $345 x-305$ & 89 & 4 & 30 & -21727 & 3113 \\
\hline $345 x-30 s$ & 89 & 5 & 31 & -22112 & 3092 \\
\hline $345 x-305$ & 89 & 6 & 30 & -20993 & 3098 \\
\hline $345 x-30 s$ & 89 & 7 & 31 & -20260 & 3058 \\
\hline $345 x-305$ & 89 & 8 & 31 & -21099 & 3123 \\
\hline $345 x-305$ & 89 & 9 & 26 & -18402 & 3102 \\
\hline $345 x-305$ & 89 & 10 & 31 & -18656 & 3002 \\
\hline $345 x-305$ & 89 & 11 & 30 & -19027 & 3071 \\
\hline $345 x-305$ & 89 & 12 & 30 & -20222 & 3078 \\
\hline $365-30 s$ & 89 & 1 & 31 & -32802 & 3002 \\
\hline $365 \cdot 305$ & 89 & 2 & 28 & -27636 & 3002 \\
\hline $365-30 s$ & 89 & 3 & 31 & -27515 & 3002 \\
\hline $365-30 s$ & 89 & 4 & 30 & .25394 & 3005 \\
\hline $365-30 s$ & 89 & 5 & 31 & -24809 & 2981 \\
\hline $365-30 s$ & 89 & 6 & 30 & -22756 & 3037 \\
\hline $365-305$ & 89 & 7 & 31 & -21605 & 3011 \\
\hline $365-30 s$ & 89 & 8 & 31 & -21084 & 3011 \\
\hline $365-305$ & 89 & 9 & 26 & -16574 & 3008 \\
\hline $365-305$ & 89 & 10 & 25 & -11716 & 2914 \\
\hline $365 \cdot 30 S$ & 89 & 11 & 28 & -14365 & 2898 \\
\hline $365-305$ & 89 & 12 & 30 & -14416 & 3008 \\
\hline $377-30 s$ & 89 & 1 & 31 & -18140 & 3100 \\
\hline $377 \cdot 30 s$ & 89 & 2 & 28 & -16518 & 3091 \\
\hline $377 \cdot 30 s$ & 89 & 3 & 31 & -17700 & 3089 \\
\hline $377-305$ & 89 & 4 & 30 & -18120 & 3097 \\
\hline $377-30 s$ & 89 & 5 & 31 & -19571 & 3077 \\
\hline $377-30 s$ & 89 & 6 & 30 & -18915 & 3097 \\
\hline $377-30 s$ & 89 & 7 & 31 & -18522 & 3092 \\
\hline $377-30 s$ & 89 & 8 & 31 & -19005 & 3082 \\
\hline 377-30s & 89 & 9 & 26 & -16964 & 3069 \\
\hline $377-30 s$ & 89 & 10 & 31 & -20935 & 2776 \\
\hline $377-305$ & 89 & 11 & 30 & -23387 & 2879 \\
\hline $377-30 s$ & 89 & 12 & 30 & -22848 & 2830 \\
\hline $3284-315$ & 89 & 1 & 31 & -93739 & 2973 \\
\hline $328 n-31 s$ & 89 & 2 & 28 & -82653 & 2982 \\
\hline
\end{tabular}


111. MAVAL PETROLELW RESERVE HO. 1 HATER INJECTION SUMAMARIES 1989

\begin{tabular}{|c|c|c|c|c|c|}
\hline WELL/SECT & YR & MONTH & $\begin{array}{l}\text { DAYS ON } \\
\text { INJECTION }\end{array}$ & $\begin{array}{c}\text { WATER } \\
\text { INJECTION }\end{array}$ & $\begin{array}{l}\text { TUBING } \\
\text { PRESSURE }\end{array}$ \\
\hline $3284-3$ is & 89 & 3 & 31 & -91430 & 2982 \\
\hline $328 A-31 s$ & 89 & 4 & 30 & -87343 & 2980 \\
\hline $3284-315$ & 89 & 5 & 31 & -88646 & 2981 \\
\hline $3284-315$ & 89 & 6 & 30 & -86030 & 2988 \\
\hline $328 A-315$ & 89 & 7 & 31 & -76995 & 2989 \\
\hline $328 n-315$ & 89 & 8 & 31 & -84419 & 2934 \\
\hline $328 n-315$ & 89 & 9 & 26 & -70322 & 2917 \\
\hline $3284-315$ & 89 & 10 & 31 & -77846 & 2874 \\
\hline $3284-315$ & 89 & 11 & 30 & -78656 & 2943 \\
\hline $328 A-315$ & 89 & 12 & 30 & -77251 & 2908 \\
\hline $331-325$ & 89 & 1 & 8 & -200 & 3032 \\
\hline $331-325$ & 89 & 2 & 0 & 0 & 3032 \\
\hline $331-32.5$ & 89 & 3 & 0 & 0 & 3032 \\
\hline $331-32 s$ & 89 & 4 & 0 & 0 & 3032 \\
\hline $331-325$ & 89 & 5 & 0 & 0 & 3032 \\
\hline $331-325$ & 89 & 6 & 0 & 0 & 3032 \\
\hline $531-325$ & 89 & 7 & 0 & 0 & 3032 \\
\hline $331-325$ & 89 & 8 & 0 & 0 & 3032 \\
\hline $331-325$ & 89 & 9 & 0 & 0 & 3032 \\
\hline $331-325$ & 89 & 10 & 0 & 0 & 3032 \\
\hline $331-325$ & 89 & 11 & 0 & 0 & 3032 \\
\hline $331-325$ & 89 & 12 & 0 & 0 & 3032 \\
\hline $333-32 s$ & 89 & 1 & 0 & 0 & 0 \\
\hline $333-325$ & 89 & 2 & 0 & 0 & 0 \\
\hline $333-32 s$ & 89 & 3 & 0 & 0 & 0 \\
\hline $333-325$ & 89 & 4 & 0 & 0 & 0 \\
\hline $333-325$ & 89 & 5 & 0 & 0 & 0 \\
\hline $333-325$ & 89 & 6 & 0 & 0 & 0 \\
\hline $333-325$ & 89 & 7 & 0 & 0 & 0 \\
\hline $333-325$ & 89 & 8 & 0 & 0 & 0 \\
\hline $333-325$ & 89 & 9 & 0 & 0 & 0 \\
\hline $333-32 s$ & 89 & 10 & 0 & 0 & 0 \\
\hline $333-32 s$ & 89 & 11 & 17 & -42329 & 2394 \\
\hline 333-32s & 89 & 12 & 30 & .61084 & 3000 \\
\hline $351 x-32 s$ & 89 & 1 & 0 & 0 & 3025 \\
\hline $351 x-325$ & 89 & 2 & 0 & 0 & 3025 \\
\hline $351 x-325$ & 89 & 3 & 0 & 0 & 3025 \\
\hline $351 x-325$ & 89 & 4 & 0 & 0 & 3025 \\
\hline $351 x-32 s$ & 89 & 5 & 0 & 0 & 3025 \\
\hline $351 x-325$ & 89 & 6 & 0 & 0 & 3025 \\
\hline $351 x-325$ & 89 & 7 & 0 & 0 & 3025 \\
\hline $351 x-325$ & 89 & 8 & 0 & 0 & 3025 \\
\hline $351 x-325$ & 89 & 9 & 0 & 0 & 3025 \\
\hline $351 x-325$ & 89 & 10 & 0 & 0 & 3025 \\
\hline $351 x-325$ & 89 & 19 & 0 & 0 & 3025 \\
\hline $351 x-3215$ & 89 & 12 & 0 & 0 & 3025 \\
\hline
\end{tabular}


III. Maval petrolelum reserve no. 1 WATER INJECTION SUMMARIES 1989

\begin{tabular}{|c|c|c|c|c|c|}
\hline WELL/SECT & YR & MONTH & $\begin{array}{l}\text { DAYS ON } \\
\text { INJECTICH }\end{array}$ & $\begin{array}{l}\text { WATER } \\
\text { INJECTION }\end{array}$ & $\begin{array}{l}\text { TUBING } \\
\text { PRESSURE }\end{array}$ \\
\hline $353-325$ & 89 & 1 & $\therefore$ & 0 & 0 \\
\hline $353-325$ & 89 & 2 & 0 & 0 & 0 \\
\hline 353-32S & 89 & 3 & 0 & 0 & 0 \\
\hline $353-325$ & 89 & 4 & 0 & 0 & 0 \\
\hline 353-32S & 89 & 5 & 0 & 0 & 0 \\
\hline $353-325$ & 89 & 6 & 0 & 0 & 0 \\
\hline 353-32S & 89 & 7 & 0 & 0 & 0 \\
\hline $353-325$ & 89 & 8 & 0 & 0 & 0 \\
\hline 353-32S & 89 & 9 & 0 & 0 & 0 \\
\hline 353-32S & 89 & 10 & 0 & 0 & 0 \\
\hline $353-325$ & 89 & 11 & 17 & -22639 & 2859 \\
\hline $353-325$ & 89 & 12 & 30 & -26483 & 2987 \\
\hline $373-325$ & 89 & 1 & 0 & $\because 0$ & 0 \\
\hline $373-325$ & 89 & 2 & 0 & 0 & 0 \\
\hline $373-325$ & 89 & 3 & 0 & $\because 0$ & 0 \\
\hline $373-325$ & 89 & 4 & 0 & 0 & 0 \\
\hline $373-325$ & 89 & 5 & 0 & 0 & 0 \\
\hline $373-325$ & 89 & 6 & 0 & 0 & 0 \\
\hline $373-325$ & 89 & 7 & 6 & -6407 & 3050 \\
\hline $373-325$ & 89 & 8 & 31 & -30569 & 3026 \\
\hline $373-325$ & 89 & 9 & 26 & -20481 & 2996 \\
\hline $373-325$ & 89 & 10 & 25 & -15819 & 2904 \\
\hline 373-325 & 89 & 11 & 29 & -18066 & 2857 \\
\hline $373-325$ & 89 & 12 & 30 & -18406 & 2982 \\
\hline 382-32s & 89 & 1 & 25 & -646 & 3106 \\
\hline 382-32S & 89 & 2 & 19 & -896 & 2936 \\
\hline $382-325$ & 89 & 3 & 31 & -1321 & 3102 \\
\hline $382-325$ & 89 & 4 & 29 & -1259 & 3098 \\
\hline 382-32S & 89 & 5 & 31 & -1259 & 3084 \\
\hline 382-32S & 89 & 6 & 30 & -1336 & 3180 \\
\hline 382-32s & 89 & 7 & 30 & -1134 & 3089 \\
\hline $382-325$ & 89 & 8 & 30 & -1203 & 3094 \\
\hline $382-325$ & 89 & 9 & 26 & -1065 & 3004 \\
\hline 382-325 & 89 & 10 & 28 & -1275 & 2985 \\
\hline 382-32S & 89 & 11 & 30 & -1305 & 3105 \\
\hline $382-325$ & 89 & 12 & 31 & -1212 & 3057 \\
\hline $314-335$ & 89 & 1 & 39 & -2748 & 2997 \\
\hline $314-33 S$ & 89 & 2 & 19 & -1413 & 2906 \\
\hline $314-335$ & 89 & 3 & 31 & -2299 & 3010 \\
\hline $314-335$ & 89 & 4 & 30 & -2032 & 3005 \\
\hline $314-335$ & 89 & 5 & 28 & -1932 & 3014 \\
\hline $314-335$ & 89 & 6 & 24 & -803 & 3085 \\
\hline $314-335$ & 89 & 7 & 15 & -915 & 3095 \\
\hline $314-335$ & 89 & 8 & 6 & -116 & 3100 \\
\hline $314-335$ & 89 & 9 & 15 & -562 & 3094 \\
\hline $314-335$ & 39 & 10 & 13 & -355 & 3031 \\
\hline
\end{tabular}


111. MAYAL PETROLEUM RESERVE Mo. 1 WATER INJECTION SLMMARIES 1989

\begin{tabular}{|c|c|c|c|c|c|}
\hline WELL/SECT & YR & MONTH & $\begin{array}{l}\text { DAYS ON } \\
\text { INJECTION }\end{array}$ & $\begin{array}{l}\text { WATER } \\
\text { INJECYION }\end{array}$ & $\begin{array}{c}\text { TUBING } \\
\text { PRESSURE }\end{array}$ \\
\hline $314 \cdot 335$ & 89 & 19 & 9 & -92 & 3089 \\
\hline $314-335$ & 89 & 12 & 9 & -199 & 3073 \\
\hline $333-335$ & 89 & 1 & 31 & -6884 & 2989 \\
\hline $333-33 s$ & 89 & 2 & 28 & -6173 & 3000 \\
\hline 333-33s & 89 & 3 & 31 & -6973 & 3000 \\
\hline $333-335$ & 89 & 4 & 30 & -7186 & 2997 \\
\hline $333-335$ & 89 & 5 & 31 & -7324 & 2998 \\
\hline $333-335$ & 89 & 6 & 30 & -7290 & 3008 \\
\hline 333-33s & 89 & 7 & 31 & -6948 & 3039 \\
\hline $333-33 s$ & 89 & 8 & 31 & -6985 & 3000 \\
\hline $333-33 s$ & 89 & 9 & 26 & -5820 & 2975 \\
\hline 333-33s & 89 & 10 & 31 & -6431 & 2916 \\
\hline $333-33 s$ & 89 & 11 & 24 & -5165 & 2743 \\
\hline $333-335$ & 89 & 12 & 30 & -6841 & 3015 \\
\hline $335-33 s$ & 89 & 1 & 31 & .5331 & 3074 \\
\hline $335-33 s$ & 89 & 2 & 28 & -4415 & 3105 \\
\hline $335-33 s$ & 89 & 3 & 31 & -4522 & 3024 \\
\hline $335-33 S$ & 89 & 4 & 30 & -3910 & 3042 \\
\hline $335-33 S$ & 89 & 5 & 31 & -3985 & 3081 \\
\hline $335-335$ & 89 & 6 & 30 & -3595 & 3068 \\
\hline $335-33 s$ & 89 & 7 & 31 & -3400 & 3003 \\
\hline $335-33 S$ & 89 & 8 & 31 & -3392 & 3052 \\
\hline $335-33 s$ & 89 & 9 & 26 & -3079 & 2965 \\
\hline $335-335$ & 89 & 10 & 31 & -3354 & 2961 \\
\hline $335-335$ & 89 & 11 & 30 & -3226 & 3065 \\
\hline $335-335$ & 89 & 12 & 30 & -3074 & 3033 \\
\hline $353-33 s$ & 89 & 1 & 31 & -3120 & 3006 \\
\hline $353-335$ & 89 & 2 & 28 & -2745 & 2980 \\
\hline $353-335$ & 89 & 3 & 31 & -3104 & 3000 \\
\hline $353-335$ & 89 & 4 & 30 & -2965 & 3000 \\
\hline $353-335$ & 89 & 5 & 31 & -3091 & 2997 \\
\hline $353-33 s$ & 89 & 6 & 30 & -3027 & 3000 \\
\hline $353-335$ & 89 & 7 & 31 & -3012 & 3019 \\
\hline $353-335$ & 89 & 8 & 31 & -2929 & 2990 \\
\hline $353-33 s$ & 89 & 9 & 25 & -2340 & 2900 \\
\hline $353-33 s$ & 89 & 10 & 31 & -2937 & 2919 \\
\hline $353-335$ & 89 & 11 & 30 & -2954 & 2997 \\
\hline $353-335$ & 89 & 12 & 30 & -2854 & 2952 \\
\hline $355-335$ & 89 & 1 & 31 & -17603 & 3002 \\
\hline $355-33 s$ & 89 & 2 & 28 & -15473 & 2989 \\
\hline $355-33 s$ & 89 & 3 & 39 & -16827 & 3015 \\
\hline $355-335$ & 89 & 4 & 30 & -15804 & 2988 \\
\hline $355-33 s$ & 89 & 5 & 31 & -16487 & 2969 \\
\hline $355-33 s$ & 189 & 6 & 30 & -16305 & 2978 \\
\hline $355-33 s$ & 89 & 7 & 31 & -16461 & 2968 \\
\hline $355-33 S$ & 89 & 8 & 31 & -17416 & 2994 \\
\hline
\end{tabular}


11. NAVAL PETROLEL RESERVE NO. 1 WATER INJECTION SUMMARIES 1989

\begin{tabular}{|c|c|c|c|c|c|}
\hline HELL/SECT & YR & MONTH & $\begin{array}{l}\text { DAYS ON } \\
\text { INJECTYON }\end{array}$ & $\begin{array}{c}\text { WATER } \\
\text { INJECTION }\end{array}$ & $\begin{array}{l}\text { TUBING } \\
\text { PRESSURE }\end{array}$ \\
\hline nats & $-\infty$ & 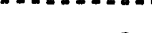 & 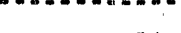 & ene & 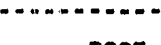 \\
\hline $355-335$ & 89 & 9 & 26 & -14328 & 2885 \\
\hline $355-33 s$ & 89 & 10 & 31 & -17248 & 2808 \\
\hline $355-33 s$ & 89 & 11 & 30 & -17348 & 2700 \\
\hline $355-33 s$ & 89 & 12 & 30 & -17138 & 2780 \\
\hline $363 x-335$ & 89 & 1 & 31 & -18964 & 3002 \\
\hline $363 x-335$ & 89 & 2 & 28 & -16939 & 3009 \\
\hline $363 x-335$ & 89 & 3 & 31 & -18686 & 3005 \\
\hline $363 x-33 s$ & 89 & 4 & 30 & -17824 & 3000 \\
\hline $363 x-335$ & 89 & 5 & 31 & -18305 & 2984 \\
\hline $363 x-335$ & 89 & 6 & 30 & -17899 & 3000 \\
\hline $363 x-33 s$ & 89 & 7 & 31 & -17741 & 3000 \\
\hline $363 x-33 s$ & 89 & 8 & 31 & -18145 & 3000 \\
\hline $363 x-335$ & 89 & 9 & 26 & -14924 & 2965 \\
\hline $363 x-335$ & 89 & 10 & 31 & -17737 & 2913 \\
\hline $363 x-335$ & 89 & 11 & 30 & -17395 & 2993 \\
\hline $363 x-335$ & 89 & 12 & 30 & -16990 & 2958 \\
\hline $375-33 s$ & 89 & 1 & 31 & -16922 & 3011 \\
\hline $375-33 s$ & 89 & 2 & 17 & -8698 & 3029 \\
\hline $375-33 s$ & 89 & 3 & 2 & -561 & 3029 \\
\hline $375-335$ & 89 & 4 & 0 & 0 & 3029 \\
\hline $375-33 s$ & 89 & 5 & 0 & 0 & 3029 \\
\hline $375-335$ & 89 & 6 & 0 & 0 & 3029 \\
\hline $375-335$ & 89 & 7 & 0 & 0 & 3029 \\
\hline $375-335$ & 89 & 8 & 0 & 0 & 3029 \\
\hline $375-33 s$ & 89 & 9 & 0 & 0 & 3029 \\
\hline $375-335$ & 89 & 10 & 0 & 0 & 3029 \\
\hline $375-335$ & 89 & 11 & 0 & 0 & 3029 \\
\hline $375-335$ & 89 & 12 & 0 & 0 & 3029 \\
\hline $382-335$ & 89 & 1 & 31 & -6097 & 3002 \\
\hline $382-335$ & 89 & 2 & 28 & -4546 & 3000 \\
\hline $382-33 s$ & 89 & 3 & 19 & -3374 & 3014 \\
\hline $382-335$ & 89 & 4 & 26 & .5565 & 3029 \\
\hline $382-335$ & 89 & 5 & 31 & -5280 & 3003 \\
\hline $382-335$ & 89 & 6 & 30 & -4936 & 3000 \\
\hline $382-335$ & 89 & 7 & 31 & -4938 & 3000 \\
\hline $382-335$ & 89 & 8 & 31 & -4732 & 2990 \\
\hline $382-33 s$ & 89 & 9 & 25 & -4011 & 2948 \\
\hline $382-335$ & 89 & 10 & 31 & -4827 & 2965 \\
\hline $382-335$ & 89 & 11 & 30 & -4658 & 3015 \\
\hline $382-335$ & 89 & 12 & 30 & -4514 & 2978 \\
\hline $315-34 \mathrm{~s}$ & 89 & 1 & 31 & -6590 & 3003 \\
\hline $315-345$ & 89 & 2 & 28 & -5925 & 3009 \\
\hline $315-3 / 45$ & 89 & 3 & 31 & -6480 & 2994 \\
\hline $315-34 \mathrm{~s}$ & 89 & 4 & 30 & -6206 & 3000 \\
\hline $315-345$ & 89 & 5 & 31 & -6474 & 2982 \\
\hline $315-345$ & 89 & 6 & 30 & -6362 & 3003 \\
\hline
\end{tabular}


III. MAVAL PETROLLUM RESERVE MO. 1 WATER INJECTION SUMMARIES 1989

\begin{tabular}{|c|c|c|c|c|c|}
\hline WELL/SECT & YR & MONTH & $\begin{array}{l}\text { DAYS ON } \\
\text { INJECTION }\end{array}$ & $\begin{array}{l}\text { WATER } \\
\text { INJECTION }\end{array}$ & $\begin{array}{l}\text { TUBING } \\
\text { PRESSURE }\end{array}$ \\
\hline $315-345$ & 89 & 7 & 31 & -6255 & 2998 \\
\hline $315-34 \mathrm{~s}$ & 89 & 8 & 31 & -6594 & 2987 \\
\hline $315-344 \mathrm{~S}$ & 89 & 9 & 26 & -5360 & 2948 \\
\hline $315-345$ & 89 & 10 & 31 & -6436 & 2923 \\
\hline $315-345$ & 89 & 11 & 30 & -6540 & 2993 \\
\hline $315-34 S$ & 89 & 12 & 30 & -6365 & 2938 \\
\hline $322-345$ & 89 & 1 & 31 & -8705 & 3000 \\
\hline $322-34 S$ & 89 & 2 & 28 & -7040 & 2998 \\
\hline $322-345$ & 89 & 3 & 31 & -7686 & 3010 \\
\hline $322-345$ & 89 & 4 & 30 & .7354 & 2995 \\
\hline $322-345$ & 89 & 5 & 31 & -7529 & 2994 \\
\hline $322-345$ & 89 & 6 & 30 & -7340 & 3005 \\
\hline $322-345$ & 89 & 7 & 31 & -7403 & 3000 \\
\hline $322-345$ & 89 & 8 & 31 & -7292 & 3013 \\
\hline $322-34 \mathrm{~s}$ & 89 & 9 & 26 & -6085 & 2977 \\
\hline $322-34 S$ & 89 & 10 & 31 & -6934 & 2924 \\
\hline $322-34 S$ & 89 & 19 & 30 & -7262 & 3000 \\
\hline $322-345$ & 89 & 12 & 30 & -7274 & 2983 \\
\hline $324-345$ & 89 & 1 & 31 & -16389 & 3002 \\
\hline $324-345$ & 89 & 2 & 28 & -14033 & 2998 \\
\hline $324-345$ & 89 & 3 & 31 & -15709 & 2998 \\
\hline $324-345$ & 89 & 4 & 30 & -14885 & 3000 \\
\hline $324-345$ & 89 & 5 & 31 & -14898 & 2994 \\
\hline $324-345$ & 89 & 6 & 30 & -13643 & 2932 \\
\hline $324-345$ & 89 & 7 & 31 & -14049 & 2990 \\
\hline $324-345$ & 89 & 8 & 31 & -14008 & 3000 \\
\hline $324-345$ & 89 & 9 & 26 & -11849 & 2942 \\
\hline $324-345$ & 89 & 10 & 31 & - $\hat{1} 3572$ & 2919 \\
\hline $324-345$ & 89 & 11 & 30 & -13693 & 3000 \\
\hline $324-34 \mathrm{~s}$ & 89 & 12 & 30 & -13503 & 2993 \\
\hline $326-345$ & 89 & 1 & 0 & 0 & 0 \\
\hline $326-34 \mathrm{~S}$ & 89 & 2 & 0 & 0 & 0 \\
\hline $326-345$ & 89 & 3 & 0 & 0 & 0 \\
\hline $326-345$ & 89 & 4 & 0 & 0 & 0 \\
\hline $326-345$ & 89 & 5 & 0 & 0 & 0 \\
\hline $326-345$ & 89 & 6 & 0 & ) & 0 \\
\hline $326-3445$ & 89 & 7 & 0 & 0 & 0 \\
\hline $326-345$ & 89 & 8 & 0 & 0 & 0 \\
\hline $326-345$ & 89 & 9 & 0 & 0 & 0 \\
\hline $326-345$ & 89 & 10 & 0 & 0 & 0 \\
\hline $326-345$ & 89 & 11 & 16 & -40982 & 1441 \\
\hline $326-345$ & 89 & 12 & 30 & -114631 & 2017 \\
\hline $335-34 \mathrm{~S}$ & 89 & 1 & 31 & -39825 & 3000 \\
\hline $335-345$ & 89 & 2 & 28 & -36017 & 3000 \\
\hline $335-34 S$ & 89 & 3 & 31 & -39609 & 3002 \\
\hline $335-34 \mathrm{~S}$ & 89 & 4 & 29 & -35863 & 2997 \\
\hline
\end{tabular}


III. MAVAL PETROLEUA RESERVE MO. 1

UATER IHJECTION SUMAARIES 1989

\begin{tabular}{|c|c|c|c|c|c|}
\hline WELL/SECT & $Y R$ & MONTH & $\begin{array}{l}\text { DAYS OW } \\
\text { IWJECTIOW }\end{array}$ & $\begin{array}{l}\text { MATER } \\
\text { IWJECTION }\end{array}$ & $\begin{array}{l}\text { TUBINA } \\
\text { PRESSURE }\end{array}$ \\
\hline $335-345$ & 89 & 5 & 31 & -38640 & 2997 \\
\hline $335-345$ & 89 & 6 & 30 & -37684 & 3012 \\
\hline $335-348$ & 84 & 7 & 31 & -36725 & 3000 \\
\hline 335: 145 & 89 & 8 & 31 & -37653 & 3000 \\
\hline $335-365$ & 80 & 9 & 26 & -31485 & 2981 \\
\hline $335-345$ & 89 & 10 & 31 & -36878 & 3774 \\
\hline $335-345$ & 89 & 11 & 30 & -37381 & 2999 \\
\hline $335-345$ & 89 & 12 & 30 & -34805 & 2903 \\
\hline $342-345$ & 89 & $\mathbf{i}$ & 31 & $-i 5 i z i$ & 3003 \\
\hline $342 \cdot 345$ & 89 & 2 & 28 & -12290 & 3009 \\
\hline $342-345$ & 89 & 3 & 27 & -11330 & 2996 \\
\hline $342-345$ & 89 & 4 & 30 & -15221 & 2965 \\
\hline $342-345$ & 89 & 5 & 31 & -13540 & 3011 \\
\hline $342-345$ & 89 & 6 & 30 & -12766 & 3015 \\
\hline $342-345$ & 89 & 7 & 31 & -12677 & 3015 \\
\hline $342-345$ & 89 & 8 & 31 & -13039 & 3026 \\
\hline 342.345 & 80 & 9 & 26 & -10438 & 3013 \\
\hline $342-345$ & 89 & 10 & 31 & -11771 & 2927 \\
\hline $342-345$ & 89 & 11 & 30 & -12421 & 2940 \\
\hline $342-345$ & 89 & 12 & 30 & -12335 & 2935 \\
\hline $346-345$ & 89 & 1 & 0 & 0 & 3150 \\
\hline $366-3 / 45$ & 89 & 2 & 0 & 0 & 3150 \\
\hline $344-345$ & 89 & 3 & 0 & 0 & 3150 \\
\hline $344-345$ & 89 & 4 & 0 & 0 & 3150 \\
\hline $344-345$ & 89 & 5 & 0 & 0 & 3150 \\
\hline $344-345$ & 89 & 6 & 0 & 0 & 3150 \\
\hline $364-365$ & 89 & 7 & 0 & 0 & 3150 \\
\hline $344-348$ & 89 & 8 & 0 & 0 & 3150 \\
\hline $344-345$ & 89 & 9 & 0 & 0 & 3150 \\
\hline $364-345$ & 89 & 10 & 0 & 0 & 3150 \\
\hline $344-345$ & 89 & 11 & 0 & 0 & 3150 \\
\hline $364-345$ & 89 & 12 & 0 & 0 & 3150 \\
\hline $355-343$ & 89 & 9 & 31 & -56902 & 3055 \\
\hline $355-34 \mathrm{~s}$ & 89 & 2 & $2 B$ & -51834 & 3088 \\
\hline $355-36,8$ & 89 & 3 & 31 & .57114 & 3095 \\
\hline $355-345$ & 89 & 4 & 30 & -53674 & 3093 \\
\hline $355-345$ & 89 & 5 & 31 & -54495 & 3063 \\
\hline $355-345$ & 89 & 6 & 30 & -53201 & 3100 \\
\hline $35.5-345$ & 89 & 7 & 31 & -52039 & 3115 \\
\hline $355-345$ & 89 & 8 & 31 & -53841 & 3100 \\
\hline $355-345$ & 89 & 9 & 25 & -39193 & 3087 \\
\hline $355-345$ & 89 & 10 & 31 & .50744 & 2930 \\
\hline $355-345$ & 80 & 11 & 30 & .5497 & 3002 \\
\hline $355-34 s$ & 89 & 12 & 30 & -52203 & 2095 \\
\hline $362-3465$ & 89 & 1 & 39 & -5189 & 2971 \\
\hline $362-345$ & 89 & 2 & 28 & -4907 & 3007 \\
\hline
\end{tabular}


11I. MAVAL PETROLEUM RESERVE MO. 1 MATER IHJECTION SUMARIES 1989

\begin{tabular}{|c|c|c|c|c|c|}
\hline WELLL/SECT & YR & MONTH & $\begin{array}{l}\text { DAYS ON } \\
\text { INJECTION }\end{array}$ & $\begin{array}{l}\text { MATER } \\
\text { INJECTYON }\end{array}$ & $\begin{array}{l}\text { TUBING } \\
\text { PRESSURE }\end{array}$ \\
\hline $36 i-345$ & 89 & 3 & 31 & .5295 & 3002 \\
\hline $362-345$ & 89 & 4 & 30 & -5002 & 3003 \\
\hline $362-348$ & 89 & 5 & 31 & -4992 & 2963 \\
\hline $362-345$ & 89 & 6 & 30 & -5172 & 3060 \\
\hline $362-345$ & 89 & 7 & 31 & -5188 & 3079 \\
\hline $362 \cdot 345$ & 89 & 8 & 31 & -4995 & 3071 \\
\hline $362-345$ & 89 & 9 & 26 & -4412 & 2969 \\
\hline $362-34 \$$ & 89 & 10 & 31 & -4289 & 2903 \\
\hline $36 \bar{z}-3 \overline{4} \hat{s}$ & $8 \overline{8}$ & ij & 30 & $-3 \pi 39$ & 2793 \\
\hline $362-345$ & 89 & 12 & 30 & -3391 & 2700 \\
\hline $373-345$ & 89 & 1 & 31 & -7936 & 3000 \\
\hline $373 \cdot 345$ & 89 & 2 & 28 & .7066 & 3009 \\
\hline $373-345$ & 89 & 3 & 31 & -7623 & 2992 \\
\hline $373-34 s$ & 89 & 4 & 30 & -7327 & 3000 \\
\hline $373-345$ & 89 & 5 & 30 & -7509 & 3017 \\
\hline $373-345$ & 89 & 6 & 29 & -6767 & 2869 \\
\hline $373 \cdot 345$ & 89 & 7 & 31 & -8111 & 3015 \\
\hline $373-345$ & 89 & 8 & 31 & -8338 & 3050 \\
\hline $373-345$ & 89 & 9 & 26 & -6275 & 2962 \\
\hline $373-345$ & 89 & 10 & 31 & -7187 & 2921 \\
\hline $373-345$ & 89 & 11 & 30 & -7738 & 3020 \\
\hline $373-345$ & 89 & 12 & 30 & -7473 & 2997 \\
\hline $375-345$ & 89 & 1 & 31 & -90747 & 3003 \\
\hline $375-345$ & 89 & 2 & 28 & -77611 & 3007 \\
\hline $375-345$ & 89 & 3 & 31 & -83856 & 3000 \\
\hline $375-345$ & 89 & 4 & 30 & -80410 & 3000 \\
\hline $375-345$ & 89 & 5 & 31 & -82267 & 2994 \\
\hline $375-345$ & 89 & 6 & 30 & -81876 & 3007 \\
\hline $375-345$ & 89 & 7 & 31 & -82494 & 3000 \\
\hline $3 / 5-345$ & 89 & 8 & 31 & -81397 & 3000 \\
\hline $375-345$ & 89 & 9 & 26 & -61058 & 2775 \\
\hline $375-345$ & 89 & 10 & 25 & -66478 & 2961 \\
\hline $3 / 5-345$ & 89 & 11 & 20 & -79265 & 2888 \\
\hline $375-345$ & 89 & 12 & 30 & -83173 & 3022 \\
\hline $313-355$ & 89 & 9 & $3 i$ & -9286 & 3002 \\
\hline $313-35 s$ & 89 & 2 & 28 & -8426 & 3000 \\
\hline $313 \cdots 355$ & 89 & 3 & 31 & -8640 & 3000 \\
\hline $313-35 s$ & 89 & 4 & 30 & -8467 & 3007 \\
\hline $313-35 s$ & 89 & 5 & 31 & -8739 & 3000 \\
\hline $313-35 s$ & 89 & 6 & 30 & -8053 & 2907 \\
\hline $313-35 s$ & 89 & 7 & 31 & -6660 & 2768 \\
\hline $313-35 s$ & 89 & 8 & 31 & -8566 & 2994 \\
\hline $313-35 s$ & 89 & 9 & 26 & -7156 & 3025 \\
\hline $313-355$ & 89 & 10 & 26 & -6978 & 2929 \\
\hline $313-35 s$ & 89 & $? 1$ & 15 & -4126 & 2820 \\
\hline $313-355$ & 89 & 12 & 30 & -8038 & 2975 \\
\hline
\end{tabular}


111. MAVAL PETROLEUM RESERVE No. 1 MATER INJECTION SUMMARIES 1989

\begin{tabular}{|c|c|c|c|c|c|}
\hline WELL/SECT & YR & MONTH & $\begin{array}{l}\text { DAYS ON } \\
\text { INJECTION }\end{array}$ & $\begin{array}{c}\text { WATER } \\
\text { IMJECTIION }\end{array}$ & $\begin{array}{l}\text { TUBIHG } \\
\text { PRESSURE }\end{array}$ \\
\hline $322-355$ & 89 & 1 & 31 & -7535 & 3081 \\
\hline $322-35 s$ & 89 & 2 & 28 & -7465 & 3082 \\
\hline $322-355$ & 89 & 3 & 31 & -7365 & 3076 \\
\hline $322-358$ & 89 & 4 & 30 & -7235 & 3097 \\
\hline $322-355$ & 89 & 5 & 31 & -7432 & 3024 \\
\hline $322-355$ & 89 & 6 & 30 & -7408 & 3095 \\
\hline $322-35 s$ & 89 & 7 & 31 & -7176 & 3082 \\
\hline $322-355$ & 89 & 8 & 31 & -7309 & 3097 \\
\hline $322-355$ & 89 & 9 & 206 & $-60,5$ & 3000 \\
\hline $322-355$ & 89 & 10 & 25 & -5422 & 3097 \\
\hline $322-355$ & 89 & 11 & 19 & -4638 & 2732 \\
\hline $322-35 s$ & 89 & 12 & 30 & -7346 & 3025 \\
\hline $342 x-35 s$ & 89 & 1 & 31 & -11395 & 3077 \\
\hline $342 x-355$ & 89 & 2 & 28 & -10593 & 3105 \\
\hline $362 x-35 s$ & 89 & 3 & 31 & -11337 & $30 \mathrm{Tt}$ \\
\hline $342 x-355$ & 89 & 4 & 30 & -10937 & 3093 \\
\hline $342 x-35 s$ & 89 & 5 & 31 & -11083 & 3029 \\
\hline $342 x-355$ & 89 & 6 & 30 & -11310 & 3143 \\
\hline $342 x-35 s$ & 89 & 7 & 31 & -10761 & 3900 \\
\hline $342 x-355$ & 89 & 8 & 39 & -10680 & 3085 \\
\hline $342 x-355$ & 89 & 9 & 26 & -8998 & 3069 \\
\hline $342 x-355$ & 89 & 10 & 25 & -7764 & 3029 \\
\hline $342 x-.35 s$ & 89 & 11 & 19 & -6569 & 2747 \\
\hline $342 x-355$ & 89 & 12 & 30 & -10587 & 2973 \\
\hline $363 x-35 s$ & 89 & i & 31 & -29263 & 3018 \\
\hline $363 x-35 s$ & 89 & 2 & 28 & -26286 & 3018 \\
\hline $363 x-35 s$ & 80 & 3 & 31 & -28056 & 3016 \\
\hline $363 x-35 s$ & 89 & 4 & 30 & -26084 & 3000 \\
\hline $363 x-35 s$ & 89 & 5 & 31 & -26828 & 3003 \\
\hline $363 x-355$ & 89 & 6 & 30 & -25332 & 2997 \\
\hline $363 x-35 s$ & 89 & 7 & 39 & -27057 & 3024 \\
\hline $363 x-355$ & 89 & 8 & 31 & -25356 & 2974 \\
\hline $363 x-35 s$ & 89 & 9 & 26 & -21402 & 2985 \\
\hline $363 x-35 s$ & 89 & 10 & 25 & -18934 & 2938 \\
\hline $363 x-35 s$ & 89 & 11 & 19 & -15798 & 2679 \\
\hline $363 x-35 s$ & 80 & 12 & 30 & -26964 & 2933 \\
\hline $364-355$ & 80 & 1 & 31 & -41049 & 3034 \\
\hline $364-355$ & 89 & 2 & 28 & -38625 & 3107 \\
\hline $354 \cdot 35 s$ & 89 & 3 & 31 & -39734 & 3085 \\
\hline $364-355$ & 89 & 4 & 30 & -37097 & 3083 \\
\hline $364_{1}-355$ & 89 & 5 & 31 & -36.899 & 3061 \\
\hline $364-358$ & 89 & 6 & 30 & -35223 & 3108 \\
\hline $364-355$ & 89 & 7 & 31 & -32634 & 3023 \\
\hline $364-.35 s$ & 89 & 8 & 31 & -33865 & 3048 \\
\hline $364-355$ & 89 & 9 & 26 & -28850 & 3092 \\
\hline 364.355 & 89 & 10 & 25 & -23782 & 3017 \\
\hline
\end{tabular}


11. MAVAL PETROLEUA RESERVE HO. 1 UATER INJECTION SUMUARIES 1989

\begin{tabular}{|c|c|c|c|c|c|}
\hline WELL/SECT & $Y R$ & MONTH & $\begin{array}{l}\text { DAYS ON } \\
\text { INJECTION }\end{array}$ & $\begin{array}{l}\text { WATER } \\
\text { INJECTION }\end{array}$ & $\begin{array}{l}\text { TUBING } \\
\text { PRESSURE }\end{array}$ \\
\hline $364-355$ & 89 & 11 & 19 & -17606 & 2508 \\
\hline $36 / 4-355$ & 89 & 12 & 30 & -33921 & 2940 \\
\hline $368-355$ & 89 & 1 & 31 & -56339 & 3005 \\
\hline $368-35 \%$ & 89 & 2 & 28 & -50211 & 3018 \\
\hline $368-355$ & 89 & 3 & 31 & -51568 & 3010 \\
\hline $368-35 s$ & 89 & 4 & 30 & -48184 & 3007 \\
\hline $368-355$ & 89 & 5 & 31 & -49061 & 3003 \\
\hline $368-35 S$ & 89 & 6 & 30 & -47264 & 3007 \\
\hline $368-355$ & 69 & 7 & 51 & $-4 \overline{7576}$ & 2998 \\
\hline $368-35 s$ & 89 & 8 & 39 & -49042 & 2998 \\
\hline $368-35 s$ & 89 & 9 & 26 & -39386 & 3021 \\
\hline $368-355$ & 89 & 10 & 25 & -35399 & 2931 \\
\hline $368 \cdot-35 s$ & 89 & 11 & 30 & -29178 & 2882 \\
\hline $368-35 s$ & 89 & 12 & 31 & -166791 & 2935 \\
\hline $374 x-35 s$ & 89 & 1 & 31 & -32742 & 3002 \\
\hline $374 x-355$ & 89 & 2 & 28 & -29674 & 3007 \\
\hline $374 x-355$ & 89 & 3 & 31 & -31194 & 3000 \\
\hline $374 x-355$ & 89 & 4 & 30 & -28697 & 3020 \\
\hline $374 x-35 s$ & 89 & 5 & 31 & -29488 & 3013 \\
\hline $374 x-35 s$ & 89 & 6 & 30 & -29700 & 3007 \\
\hline $374 x-355$ & 89 & 7 & 31 & -29317 & 3002 \\
\hline $374 x-35 s$ & 89 & 8 & 31 & -28330 & 3006 \\
\hline $374 x-35 s$ & 89 & 9 & 26 & -238600 & 2990 \\
\hline $374 x-35 s$ & 89 & 10 & 31 & -27590 & 2963 \\
\hline $374 x-35 s$ & 89 & 11 & 24 & -24020 & 2849 \\
\hline $374 x-35 s$ & 89 & 12 & 30 & -30439 & 2990 \\
\hline $3 \pi-35 s$ & 89 & 1 & 31 & -54389 & 2596 \\
\hline $375-35 s$ & 89 & 2 & 28 & -48084 & 2502 \\
\hline $375-355$ & 89 & 3 & 31 & -57070 & 2616 \\
\hline $375-355$ & 89 & 4 & 30 & -58856 & 2773 \\
\hline $375-35 s$ & 89 & 5 & 39 & -54726 & 2626 \\
\hline $375.35 s$ & 89 & 6 & 30 & -53349 & 2593 \\
\hline $375-35 s$ & 89 & 7 & 31 & -52435 & 2590 \\
\hline $375 \cdot 355$ & 89 & 8 & 31 & -55778 & 2658 \\
\hline $375-35 s$ & 89 & 9 & 26 & -41545 & 2.525 \\
\hline $375-355$ & 89 & 10 & 25 & -43240 & 2646 \\
\hline $375-35 s$ & 89 & 11 & 30 & -35102 & 2392 \\
\hline $375-35 s$ & 89 & 12 & 30 & -51765 & 2503 \\
\hline $377-35 s$ & 89 & 1 & 0 & 0 & 0 \\
\hline $377-35 s$ & 89 & 2 & 0 & 0 & 0 \\
\hline $377-35 s$ & 89 & 3 & 31 & -83009 & 2752 \\
\hline $377-355$ & 89 & 4 & 28 & -71489 & 2967 \\
\hline $377-35 s$ & 89 & 5 & 31 & $-67 r 94$ & 2910 \\
\hline $377-35 s$ & 89 & 6 & 30 & -67212 & 2987 \\
\hline $377-355$ & 89 & 7 & 39 & -67156 & 3032 \\
\hline $377-35 s$ & 89 & 8 & 31 & -67438 & 2998 \\
\hline
\end{tabular}


III. MAVAL PETROLEL RESERVE NO. 1 WATER INJECTION SUMMARIES 1989

\begin{tabular}{|c|c|c|c|c|c|}
\hline HELLL/SECT & YR & MONTH & $\begin{array}{l}\text { DAYS ON } \\
\text { INJECTION }\end{array}$ & $\begin{array}{l}\text { WATER } \\
\text { INJECTION }\end{array}$ & $\begin{array}{l}\text { TUBING } \\
\text { PRESSURE }\end{array}$ \\
\hline $377-35 s$ & 89 & 9 & 26 & .56170 & 3038 \\
\hline $377-35 s$ & 89 & 10 & 25 & -538887 & 2992 \\
\hline $377-35 s$ & 89 & 11 & 30 & -39316 & 2782 \\
\hline $377-35 s$ & 89 & 12 & 31 & -62814 & 2883 \\
\hline $388 x-355$ & 89 & 1 & 31 & -69423 & 3003 \\
\hline $388 x-355$ & 89 & 2 & 28 & -61146 & 3004 \\
\hline $388 x-35 s$ & 89 & 3 & 31 & -63423 & 3010 \\
\hline $388 x-35 s$ & 89 & 4 & 30 & -55989 & 3023 \\
\hline 398x-355 & 89 & 5 & 31 & -544485 & s̄ōî \\
\hline $388 x-355$ & 89 & 6 & 30 & -46242 & 2992 \\
\hline $388 x-355$ & 89 & 7 & 31 & -46912 & 2979 \\
\hline $368 x-355$ & 89 & 8 & 31 & -48715 & 3003 \\
\hline $388 x-355$ & 89 & 9 & 26 & -40535 & 2993 \\
\hline $388 x-35 s$ & 89 & 10 & 2.5 & -35612 & 2925 \\
\hline $388 x-35 s$ & 89 & 11 & 19 & -32658 & 2821 \\
\hline $388 x-35 s$ & 89 & 12 & 30 & -506666 & 2910 \\
\hline $316 x-365$ & 89 & 1 & 31 & -54689 & 3006 \\
\hline $316 x-365$ & 89 & 2 & 28 & -46894 & 3000 \\
\hline $316 x-365$ & 89 & 3 & 31 & -49695 & 3006 \\
\hline $316 x-365$ & 89 & 4 & 30 & -45141 & 3023 \\
\hline $316 x-365$ & 89 & 5 & 31 & -44533 & 3010 \\
\hline $316 x-365$ & 89 & 6 & 30 & -39487 & 3025 \\
\hline $316 x-365$ & 89 & 7 & 31 & -40112 & 3032 \\
\hline $316 x-365$ & 89 & 8 & 31 & -39532 & 3015 \\
\hline $316 x-365$ & 89 & 9 & 26 & -31626 & 3015 \\
\hline $316 x-365$ & 89 & 10 & 25 & -28097 & 2952 \\
\hline $316 x-365$ & 89 & 11 & 30 & -23681 & 2.829 \\
\hline $316 x-36 s$ & 89 & 12 & 30 & -37372 & 2950 \\
\hline $327 x-932$ & 89 & 9 & 31 & -100612 & 2089 \\
\hline $327 x-132$ & 89 & 2 & 28 & -85473 & 2107 \\
\hline $327 x-132$ & 89 & 3 & 31 & -92689 & 2084 \\
\hline $327 x-132$ & 89 & 4 & 30 & -95155 & 2168 \\
\hline $327 x-132$ & 89 & 5 & 31 & -40920 & 2053 \\
\hline $327 x-932$ & 89 & 6 & 30 & -89070 & 2053 \\
\hline $327 x-132$ & 89 & 7 & 31 & -90734 & 2130 \\
\hline $327 x-132$ & 89 & 8 & 31 & -83685 & 2111 \\
\hline $327 x-132$ & 89 & 9 & 30 & $-64,216$ & 1755 \\
\hline $327 x-132$ & 89 & 10 & 31 & -82273 & 1966 \\
\hline $327 x-132$ & 89 & 11 & 30 & -63834 & 2081 \\
\hline $327 x-132$ & $B 9$ & 12 & 26 & -58913 & 2164 \\
\hline $347 A-132$ & 89 & 1 & 31 & -100607 & 1597 \\
\hline $347 A-132$ & 89 & 2 & 28 & -89673 & 1811 \\
\hline $347 A-132$ & 89 & 3 & 31 & -96106 & 1806 \\
\hline $347 A-132$ & 89 & 4 & 30 & -73716 & 2057 \\
\hline $347 A-132$ & 89 & 5 & 31 & -73555 & 2062 \\
\hline $347 A-132$ & 89 & 6 & 30 & -79511 & 937 \\
\hline
\end{tabular}


II1. MAVAL PETROLEUM RESERVE MO. 1 WATER INJECTION SLMMARIES 1989

\begin{tabular}{|c|c|c|c|c|c|}
\hline WELL/SECT & YR & MONTH & $\begin{array}{l}\text { DAYS ON } \\
\text { INJECTION }\end{array}$ & $\begin{array}{l}\text { WATER } \\
\text { IHJECTION }\end{array}$ & $\begin{array}{l}\text { TUBIMG } \\
\text { PRESSURE }\end{array}$ \\
\hline $347 A-132$ & 89 & 7 & 31 & -90209 & 971 \\
\hline $347 A-132$ & 89 & 8 & 31 & .90577 & 1366 \\
\hline $347 A-132$ & 89 & 9 & 30 & -45002 & 2060 \\
\hline $347 A-132$ & 89 & 10 & 31 & -37211 & 2090 \\
\hline $347 A-132$ & 89 & 11 & 30 & -41699 & 2140 \\
\hline $347 A-132$ & 89 & 12 & 31 & -45736 & 2165 \\
\hline $367-132$ & 89 & 1 & 39 & -105057 & 998 \\
\hline $367-132$ & 89 & 2 & 28 & -91312 & 1018 \\
\hline $367-13 \overline{2}$ & 6ิ & 5 & 31 & $-9 \times 9879$ & iÛT4 \\
\hline $367-132$ & 89 & 4 & 30 & -90993 & 1057 \\
\hline $367-132$ & 89 & 5 & 31 & -102169 & 1135 \\
\hline $367-132$ & 89 & 6 & 30 & -118474 & 1307 \\
\hline $367-132$ & 89 & 7 & 31 & $\cdot 120883$ & 1482 \\
\hline $367-132$ & 89 & 8 & 31 & -121150 & 1577 \\
\hline $367-132$ & 89 & 9 & 30 & -140180 & 1810 \\
\hline $367-132$ & 89 & 10 & 31 & -136147 & 1880 \\
\hline $367-132$ & 89 & 11 & 30 & -141152 & 1872 \\
\hline $367-132$ & 89 & 12 & 31 & -120476 & 1511 \\
\hline $386-132$ & 89 & 1 & 31 & -105756 & 1795 \\
\hline $386-132$ & 89 & 2 & 28 & -92847 & 1800 \\
\hline $386-132$ & 89 & 3 & 31 & -92648 & 1600 \\
\hline $386-132$ & 89 & 4 & 30 & -101400 & 1677 \\
\hline $386-132$ & 89 & 5 & 31 & -98484 & 1760 \\
\hline $386-132$ & 89 & 6 & 30 & -100564 & 2072 \\
\hline $386-132$ & 89 & 7 & 31 & -101823 & 2113 \\
\hline $386-132$ & 89 & 8 & 31 & -98920 & 2100 \\
\hline $386-132$ & 89 & 9 & 30 & -111113 & 2042 \\
\hline $386-132$ & 89 & 10 & 31 & -105509 & 2156 \\
\hline $386-132$ & 89 & 11 & 30 & -98997 & 1933 \\
\hline $386-132$ & 89 & 12. & 31 & -123257 & 2200 \\
\hline $315-242$ & 89 & 1 & 29 & -103486 & 2129 \\
\hline $315-242$ & 89 & 2 & 2 & -7620 & 2100 \\
\hline $315-242$ & 89 & 3 & 0 & 0 & 2100 \\
\hline $315-242$ & 89 & 4 & 0 & 0 & 2100 \\
\hline $315-242$ & 89 & 5 & 30 & -100734 & 2187 \\
\hline $315-242$ & 89 & 6 & 30 & -94397 & 2270 \\
\hline $315-242$ & 89 & 7 & 31 & -93941 & 2311 \\
\hline $315-242$ & 89 & 8 & 29 & -89655 & 2302 \\
\hline $315-242$ & 89 & 9 & 30 & -79188 & 2117 \\
\hline $315-242$ & 89 & 10 & 31 & -91961 & 2173 \\
\hline $315-242$ & 89 & 11 & 30 & -82291 & 2179 \\
\hline $315-242$ & 89 & 12 & 31 & .95871 & 2265 \\
\hline $317-242$ & 89 & 1 & 31 & -35024 & 1997 \\
\hline $317-242$ & 89 & 2 & 28 & -336.44 & 2000 \\
\hline $317-242$ & 89 & 3 & 31 & -31064 & 2000 \\
\hline $317-247$ & 89 & 4 & 30 & -30360 & 2045 \\
\hline
\end{tabular}


III. MAYAL PETROLEUM RESERVE MO. 1 MATER INJECTION SUMMRIES 1989

\begin{tabular}{|c|c|c|c|c|c|}
\hline WELL/SECT & YR & MONTH & $\begin{array}{l}\text { DAYS ON } \\
\text { IHJECTION }\end{array}$ & $\begin{array}{l}\text { WATER } \\
\text { INJECTION }\end{array}$ & $\begin{array}{c}\text { TUBING } \\
\text { PRESSURE }\end{array}$ \\
\hline $317-242$ & 89 & 5 & 31 & -29295 & 2023 \\
\hline $317-242$ & 89 & 6 & 30 & -23414 & 2010 \\
\hline $317-242$ & 89 & 7 & 31 & -18448 & 2010 \\
\hline $317-242$ & 89 & 8 & 31 & -16457 & 2000 \\
\hline $317-242$ & 89 & 9 & 29 & -19571 & 2000 \\
\hline $317-242$ & 89 & 10 & 31 & -25422 & 2018 \\
\hline $317-242$ & 89 & 11 & 30 & -18582 & 2002 \\
\hline $317-242$ & 89 & 12 & 39 & -20296 & 1998 \\
\hline $32<-2<2$ & 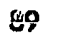 & $i$ & 31 & -120348 & 2235 \\
\hline $326-242$ & 89 & 2 & 28 & -106139 & 2279 \\
\hline $326-242$ & 89 & 3 & 31 & -128648 & 2310 \\
\hline $326-242$ & 89 & 4 & 30 & -120590 & 2327 \\
\hline $326-242$ & 89 & 5 & 31 & -105224 & 2153 \\
\hline $326-242$ & 89 & 6 & 30 & -107926 & 2165 \\
\hline $32.6-242$ & 89 & 7 & 31 & -110329 & 2019 \\
\hline $326-242$ & 89 & 8 & 31 & -126006 & 2305 \\
\hline $326-242$ & 89 & 9 & 30 & -98808 & 1958 \\
\hline $326-242$ & 89 & 10 & 31 & -97949 & 1687 \\
\hline 326.242 & 89 & 11 & 30 & -100108 & 1930 \\
\hline $326-242$ & 89 & 12 & 31 & -116254 & 2156 \\
\hline $337-242$ & 89 & 1 & 31 & -158679 & 1100 \\
\hline $337-242$ & 89 & 2 & 28 & -136364 & 1175 \\
\hline $337-242$ & 89 & 3 & 39 & -152476 & 1342 \\
\hline $337-242$ & 89 & 4 & 30 & -936672 & 1295 \\
\hline $337-242$ & 89 & 5 & 31 & -130320 & 1163 \\
\hline $337-242$ & 89 & 6 & 30 & -107902 & 902 \\
\hline $337-242$ & 89 & 7 & 29 & -99456 & 815 \\
\hline $337-242$ & 89 & 8 & 31 & -145660 & 1179 \\
\hline $337-242$ & 89 & 9 & 30 & -124933 & 1255 \\
\hline $337-242$ & 89 & 10 & 31 & - 118015 & 889 \\
\hline $337-242$ & 89 & 11 & 30 & -110463 & 929 \\
\hline $337-242$ & 89 & 12 & 31 & -117374 & 1300 \\
\hline $337 A-24 Z$ & 89 & 1 & 30 & -136519 & 1071 \\
\hline $337 A-242$ & 89 & 2 & 28 & -121399 & 1139 \\
\hline $337 A-242$ & 89 & 3 & 31 & -136496 & 1258 \\
\hline $337 A-242$ & 89 & 4 & 30 & -122662 & 1262 \\
\hline $337 A-24 z$ & 89 & 5 & 31 & -146158 & 1439 \\
\hline $337 A-24 Z$ & 89 & 6 & 30 & -143418 & 1370 \\
\hline $337 A-242$ & 89 & 7 & $3 i$ & -153797 & 1503 \\
\hline $337 A-242$ & 89 & 8 & 31 & -135533 & 1639 \\
\hline $337 A-242$ & 89 & 9 & 30 & -129726 & 1657 \\
\hline $337 A-262$ & 89 & 10 & 31 & -114040 & 1131 \\
\hline $337 A-242$ & 89 & 11 & 30 & -108851 & 1315 \\
\hline $337 A-262$ & 89 & 12 & 31 & -121578 & 1581 \\
\hline $338-242$ & 89 & 9 & 31 & -72426 & 1947 \\
\hline $338-242$ & 89 & 2 & 28 & -72948 & 1950 \\
\hline
\end{tabular}




\title{
APPENDIX H
}

\author{
SUMMARY OF NPR-1 1989 \\ DISPOSAL SITE MONITORING DATA \\ SUBMITTED TO THE \\ CALIFORNIA REGIONAL WATER QUALITY CONTROL BOARD \\ (ANNUAL REPORT)
}




\title{
Bechtel Petroleum Operations, Inc.
}

\author{
28590 Highway 119 \\ Túpman, California \\ Mail Address: P.O. Box 127. Tupman, CA 93276 \\ Tolephone: (805) 763-6000 \\ March 27, 1990
}

California Regional water

Quality Control Board

Central Valley Region

San Joaquin Watershed Branch of́rice

3614 East Ashlan Avenue

Fresno, CA 93276

Subject: 1989 ANNUAL WASTE DISCHARGE REPORT

RWQCB ORDER NO. 73-042 AND 73-141

Gentlemen:

Attached is the 1989 Annual Waste Discharge Report for on-site disposal at the $10 G$ and $27 R$ disposal sites located on Naval
Petroleum Reserve No. 1.

Yours truly,

BECHTEL PETROLEUM OPERATIONS, INC.

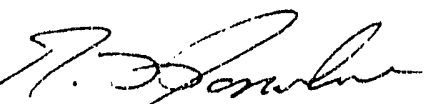

R. L. Donahoe

Manager, Environmental

Services

REC/MSM : rbe

CC: DNPRC

Operating Committee

Mr. Scott Sinith, CRWQCB

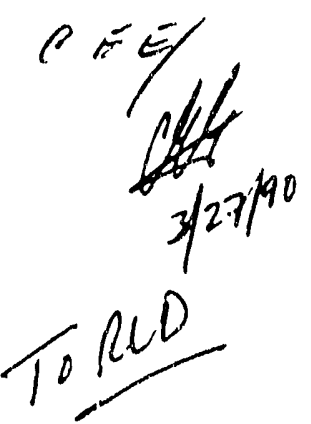




\section{R DISPOSAL SITE}

\begin{tabular}{|c|c|c|c|c|}
\hline MONTH & $\frac{\text { DRILLING MUD }}{(b b I s)}$ & $\frac{O I L / W A T E R}{(b b l s)}$ & $\frac{\text { OTHER }}{\text { (bbls) }}$ & TOTAL \\
\hline $\begin{array}{l}\text { January } \\
\text { February } \\
\text { March } \\
\text { April } \\
\text { May } \\
\text { June } \\
\text { July } \\
\text { August } \\
\text { September } \\
\text { October } \\
\text { November } \\
\text { December }\end{array}$ & $\begin{array}{c}140 \\
1,762 \\
0 \\
1.15 \\
545 \\
7,510 \\
17,100 \\
18,844 \\
25,583 \\
15,051 \\
8,550 \\
2,750\end{array}$ & $\begin{array}{l}17,477 \\
21,732 \\
25,190 \\
21,294 \\
23,300 \\
20,451 \\
11,910 \\
23,255 \\
12,129 \\
38,861 \\
34,695 \\
15,518\end{array}$ & $\begin{array}{c}0 \\
130^{2} \\
0 \\
35^{3} \\
480^{1} \\
1230^{1} \\
1330^{1} \\
275^{1} \\
845^{4} \\
487^{1} \\
1850^{1} \\
500^{1}\end{array}$ & $\begin{array}{l}17,617 \\
23,654 \\
25,190 \\
21,444 \\
24,325 \\
29,191 \\
30,340 \\
42,374 \\
38,557 \\
54,399 \\
45,095 \\
18,768\end{array}$ \\
\hline
\end{tabular}

\section{IOG DIBPOBAL SITE}

January
February
March
April
May
June
July
August
September
October
November
December

$\begin{aligned} & 6, 975 \\ & 12, 033 \\ & 7, 961 \\ & 2, 795 \\ & 320 \\ & 0 \\ & 0 \\ & 0 \\ & 0 \\ & 0 \\ & 0 \\ & 0\end{aligned}$

3,210
1,190
680
7,450
1,140
0
0
0
0
0
0
0

$\begin{array}{cc}0 & 10,185 \\ 260^{1} & 13,483 \\ 170^{1} & 9,811 \\ 960^{1} & 11,205 \\ 0 & 1,460 \\ 0 & 0 \\ 0 & 0 \\ 0 & 0 \\ 0 & 0 \\ 0 & 0 \\ 0 & 0 \\ 0 & 0\end{array}$

cement/water

$100 \mathrm{bbls}$ cement/water, 30 bbls polymer glycol

frac gel 


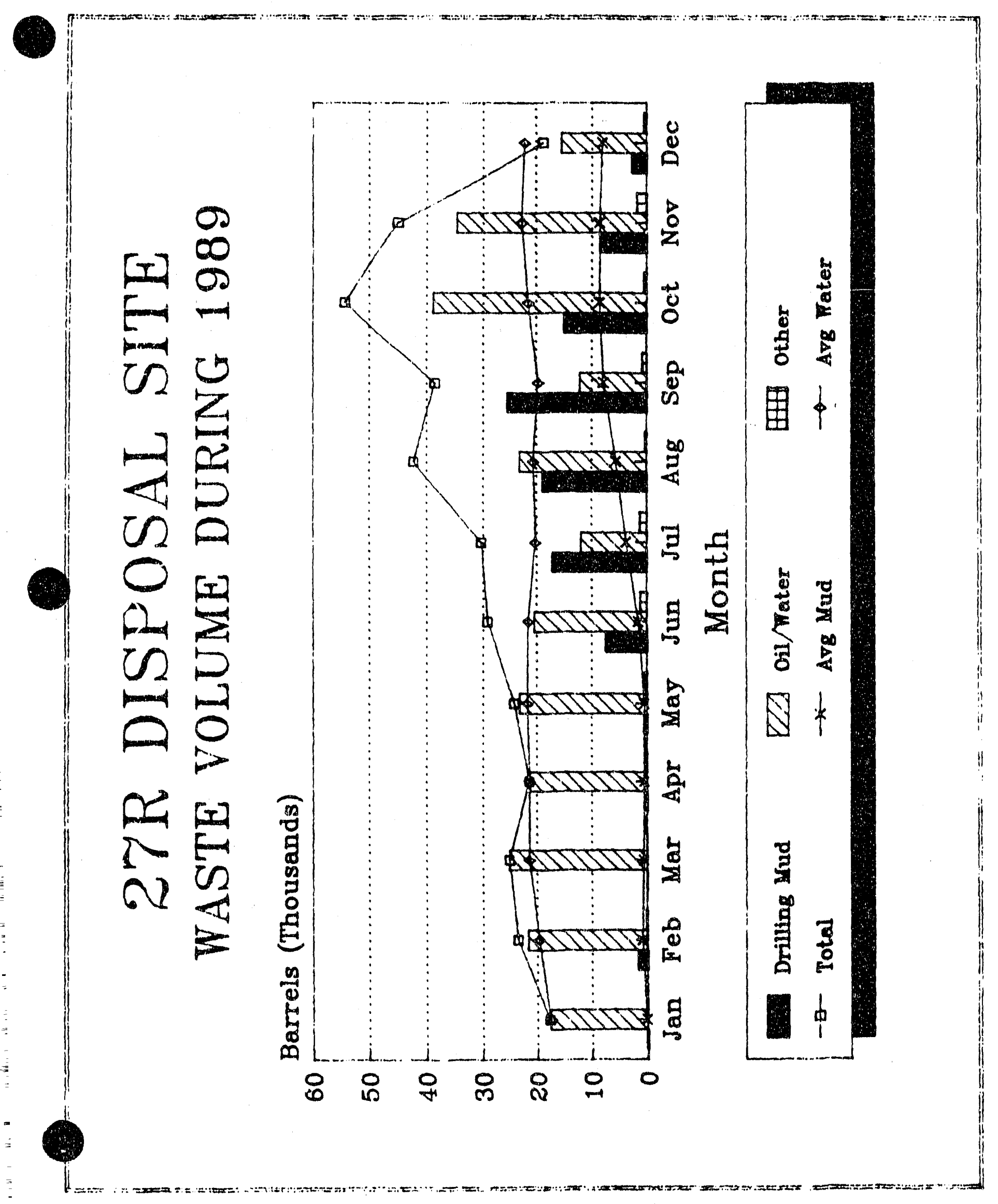




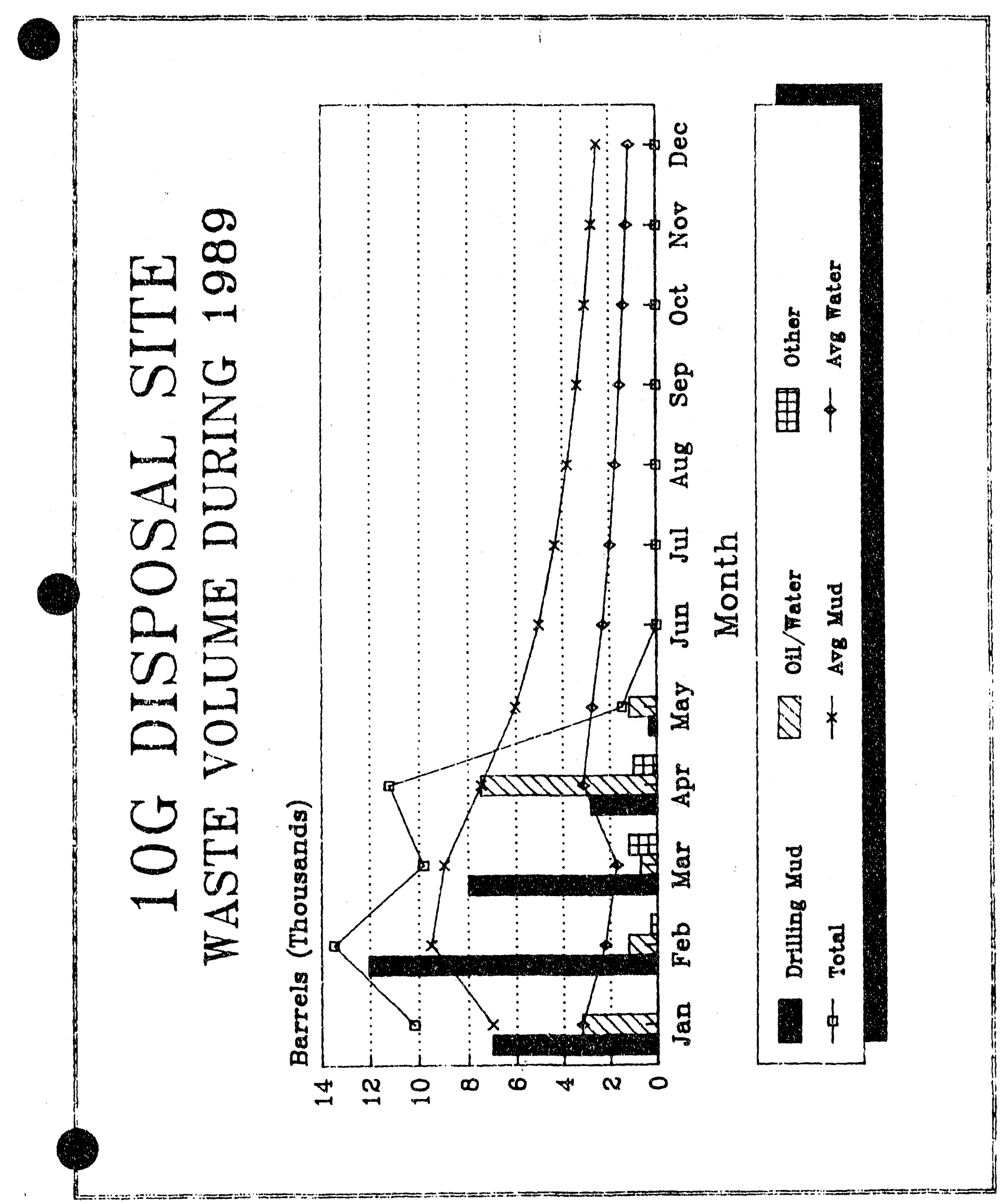




\section{APPENDIX I}

SUMMARY OF NPR-1 1989 SAMPLES TAKEN FOR WATER, WASTE AND PCBS 
1989 Semples raken

\begin{tabular}{|c|c|c|c|c|c|c|c|c|c|c|c|c|}
\hline de & $\begin{array}{c}\text { Control/ } \\
\text { File }\end{array}$ & Date & \multicolumn{2}{|l|}{ Semple } & Test & Test & Test & Test & Lab Used & Sampled By & SR \# & Status \\
\hline & I/ Wa & oil & & & & & & & & & & \\
\hline 1 & 89025 & $4 / 20$ & $10 G$ LACT & Crude oil & BTX & 8020 & Metals & & $\mathrm{BC}$ & RTA & ADH977 & Recr. \\
\hline 1 & 89026 & $4 / 20$ & $25 S$ LACT & Crude oil & BTX & 8020 & Metals & & $\mathrm{BC}$ & RTA & ADK977 & Recv. \\
\hline 1 & 89027 & $4 / 20$ & $242 \mathrm{LACT}$ & Crude oil & BrX & 8020 & Metals & & $\mathrm{BC}$ & RTA & ADHQT7 & Recv. \\
\hline 1 & 89028 & $4 / 20$ & $262 \mathrm{LACT}$ & Crude oil & BTX & 8020 & Metals & & $B C$ & RTA & ADH977 & Recv. \\
\hline 1 & 89030 & $4 / 27$ & $18 G$ LACT & Crude oil & 8020 & Metals & & & $\mathrm{BC}$ & RTA & ADH9T7 & Recv. \\
\hline 1 & 89033 & $5 / 12$ & 30R Comp & Crankcase oil & Lead & Arsen & Ced PCB & Chlor & $B C$ & RTA & $A D 1874$ & Recv. \\
\hline 1 & 89044 & $7 / 13$ & $25 S$ LACT & Crude & BTX & 8020 & Metals & & $B C$ & MSM & ADL 104 & Recv. \\
\hline 1 & 89047 & $7 / 13$ & $10 G$ LACT & Crude & BTX & 8020 & Metals & & $\mathrm{BC}$ & MSM & ADL 104 & Recv. \\
\hline 1 & 89049 & $7 / 13$ & $18 G$ LACT & Crude & BTX & 8020 & Metals & & $\mathrm{BC}$ & MSM & ADL 104 & Recr. \\
\hline 1 & 89050 & $7 / 13$ & 242 LACT & Crude & BTX & 8020 & Metals & & $\mathrm{BC}$ & MSM & ADL 104 & Recr. \\
\hline 1 & 89052 & $7 / 13$ & $262 \mathrm{LACT}$ & Crude & BTX & 8020 & Metals & & $\mathrm{BC}$ & MSM & ADL 104 & Recv. \\
\hline 1 & 89084 & $9 / 12$ & $357-8 R$ & Crankcase oil & Metals & & & & $\mathrm{BC}$ & Wayne $H$. & ADO 877 & Recr. \\
\hline 1 & 89106 & $11 / 17$ & $30 R \quad K-71$ & Crankcase oil & Lead & Arsen & Cad PCB & Chlor & $B C$ & Richard H. & ADN 924 & Recv. \\
\hline 1 & 89107 & $11 / 17$ & $30 R K-70$ & Crankcase oil & Lead & Arsen & Cad PCB & Chlor & $\mathrm{BC}$ & Richard $H$. & ADN 924 & Recv. \\
\hline 1 & 89112 & $12 / 8$ & LTS 2 & Xylene & 8020 & & & & $\mathrm{BC}$ & MSM & ADP 586 & Recr. \\
\hline
\end{tabular}

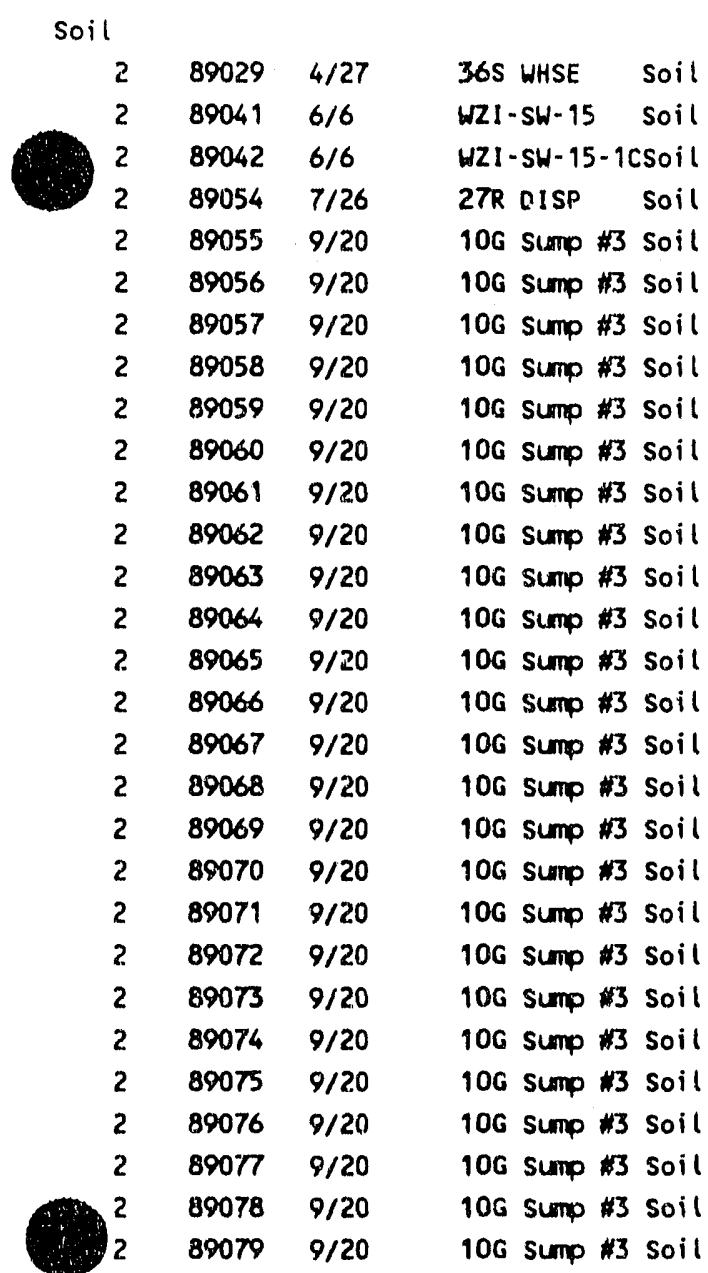

\begin{tabular}{|c|c|c|c|c|c|}
\hline 8010 & 8020 & Metals & PCBS & $B C$ & RTA \\
\hline \multicolumn{4}{|c|}{ BTX-TPH } & $B C$ & RTA \\
\hline \multicolumn{4}{|c|}{ BTX-TPH } & $B C$ & RTA \\
\hline 8020 & 8010 & Metals & Fishtox & $B C$ & MSM \\
\hline 3050 & 7061 & & & $B C$ & $\mathrm{BDH}$ \\
\hline 3050 & 7061 & & & $B C$ & $\mathrm{BOH}$ \\
\hline 3050 & 7061 & & & BC & BDH \\
\hline 3050 & 7061 & & & $B C$ & BDH \\
\hline 3050 & 7061 & & & $B C$ & BDH \\
\hline 3050 & 7061 & & & $B C$ & $\mathrm{BDH}$ \\
\hline 3050 & 7061 & & & $\mathrm{BC}$ & BDH \\
\hline 3050 & 7061 & & & $B C$ & $\mathrm{BDH}$ \\
\hline 3050 & 7061 & & & BC & BDH \\
\hline 3050 & 7061 & & & $B C$ & $\mathrm{BDH}$ \\
\hline 3050 & 7061 & & & BC & BDH \\
\hline 3050 & 7061 & & & BC & BDH \\
\hline 3050 & 7061 & & & BC & BDH \\
\hline 3050 & 7061 & & & $\mathrm{BC}$ & $\mathrm{BDH}$ \\
\hline 3050 & 7061 & & & $B C$ & BDH \\
\hline 3050 & 7061 & & & $B C$ & BOH \\
\hline 3050 & 7061 & & & $B C$ & BDH \\
\hline 3050 & 7061 & & & BC & $B D H$ \\
\hline 3050 & 7061 & & & $B C$ & BDH \\
\hline 3050 & 7061 & & & $B C$ & BDH \\
\hline 3050 & 7061 & & & $B C$ & BDH \\
\hline 3050 & 7061 & & & $\mathrm{BC}$ & $\mathrm{BDH}$ \\
\hline 3050 & 7061 & & & $B C$ & $\mathrm{BDH}$ \\
\hline 3050 & 7061 & & & $B C$ & $\mathrm{BDH}$ \\
\hline 3050 & 7061 & & & BC & $B D K$ \\
\hline
\end{tabular}

\begin{tabular}{|c|c|c|}
\hline & & \\
\hline$\pi 8$ & 930 & $c V$ \\
\hline & & $c V$ \\
\hline & 526 & RecV \\
\hline & 903 & $n$ \\
\hline & & P \\
\hline & 9 & P \\
\hline & 903 & P \\
\hline & 903 & $p$ \\
\hline & 903 & P \\
\hline & 903 & P \\
\hline & & 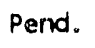 \\
\hline & 19 & nd \\
\hline & & $p$ \\
\hline & 90 & \\
\hline & & Pend \\
\hline & & \\
\hline & & $\mathrm{P}$ \\
\hline & & nd \\
\hline & 19 & \\
\hline & & Pend \\
\hline & & Pen \\
\hline & & \\
\hline M & & \\
\hline & 1 & Pend \\
\hline & & 0 \\
\hline & & \\
\hline & & \\
\hline & & \\
\hline
\end{tabular}




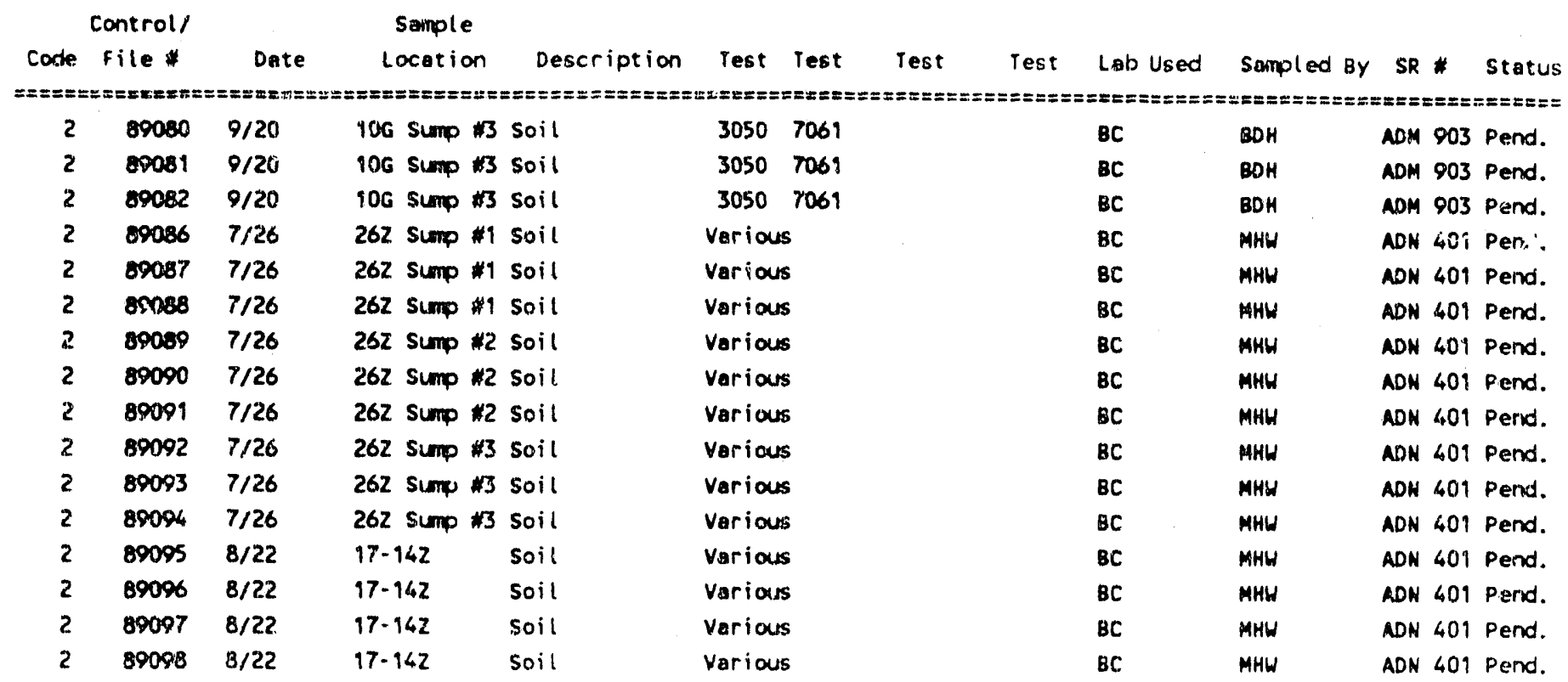

Tank Rot tons

$\begin{array}{rrr}3 & 89001 & 1 / 26 \\ 3 & 89039 & 6 / 7 \\ 3 & 89040 & 6 / 7 \\ 3 & 89043 & 6 / 27\end{array}$

Acid

$48908310 / 8$

Waste yater

$\begin{array}{lllll}6 & 89021 & 4 / 20 & 10 G \text { LACT } & W / W \\ 6 & 89022 & 4 / 20 & 25 S \text { LACT } & W / W \\ 6 & 89023 & 4 / 20 & 242 \text { LACT } & W / W \\ 6 & 89024 & 4 / 20 & 262 \text { LACT } & W / W \\ 6 & 89031 & 4 / 27 & 18 G \text { LACT } & W / W \\ 6 & 89032 & 4 / 27 & 27 R \text { WAST } & W / W \\ 6 & 89045 & 7 / 13 & 25 S \text { LACT } & W / W \\ 6 & 89040 & 7 / 13 & 10 G \text { LACT } & W / W \\ 6 & 89048 & 7 / 13 & 1 B G \text { LACT } & W / W \\ 6 & 89051 & 7 / 13 & 262 \text { LACT } & W / W \\ 6 & 89053 & 7 / 13 & 262 \text { LACT } & W / W\end{array}$

Transformers

7

$7 \quad 09005$

$09002 \quad 315$

09004212

Uwx 18734

UnN 17648

UwX 10504

unx 17626

$89005 \quad 2 / 10$

B9006 $2 / 10$

LenX 17651

$89007 \quad 2 / 10$

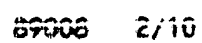

$\begin{array}{lll}\text { Uux } 18012 & \text { Tronsf. } & \text { PCBs } \\ \text { UNx inols } & \text { Transf. } & \text { PCBS }\end{array}$
Run TK bottom STLC Barium Metals Flamm. Zalco RTA

$W / W$

$H / W$

TK Btms

TK Btm sludge 8010,8070 , Metals, BC

TK Btm sludge 8010,8070 , Metals $B C$

80108020 Hetals Fishtox BC
PH Metals

$B C$

MSM

ADO 418 ReCV.

TDS chlor EPA 602
TDS chlor EPA 602
TDS chlor EPA 602
TDS chlor EPA 602
TOS chlor EPA 602
EPA 502.2 Hetals
TOS chlor EPA 503.1
TDS chlor EPA 503.1
TDS chlor EPA 503.1
TOS chlor EPA 503.1
TDS chlor EPA 503.1

PCBS

PCBs

PCBS

PCBS

PCBS

PCBS

$\begin{array}{ll}\text { BC } & \text { RTA } \\ \text { BC } & \text { RTA } \\ \text { BC } & \text { RTA } \\ \text { BC } & \text { RTA } \\ \text { BC } & \text { RTA } \\ \text { BC } & \text { RTA } \\ \text { BC } & \text { MSM } \\ \text { BC } & \text { MSM } \\ \text { BC } & \text { MSM } \\ \text { BC } & \text { MSM } \\ \text { BC } & \text { MSM }\end{array}$

$\begin{array}{llll}\text { BC } & \text { RTA } & P 89-00469 & \text { RACV. } \\ \text { BC } & \text { RTA } & \text { P89-00469 } & \text { ReCV. } \\ \text { BC } & \text { RTA } & \text { P89.00469 } & \text { ReCV. } \\ \text { SHC } & \text { RTA } & \text { P89-00478 ReCV. } \\ \text { SHC } & \text { RTA } & P 89-00478 & \text { ReCV. } \\ \text { SMC } & \text { RTA } & \text { P89-00478 } & \text { ReCV. } \\ \text { SMC } & \text { RTA } & \text { P89-00478 ReCV. }\end{array}$




\begin{tabular}{|c|c|c|c|c|c|c|c|c|c|c|}
\hline \multicolumn{2}{|c|}{ Control/ } & \multicolumn{3}{|c|}{ Sample } & \multirow[b]{2}{*}{ Test Test } & \multirow{3}{*}{ 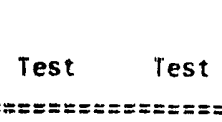 } & \multirow[b]{2}{*}{ Lab Used } & \multirow{2}{*}{\multicolumn{2}{|c|}{ Sampled BY SR \# }} & \multirow[b]{2}{*}{ Status } \\
\hline code & File & Date & Location & Description & & & & & & \\
\hline \multicolumn{10}{|c|}{ 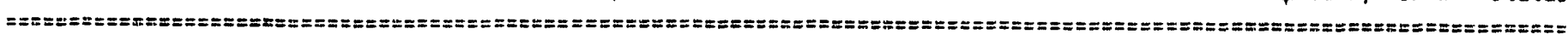 } & \\
\hline 7 & 89009 & $2 . / 10$ & UNK 18606 & Transf. & PCBs & & $\sin$ & RTA & P89-00478 & Recr. \\
\hline 7 & 89010 & $2 / 16$ & UwX 19178 & Iransf. & PCBS & & SMC & RTA & P89-00478 & Recr. \\
\hline 7 & 89011 & $2 / 16$ & UNX 19177 & Transf. & PCBs & & SHC & RTA & P89-00478 & Recv. \\
\hline 7 & 89012 & $2 / 16$ & UNX 19176 & Transf. & PCBs & & SMC & RTA & P89-00478 & Recv. \\
\hline 7 & 89013 & $2 / 16$ & UNX 19094 & Transf. & PCEs & & SNC & RTA & $P 89-00478$ & Recr. \\
\hline 7 & 89014 & $2 / 16$ & UNX 19095 & Transf. & PCBs & & SMC & RTA & P89-00478 & Recv. \\
\hline 7 & 89015 & $2 / 16$ & UNX 19096 & Transf. & PCBs & & SMC & RTA & $P 89-00478$ & Reer. \\
\hline 7 & 89016 & $4 / 13$ & UNX 14390 & Trans $\uparrow$. & PCBS & & $B C$ & RTA & ADH797 & Recr. \\
\hline 7 & 89017 & $4 / 43$ & UNK 14393 & Transf. & PCBS & & BC & RTA & ADH797 & Recv. \\
\hline 7 & 89018 & $4 / 13$ & UNX 16483 & Transf. & PCBS & & $\mathrm{BC}$ & RTA & АDH797 & Recr. \\
\hline 7 & 89019 & $4 / 13$ & UNX 16485 & Transf. & PCBs & & $B C$ & RTA & ADH797 & Recy. \\
\hline 7 & 89020 & $4 / 93$ & UNX 16484 & Transf. & PC8s & & $B C$ & RTA & ADH797 & Recr. \\
\hline 7 & 89034 & $5 / 31$ & UNX 50633 & Transf. & PCBS & & $B C$ & RTA & AOJ574 & Recr. \\
\hline 7 & 89035 & $5 / 31$ & UNX 50632 & Transt. & PCBs & & $\mathrm{BC}$ & RTA & $A D J 574$ & Recr. \\
\hline 7 & 89036 & $5 / 31$ & UNX 19125 & Transf. & PCBS & & $B C$ & RTA & ADJ574 & Recr. \\
\hline 7 & 89037 & $5 / 31$ & UNX 14820 & Trans $f$ & PCBS & & $B C$ & RTA & AD 5574 & Rerv. \\
\hline 7 & 89038 & $5 / 31$ & UNX 14809 & Transt. & PCBS & & $8 C$ & R.TA & AD 1574 & Recr. \\
\hline 7 & 89099 & $11 / 10$ & UNX 16967 & Trans. & PCE'S & & $B C$ & MSM & ADN 622 & Recv. \\
\hline 7 & 89100 & $11 / 10$ & UNX 16958 & Trans. & PCB'S & & $B C$ & MSM & ADN 622 & Recv. \\
\hline$\gamma$ & 89101 & $19 / 10$ & UNK 16969 & Trans. & PCB'S & & BC & MSM & NON 622 & Recv. \\
\hline 7 & 89102 & $11 / 10$ & UNA 17004 & Trans. & PCB'S & & $\mathrm{BC}$ & MSM & ADN 622 & Recr. \\
\hline 7 & 89103 & $11 / 10$ & UNX 17005 & Trans. & PCA'S & & $\mathrm{BC}$ & MSM & ADH 622 & Recr. \\
\hline 7 & 89104 & $11 / 10$ & UNX 17006 & Trans. & PCB'S & & $B C$ & MSM & ADN 622 & Recy. \\
\hline 7 & 89191 & $12 / 8$ & UNX 16969 & Trans. & PCB'S & & $B C$ & MSM & NOP 587 & Recr. \\
\hline \multicolumn{11}{|c|}{ Miscel laneous } \\
\hline 8 & 89085 & $10 / 18$ & $35 R$ & Soda ash & Sode ash & & $B C$ & MSM & ADO 876 & Recr. \\
\hline 8 & 89105 & $11 / 17$ & $2 B$ Yard & Vessel insul. & Asbestos & & $B C$ & MSM & ADN 892 & Recr. \\
\hline 8 & 89108 & $12 / 1$ & $36 s$ whse. & Chromium & $C R+6$ & & $B C$ & MSM & NOP 371 & Recv. \\
\hline 8 & 89109 & $12 / 9$ & 365 thse. & Chromiun & $C R+6$ & & $B C$ & MSM & ADP 371 & Recr. \\
\hline$B$ & 89110 & $12 / 1$ & 365 whse. & Chromiun & $C R+6$ & & BC & MSM & ADP 379 & Recr. \\
\hline
\end{tabular}




\section{APPENDIX J}

DESCRIPTION OF SITE GEOLOGY AND GROUNDWATER AQUIFERS 


\section{TABLE OF CONTENTS APPENDIX J}

Topography

Major Water Bodies

Groundwater

Kern County Water Agency Maps

Depth to Perched Groundwater

Groundwater Quality Map (Unconfined Aquifer)

Groundwater Quality Map (Confined Aquifer)

Depth to Groundwater

Groundwater Surface Elevation Map 


\section{TOPOGRAPHY}

NPR-1, which encompasses most of the Elk Hills, is characterized by a low elongated swell about 20 miles long and 7 miles wide that protrudes about 1,000 feet above the surrounding valley floor. Elevations within NPR-1 range from 290 feet above sea level on the valley floor at the northeastern boundary to 1,551 feet along the main ridge in the western part.

In general, the terrain of NPR-1 is characterized by numerous, rounded divides and smooth slopes. A large number of ephemeral/intermittent streams draining the hills have created a highly dissected stream pattern of gullies and channels. Sediments eroded from the higher elevations have been deposited along the base of the hills, resulting in a smooth topographic transition into the adjacent valley.

The topography of NPR-2, which includes portions of the Buena Vista and Midway Valleys and the Buena Vista Hills, is quite similar to that of NPR-1. Elevations within NPR-2 range from 400 feet above sea level in the Buena Vista Valley to 1,288 feet in the southeasterly section of the Buena Vista Hills.

\section{MAJOR WATER BODIES}

NPR-1 and NPR-2 are situated within the boundaries of the Tulare Lake Basin. Surface and groundwater flows within the basin converge toward the basin's central valley floor. In the past, this convergence resulted in the development of several large lakes (e.g., Tulare, Buena Vista, and Kern). However, upstream diversions, heavy groundwater pumping, and high evaporation rates have helped to reduce these lakes to dry lakebeds.

NPR-1 and 2 have relatively limited water resources. Less than 1 percent of the basin's

total surface flow originates from the NPRC with Buena Vista Creek, in Buena Vista Valley, carrying most of it. This flow occurs entirely in the form of intermittent streams 
that have sustained flows only during and immediately after periods of heavy precipitation. These surface flows seldom reach the valley floor; instead, they tend to rapidly percolate into their stream beds. Buena Vista Creek did flow into the Buena Vista lakebed, east of the NPRC until blocked by the California Aqueduct and dikes to protect the Aqueduct.

Although stream flow is intermittent, certain surface water features of NPRC have been designated to be waters of the United States by the Environmental Protection Agency. Sandy Creek on NPR-2 was declared a regulated waterway as was Buena Vista Creek and its tributaries and Broad Creek. The California Aqueduct, a major conduit of freshwater for Los Angeles and southern California, borders NPRC to the north, east, and south and is located within the NPR-1 boundaries in Sections 23S, 24S, and 25S, and within the NPR-2 boundaries in Sections 12D and 13D.

\section{GROUNDWATER}

Groundwater conditions on NPRC vary from east to west and from north to south. Potable groundwater - below $2000 \mathrm{ppm}$ total dissolved solids (TDS) - is found on the north and east sides of NPRC (See Kern County Water Agency maps following). Groundwater pumped from the Tulare zone on NPR-1 has a total dissolved solids concentration from 4000 to $6000 \mathrm{ppm}$ TDS (Sections 8R, 18G, 13B and 14B).

Two aquifers are found on most of NPRC with the lower aquifer confined. A clay layer exists on portions of NPRC within the lower Tulare and acts as the confining strata separating the two aquifers. On the eastern side of NPR-1, the clay separating aquifers doesn't exist, so the lower aquifer may not be confined except in areas where the clay may extend onto NPR-1 (See KCWA map attached).

The Corcoran clay divides the upper Tulare into confined and unconfined aquifers in the adjacent San Joaquin Valley. Often the water below the Corcoran clay in the San Joaquin 
Valley is of higher quality than the water above due to contamination from surface activities.

NPR-1 has a program underway to eliminate sumps and Tulare injection on NPR-1 while concurrently initiating a major produced water reuse program. This activity will reduce the potential for affecting off-site groundwater.

Sumps and other surface discharges are being eliminated on NPR-2, and are mainly being replaced by Tulare injection wells. Studies have been made of NPR-2 and adjacent areas under the direction of the Regional Water Quality Control Board, but the "Board" has not yet acted on the studies. 


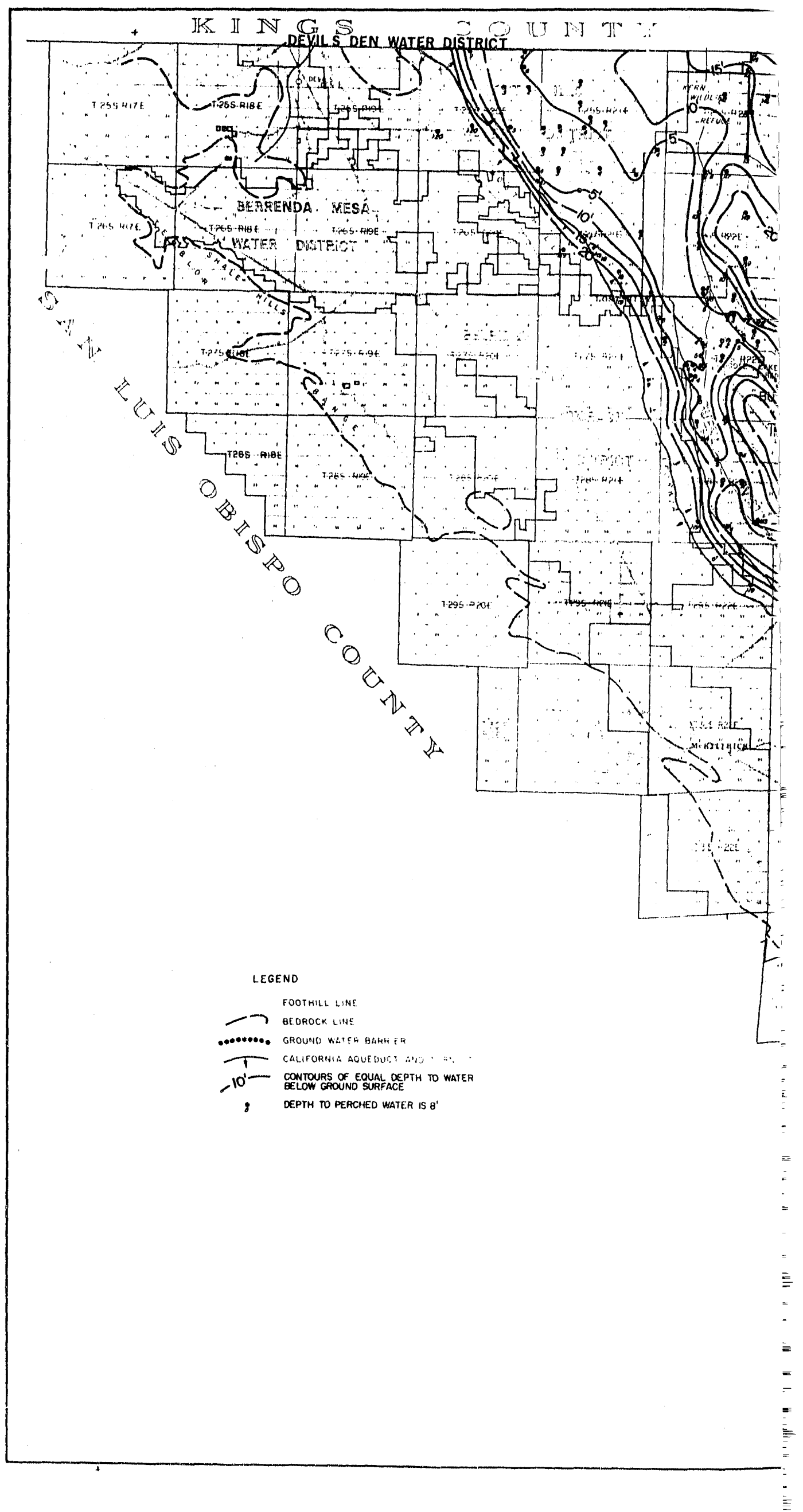




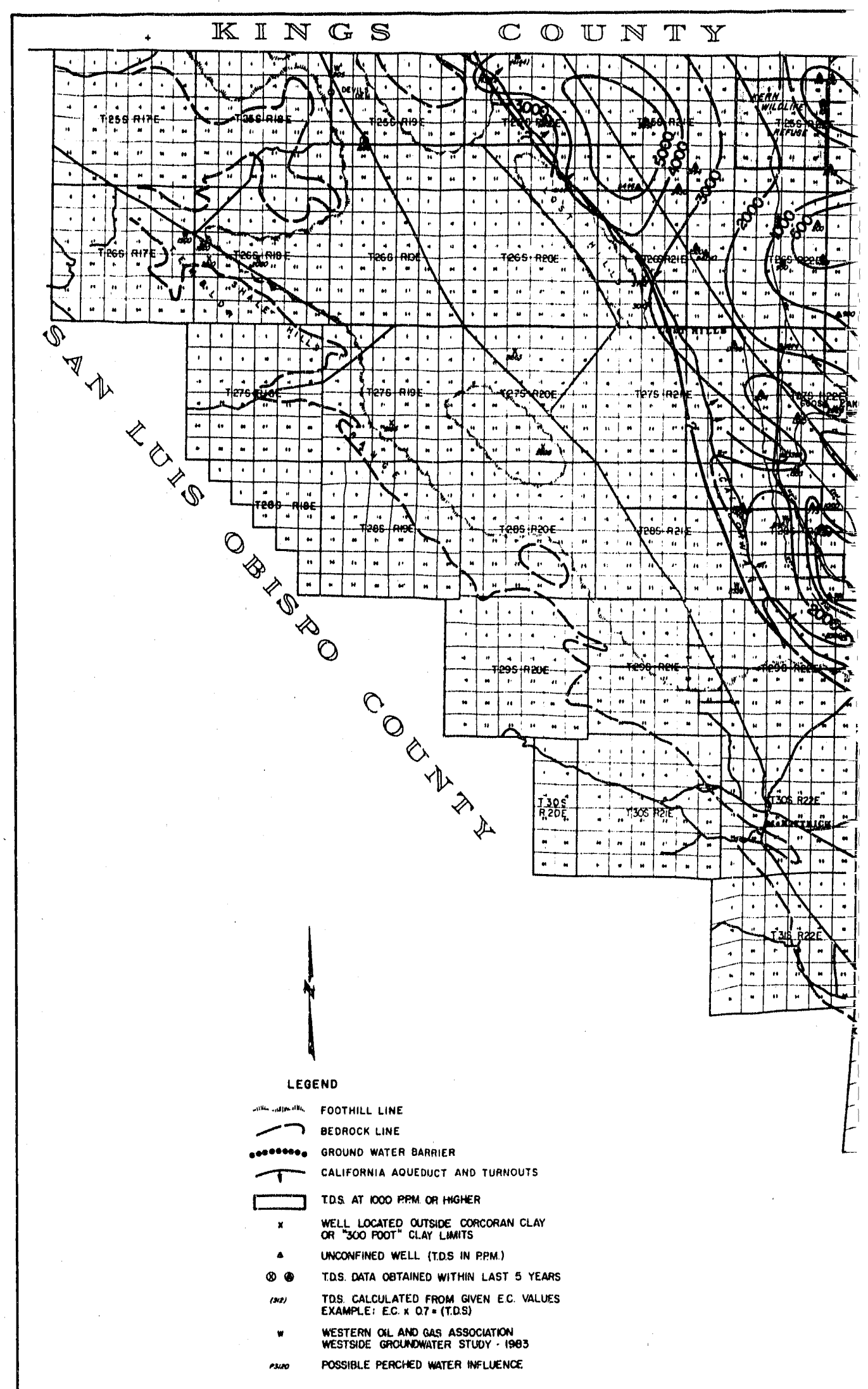


\begin{tabular}{llllll|lll}
$\mathbb{T}$ & $\mathbb{U}$ & $\mathbb{I}$ & $\mathbb{A}$ & $\mathbb{R}$ & $\mathbb{E}$ & $\mathbb{C}$ & $\mathbb{O}$
\end{tabular}

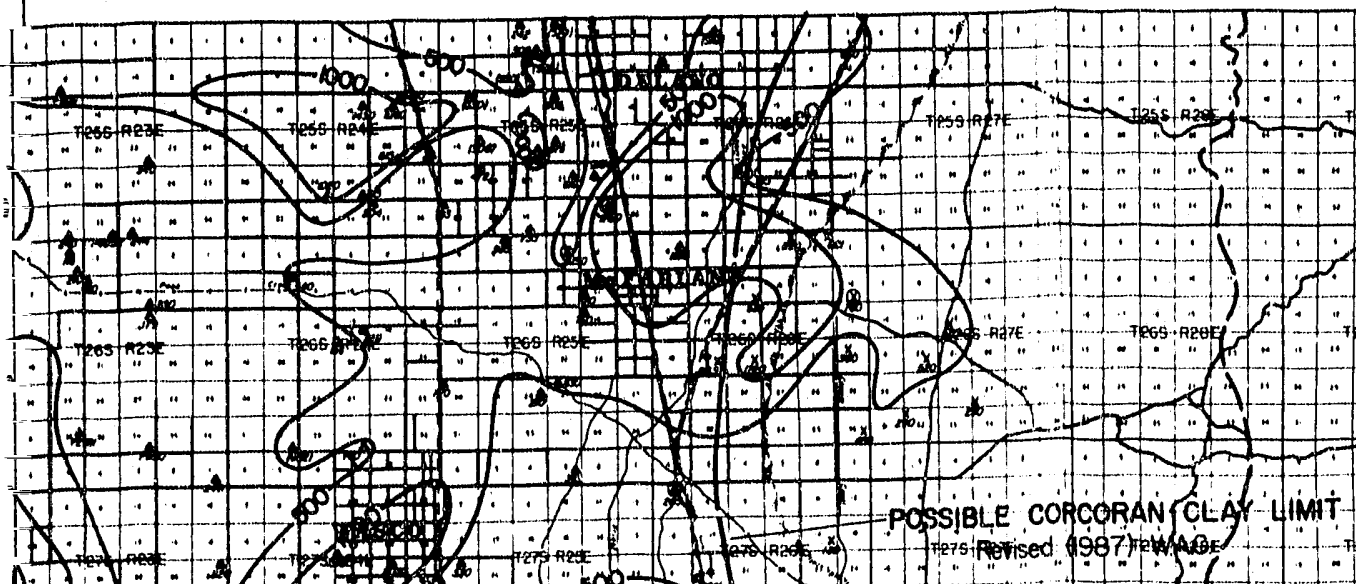

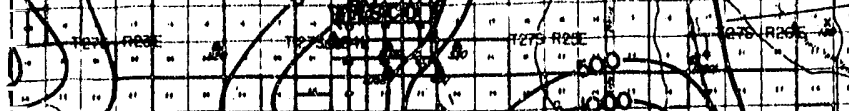

(1."

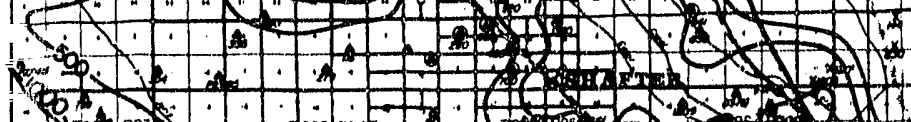

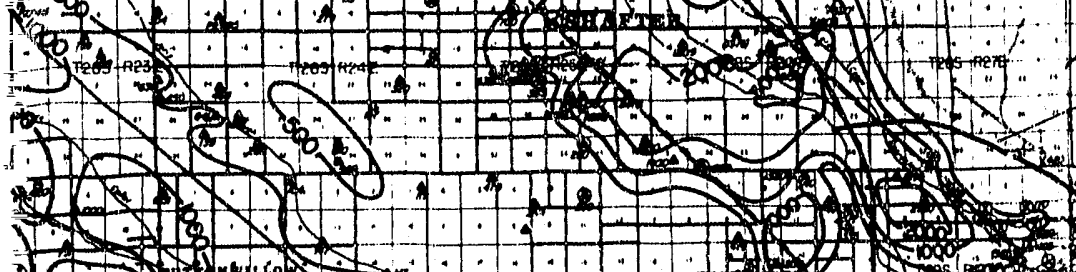

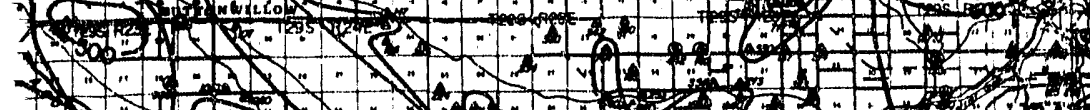

(1)

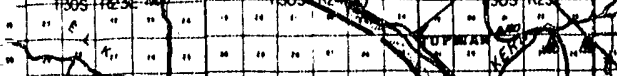

"17."

$=$

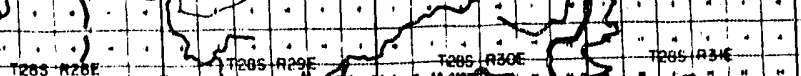

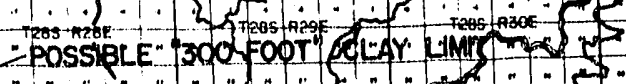

GROUNDWATER QUALITY MAP

UNCONFINED AQUIFER
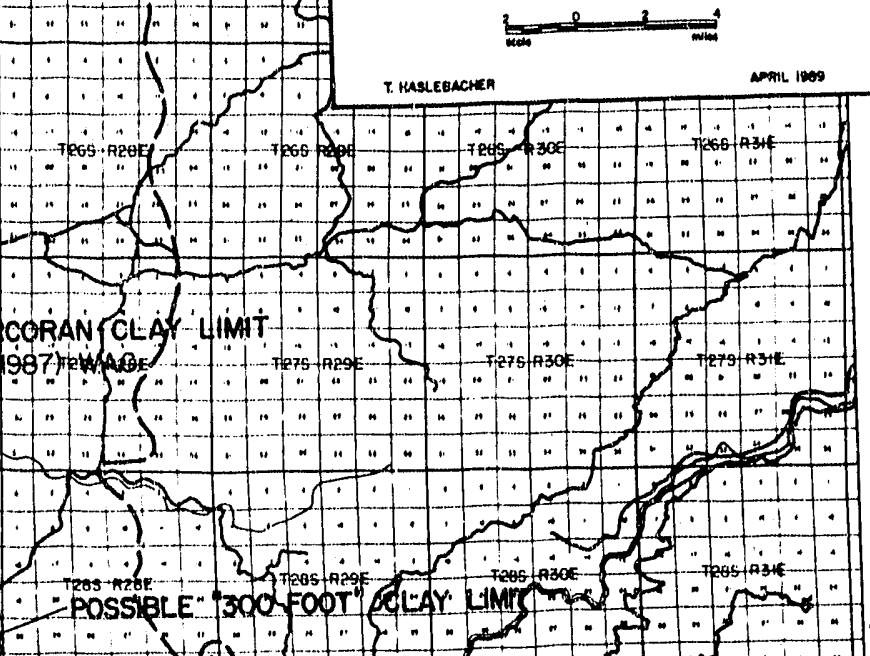

$\left(\log ^{\circ}\right.$

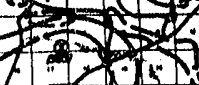

(3)


ए $\mathbb{E} \mathbb{I}$ "TV U IF $\mathbb{A}$ 


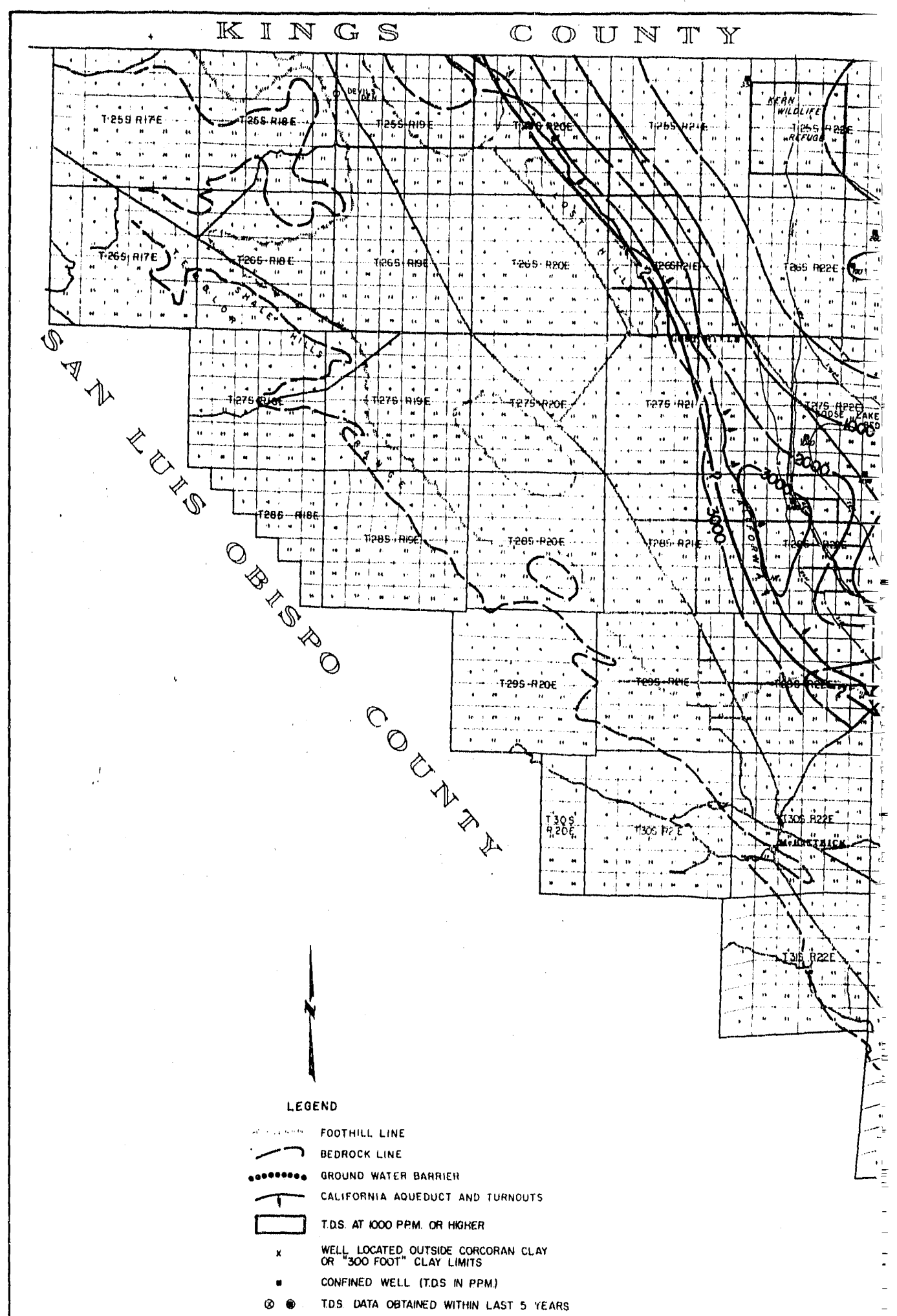




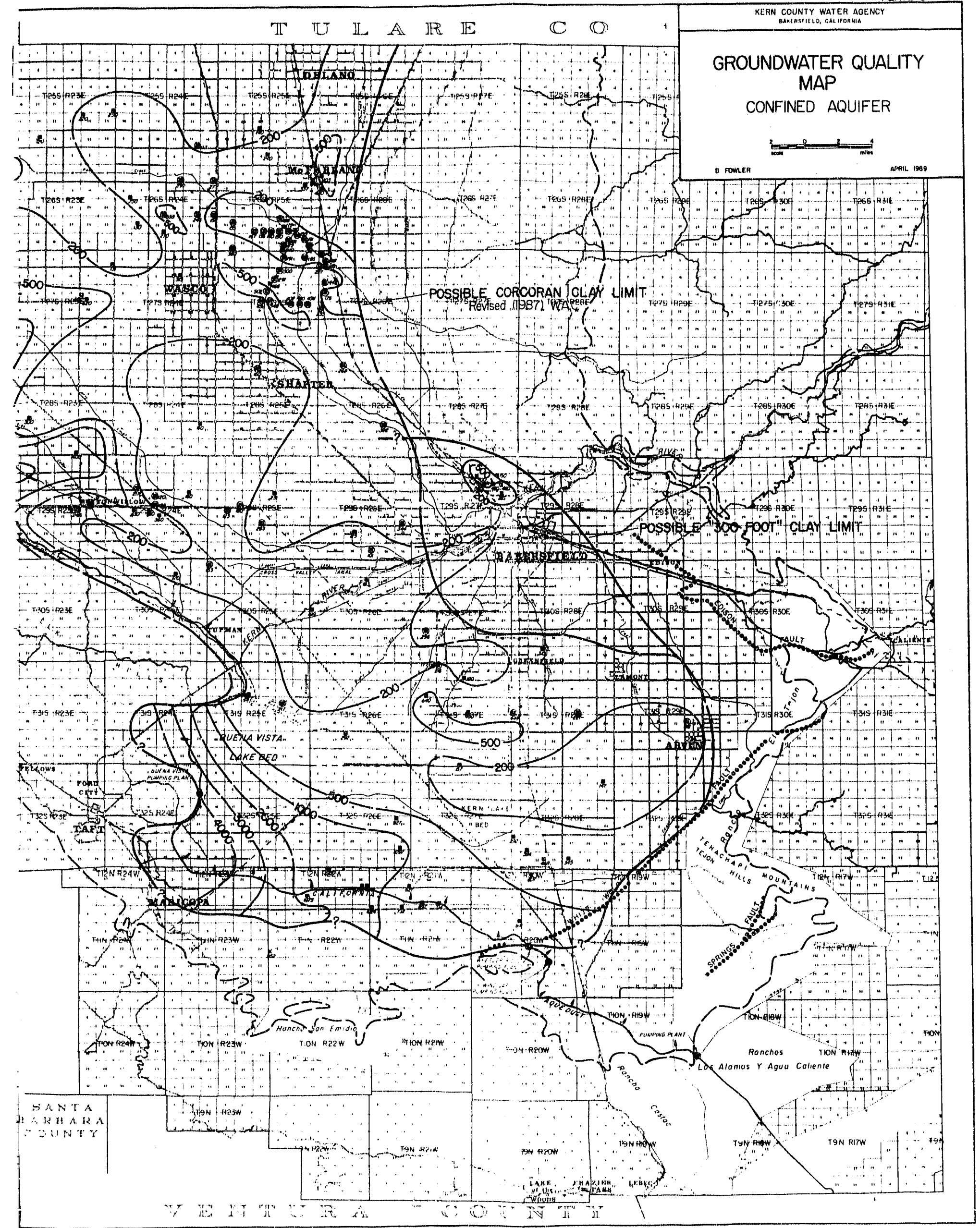




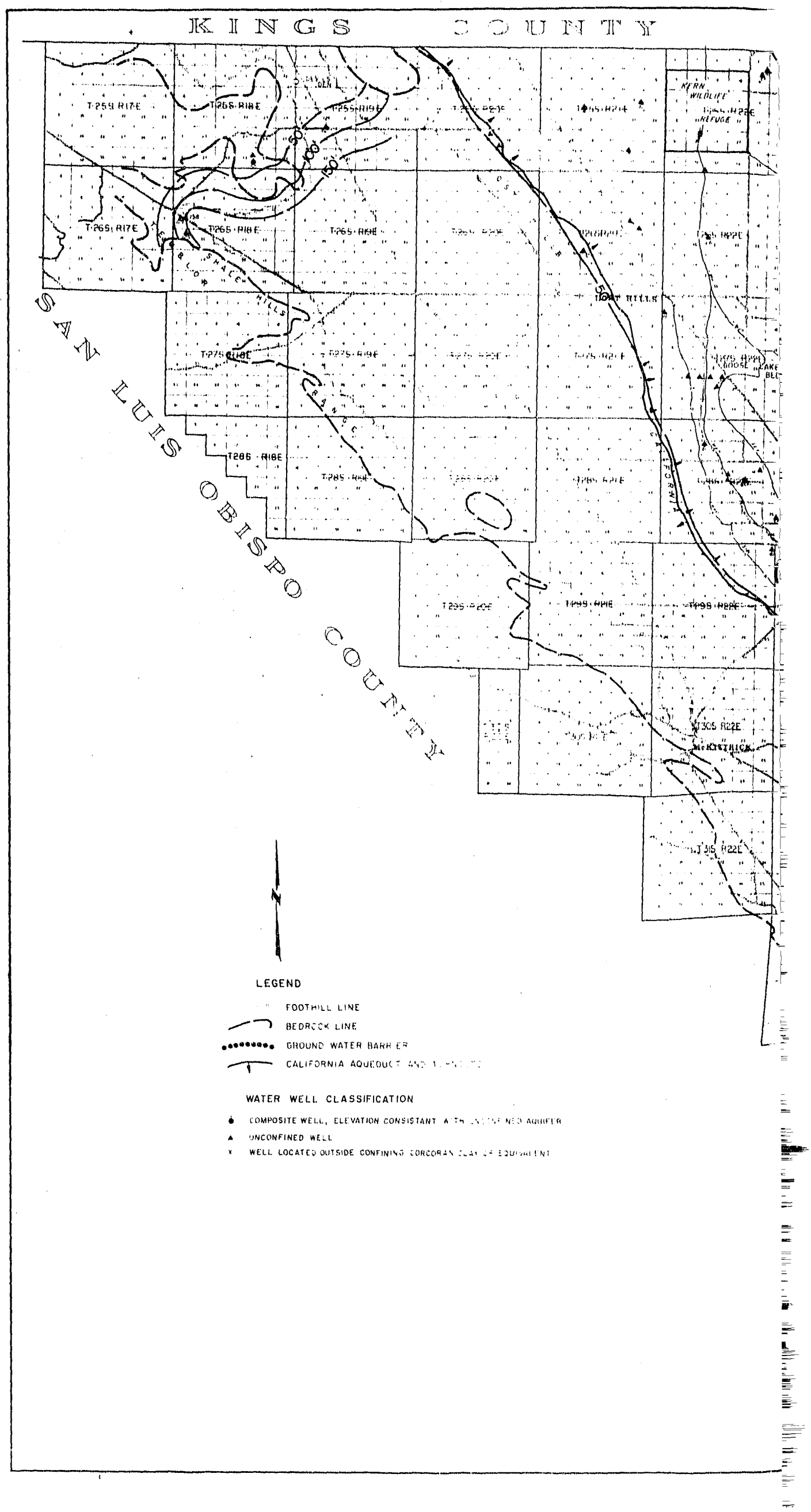


PI_ATE 4

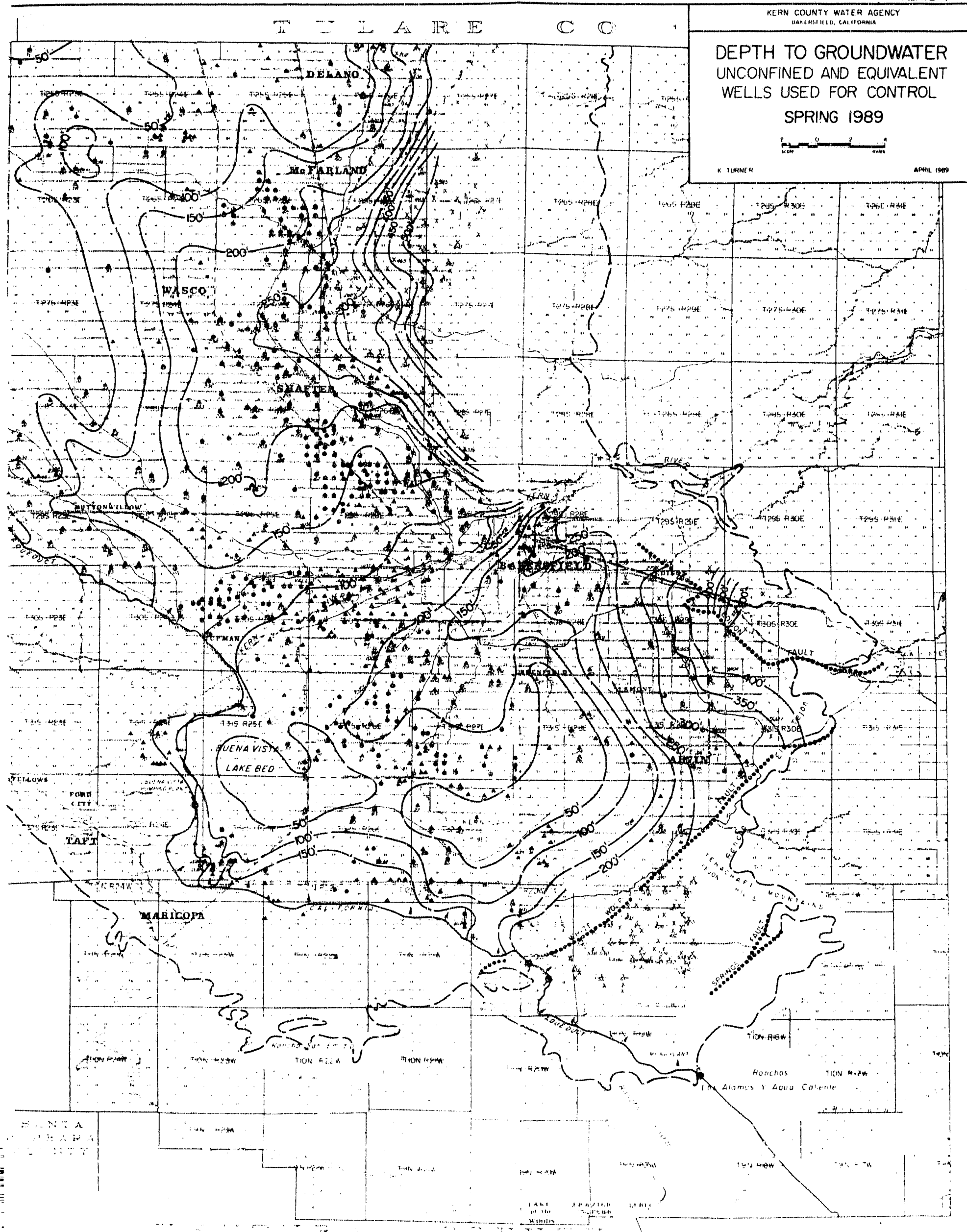




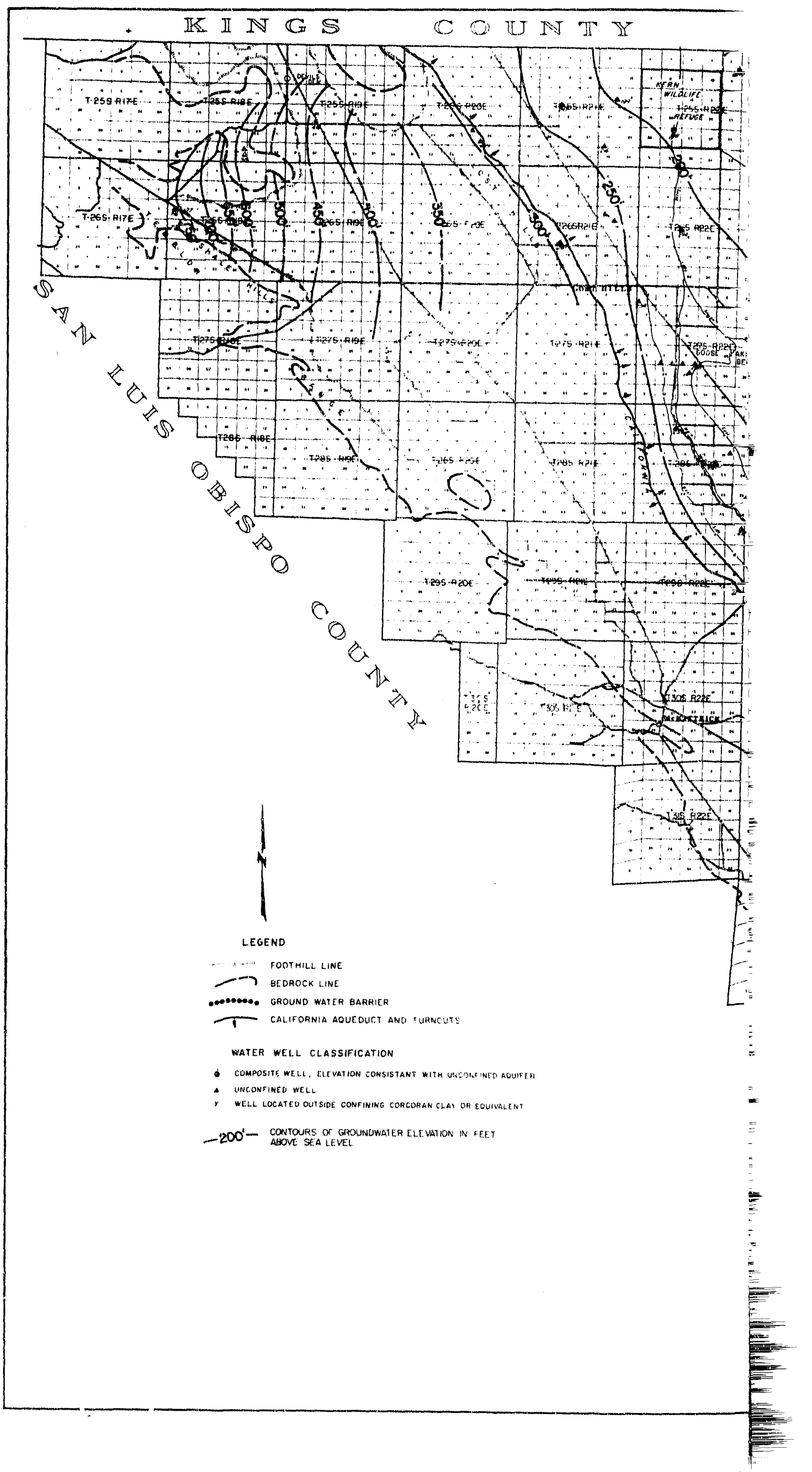




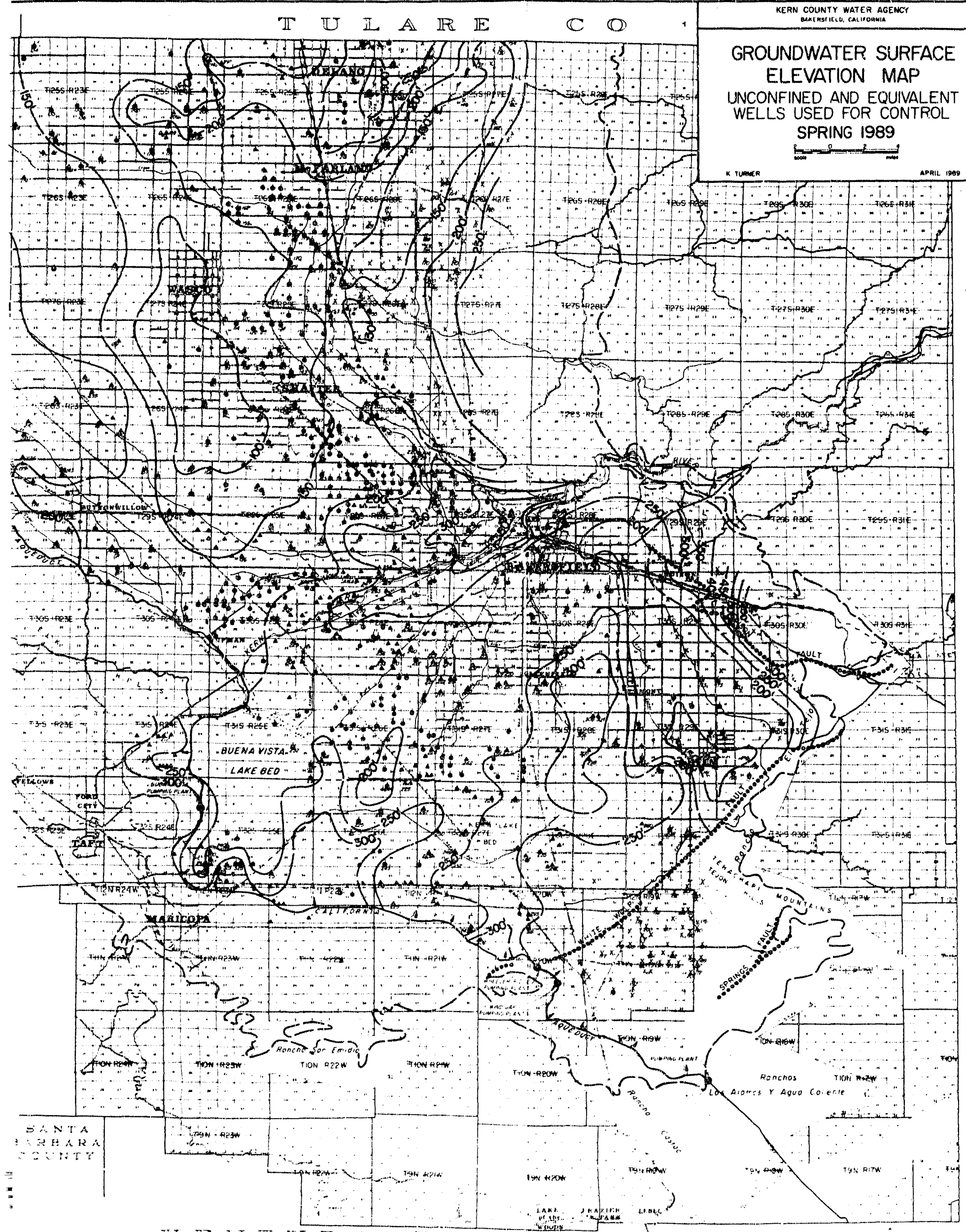




\section{APPENDIX K}

\section{COMPLIANCE ISSUES}




\section{TABLE OF CONTENTS}

\section{APPENDIX K}

1. California Division of Oil and Gas Compliance Issues

II. EPA Notice of Violation and Compliance Agreement

III. Kern County Air Pollution Control District Compliance Issues 


\section{California Division of Oil and Gas Compliance Issues}

- DOG Notice

Date

$12 / 6 / 88$

02/06/89

$10 / 26 / 89$

$10 / 26 / 89$
Deficiency

Packer leak in Well 42-16G

Packer leak in Well 355-4G

Improper Abandonment Well 76-28S

Improper Abandonment Well $52-4 \mathrm{G}$
Corrective Action

Remedial Action letter 2/10/89

Resolved in letter 03/03/89 "Top Perforation Leak, Well 355-4G"

Remedial Action Letter 11/13/89

Remedial Action Letter 11/13/89 


\section{EPA Notice of Violation and Compliance Agreement}

\section{NOx Reduction Program}

On February 22, 1982, the Environmental Protection Agency (EPA) issued a Prevention of Significant Deterioration (PSD) permit to Elk Hills Naval Petroleurn Reserve No. 1 for the installation of 34 large internal combustion engines. Special Condition IX.A of this permit includes specific NOx emission rates of $11 \mathrm{gm} / \mathrm{bhp}$-hr for the 15 Ingersoll-Rand engines, and $10 \mathrm{gm} / \mathrm{bhp}$-hr for the 19 Waukesha engines. On May 16, 1986, EPA issued a notice of violation to NPR-1 for exceedances of these PSD NOx emission limits. To address this problem an engineering study was conducted and finalized in September of 1986. The installation of precombustion chamber (PCC) and pre-stratified charge (PSC) NOx control technologies on the Ingersoll-Rand and Waukesha engines, respectively, was discussed and agreed with EPA Region IX staff as a resolution of NPR-1s compliance status. A Federal Facilities Compliance Agreement was executed by DOE on $2 / 4 / 88$. This Agreement required that:

1. The allowable NOx emissions for the Ingersoll-Rand engines be reduced from $11 \mathrm{gm} / \mathrm{bhp}-\mathrm{hr}$ to $7 \mathrm{gm} / \mathrm{bhp}-\mathrm{hr}$.

2. Final installation of the NOx control technologies on the Ingersoll-Rand and Waukesha engines be completed by $2 / 22 / 89$.

All project milestones were met and final compliance with the Agreement and the new PSD emission limits have been achieved ahead of schedule. Work on the modifications was completed on February 6, 1989. Complinace tests to document emissions were completed on February 7, 1989.

The Federal Facilities Complinace Agreement was formally closed with the issuance of the revised PSD permit. The revised permit was issued on April 7, 1989. 


\section{KERN COUNTY AIR POLLUTION CONTROL DISTRICT COMPLIANCE ISSUES}

\begin{tabular}{lll}
$\begin{array}{c}\text { Date of } \\
\text { Breakdown }\end{array}$ & \multicolumn{1}{c}{ Deficiency } & \multicolumn{1}{c}{ Corrective Action } \\
$01 / 20 / 89$ & $\begin{array}{l}\text { Smoking of LTS-I } \\
\text { HP and LP Flare }\end{array}$ & $01 / 20$ Production Shut-In \\
& Smoking LTS-II & $03 / 06 / 89$ Auxiliary Fans Repaired \\
$03 / 06 / 89$ & LP Flare & \\
& Unit K40 Failed Source & 90-Day Variance \\
$04 / 07 / 89$ & Test & \\
& Smoking LTS-II & 05/24/89 Gas Sensor Replaced \\
$05 / 24 / 89$ & HP Flare & \\
& Smoking LTS-I & 06/22/89 Production Shut-In \\
$06 / 22 / 89$ & LP Flare & Repaired Bad Compressor Valve \\
& Smoking LTS-I \& LTS- & Flare Tips Replaced During October and \\
$06 / 23 / 89$ & II Flares & November 1989 \\
& & Plant Turnaround
\end{tabular}




\section{APPENDIX L}

SUMMARY OF NPR-1 ENVIRONMENTAL PERMIT'S

AND OTHER AGREEMENTS/FINDINGS 


\section{TABLE OF CONTENTS \\ APPENDIX L}

I. Kern County Air Pollution Control District NPR-1 Permits to Operate - 1989

11. Kern County Air Pollution Control District Authorities to Construct - 1989

III. U.S. Fish and Wildlife Service Permits and Biological Opinions

IV. Kern County Health Department (KCHD) Water and Waste Permits

V. California Department of Fish and Game Permits and Memorandums of Understanding 
1. Kern County Air Pollution Contol District NPR-1 Permits to Operate - 1989

\begin{tabular}{|c|c|c|c|c|c|c|}
\hline $\begin{array}{l}\text { PERMIT } \\
\text { MUAUBER } \\
\text { =Ex=E=am= }\end{array}$ & 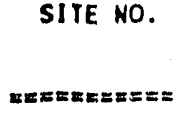 & $\begin{array}{l}\text { OPERATIONAL } \\
\text { DESCRIPIIOW } \\
=x=m=m=m=m\end{array}$ & 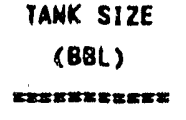 & 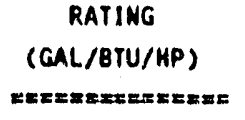 & $=z=x=x=2 z$ & 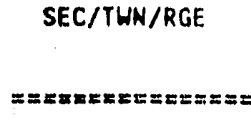 \\
\hline 4091001 & 186 & F/R TAMK & 250000 & 10500000 & 19401 & $18 G / 315 / 24 E$ \\
\hline 4091002 & 186 & F/R TAMK & 250000 & 10500000 & 18402 & $186 / 315 / 24 E$ \\
\hline 4091003 & 186 & F/R TAMK & 250000 & 10500000 & 19603 & $180 / 315 / 24 E$ \\
\hline 4091004 & $18 G$ & F/R TANK & 250000 & 10500000 & 19404 & $186 / 315 / 24 E$ \\
\hline 4091005 & 186 & F/R TANK & 250000 & 10500000 & 19405 & $186 / 315 / 24 E$ \\
\hline 4091007 & LTS-2 & 2-27.5 HEATERS & & 55000000 & & $35 R / 30 S / 23 E$ \\
\hline $4091008 \mathrm{D}$ & 242 & LOADOUT/FLARE & & 4234000 & & $242 / 30 S / 22 E$ \\
\hline 4091009 & L.TS-1 & $2-27.5$ HEATERS & & 55000000 & & $35 R / 30 S / 23 E$ \\
\hline 4091010 & $35 ?$ & TRUCK LOADOUT & 8572 & 360024 & 12954 & $35 R / 30 S / 23 E$ \\
\hline 4091010 & $35 R$ & TRIKCK LONDOUT & & & 12955 & $35 R / 30 S / 23 E$ \\
\hline 4091010 & $35 R$ & TRUCK LOADOUT & & & 12956 & $35 R / 30 S / 23 E$ \\
\hline 4091010 & $35 R$ & TRUCK LOADOUT & & & 12966 & $35 R / 30 S / 23 E$ \\
\hline 4001011 & $35 R$ & TRUCK LOADOUT & 1400 & 58800 & 11747 & $35 R / 30 S / 23 E$ \\
\hline 4091300 & $35 R$ & TRUCK LONOOUT & 1400 & 58800 & 11746 & $35 R / 30 S / 23 E$ \\
\hline 6091301 & $35 R$ & TRUCK LONDOUT & 1400 & 58800 & 9031 & $35 R / 30 S / 23 E$ \\
\hline 4091302 & $35 R$ & TRUOX LOADOUT & 1400 & 58800 & 9030 & $35 R / 30 S / 23 E$ \\
\hline 40910148 & 368 & IC EMGINE RG & & 1000 & & SSR/3OS/23E \\
\hline 4091015 & $35 R$ & LTS-2 HP FLARE & & 999999999.99 & & $35 R / 30 S / 23 E$ \\
\hline 4091016 & $35 R$ & LTS-2 LP FLARE & & 1046000000 & & $35 R / 30 S / 23 E$ \\
\hline 4091017 & $35 R$ & IC ENGINE K40 & & 5500 & 16652 & $35 R / 30 S / 23 E$ \\
\hline $4091018 A$ & $35 R$ & IC EMGIHE XA 1 & & 5500 & 16651 & $35 R / 30 S / 23 E$ \\
\hline 4091049 & $35 R$ & IC ENGIME K 42 & & 5500 & 16650 & $35 R / 30 S / 23 E$ \\
\hline $4091020 A$ & $35 R$ & IC EMGINE K 43 & & 2000 & 13694 & $35 R / 30 S / 23 E$ \\
\hline 4091021 & $35 R$ & LTS-JMP FLARE & & 999999099.99 & & $35 R / 30 S / 23 E$ \\
\hline 4091022 & $35 R$ & LTS-ILP FLARE & & 1056000000 & & 35R/30S/23E \\
\hline 4091023 & $35 R$ & IC EMGIME $\times 36$ & & 5500 & 13675 & $35 R / 30 S / 23 E$ \\
\hline $4091026 A$ & $35 R$ & IC EMGIME KOT & & 5500 & 13674 & $35 R / 30 S / 23 E$ \\
\hline 4091025 & $35 R$ & IC EMGIME $X 38$ & & 5500 & 13673 & $35 R / 30 S / 23 E$ \\
\hline $4091026 A$ & $35 R$ & IC EMGIME KOSQ & & 2000 & 13672 & $35 R / 30 S / 23 E$ \\
\hline $4091030 \AA$ & $30 k$ & IC EMGINE K53 & & 1000 & 15672 & $30 R / 30 S / 23 E$ \\
\hline 4091031 B & $17 R$ & IC EMGIME K4Q & & 1000 & 51286 & $17 R / 30 S / 23 F$ \\
\hline $4091032 A$ & $30 R$ & IC EMGIME K2O & & 1000 & 13401 & $30 R / 30 S / 23 E$ \\
\hline 4091033 & $33 R$ & IC EMGIME KS4 & & 650 & 12493 & $33 R / 30 S / 23 E$ \\
\hline $4091034 A$ & $36 R$ & IC EMGINE KSZ & & 1000 & 18028 & $36 R / 30 S / 23 E$ \\
\hline $4091036 A$ & 335 & IC EMGINE XW4 & & 2000 & 13701 & $33 S / 30 S / 24 E$ \\
\hline $4091037 A$ & 335 & IC EMGIME $\times 48$ & & 1000 & $12 a n$ & $33 S / 30 S / 24 E$ \\
\hline 4001038 & $35 R$ & IC EMGIME K23 & & 4000 & 13676 & $35 R / 30 S / 23 E$ \\
\hline 4091040 & $35 R$ & 35R CP MEATERS & & 63147500 & & $35 R / 30 S / 23 E$ \\
\hline 4091069 & $35 R$ & IC EMGINE KI & & 880 & 9071 & $35 R / 30 S / 23 E$ \\
\hline 4091042 & $35 R$ & IC EMGIME K2 & & 880 & 9066 & $35 R / 30 S / 23 E$ \\
\hline 4091043 & 35R & IC EHGINE KS & & 880 & 9149 & $35 R / 30 S / 23 E$ \\
\hline
\end{tabular}


1. Kern County Air Pollution Contol District NPR-1 Permits to Operate - 1989

\begin{tabular}{|c|c|c|c|c|c|c|}
\hline $\begin{array}{l}\text { PERMIT } \\
\text { NIMABER } \\
=x=2=x=x=\end{array}$ & 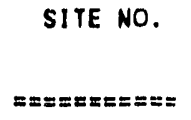 & $\begin{array}{l}\text { OPERATIONAL } \\
\text { DESCRIPTION } \\
=x=x=m=m=m=m=x\end{array}$ & $\begin{array}{c}\text { TANK SIZE } \\
\text { (BBL) } \\
===========\end{array}$ & $\begin{array}{c}\text { RATING } \\
(\text { GAL/BTU/HP) } \\
======= \pm==2= \pm= \pm=\end{array}$ & $== \pm=8 \pi 8 \pi 5 \pi$ & 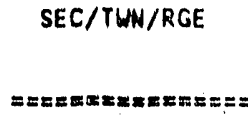 \\
\hline 4081044 & $35 R$ & IC EMGINE KG & & 880 & 9154 & $35 R / 30 S / 23 E$ \\
\hline 4091045 & $35 R$ & IC ENGINE KS & & 880 & 9159 & $35 R / 30 S / 23 E$ \\
\hline 4091046 & $35 R$ & IC EMGINE KG & & 880 & 9164 & $35 R / 30 S / 23 E$ \\
\hline 4091047 & $35 R$ & IC ENGIAE $K 7$ & & 880 & 9169 & $35 R / 30 S / 23 E$ \\
\hline 4091048 & $35 R$ & IC ËMGINE K9 & & 4000 & 14726 & $35 R / 30 S / 23 E$ \\
\hline 4091049 & $35 R$ & IC ENGINE K10 & & 4000 & 11718 & $35 R / 30 S / 23 E$ \\
\hline $4091050 \mathrm{~A}$ & $35 R$ & IC ENGINE K11 & & 1000 & 50817 & $35 R / 30 S / 23 E$ \\
\hline $4091051 \mathrm{~A}$ & $35 R$ & IC EMGINE K12 & & 1000 & 13417 & $35 R / 30 S / 23 E$ \\
\hline $4091052 A$ & $35 R$ & IC EMGINE K13 & & 1000 & 18033 & $35 R / 30 S / 23 E$ \\
\hline 4091053 & $35 R$ & 35R GP HEATERS & & 55650000 & F204 & $35 R / 30 S / 23 E$ \\
\hline 4091055 & $35 R$ & 35R GP HEATERS & & 55650000 & F205 & $35 R / 30 S / 23 E$ \\
\hline 4091057 & $17 R$ & IC ENGINE K26 & & 650 & 11554 & $17 R / 30 S / 23 E$ \\
\hline $4091058 \mathrm{~A}$ & $17 R$ & IC ENGINE K27 & & 650 & 12705 & $17 R / 30 S / 23 E$ \\
\hline $4091059 \mathrm{~A}$ & $17 R$ & IC ENGINE K28 & & 650 & 12850 & $17 R / 30 S / 23 E$ \\
\hline $409+r_{1}: 50 A$ & $30 R$ & IC EAGINE K3O & & 1000 & 11563 & $30 R / 30 S / 23 E$ \\
\hline $4091061 \mathrm{~A}$ & 30R & IC ENGINE K31 & & 1000 & 13426 & 3OR/3OS/23E \\
\hline $4091062 A$ & $30 R$ & IC ENGINE K32 & & 1000 & 13198 & $30 R / 30 S / 23 E$ \\
\hline $4091063 \mathrm{~A}$ & SOR & IC ENGINE K33 & & 1000 & 12877 & 3OR/3OS/23E \\
\hline $4091064 \mathrm{~A}$ & 3OR & IC ENGINE K34 & & 1000 & 15676 & $30 R / 30 S / 23 E$ \\
\hline $4091065 \mathrm{~A}$ & $30 R$ & IC ENGINE K35 & & 1000 & 13207 & $30 R / 30 S / 23 E$ \\
\hline $4091066 \mathrm{~A}$ & $36 R$ & IC ENGINE KSI & & 1000 & 318898 & $36 R / 30 S / 23 E$ \\
\hline $4091067 \mathrm{~A}$ & $36 R$ & IC ENGIAE K5O & & 1000 & 16453 & $36 R / 30 S / 23 E$ \\
\hline $4091068 A$ & $33 \mathrm{~s}$ & IC ENGINE K45 & & 2000 & 13706 & $33 S / 30 S / 24 E$ \\
\hline 4091069 & $33 s$ & IC ENGINE K46 & & 2000 & 13715 & $33 S / 30 S / 24 E$ \\
\hline 4091070 & $33 \mathrm{~s}$ & IC ENGINE $K 47$ & & 2000 & 13716 & $33 S / 30 S / 24 E$ \\
\hline $4091071 A$ & $35 R$ & IC ENGINE K24 & & 4000 & 13677 & $35 R / 30 S / 23 E$ \\
\hline 4091072 & $35 R$ & IC ENGINE $\times 23$ & & 4000 & 13678 & $35 R / 305 / 23 E$ \\
\hline 4091073 & $7 R$ & IC ENGINE KSB & & 1000 & 13444 & $7 R / 30 S / 23 E$ \\
\hline 4091074 & $7 R$ & IC ENGINE KST & & 1000 & 18017 & $\pi R / 30 S / 23 E$ \\
\hline 4091075 & $36 R$ & IC ENGINE KSO & & 1000 & 53881 & $36 R / 30 S / 23 E$ \\
\hline 4091076 & $36 R$ & IC ENGINE KGO & & 1000 & 53868 & $36 R / 30 S / 23 E$ \\
\hline 4091100 & $3-33 s$ & TANK FACILITY & 500 & 21000 & 1165 & $33 S / 30 S / 24 E$ \\
\hline 4091303 & $3.33 s$ & TAHK FACILITY & 500 & 21000 & 1192 & $335 / 305 / 24 E$ \\
\hline 4091489 & $3-33 s$ & TANK FACILITY & 35 & 1470 & 19367 & $335 / 30 S / 24 E$ \\
\hline 4091101 & $2-46$ & TANK FACILITY & 1000 & 42000 & 1004 & $2 G / 30 S / 24 E$ \\
\hline 4091304 & $2-4 G$ & TANK FACILITY & 1000 & 42000 & 1098 & $2 G / 30 S / 24 E$ \\
\hline 4091305 & 2.40 & TANK FACILITY & 500 & 21000 & 1005 & $2 G / 30 S / 24 E$ \\
\hline 4091482 & $2 \cdot 40$ & TANK FACILITY & 35 & 1470 & 19365 & $2 G / 30 S / 24 E$ \\
\hline 4091102 & $4-345$ & TANK FACILITY & 1000 & 42000 & 1003 & $345 / 30 S / 24 \mathrm{E}$ \\
\hline 4091306 & $4-345$ & TANK FACILITY & 500 & 21000 & 1002 & $365 / 30 S / 24 E$ \\
\hline 4091307 & $4-345$ & TANK FACILITY & 500 & 21000 & 1237 & $345 / 30 S / 24 E$ \\
\hline
\end{tabular}


1. Kern County Air Pollution Contol District NPR. 1 Permits to Operate - 1989

\begin{tabular}{|c|c|c|c|c|c|c|c|}
\hline $\begin{array}{l}\text { PF.RMIT } \\
\text { NUMBER } \\
=m= \pm=x==\end{array}$ & $==x=x=2=x==$ & $\begin{array}{l}\text { OPER } \\
\text { DESC } \\
== \pm=2\end{array}$ & $\begin{array}{l}\text { RAIIONAL } \\
\text { CRIPYION } \\
=x=x=x=x=x\end{array}$ & $\begin{array}{c}\text { TANK SIZE } \\
\text { (BBL) } \\
==x=x=m=x=\pi x\end{array}$ & $\begin{array}{c}\text { RATING } \\
\text { (GAL/BTU/HP) } \\
==========m====\end{array}$ & $z==2=m=x=$ & 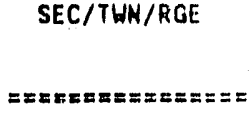 \\
\hline 4091483 & $4-34 S$ & TANK & FACILITY & 35 & 1470 & 19380 & $34 S / 30 S / 24 E$ \\
\hline 4001103 & $3-35 s$ & TANK & FACILITY & 2000 & 84000 & 1078 & $35 s / 30 S / 24 E$ \\
\hline 4091308 & $3-35 s$ & TANK & FACILITY & 1000 & 42000 & 1031 & $35 S / 30 S / 24 E$ \\
\hline 4091309 & $3-35 s$ & TANK & FACILITY & 500 & 21000 & $10 \pi$ & 35S/30S/24E \\
\hline 4081310 & $3-35 s$ & TANK & FACILITY & 500 & 21000 & 1148 & $35 S / 30 S / 24 E$ \\
\hline 4091311 & $3-35 s$ & TANK & FACILITY & 500 & 21000 & 1146 & $35 S / 30 S / 24 E$ \\
\hline 4091484 & $3-35 s$ & TANK & FACILITY & 35 & 1470 & 19364 & $35 S / 30 S / 24 E$ \\
\hline 4091104 & $1-25 R$ & TANK & FACILITY & 1000 & 42000 & 1033 & $25 R / 30 S / 23 E$ \\
\hline 4091312 & $1-25 R$ & TANK & FACILITY & 500 & 21000 & 1032 & $25 R / 30 S / 23 E$ \\
\hline 4091485 & $1-25 R$ & TANK & FACILITY & 35 & 1470 & 19858 & $25 R / 30 S / 23 E$ \\
\hline 4091105 & $3-25 R$ & TANK & FACILITY & 1000 & 42000 & 1012 & $25 R / 30 S / 23 E$ \\
\hline 4091313 & $3-25 R$ & TANK & FACILITY & 500 & 21000 & 1013 & $25 R / 30 S / 23 E$ \\
\hline 4091314 & $3-25 R$ & TANK & FACILITY & 500 & 21000 & 1134 & $25 R / 30 S / 23 E$ \\
\hline 4091486 & $3-25 R$ & TAKK & FACILITY & 35 & 1470 & 19333 & $25 R / 30 S / 23 E$ \\
\hline 4091106 & $2 \cdot 25 R$ & TANK & FACIIIITY & 500 & 21000 & 1055 & $25 R / 30 S / 23 E$ \\
\hline 4091315 & $2 \cdot 25 R$ & TANK & FACILITY & 500 & 21000 & 1056 & $25 R / 30 S / 23 E$ \\
\hline 4091487 & $2 \cdot 25 R$ & TANK & FACILITY & 35 & 1470 & 19863 & $25 R / 30 S / 23 E$ \\
\hline 4091107 & $4-25 R$ & TANK & FACILITY & 500 & 21000 & 1030 & $25 R / 30 S / 23 E$ \\
\hline 4091316 & $4-25 R$ & TANK & FACILITY & 500 & 21000 & 1161 & $25 R / 30 S / 23 E$ \\
\hline 4091488 & $4-25 R$ & TANK & FACILITY & 35 & 1470 & 19339 & $25 R / 30 S / 23 E$ \\
\hline 4081108 & $2-36 R$ & TANK & FACILITY & 1000 & 42000 & 1067 & $36 R / 30 S / 23 E$ \\
\hline 4091317 & $2-36 R$ & TANK & FACILITY & 500 & 21000 & 1066 & $36 R / 305 / 23 E$ \\
\hline 4091318 & $2-36 R$ & TANK & FACILITY & 500 & 21000 & 1163 & $36 R / 30 S / 23 E$ \\
\hline 4091319 & $2-36 R$ & TANK & FACILITY & 100 & 4200 & 19360 & $36 R / 30 S / 23 E$ \\
\hline 4091489 & $2 \cdot 36 R$ & TANK & FACILIIY & 35 & 1470 & 19358 & $36 R / 30 S / 23 \mathrm{E}$ \\
\hline 4091109 & $3-30 s$ & TANK & FACILITY & 500 & 21000 & 1059 & $30 S / 30 S / 24 \mathrm{E}$ \\
\hline 4091320 & $3-30 S$ & TANK & FACILITY & 500 & 21000 & 1060 & $30 \mathrm{~S} / 30 \mathrm{~S} / 24 \mathrm{E}$ \\
\hline 4091490 & $3-30 s$ & TANK & FACILITY & 35 & 1670 & 11386 & $30 \mathrm{~S} / 30 \mathrm{~S} / 24 \mathrm{E}$ \\
\hline 4091491 & $3-30 S$ & TANK & FACILITY & 35 & 1470 & 19329 & $30 S / 30 S / 24 E$ \\
\hline 4091110 & $1-315$ & TANK & FACILITY & 500 & 21000 & 1049 & $315 / 30 S / 24 E$ \\
\hline 4091321 & $1-315$ & TAHK & FACILITY & 500 & 21000 & 1042 & $31 S / 30 S / 24 E$ \\
\hline 4091492 & 1.315 & TANK & FACILITY & 35 & 1470 & 19389 & $315 / 30 S / 24 E$ \\
\hline 4091111 & $3-315$ & TANK & PACILITY & 500 & 21000 & 1132 & $31 S / 30 S / 24 E$ \\
\hline 4091322 & $3-315$ & TANK & FACILITY & 500 & 21000 & 1024 & $315 / 30 S / 24 E$ \\
\hline 4091323 & $3 \cdot 315$ & TAAK & FACILITY & 500 & 21000 & 1025 & $315 / 30 S / 24 E$ \\
\hline 4091493 & $3-31 s$ & TANK & FACILITY & 35 & 1470 & 19400 & $315 / 30 S / 24 E$ \\
\hline 40919128 & $2-315$ & TANK & FACILITY & 500 & 21000 & 1053 & $315 / 30 S / 24 E$ \\
\hline 4091324 & $2-315$ & TANK & FACILITY & 500 & 21000 & 1054 & $315 / 30 S / 24 E$ \\
\hline 4091494 & $2-315$ & TANK & FACILITY & 35 & 1470 & 19386 & $315 / 30 S / 24 E$ \\
\hline $4091113 \mathrm{~B}$ & $9-325$ & TANK & FACILITY & 500 & 21000 & 1006 & $325 / 30 S / 24 \mathrm{E}$ \\
\hline 4091325 & $1-325$ & TANK & FACILITY & 500 & 21000 & 1007 & $32 S / 30 S / 24 E$ \\
\hline
\end{tabular}


1. Kern County Air Pollution Contol District

NPR-1 Permits to Operate . 1989

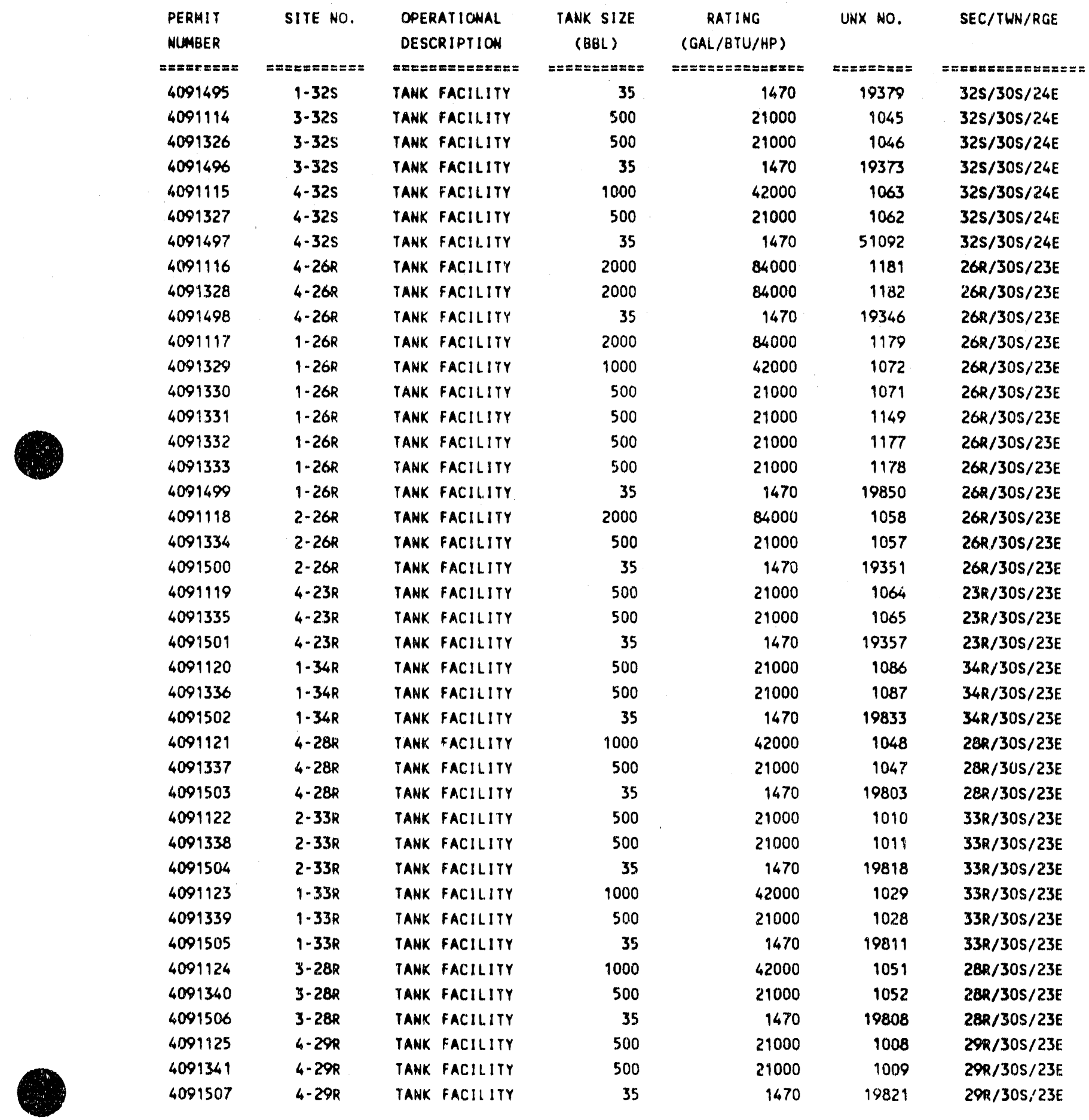


1. Kern County Air Pollution Contol Disitict NPR-1 Permits to Operate. 1989

\begin{tabular}{|c|c|c|c|c|c|c|c|}
\hline PEQAIT & TE NO. & \multirow{2}{*}{\multicolumn{2}{|c|}{$\begin{array}{l}\text { OPERAT . XNAL } \\
\text { DESCRIPTION }\end{array}$}} & TANK SIZE & & UNX NO. & SEC/TWN/RGE \\
\hline NUMEER & & & & (BBL) & (CAL/BTU/HP) & & \\
\hline$=x \operatorname{mex} x \operatorname{sex}$ & 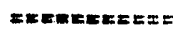 & \multicolumn{2}{|c|}{ 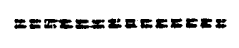 } & 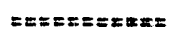 & 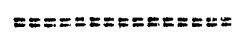 & $==z=x=\pi x z$ & 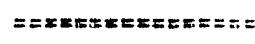 \\
\hline 6091126 & $2-290$ & TAMK & FACILITY & 500 & 21000 & 1144 & $20 R / 305 / 23 E$ \\
\hline 4001342 & $2-29 R$ & TAME & FACILITY & 500 & 21000 & 1038 & $29 R / 305 / 23 E$ \\
\hline 4094508 & $2 \cdot 29 R$ & TANK & FACILITY & 35 & 1470 & 19842 & $29 R / 30 S / 23 E$ \\
\hline 6081127 & $1-29 R$ & TANK & FACILITY & 1000 & 42000 & 1040 & $292 / 30 S / 23 E$ \\
\hline 6001343 & $1-298$ & TANK & FACILITY & 500 & 28000 & 1039 & $29 R / 30 S / 2.3 E$ \\
\hline 4001509 & $1.24 R$ & TANKK & FACILIYY & 35 & 1470 & 51089 & $29 R / 30 S / 23 E$ \\
\hline 4091128 & $2 \cdot 30 R$ & TAMK & FACILITY & 500 & 21000 & 1026 & $30 R / 30 S / 23 E$ \\
\hline 4091344 & $2-30 R$ & TANKK & FACILITY & 500 & 21000 & 1027 & $30 R / 30 S / 23 E$ \\
\hline 4091510 & $2-30 R$ & TANK & FACILITY & 35 & 1670 & 19829 & $30 R / 30 S / 23 E$ \\
\hline 10091120 & $4-18 R$ & TANK & FACILITY & 1000 & 42000 & 1089 & $19 R / 30 S / 23 E$ \\
\hline 6091345 & $4-19 R$ & IANK & FACILITY & 500 & 21000 & 1088 & $19 R / 305 / 23 E$ \\
\hline 4091511 & $4-99 R$ & IAWK & FACILITY & 35 & 1470 & 19825 & $19 R / 30 S / 23 E$ \\
\hline 4091130 & $3-19 R$ & TANK & FACILITY & 500 & 21000 & 1104 & $19 R / 30 S / 23 E$ \\
\hline 4091346 & $3-19 R$ & TANK & FACILITY & 500 & 21000 & 9271 & $19 R / 30 S / 23 E$ \\
\hline 6091512 & $3-19 R$ & TiNh & YACILITY & 35 & 1470 & 19854 & $19 R / 303 / 23 E$ \\
\hline 4001131 & $1 \cdot 262$ & TANK & FACILITY & 1000 & 42000 & 1001 & $242 / 20 S / 22 E$ \\
\hline 4001347 & $1-242$ & TAHK & FACILITY & 1000 & 42000 & 1037 & $262 / 30 \mathrm{~S} / 22 \mathrm{E}$ \\
\hline 4001513 & $1-242$ & TANK & FACILITY & 35 & 1470 & 51083 & $242 / 30 S / 22 E$ \\
\hline 4001133 & $3-15 R$ & TANK & FACILITY & $2 r, 20$ & 84000 & 53130 & $152 / .05 / 23 E$ \\
\hline 4099348 & $3.15 \pi$ & TANK & FACILITY & 500 & 21000 & 1196 & $15 R / 30 S / 23 E$ \\
\hline 4091134 & $4-16 R$ & TANK & FACILITY & 1000 & 42000 & 1158 & $96 R / 30 S / 23 E$ \\
\hline 4091350 & $4-16 R$ & TANK & FACILITY & 1000 & 42000 & 1197 & $16 R / 30 S / 23 E$ \\
\hline 4001351 & $4-16 R$ & TAHK & FACILITY & 35 & 1470 & 16211 & $96 R / 305 / 23 E$ \\
\hline 40911358 & $4-3 G$ & TANK & FACILITY & 500 & 21000 & 1124 & $3 G / 315 / 24 E$ \\
\hline 4081399 & $4-30$ & TANK & FACILITY & 500 & 21000 & 1123 & $36 / 315 / 24 E$ \\
\hline 4001352 & $4-36$ & TANK & FACILIYY & 100 & 4200 & 50740 & $36 / 315 / 24 E$ \\
\hline 4091136 & $1 \cdot 7 R$ & TANK & FACRLITY & 2000 & 84000 & 50296 & $7 R / 30 s / 23 E$ \\
\hline 4081401 & $1-7 R$ & TANK & FACILITY & 2000 & 84000 & 50297 & $\pi / 30 s / 23 E$ \\
\hline 6099602 & $1-72$ & TANK & FACILITY & Tso & 31500 & 50295 & $T R / 30 S / 23 E$ \\
\hline 4001531 & $1-7 R$ & TANK & FACILI: & 500 & 21000 & 15623 & TR/30S/2Li \\
\hline 4091137 & 242 DEHY & TAMK & FACII, TY & 16000 & 672.000 & 11468 & $242.305 / 22 \mathrm{E}$ \\
\hline 4091403 & 242 DEKY & TANK & FACYIITY & 16000 & 672000 & 11469 & $2420 \mathrm{OS} / 22 \mathrm{E}$ \\
\hline 4099604 & $24 Z$ DEHY & TANK & FACILITT & 16000 & 672000 & 11470 & $242 / 30 S / 22 E$ \\
\hline 6.091605 & ZLZ DEKY & TANK & FACILITY & 16000 & 672000 & 50539 & $242 / 30 S / 22 E$ \\
\hline & 242 DEHT & TANK & FACILITY & 5000 & 210000 & 10390 & $242 / 305 / 22 E$ \\
\hline & $24 Z$ DE:HY & TAKK & FACILITY & 5000 & 210000 & 1285 & $242 / 30 S / 22 E$ \\
\hline & 242 DEHY & TANK & FACILITY & 500 & 2.1000 & 1140 & $242 / 30 S / 22 E$ \\
\hline 4001138 & $4-242$ & TANK & FACILI:: & 500 & 21000 & 14218 & $262 / 305 / 22 E$ \\
\hline 4001606 & $4-242$ & TANK & faCilitr & 2000 & $841: 00$ & 14216 & $242 / 305 / 22 E$ \\
\hline 4091407 & $4 \cdot 242$ & TAMK & FACILITY & 2000 & 84000 & 14217 & $242 / 30 S / 22 E$ \\
\hline 6091532 & $6-262$ & TANK & FACILITY & 900 & 4200 & 14258 & $242 / 30 S / 22 \mathrm{E}$ \\
\hline
\end{tabular}


1. Kern County Air Pollutic 1 Contol District

NPR- 9 Permits to Operate - 1989

\begin{tabular}{|c|c|c|c|c|c|c|c|}
\hline $\begin{array}{l}\text { PERMIT } \\
\text { MUAABER } \\
x=\pi=x=2=5=\end{array}$ & 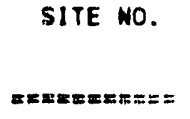 & $\begin{array}{l}\text { OPER } \\
\text { DESC } \\
=\pi==x\end{array}$ & $\begin{array}{l}\text { SATIONAL } \\
\text { CRIPTION } \\
==x==x=x=2\end{array}$ & $\begin{array}{l}\text { TANK SIZE } \\
\text { (BBL) } \\
x=x= \pm=m=2==\end{array}$ & $\begin{array}{c}\text { RATING } \\
\text { (GAL/BTU/HP) } \\
=========\pi=====\end{array}$ & $=2 x=x=2 x=0$ & 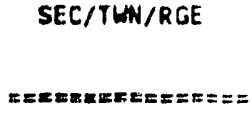 \\
\hline 4091139 & $4-242$ & TANK & FACILITY & 500 & 29000 & 1070 & $242 / 30 S / 22 E$ \\
\hline 4091140 & $2-3 G$ & TANK & FACILITY & 1000 & 42000 & 1104 & $3 G / 315 / 24 E$ \\
\hline 4091409 & $2-36$ & TANK & FACILITY & 500 & 21000 & 1103 & $3 G / 31 S / 24 E$ \\
\hline 4091533 & $2 \cdot 36$ & TANK & FACILITY & 35 & 1470 & 14958 & $30 / 315 / 24 E$ \\
\hline 4091534 & $2-36$ & TANK & FACILITY & 35 & 1470 & 19528 & $30 / 315 / 24 E$ \\
\hline 4091149 & $1-3 G$ & TANK & FACILITY & 1000 & 42000 & 1034 & $3 G / 31 S / 24 E$ \\
\hline 4099410 & $1-36$ & TANK & FACILITY & 500 & 21000 & 1231 & $36 / 315 / 24 E$ \\
\hline 4091535 & $9 \cdot 36$ & TANK & FACILITY & 35 & 1470 & 14958 & $3 G / 315 / 24 E$ \\
\hline 4091142 & $3-345$ & TAMK & FACILITY & 1000 & 42000 & 1931 & $345 / 30 S / 24 E$ \\
\hline 4091491 & $3-345$ & TANK & FACILITY & 500 & 21000 & 1930 & $345 / 30 S / 24 E$ \\
\hline 4091412 & 3.345 & TANK & FACILITY & 500 & 21000 & 1138 & $345 / 30 S / 24 E$ \\
\hline 4001536 & $3-365$ & TANK & FACILITY & 35 & 1470 & 16957 & $345 / 30 S / 24 E$ \\
\hline 4091143 & $4-335$ & TANK & FACILITY & 500 & 21000 & 1020 & $335 / 305 / 24 E$ \\
\hline 4091413 & $4-33 s$ & TANK & FACILITY & 1000 & 42000 & 1021 & $335 / 30 S / 24 E$ \\
\hline 4094537 & $4-335$ & TANK & FACILITY & 35 & 1470 & 14956 & $335 / 30 S / 24 E$ \\
\hline 4081144 & $1-40$ & TANK & FACILITY & 1000 & 42000 & 1080 & $4 G / 315 / 24 E$ \\
\hline 4099414 & $1-46$ & TANK & FACILITY & 1000 & $4 c 000$ & 1014 & $4 G / 315 / 24 E$ \\
\hline 4001415 & $1-46$ & TANK & FACILITY & 500 & 21000 & 1079 & $40 / 315 / 24 E$ \\
\hline 4091538 & $1-46$ & TANK & FACILITY & 35 & 1470 & 14955 & $4 G / 315 / 24 E$ \\
\hline 4091145 & 2.56 & TANK & FACILITY & 500 & 21000 & 1023 & $56 / 31 S / 24 E$ \\
\hline 4091416 & $2-5 G$ & TANK & FACILITY & 500 & 21000 & 1022 & $56 / 315 / 24 E$ \\
\hline 1,091417 & 2.50 & TANK & FACILITY & 500 & 21000 & 1147 & $5 G / 315 / 24 E$ \\
\hline 4091539 & $2 \cdot 56$ & TANK & FACILITY & 35 & 1470 & 14954 & $56 / 31 S / 24 E$ \\
\hline 4091146 & $1-5 G$ & TANK & FACILITY & 500 & 21000 & 1016 & $5 G / 31 S / 24 E$ \\
\hline 4091418 & $1-5 c$ & TANK & FACILITY & 500 & 21000 & 1017 & $50 / 31 S / 24 E$ \\
\hline 4091419 & $1-5 G$ & TANK & FACILITY & 500 & 21000 & 1437 & $5 G / 315 / 24 E$ \\
\hline 4091420 & $1-56$ & TANK & FACILITY & 500 & 21000 & 1015 & $56 / 315 / 24 E$ \\
\hline 4091540 & $1-56$ & TANK & FACILI 1 & 35 & 1470 & 14953 & $50 / 315 / 26 E$ \\
\hline $4091147 \mathrm{C}$ & $6-315$ & TANK & FACILIYY & 500 & 21000 & 1036 & $315 / 30 \mathrm{~S} / 24 \mathrm{E}$ \\
\hline 4091421 & $4 \cdot 315$ & TANK & FACILITY & 500 & 2.1000 & 1035 & $315 / 30 S / 24 E$ \\
\hline 40914.22 & $4-315$ & TAHK & FACILITY & 500 & 21000 & 1133 & $315 / 30 S / 26 E$ \\
\hline 4091541 & 4.315 & TANK & FACILITY & 35 & 1470 & 14952 & $315 / 30 S / 24 E$ \\
\hline 4091148 & $1-26$ & TANK & FACILIYY & 1000 & 42000 & 1049 & $20 / 315 / 24 E$ \\
\hline 4091423 & $1-2, G$ & TANK & FACILITY & 500 & 21000 & 1050 & $2 G / 315 / 24 E$ \\
\hline 4001542 & $1-26$ & TAMK & FACILITY & 35 & 1470 & 16960 & $26 / 315 / 24 E$ \\
\hline 4001543 & $9-26$ & TANK & FACILITY & 35 & 1470 & 19525 & $26 / 315 / 24 E$ \\
\hline 4091149 & $1-36 R$ & TANK & FACILITY & 2000 & 84000 & 1186 & $36 R / 30 S / 23 E$ \\
\hline 4081424 & $1.36 n$ & TANK & FACILITY & 1000 & 42000 & 1090 & $36 k / 30 \mathrm{~S} / 23 \mathrm{E}$ \\
\hline 4091425 & $1-36 R$ & TAWK & FACILITY & 500 & 21000 & 1084 & $36 n / 30 s / 23 E$ \\
\hline 4001926 & i $3 \leqslant n$ & 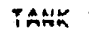 & racilaty & 500 & 29000 & ines & $26, i^{2} 05,23 E$ \\
\hline 4091544 & $1-368$ & TAHK & FACILITYY & 35 & 1470 & 14959 & $36 / 30 S / 23 E$ \\
\hline
\end{tabular}


1. Kern County Air Pollution Contol District

NPR- 1 Permits to Operote - 1989

\begin{tabular}{|c|c|c|c|c|c|c|c|}
\hline $\begin{array}{l}\text { PERMIT } \\
\text { MUMBER }\end{array}$ & SITE NO. & & $\begin{array}{l}\text { RATIONAL } \\
\text { CRIPTION }\end{array}$ & $\begin{array}{l}\text { TANK S:ZE } \\
\text { (BBL) }\end{array}$ & $\begin{array}{c}\text { RATING } \\
\text { (GAL/BTU/HP) }\end{array}$ & UNX NO. & SEC/TWW/RGE \\
\hline 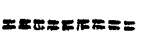 & $==z x=2 x=z==$ & $=x=x$ & $z=2=2 x=2$ & 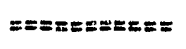 & 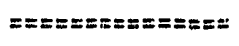 & $=x=2 x=2 \pi$ & 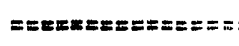 \\
\hline 40911508 & 18G OEHY & TANK & FACILITY & 16000 & 672000 & 15624 & $18 G / 315 / 24 E$ \\
\hline 4091627 & 986 DEHY & TANK & FACILITY & 16000 & 672000 & 15633 & $186 / 315 / 24 E$ \\
\hline 4091428 & IBC DEHY & TANK & FACILITY & 16000 & 672000 & 12258 & $186 / 315 / 24 E$ \\
\hline 4091429 & 18G DEHY & TANK & FACILITY & 16000 & 672000 & 11465 & $186 / 315 / 24 E$ \\
\hline 4091630 & 180 DEHY & TANK & FACIIITY & 16000 & 672000 & 11107 & $180 / 315 / 24 E$ \\
\hline 4091431 & 18G DEHY & rank & FACILIYY & 16000 & 672000 & 11112 & $186 / 315 / 24 E$ \\
\hline 4091432 & 18G DEHY & TAMK & FACILITY & 16000 & 672000 & 15625 & $186 / 315 / 24 E$ \\
\hline 4091433 & 18G DEHY & TANK & FACILITY & 16000 & 672000 & 15634 & $186 / 315 / 24 E$ \\
\hline 4091434 & 186 DEHY & TANK & FACILITY & 16000 & 672000 & 12259 & $18 G / 31 S / 24 E$ \\
\hline 4091435 & 186 DEHY & TANK & FACILITY & 16000 & 672000 & 11466 & $18 G / 31 S / 24 E$ \\
\hline 4091436 & 195 DEHY & TANK & FACILITY & 16000 & 672000 & 11108 & $18 G / 315 / 24 E$ \\
\hline 4091437 & $180 \mathrm{bt} H Y$ & TANK & FACILITY & 16000 & 672000 & $\$ 1111$ & $186 / 315 / 24 E$ \\
\hline 4091438 & PBG DEHY & TANK & FACILITY & 96000 & 672000 & 15626 & $18 G / 315 / 24 E$ \\
\hline 4091439 & 186 DEHY & TANK & FACILITY & 16000 & 672000 & 15635 & $18 G / 315 / 24 E$ \\
\hline 4091440 & 18S DEHY & TANK & FACILITY & 16000 & 672000 & 12260 & $186 / 315 / 24 E$ \\
\hline 6091441 & 186 DEHY & TANK & FACILITY & 16000 & 672000 & 11467 & $986 / 315 / 24 E$ \\
\hline 4091442 & 18G DEKY & TANK & FACILITY & 16000 & 672000 & 19109 & $186 / 315 / 24 E$ \\
\hline 4091443 & 18G DEHY & TANK & FACILITY & 16000 & 672000 & 19190 & $186 / 315 / 24 E$ \\
\hline 4091151 & XOR STEAM & TANK & FACILITY & 500 & 21000 & 16 & SOR/30S/23E \\
\hline 4001152 & 30 STEAM & TANK & FACILITY & 500 & 21000 & 27 & $30 R / 30 \mathrm{~S} / 23 \mathrm{E}$ \\
\hline 4001154 & 1-35R CARH & TANK & FACILITY & 1000 & 42000 & 12463 & $35 R / 305 / 23 E$ \\
\hline 4091546 & $1-35 R$ CARN & TANK & FACILITY & 35 & 1470 & 15325 & $35 R / 30 S / 23 E$ \\
\hline 4099155 & $18 G$ & TANK & FACILITY & 30000 & 1260000 & 52127 & $186 / 315 / 24 E$ \\
\hline 4091156 & $3-8 R$ & TANK & FACILITY & 500 & 21000 & 15695 & $8 R / 30 S / 23 E$ \\
\hline 4091444 & 3.88 & TANK & FACILITY & 5200 & 218400 & 1278 & $8 R / 30 S / 23 E$ \\
\hline 4091445 & $3 \cdot 8 R$ & TANK & FACILITY & 5200 & 218400 & 15696 & $8 R / 30 S / 23 E$ \\
\hline 4001547 & $3-8 R$ & TANK & FACILITY & 35 & 9470 & 12712 & $8 R / 30 S / 23 E$ \\
\hline 4091157 & $1-17 R$ & TANK & FACILITY & 500 & 21000 & 9243 & $17 R / 30 S / 23 E$ \\
\hline 4091446 & $1-17 R$ & TANK & FACILITY & 5200 & 218400 & 15694 & $1 \mathrm{rR} / 30 \mathrm{~S} / 23 \mathrm{E}$ \\
\hline 4001447 & $1-17 R$ & TAMK & FACILITY & 4500 & 189000 & 15693 & $17 R / 30 S / 23 E$ \\
\hline 4091158 & $2-17 R$ & TANK & FACILITY & 500 & 21000 & 15692 & $17 k / 30 S / 23 E$ \\
\hline 4091648 & $2-17 R$ & TANK & FACILITY & 3000 & 126000 & 15691 & $17 R / 305 / 23 E$ \\
\hline 4091449 & $2-17 R$ & TANK & FACILITY & 5200 & 218400 & 15690 & $17 R / 30 S / 23 E$ \\
\hline 4091159 & $3-26 R$ & TANK & FACILITY & 500 & 21000 & 15989 & $26 R / 30 S / 23 E$ \\
\hline 4091450 & $3-26 R$ & TANK & FACILITY & 2000 & 84000 & 15991 & $26 R / 305 / 23 E$ \\
\hline 4091651 & $3-26 R$ & TANK & FACIIITY & 2000 & 84000 & 15990 & $262 / 30 S / 23 E$ \\
\hline 6091548 & $3-26 R$ & TAMK & FACILITY & 100 & 4200 & 13230 & $26 R / 305 / 23 E$ \\
\hline 4091960 & $2-27 R$ & TANK & FACILITY & 500 & 21000 & 16124 & $27 \pi / 30 S / 23 E$ \\
\hline 4091452 & $2-27 R$ & TANK. & FACILITY & 2000 & 84000 & 16125 & $27 R / 30 S / 23 E$ \\
\hline 4091453 & $2-27 R$ & TAMK & FACILITY & 2000 & 84000 & 16126 & $27 \mathrm{R} / 30 \mathrm{~S} / 23 \mathrm{E}$ \\
\hline 4091549 & $2 \cdot 27 R$ & TANK & FACILITYY & 100 & 4200 & 16142 & $27 R / 30 S / 23 E$ \\
\hline
\end{tabular}


1. Kern County Air Pollution Contol District

MPR-1 Permits to Operate - 1989

\begin{tabular}{|c|c|c|c|c|c|c|c|}
\hline $\begin{array}{l}\text { PERMIT } \\
\text { MUMBER } \\
\text { MEx=mEEIF= }\end{array}$ & $==2 x=2 x=1=\pi=$ & $\begin{array}{l}\text { OPER } \\
\text { DESC } \\
x===\end{array}$ & $\begin{array}{l}\text { ZATIONAL } \\
\text { CRIPTION } \\
==z==x==\end{array}$ & $\begin{array}{c}\text { TANK SIZE } \\
\text { (BBL) } \\
=x==x=x=x=\pi x\end{array}$ & $\begin{array}{c}\text { RATING } \\
\text { (GAL/BTU/HP) } \\
=========x=2=2=\end{array}$ & 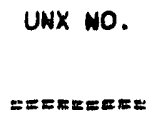 & 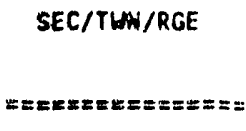 \\
\hline 4091161 & $2-35 R$ & TANK & FACILITY & 500 & 21000 & 16261 & $35 R / 30 S / 23 E$ \\
\hline 4091454 & $2-35 R$ & TANK & FACILITY & 2000 & 84000 & 14259 & $35 R / 30 S / 23 E$ \\
\hline 4091455 & $2 \cdot 35 R$ & TANK & FACILITY & 2000 & 84000 & 14260 & $35 R / 30 S / 23 E$ \\
\hline 4091550 & $2-35 R$ & TANK & FACILITY & 100 & 4200 & 14290 & $35 R / 30 S / 23 E$ \\
\hline 4091162 & $6.35 R$ & TANK & PACILITY & 1000 & 62000 & 18072 & $35 R / 30 S / 23 E$ \\
\hline 4091456 & $4-35 R$ & TANK & FACILITY & 2000 & 84000 & 18073 & $35 R / 305 / 23 E$ \\
\hline 4091551 & $4-35 R$ & TANK & FACILITY & 100 & 4200 & 18074 & $35 R / 30 S / 23 E$ \\
\hline 4091163 & 25S DEHY & TANK & FACILITY & 31000 & 1302000 & 371 & $25 s / 30 s / 24 E$ \\
\hline 4091457 & 25S DEHY & TANK & FACILPYY & 31000 & 1302000 & 372 & $255 / 305 / 24 E$ \\
\hline 4091458 & $25 S$ DEHY & TANK & FACILITY & 20500 & 861000 & 373 & $25 S / 30 S / 24 E$ \\
\hline 4091459 & $25 S$ DEHY & TANK & PACILITY & 20500 & 861000 & 374 & $25 S / 305 / 24 E$ \\
\hline 4091460 & 25S DEHY & TANK & FACILITY & 20500 & 861000 & 375 & $25 S / 30 S / 24 E$ \\
\hline 4091552 & 25S DEHY & TANK & FACILITY & 35 & 1470 & 16227 & $25 S / 30 S / 24 E$ \\
\hline 4091560 & 25S DEHY & TANK & FACILITY & 10250 & 430500 & 1294 & $25 S / 30 S / 24 E$ \\
\hline 4091561 & 25S DEHY & TANK & FACILITY & 5000 & 210000 & 1295 & $255 / 30 S / 24 E$ \\
\hline 4091562 & 25S DEHY & TANK & FACILITY & 1000 & 42000 & 51293 & $25 S / 30 S / 2.4 E$ \\
\hline 4091563 & 25S DEHY & TAMK & FACILITY & 200 & 8400 & 1292 & $25 S / 30 S / 24 E$ \\
\hline 4091564 & 255 DEHY & TANK & FACILITY & 200 & 8400 & 1293 & $258 / 30 S / 24 E$ \\
\hline 4091164 & 10G DEHY & TANK & FACILITY & 16000 & 672000 & 11103 & $106 / 315 / 24 E$ \\
\hline 4091461 & 10G DEHY & TANK & FACILITY & 16000 & 672000 & 11904 & $10 G / 31 S / 24 E$ \\
\hline 4091462 & 106 DEHY & IAMK & FACILITY & 16000 & 672000 & 11105 & $10 G / 315 / 24 E$ \\
\hline 4091565 & 10G DEHY & TANK & FACILITY & 3000 & 126000 & 51187 & $10 \mathrm{G} / 31 \mathrm{~S} / 26 \mathrm{E}$ \\
\hline 4091566 & 10G DEHY & TANK & FACILITY & 90 & 3780 & 11115 & $10 G / 315 / 24 E$ \\
\hline 4099567 & IOG DEHY & TANK & FACILITY & 12000 & 504000 & 11166 & $10 G / 31 S / 24 E$ \\
\hline 6091165 & 262 DEHY & TANK. & FACILITY & 2000 & 84000 & 12662 & $262 / 30 S / 22 E$ \\
\hline 4091463 & 262 DEHY & TANK & FACILITY & 1000 & 42000 & 12464 & $262 / 30 S / 22 E$ \\
\hline 4091464 & 262 DEHY & TANK & FACILITY & 1000 & 42000 & 12665 & $262 / 30 S / 22 E$ \\
\hline 4091568 & 262 DEHY & TANK & FACILITY & 1000 & 42000 & 1101 & $262 / 30 S / 22 E$ \\
\hline 4091166 & $4-29 R$ & TANK & FACILITY & 500 & 21000 & 1159 & $29 R / 30 S / 23 E$ \\
\hline 4091665 & $4-29 R$ & TANK & FACILITY & 500 & 21000 & 1156 & $29 R / 305 / 23 E$ \\
\hline 4091553 & $4-29 R$ & TANK & PACILITY & 35 & 1470 & 59427 & $29 R / 30 S / 23 E$ \\
\hline 4091167 & $2-33 k$ & TANK & FACILITY & 500 & 21000 & 1151 & $33 R / 30 S / 23 E$ \\
\hline 4091466 & $2 \cdot 33 R$ & TAMK & FACILITY & 500 & 21000 & 1150 & $33 R / 30 S / 23 E$ \\
\hline 4001554 & $2-33 R$ & TANK & FACILITY & 35 & 9470 & 51612 & $33 R / 30 S / 23 E$ \\
\hline 4091168 & $3-36 R$ & TANK & FACILITY & 500 & 21000 & 1160 & $34 R / 30 S / 23 E$ \\
\hline 4091467 & $3-34 R$ & TANK & FACILITY & 500 & 21000 & 1961 & $36 R / 30 S / 23 E$ \\
\hline 4091555 & $3-36 R$ & TANK & FACILITY & 35 & 1670 & 51695 & $34 R / 305 / 23 E$ \\
\hline 4091169 & $1-35 R$ & TANK & FACILITY & 500 & 21000 & 1884 & $35 R / 30 S / 23 E$ \\
\hline 4091468 & $9-35 R$ & TANK & FACILITY & 500 & 21000 & 1185 & $35 R / 305 / 23 E$ \\
\hline 4091556 & $8-35 R$ & TANK & FACIIITY & 35 & $16 m$ & $51 / 403$ & $350 / 30 S / 23 F$ \\
\hline 4081170 & $1 \cdot 36 R$ & TANK & EACILITY & 500 & 21000 & 1068 & $36 R / 305 / 23 E$ \\
\hline
\end{tabular}


1. Kern County Air Pollution Contol District

MPR-1 Permits to aperate 1989

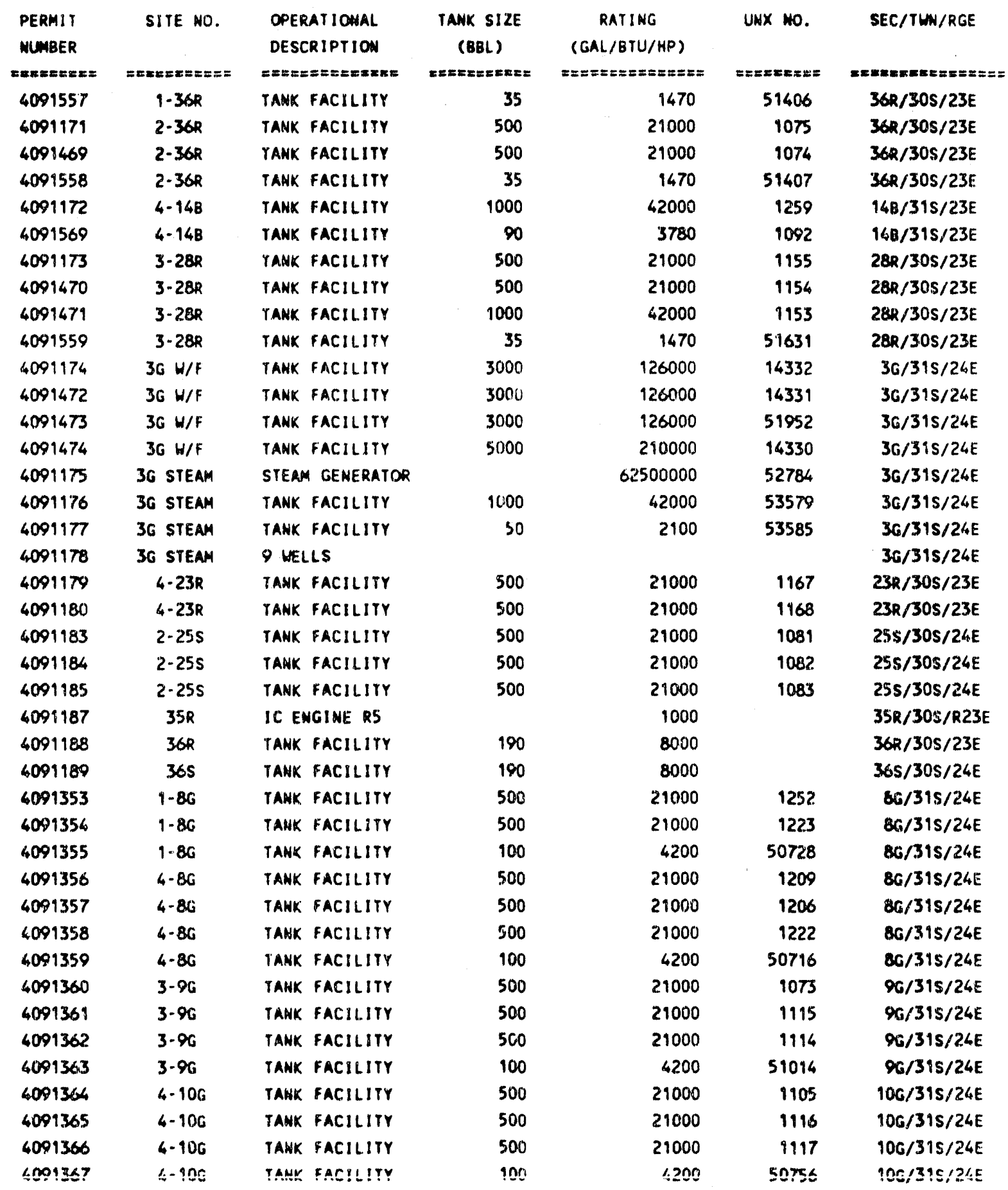




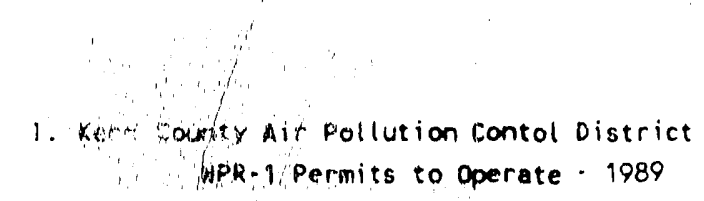

\begin{tabular}{|c|c|c|c|c|c|c|}
\hline $\begin{array}{l}\text { PERMIT } \\
\text { MUNBER } \\
x=m=x==\end{array}$ & $y \pi x=2 x=2 x==$ & $\begin{array}{l}\text { OPERATIONAL } \\
\text { DESCRIPTIION } \\
x==x=x=m=\pi x=x=\end{array}$ & $\begin{array}{l}\text { TANK SIZE } \\
(B B L) \\
======x=m=m=\end{array}$ & $\begin{array}{c}\text { RATING } \\
\text { (GAL/BTU/HP) } \\
=m==x=m===x=m=x\end{array}$ & $=2 \pi x=2 x=x$ & 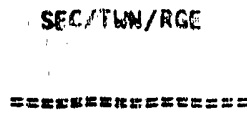 \\
\hline 4091368 & $3-116$ & TAMK FACILITY & 1000 & 42000 & 1195 & $116 / 315 / 24 E$ \\
\hline 4091369 & $3-116$ & TANK FACILITY & 1000 & 42000 & 1265 & $11 G / 315 / 24 E$ \\
\hline 4091370 & 3.116 & TANK FACILITY & 1000 & 42000 & 1245 & $116 / 395 / 24 E$ \\
\hline 4091371 & $3-116$ & TANK FACILITY & 100 & 4200 & 50786 & $116 / 315 / 24 E$ \\
\hline 4091372 & $1-120$ & TANK FACILITY & 500 & 21000 & 1110 & $12 G / 31 S / 24 E$ \\
\hline 4091373 & $1-12 G$ & TANK FACILITY & 35 & 1470 & 11223 & $126 / 315 / 24 E$ \\
\hline 4091374 & $1-12 G$ & TANK FACILITY & 35 & 1470 & 51628 & $12 G / 315 / 24 E$ \\
\hline 4091375 & $3-215$ & TAMK FACILITY & 500 & 21000 & 1129 & $215 / 30 S / 24 E$ \\
\hline 4091376 & $3 \cdot 215$ & TANK FACILITY & 100 & 4200 & 51062 & $21 \$ / 30 S / 24 E$ \\
\hline 4091570 & $3-215$ & TANK FACILITY & 500 & 21000 & 12.67 & $215 / 30 S / 24 E$ \\
\hline 4091377 & $3-25 s$ & TANK FACILITY & 500 & 21000 & 1121 & $25 S / 30 S / 24 E$ \\
\hline 4091378 & $3-25 s$ & TANK FACILITY & 500 & 21000 & 1122 & $25 S / 30 S / 24 E$ \\
\hline 4091379 & $3-25 S$ & TANK FACILIYY & 100 & 4200 & 59074 & $25 \$ / 30 S / 24 E$ \\
\hline 4091380 & $4-28 S$ & TANK FACILITY & 500 & 21000 & 1061 & $285 / 30 S / 24 E$ \\
\hline 4091381 & $4-28 S$ & TAMK FACILITY & 500 & 21000 & 1264 & $28 S / 30 S / 24 E$ \\
\hline 4091382 & $4-28 S$ & TAMK FACILITY & 500 & 21000 & 1257 & $285 / 305 / 24 E$ \\
\hline 4091383 & $4-285$ & TAHK FACILITY & 100 & 4200 & 51026 & $285 / 30 S / 24 E$ \\
\hline 4091384 & $3-275$ & TANK FACILITY & 1000 & 42000 & 1228 & $275 / 30 S / 24 E$ \\
\hline 4091385 & $3-27 s$ & TANK FACILITY & 1000 & 42000 & 1256 & $27 S / 30 S / 24 E$ \\
\hline 4091386 & $3-275$ & TANK FACILITY & 100 & 4200 & 51038 & $27 S / 30 S / 24 E$ \\
\hline 4091387 & $4-26 s$ & TAHK FACILITY & 500 & 21000 & 1204 & $265 / 305 / 24 E$ \\
\hline 4091388 & $4-265$ & TANK FACILITY & 500 & 21000 & 1120 & $265 / 30 S / 24 E$ \\
\hline 4091389 & $4-26 s$ & TAMK FACILITY & 500 & 21000 & 1227 & $265 / 30 S / 24 E$ \\
\hline 4091390 & $4-265$ & TANK FACILITY & 100 & 4200 & 51050 & $265 / 30 S / 24 E$ \\
\hline 4081391 & $4-365$ & TANK FACILSTY & 500 & 21000 & 1128 & $36 S / 30 S / 24 E$ \\
\hline 4091392 & 4.365 & TANK FACILITY & 500 & 21000 & 1942 & $365 / 30 S / 24 E$ \\
\hline 4091393 & $4 \cdot 365$ & TANK FACILITY & 100 & 4200 & $500 \mathrm{red}$ & $365 / 305 / 24 E$ \\
\hline 4091394 & $3-66$ & TANK FACILITY & 500 & 21000 & $126 \%$ & $66 / 315 / 24 E$. \\
\hline $40913 \% 5$ & 3.66 & TAHK FACILITY & 500 & 21000 & 1254 & $6 G / 315 / 24 E$ \\
\hline 4091396 & $3-60$ & TAMK EACILITY & 250 & 10500 & 2045 & 6G/3IS/24E \\
\hline 4091397 & $3-66$ & TANK FACILITY & 100 & 4200 & 50798 & $6 G / 315 / 24 E$ \\
\hline 4091578 & $29 R$ & SAFETY FLARE & & 44580000 & & $29 / 30 S / 23 E$ \\
\hline 4091579 & 299 & SAFETY FLARE & & 44580000 & & $29 / 30 S / 2 \mathrm{JE}$ \\
\hline 4091580 & 298 & SAFETY FLARE & & 28000 & & $29 / 30 S / 23 E$ \\
\hline 4091582 & $35 R$ & CATERPILLAR K67 & & 490 & 54302 & $35 / 30 S / 23 E$ \\
\hline 4091583 & $35 R$ & CATTERPILLAR KOG & & 490 & 54299 & $35 / 30 S / 23 E$ \\
\hline 4091584 & $35 k$ & CATERPILLAR K69 & & 690 & 54318 & $35 / 30 S / 23 E$ \\
\hline 4091585 & $180 \mathrm{~W} W$ & WAUK. F2475CU & & 430 & 12789 & $18 / 315 / 24 E$ \\
\hline 4091586 & $3 G W / F P 3$ & WAUK. H24T5 & & 430 & 15469 & $3 / 315 / 24 E$ \\
\hline 4091587 & $3 G W / F \quad P 2$ & WAUK. H2475 & & 430 & 13296 & $3 / 315 / 24 E$ \\
\hline $4 \bar{v} \overline{y i} \overline{0}$ & $\overline{3} 6 w / r k i$ & HAUK. MLUTT & & ¿50 & & SiJisizuE \\
\hline
\end{tabular}


1. Kern County Air Pollution Contol District NPR-1 Permits to Operate. 1089

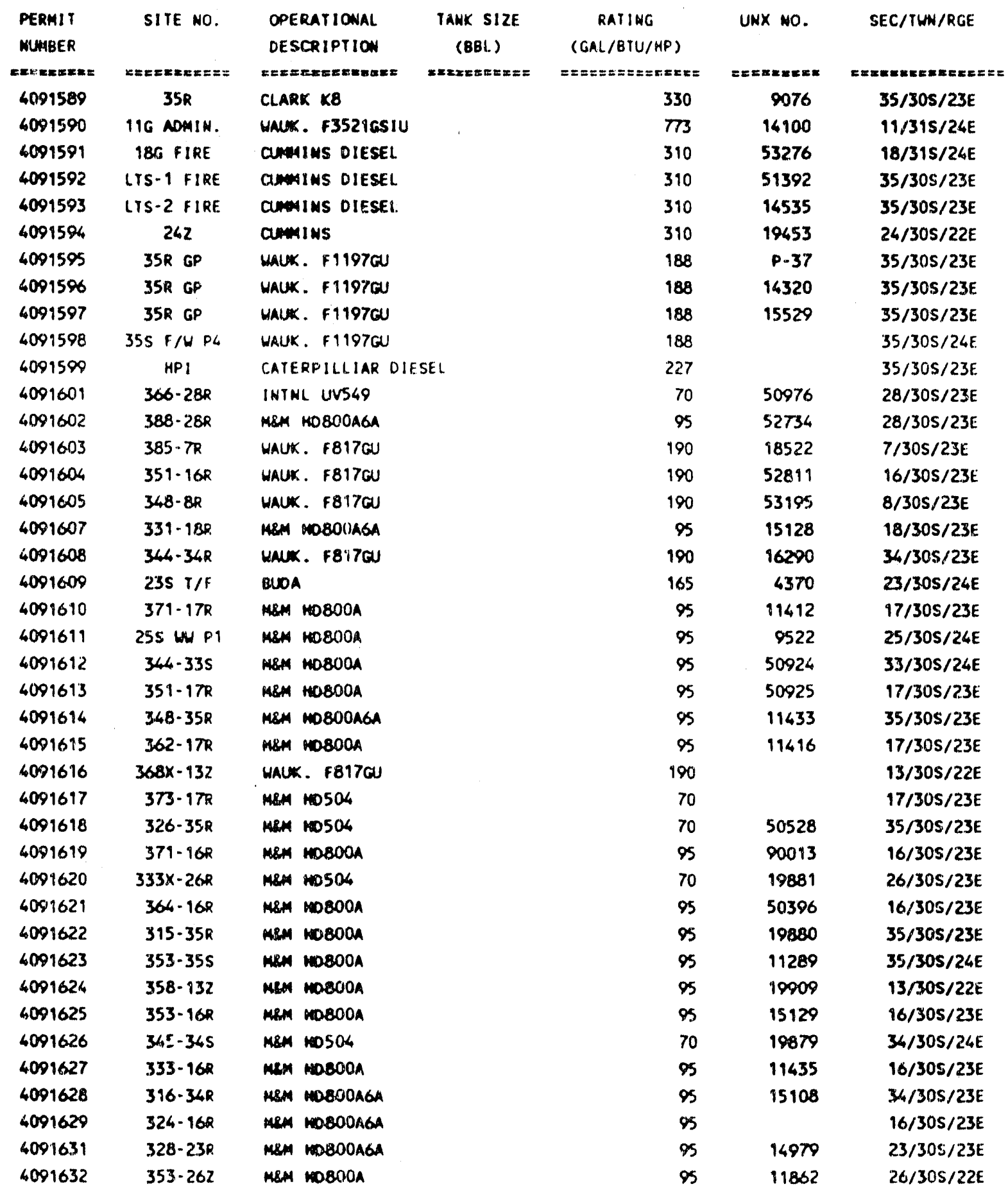


Kern County Air Pollution Contol Distict

NPR-1 Permits to Operote - 1989

\begin{tabular}{|c|c|c|c|c|c|c|}
\hline $\begin{array}{l}\text { PERMIT } \\
\text { MUABER } \\
M=E=E x=E=K\end{array}$ & 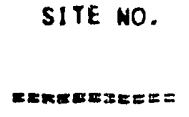 & $\begin{array}{l}\text { OPERATIONAL } \\
\text { DESCRIPTION } \\
x=x=x=x=m=x==x\end{array}$ & $\begin{array}{l}\text { TAMK SIZE } \\
(8 B L) \\
==x=x=x=x=m\end{array}$ & $\begin{array}{c}\text { RAIIHG } \\
\text { (GAL/BTU/HP) } \\
=\pi===z=\pi==\pi=z=\end{array}$ & UNX NO. & SEC/TLW/RGE \\
\hline 4081633 & $355-15 R$ & MEM HOSOOA & & 95 & 12630 & $15 / 30 \mathrm{~S} / 23 \mathrm{E}$ \\
\hline 4091634 & $328-91$ & MEN MDBOOAGA & & 95 & 50397 & $9 / 30 S / 23 E$ \\
\hline 4091635 & $3684-325$ & MEM HDSOOA & & 95 & $503 \infty$ & $32 / 305 / 24 E$ \\
\hline 4091636 & $361-262$ & $\operatorname{mos} 04$ & & 70 & 19012 & $26 / 30 S / 22 E$ \\
\hline 4091637 & $35 S$ F/U P5 & MBM MDBOOAGA & & 95 & 11411 & $35 / 30 S / 24 E$ \\
\hline 4091638 & $318-32 s$ & MEA MOBOOA & & 95 & 19521 & $32 / 30 S / 24 E$ \\
\hline 4091639 & $354-242$ & MEM MOBOOAGA & & 95 & 14980 & $24 / 30 S / 22 E$ \\
\hline 4091640 & $336-36 R$ & WAUK. VRCBIOU & & 88 & 13082 & $34 / 30 S / 23 E$ \\
\hline 4091641 & $317-15 R$ & MEM 1OOBOOA & & 95 & 15131 & $15 / 30 S / 23 E$ \\
\hline 4091642 & $363-262$ & WAUK. FB17GU & & 190 & 52810 & $26 / 30 S / 22 E$ \\
\hline 4091643 & 255 UWS & MEM MDBOOA & & 95 & 11419 & $25 / 30 S / 24 E$ \\
\hline 4091644 & $357-8 R$ & MOBOAOAGA & & 95 & & $8 / 30 S / 23 E$ \\
\hline 4091645 & 255 WN P3 & MEM HOBOOAGA & & 95 & 50901 & $25 / 30 S / 2.4 E$ \\
\hline 4091646 & $352 \cdot 262$ & HSA NOBOOA & & 95 & 11869 & $26 / 30 S / 22 E$ \\
\hline 4091647 & $342-17 R$ & MEM HOBODAGA & & 95 & 11312 & $17 / 30 S / 23 E$ \\
\hline 4091648 & $374-262$ & Mem MOSOL & & 70 & 19913 & $26 / 30 S / 22 \mathrm{E}$ \\
\hline 4091649 & $373-16 R$ & Men nosoon & & 95 & 11410 & $16 / 30 S / 23 E$ \\
\hline 4091650 & $2 \cdot 36 \mathrm{~S} / \mathrm{P}$ & WAUK. VRG3100 & & 88 & 15565 & $36 / 30 S / 23 E$ \\
\hline 4091651 & $333-15 R$ & MRA HOSOOAGA & & 95 & 16295 & $15 / 305 / 23 E$ \\
\hline 1091652 & $584-262$ & MEH NOBOOA & & 95 & 50929 & $26 / 30 S / 22 E$ \\
\hline 4091653 & $362-18 R$ & MEM HDBOOAGA & & 95 & 11290 & $18 / 30 S / 2.3 E$ \\
\hline 4081654 & $318-27 R$ & MRM NOBODAGA & & 95 & & $27 / 30 \mathrm{~S} / 23 \mathrm{E}$ \\
\hline 4091655 & $378-22 R$ & MEA MOBOOA & & 95 & 11425 & $22 / 30 S / 23 E$ \\
\hline 4091656 & $371 X-32 R$ & MEM MOBOCAGA & & 95 & 50926 & $32 / 30 S / 23 E$ \\
\hline 6091657 & $373-15 R$ & MOAOAOA & & 95 & 11434 & $15 / 30 S / 23 E$ \\
\hline 4091658 & $313-34 S$ & Men NOSOL & & 70 & 19911 & $34 / 30 S / 24 E$ \\
\hline 4091659 & $335-15 R$ & MEH Mnsoon & & 95 & 15106 & $15 / 30 S / 23 E$ \\
\hline 4091660 & $337-88$ & MeM MDSOOA & & 95 & 50398 & $8 / 30 S / 23 E$ \\
\hline 4091661 & $385-242$ & MEM HOBOOA & & 95 & 11421 & $24 / 30 S / 22 E$ \\
\hline 4091662 & $34,5-288$ & MEA MOSOOAGA & & 95 & 19763 & $28 / 30 S / 23 E$ \\
\hline 4091663 & 255 wS & MRM DSOOAGA & & 95 & 19436 & $25 / 30 S / 24 E$ \\
\hline 4091664 & $326-8 R$ & men 10800464 & & 85 & 11287 & $8 / 30 S / 23 E$ \\
\hline 4091665 & $313 \cdot 17 R$ & MEM MDSOOA & & 85 & 50539 & $17 / 30 \mathrm{~S} / 23 \mathrm{E}$ \\
\hline 40016063 & $368-28 R$ & MEA MOS00A6A & & 95 & 11423 & $28 / 30 S / 23 E$ \\
\hline 4091667 & $3184-18 R$ & MEA MOSOOAGA & & 95 & 50400 & $18 / 30 S / 23 E$ \\
\hline 4091668 & $377-15 R$ & Men MOEDOAGA & & $\%$ & & $15 / 30 S / 23 E$ \\
\hline 4091669 & $315-82$ & nem mosoon & & 95 & & $8 / 30 S / 23 E$ \\
\hline 4091670 & $394-242$ & mim nosocansa & & 95 & 52735 & $24 / 30 S / 22 E$ \\
\hline 4001671 & $367-7 R$ & MEA MODOOA & & 95 & 50928 & $7 / 30 S / 23 E$ \\
\hline 6001672 & $388-82$ & HALK. FB17GU & & 190 & 12426 & $8 / 30 S / 23 E$ \\
\hline Loviotión & $30.7-\pi$ & 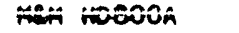 & & 85 & $15 i 07$ & Tizosiaje \\
\hline
\end{tabular}


1. Kern County Air Pollution Contol District

NPR-1 Permizs to Operate - 1989

\begin{tabular}{|c|c|c|c|c|c|c|}
\hline $\begin{array}{l}\text { PERMIT } \\
\text { MUMBER } \\
=x=x=x=2=\end{array}$ & 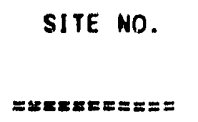 & $\begin{array}{l}\text { OPERATIONAL } \\
\text { DESCRIPYION } \\
====x=x==\pi=2 k=\end{array}$ & $\begin{array}{c}\text { TANK SIZE } \\
\text { (BBL) } \\
===8=5=8=5=\end{array}$ & $\begin{array}{c}\text { RATING } \\
(\text { GAL/BTU/HP) } \\
====== \pm===z==z\end{array}$ & UNX NO. & 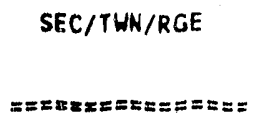 \\
\hline 4091675 & $356-34 \mathrm{~S}$ & MeM NDBOOAGA & & 95 & & $34 / 305 / 24 \mathrm{E}$ \\
\hline 4091676 & 35s F/W P6 & MeM Ho800A6A & & 95 & 11418 & $35 / 30 S / 246 E$ \\
\hline 4091677 & $374-335$ & MEM NO504 & & 70 & 19876 & $33 / 30 S / 24 \mathrm{E}$ \\
\hline 4091678 & $336-7 R$ & Men HO800A & & 95 & 11284 & $7 / 30 S / 23 E$ \\
\hline 4091679 & $1-242 \mathrm{~S} / \mathrm{P}$ & HAUK. VRG31OU & & 88 & 16391 & $24 / 30 s / 22 E$ \\
\hline 4091680 & $384-33 s$ & WAUK. VRG310U & & 88 & 16323 & $33 / 30 S / 24 E$ \\
\hline 4091681 & $315-16 R$ & WAUK. VRG310U & & 88 & 15568 & $16 / 30 S / 23 E$ \\
\hline 4091683 & $35 R \mathrm{GP}$ & WAUK. VRG310U & & 88 & & $35 / 30 S / 23 E$ \\
\hline 4091684 & $3 \pi-15 R$ & WAUK. VRG31OU & & 88 & 16387 & $15 / 30 S / 23 E$ \\
\hline 4091685 & $35 R$ GP & WAUK. VRG310U & & $8 B$ & & $35 / 30 S / 23 E$ \\
\hline 4091686 & $353 \cdot 15 R$ & WAUK. VRG310U & & 88 & 16390 & $15 / 30 \mathrm{~S} / 23 \mathrm{E}$ \\
\hline 4091687 & $333 A-34 S$ & WAUK. VRG31OU & & 88 & 15547 & $34 / 30 S / 24 E$ \\
\hline 4091688 & $348-30 s$ & WAUK. VRG310U & & 88 & 15546 & $30 / 30 S / 24 E$ \\
\hline 4091690 & $313-15 R$ & INTNL UVS49 & & 70 & 50966 & $15 / 30 S / 23 E$ \\
\hline 4091691 & $1-315 S / P 4$ & HAUK. VRG310U & & 88 & 13147 & $31 / 30 S / 24 E$ \\
\hline 4091692 & $316-23 R$ & HAUK. VRG310U & & 88 & 15554 & $23 / 30 S / 23 E$ \\
\hline 4091694 & $373-335$ & WAUK. VRG310U & & 88 & 17108 & $33 / 30 S / 24 E$ \\
\hline 4091695 & $361-315$ & HALK. VRG310U & & 88 & 16388 & $31 / 30 S / 24 E$ \\
\hline 4091696 & $384 \cdot 17 R$ & HAUK. VRG31OU & & 88 & 13033 & $17 / 30 \mathrm{~S} / 23 \mathrm{E}$ \\
\hline 4091697 & $346-345$ & MEHA HO80OAGA & & 95 & 50923 & $34 / 30 S / 24 E$ \\
\hline 4091698 & $322-16 R$ & HAUK. VRG310U & & 88 & & $16 / 30 S / 23 E$ \\
\hline 4091699 & $355 \cdot 142$ & WAUK. VRG3YOU & & 88 & 15568 & $14 / 30 S / 22 E$ \\
\hline 4091700 & $35 R$ GP & WAUK. VRG310U & & 88 & 15549 & $35 / 30 S / 23 E$ \\
\hline 4091701 & $315-14 R$ & WAUK. VRGB1OU & & 88 & 14101 & $14 / 30 S / 23 E$ \\
\hline 4091702 & 35R GP & WAUK. VRG310U & & 88 & 13985 & $35 / 30 S / 23 E$ \\
\hline 4091703 & $35 \mathrm{~S} / \mathrm{H} P 7$ & WAUK. F1197GU & & 188 & 14321 & $35 / 305 / 24 E$ \\
\hline 4091704 & $364-32 s$ & HAUK. VRG31QU & & 88 & 15550 & $32 / 30 S / 24 E$ \\
\hline 4091706 & $377 \cdot 29 R$ & HAUK. VRGS10U & & 88 & 12872 & $2.9 / 30 S / 23 E$ \\
\hline 4091707 & $35 R G P$ & WAUK. F3521GSIU & & $i 73$ & 14160 & $35 / 30 S / 23 E$ \\
\hline 4091708 & $352-32 s$ & WAUK. VRG310U & & 88 & 15570 & $32 / 30 S / 24 E$ \\
\hline 4091709 & $324-33 s$ & WALK. VRG310U & & 88 & 15548 & $33-30 S / 24 E$ \\
\hline 4091710 & $383-36 s$ & WAUK. VRG310U & & 88 & 15557 & $36 / 30 \mathrm{~S} / 24 \mathrm{E}$ \\
\hline 4099711 & $353 \% 97 R$ & WAUK. VRG31OU & & 88 & 15555 & $17 / 30 \mathrm{~S} / 23 \mathrm{E}$ \\
\hline 4091712 & $333-35 s$ & WAUK. VRG310U & & 88 & 15556 & $35 / 30 S / 24 E$ \\
\hline 4001713 & 35R GP FIRE & CUMINS DIESEL & & 310 & 12052 & $35 / 30 S / 23 E$ \\
\hline 4091714 & $323-345$ & MAUK. VRG310U & & 88 & 15564 & $3 / 4 / 30 \mathrm{~S} / 24 \mathrm{E}$ \\
\hline 4091715 & $337-15 R$ & MEM HOSOL & & 70 & 50584 & $15 / 30 \mathrm{~S} / 23 \mathrm{E}$ \\
\hline 4001716 & $376.23 R$ & WAUK. VRG31OU & & 88 & 15571 & $23 / 30 S / 23 E$ \\
\hline 4001797 & $315-15 R$ & MOA & & 70 & 19875 & $15 / 30 \mathrm{~s} / 23 \mathrm{~F}$ \\
\hline 6091718 & 35R GP & INTHL UVS49 & & 70 & 12643 & $35 / 30 \mathrm{~S} / 23 \mathrm{E}$ \\
\hline 4099719 & $377-8 R$ & MBM HDSOL & & 70 & 19877 & $8 / 30 S / 23 E$ \\
\hline
\end{tabular}


1. Kern County Air Pollution Contol District

NPR- 1 Permits to Operote 1989

\begin{tabular}{|c|c|c|c|c|c|c|}
\hline $\begin{array}{l}\text { PERM!T } \\
\text { NUMBER } \\
=\pi \approx=\pi= \pm=\pi\end{array}$ & $=x=\pi x=z=\pi n=$ & $\begin{array}{l}\text { OPERATIONAL } \\
\text { DESCRIPTION } \\
======\pi==\pi=\pi \mathrm{m} x\end{array}$ & $\begin{array}{l}\text { TANK SIZE } \\
\text { (BBL) } \\
==\pi=\pi=\pi=z=z\end{array}$ & $\begin{array}{c}\text { RATING } \\
(\text { GAL/BTU/HP) } \\
===== \pm===\pi==\pi=\end{array}$ & 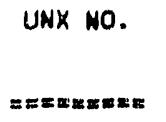 & 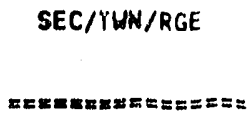 \\
\hline 4091720 & $374-66$ & MeM HOSO4 & & 70 & 19762 & $6 / 31 \$ / 24 E$ \\
\hline 4091721 & $343-345$ & INTNL US549 & & 70 & 50965 & $34 / 30 S / 24 E$ \\
\hline 4091722 & $342-28$ & MEM HOSOL & & 70 & 19914 & 2/31S/23E \\
\hline 4099723 & $368-8 R$ & MEA HOSOL & & 70 & 11413 & $8 / 305 / 23 E$ \\
\hline 4091724 & $2-29 R \mathrm{~s} / \mathrm{P}$ & INTNL UN549 & & 70 & 50968 & $29 / 30 S / 23 \mathrm{E}$ \\
\hline 4091725 & $325-34 S$ & WAUK. VRG3IOU & & 88 & 16386 & $34 / 305 / 24 E$ \\
\hline 4091726 & $364-262$ & WAUK. F817CU & & 190 & 52704 & $26 / 30 S / 22 E$ \\
\hline 4091727 & $367-22 R$ & WAUK. VRG265U & & 67 & 11935 & $22 / 30 S / 23 E$ \\
\hline 4091728 & $333-345$ & WAUK. VRGZ265U & & 67 & 11887 & $34 / 30 S / 24 E$ \\
\hline 4091729 & $382-17 R$ & HAUK. VRG265U & & 67 & 13092 & $17 / 30 S / 23 E$ \\
\hline 4091730 & $65-20$ & HAUK. VRG265U & & 67 & & $2 / 315 / 24 E$ \\
\hline 4091731 & $311-16 R$ & HAUK. VRG265U & & 67 & & 16/30S/23E \\
\hline 4091732 & $42-3.3 R$ & WAUK. VRG265U & & 67 & 13007 & $33 / 30 S / 23 E$ \\
\hline 4091733 & $353-345$ & WAUK. VRG265U & & 67 & & $3 / 4 / 305 / 24 \mathrm{E}$ \\
\hline 4091734 & $35-116$ & WAUK. VRG265U & & 67 & 12897 & $11 / 315 / 24 \mathrm{E}$ \\
\hline 4091735 & $2-25 \mathrm{~s} s / \mathrm{P}$ & WAUK. VRG265U & & 67 & 12899 & $25 / 30 S / 24 E$ \\
\hline 4091736 & $314-345$ & WAUK. VRG265U & & 67 & & $34 / 30 S / 24 E$ \\
\hline 4091739 & $324 \cdot 325$ & WAUK. VRG265U & & 67 & & $32 / 30 S / 24 E$ \\
\hline 4091740 & $304-262$ & WAUK. VRG265U & & 67 & & $26 / 30 S / 22 E$ \\
\hline 4091741 & $87-5 G$ & WAUK. VRG265U & & 67 & 13093 & $5 / 315 / 24 E$ \\
\hline 4091742 & $331-16 R$ & WAUK. FB17GU & & 190 & 53116 & $16 / 30 S / 23 E$ \\
\hline 4091743 & $44 A-36 R$ & WAUK. VRG265U & & 67 & 13091 & $36 / 30 S / 23 E$ \\
\hline 4091744 & $357 \cdot 35 R$ & HAUK. VRG265U & & 67 & 11878 & $35 / 30 S / 23 E$ \\
\hline 4091745 & LTS-1 & WAUK. 579200 & & 793 & 13397 & $35 / 30 S / 23 E$ \\
\hline 4091746 & $4-28 R \mathrm{~S} / \mathrm{P}$ & WAUK : :G265U & & 67 & 11925 & $28 / 30 S / 23 E$ \\
\hline 4091747 & $364 x-335$ & WAUK, VRG310U & & 88 & 11193 & $33 / 30 S / 24 E$ \\
\hline 4091748 & $354-262$ & WAUK. VRG265U & & 67 & 11940 & $26 / 30 S / 22 E$ \\
\hline 4091749 & $1.36 R \mathrm{~s} / \mathrm{P5}$ & WAUK. VRG265U & & 67 & 19359 & $36 / 30 S / 23 E$ \\
\hline 4091750 & $389-365$ & HAUK. VRG265U & & 67 & 11942 & $36 / 305 / 24 E$ \\
\hline 4091754 & $51-10$ & HAUK. VRG2.65U & & 67 & 11928 & $26 / 30 S / 22 E$ \\
\hline 4091755 & LTS -2 & WAUK. L57920DU & & 793 & 14772 & $35 / 30 S / 23 E$ \\
\hline 4091756 & $322-17 R$ & HOSOOA & & 95 & & $17 / 30 S / 23 E$ \\
\hline 4091738 & $35 R$ & CATERPILLAR $X G B$ & & 490 & 54313 & $35 / 305 / 23 E$ \\
\hline
\end{tabular}


11. Kern County Air Pollution Contol District NPR-1 Authorities to Construct. 1989

\begin{tabular}{|c|c|c|c|c|c|c|}
\hline $\begin{array}{l}\text { PERMIT } \\
\text { NUMBER } \\
==:=:==\approx=\end{array}$ & 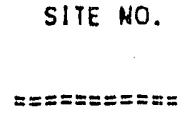 & $\begin{array}{l}\text { OPERATIONAL } \\
\text { DESCRIPTION } \\
==============\end{array}$ & $\begin{aligned} & \text { TANK SIZE }(B B L) \\
&(B L) \\
&=========0\end{aligned}$ & $\begin{array}{c}\text { RATING } \\
\text { (GAL/BTU/HP) } \\
====== \pm===\pi====\end{array}$ & $==x=z=x=$ & 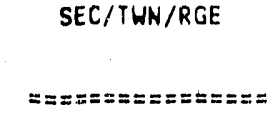 \\
\hline 4091197 & $27 R$ & TANK FACILITY & 950 & 39900 & & $27 R / 31 S / 23 E$ \\
\hline 4091198 & $35 R$ & TANK FACILITY & 500 & 21000 & & $35 / 30 S / 23 E$ \\
\hline 4091199 & $35 R$ & TANK FACILITY & 500 & 21000 & & $35 / 30 \mathrm{~S} / 23 \mathrm{E}$ \\
\hline 4091077 & $8 R$ & ELECT COMP K62 & & 1750 & 54685 & $8 / 30 \mathrm{~S} / 23 \mathrm{E}$ \\
\hline 4091077 & $8 R$ & ELECT COAP K61 & & 1750 & 54667 & $8 / 30 S / 23 E$ \\
\hline 4091078 & $8 R$ & GLYCOL SYSTEM & & 0.585 & & $8 / 30 \mathrm{~S} / 23 E$ \\
\hline 4091079 & 335 & ELECT COMP K65 & & 1750 & & $33 / 30 S / 24 E$ \\
\hline 4091079 & 335 & ELECT COMP KS3 & & 1750 & & $33 / 30 \mathrm{~S} / 24 \mathrm{E}$ \\
\hline 4091079 & 335 & ELECT COMP K64 & & 1750 & & $33 / 30 \mathrm{~S} / 24 \mathrm{E}$ \\
\hline 4091080 & 335 & GLYCOL SYSTEM & & 1.32 & & $33 / 30 S / 24 E$ \\
\hline $4091081 A$ & $30 R$ & IC ENGINE K71 & & 1500 & 400185 & $30 / 30 S / 23 E$ \\
\hline $4091082 A$ & $30 R$ & IC ENGINE KT2 & & 1500 & 400184 & $30 / 30 S / 23 E$ \\
\hline 4091181 & $23 \mathrm{~S}$ & TANK FACILITY & 40000 & 1680000 & 1283 & $23 / 30 S / 24 E$ \\
\hline 4091182 & $23 \mathrm{~s}$ & TANK FACILITY & 40000 & 1680000 & 1284 & $23 / 30 S / 24 E$ \\
\hline 4091190 & $32 R$ & TAAK FACILITY & 400 & 16800 & & $32 / 30 \mathrm{~S} / 23 \mathrm{~F}$ \\
\hline 4091571 & $3 n e$ & TANK FACILITY & 1000 & 42000 & 12463 & $30 / 30 S / 23 E$ \\
\hline 4081572 & swo & TANK FACILITY & 210 & 8820 & 53171 & $30 / 30 s / 23 E$ \\
\hline 4091573 & $30 R$ & TANK FACILITY & 210 & 8820 & 53172 & $30 / 30 S / 23 E$ \\
\hline 4091574 & 30R & TANK FACILITY & 250 & 10500 & 1234 & $30 / 30 \mathrm{~S} / 23 \mathrm{E}$ \\
\hline 4091575 & $30 R$ & TANK FACILITY & 35 & 1470 & 19330 & $30 / 30 S / 23 E$ \\
\hline 4091576 & $30 R$ & TANK FACILITY & 35 & 1470 & N/A & $30 / 30 S / 23 E$ \\
\hline 4091577 & 267. & TANK FACILITY & 100 & 4200 & & $26 / 30 \mathrm{~S} / 22 \mathrm{E}$ \\
\hline $4091175 A$ & 3G STEAM & STEAM GEHERATOR & & 62500000 & 52784 & $3 G / 31 S / 24 E$ \\
\hline 4091176 A & 3G STEAM & TANK FACILITY & 1000 & 42000 & 53579 & $36 / 31 S / 24 E$ \\
\hline $4091177 \AA$ & 3G STEAM & TANK FACILITY & 150 & 2100 & 53585 & $36 / 31 S / 24 E$ \\
\hline $4091178 \AA$ & $3 G$ STEAM & 9 WELLS & & & & $3 G / 31 S / 24 E$ \\
\hline $4091192 \mathrm{~A}$ & 3G STEAM & STEAM GENERATOR & & 62500000 & 52784 & $3 G / 31 S / 24 E$ \\
\hline $4091193 \mathrm{~A}$ & 3G STEAM & STEAM GENERATOR & & 62500000 & 52784 & $3 G / 31 S / 24 E$ \\
\hline $4091184 A$ & $3 G$ STEAM & STEAM GEHERATOR & & 62500000 & 52784 & $3 G / 31 S / 24 E$ \\
\hline $4091195 \mathrm{~A}$ & 3G STEAM & STEAM GENERAYOR & & 62500000 & 52784 & $3 G / 315 / 24 E$ \\
\hline 4091196 A & 3G STEAM & STEAYI GENERATOR & & 62500000 & 52784 & $3 G / 31 S / 24 E$ \\
\hline $4091136 \mathrm{E}$ & $1 \cdot 7 R$ & TANK FACILITY & 2000 & 84000 & 50296 & $7 R / 30 S / 23 E$ \\
\hline 4091401 & $1-7 R$ & TANK FACILITY & 2000 & 84000 & 50297 & $7 R / 30 S / 23 E$ \\
\hline 4091402 & $1-7 R$ & TAI'K FACILITY & 750 & 31500 & 50295 & $7 R / 30 S / 23 E$ \\
\hline 4091531 & $1-7 R$ & TANK FACILITY & 500 & 21000 & 15623 & $7 R / 30 S / 23 E$ \\
\hline 4091181 & 235 & TANK FACILITY & 40000 & 1680000 & 1283 & $23 S / 30 S / 24 E$ \\
\hline 4091182 & 235 & TANK FACILITY & 40000 & 1680000 & 1284 & $235 / 30 S / 24 E$ \\
\hline 4091190 & 325 & TANK FACILIYI & 400 & 16800 & & $32 R / 30 S / 23 E$ \\
\hline 4091581 & $18 G$ & TANK FACILITY & 154 & 6468 & & $18 G / 315 / 24 E$ \\
\hline
\end{tabular}




\section{Kern County Air Pollution Control District Authorities to Construct - 1988}

A/C No.

o 4091081-082

() 4091577

o 4091017B-020B,023B-026B, $030 \mathrm{~B}, 034 \mathrm{~B}, 036 \mathrm{~B}, 037 \mathrm{~B}$, 050B-052B,057B-070B

o

4091038B,071B,072B

o $4094049 \mathrm{~A}$

o $\quad 4091181$

() $\quad 4091182$

() $\quad 4091077$

() $\quad 4091078$

o) 4094079

○ $\quad 4091080$
Description

o Installation of two $1000 \mathrm{HP}$ gas-fired piston engines at 2-30R

o $\quad 26 Z$ Slop oil tank

- Modify permits to operate 31 existing gas-fired internal combustion engines to establish appropriate emission sampling limits for six criteria pollutants and to establish emission rate changes for $\mathrm{NOx}$ and $\mathrm{CO}$

- Modify permits to operate 3 existing gas-fired internal combustion engines to establish appropriate emission sampling limits for six criteria pollutants and to establish emission rate changes for $\mathrm{NO} x$ and $\mathrm{CO}$

- Modify permits to operate 1 Delavel compressor $\mathrm{K} 10$ to establish appropriate emission sampling limits for six criteria pollutants and to establish emission rate changes for $\mathrm{NOx}$ and $\mathrm{CO}$

o One 40,000 bbl Tank (UNX1283@ 23S)

o One 40,000 bbl Tank (UNX1284@ 23S)

- $\quad$ 8R Two Electric compressors

o $\quad$ 9R Glycol Regeneration System

o $\quad 33 \mathrm{~S}$ Three Electric Compressors

o $\quad 33 S$ Glycol Regeneration System 


\section{U. S. Fish and Wildlife Service \\ Permits and Biological Opinions}

\section{PERMITS}

1. Fish and Wildlife Handling Permit (Permit PRT-683011).

- Issued from the Fish and Wildlife Service to EG\&G Energy Measurements, Inc, effective 12/22/90; expires $8 / 31 / 90$.

- Authorizes handling of the San Joaquin kit fox, blunt-nosed leopard lizard, giant kangaroo rat, and Tipton kangaroo rat.

- Authorized capture and relocation of kit foxes to Naval Petroleum Reserve No. 1 .

\section{BIOLOGICAL OPINIONS}

1. Formal endangered species consultation on the petroleum development program at maximum efficient rate at NPR-1.

- Opinion rendered in response to a request for consultation from the U.S. Department of Energy.

- Opinion concluded that petroleum development at NPR-1 under maximum efficient rate of extraction program is not likely to jeopardize the continued existence of the San Joaquin kit fox, blunt-nosed leopard lizard, or giant kangaroo rat.

- Issued 12/16/87; terms of incidental take through FY89.

2. Formal endangered species consultation concerning the construction and operation by Texaco, Inc. of a four-mile crude oil pipeline to the $25 \mathrm{~S}$ lease automatic custody transfer on the Elk Hills Naval Petroleum Reserve, Kern County, California.

- Opinion rendered in response to a request for consultation from the U.S. Department of Energy.

- Opinion concluded that "proposed construction, maintenance, and operation of the crude oil pipeline is not likely to jeopardize the continued existence of the San Joaquin kit fox, blunt-nosed leopard lizard, giant kangaroo rat or Tipton kangaroo rat. Critical habitat has not been determined for these species; therefore, none will be destroyed or adversely modified." 
3. Formal endangered species consultation for the construction and operation by Texaco, Inc. of a 2.5 mile crude oil pipeline to the $10 \mathrm{G}$ lease automatic custody transfer of the Elk Hills Naval Peiroleum Reserve, Kern County, California.

- Opinion rendered in response to a request for consultation from the U.S. Department of Energy.

- Opinion concluded that "proposed construction, maintenance, and operation of the crude oil pipeline is not likely to jeopardize the continued existence of the San Joaquin kit fox, blunt-nosed leopard lizard, giant kangaroo rat or Tipton kangaroo rat. Critical habitat has not been determined for these species; therefore, none will be destroyed or adversely modified."

- Issued June 15, 1989; expires at end of project.

4. Formal endangered species consultation for the construction and operation by ARCO of a three-mile water pipeline on Naval Petroleum Reserve No. 2, Kern County, California.

- Opinion rendered in response to a raquest of consultation from the U.S. Department of Energy.

- Opinion concluded that "proposed construction, maintenance, and operation of the three-mile water pipeline on Naval Petroleum Reserve No. 2 is not likely to jeopardize the continued existence of the San Joaquin kit fox, blunt-nosed leopard lizard, giant kangaroo rat or Tipton kangaroo rat. Critical habitat has not been determined for these species; therefore, none will be destroyed or inodified."

- Issued August 17, 1989; expires at end of project.

5. Informal endangered species consultation concerning Phillip's Petroleum Company's proposed clean-up of site garbage or equipment not in use on Naval Petroleum Reserve No. 2. Kern County, California.

- Opinion rendered in response to a request for consultation from the U.S. Department of Energy.

- Opinion concluded that the proposed site clean-up is not likely to jeopardize the existence of the blunt-nosed leopard lizard, San Joaquin kit fox, or giant kangaroo rat.

- Issued December 7, 1989; expires at end of project. 
6. Formal endangered species consultation concerning coyote control in and adjacent to Naval Petroleum Reserves Number 1 and 2.

- Opinion rendered in response to a request for consultation from the U.S. Department of Agriculture Animal Damage Control.

- Opinion concluded that implementation of the subject program is likely to promote the conservation of the San Joaquin kit fox.

- Issued 2/28/89; expires at end of projict. 
IV. Kern County Health Department

Water and Waste Permits

Waste

Facility

Waste Type

Agency

Permit \#

$\underline{\text { Status }}$

365 Transfer

Solid

KCHD/CSWMB

SWIS 15-AA-

0269

Active

Station

(0270

Nonhazardous

Active

35R Transfer

Solid

0270

Inactive

Station

Nonhazardous

36R Landfill

()261

Solid

Inactive

Nonhazardous

35R Landfill

0260

Solid

Inactive

Nonhazardous

265 Iandfill

Solid

Inactive

Nonhazardous

25S Landfill

Solid

Inactive

Nonhazardous

KCHD/CSWMB

SWIS 15-AA-

0258

$\mathrm{KCHD} / \mathrm{CSWMB}$

SWIS 15-AA-

Inderground Storage Tanks

Facility

Agency

Permit \#

Status

35R\&36R Filling Stations

$\mathrm{KCHD}$

33088

Operating Permit

35R\&36R Filling Stations

$\mathrm{KCHD}$

A602-23

Removal Permit 
1. Deparment of Fish and Game Scientific Collector's Permit.

- Issued from the California Department of Fish and Game to:

David C. Anderson

Laura E. Briden

Kristie Hammer

Olga A. Jones

Thomas T. Kato

Wendy L. Philpott

Jerry H. Scrivner

Robert W. Stafford

R.on Stanfield

Steve Tabor

Greg D. Warrick

Bruce W. Zoellick

Kenı Ostler

Ted Doerr

Kurt Rautenstrauch

Cathy Wills

Ron Green
(Permit 5102)

(Permit 5110)

(Permit 5109)

(Permit 5108)

(Permit 5101)

(Permit 5106)

(Permit 5105)

(Permit 5103)

(Permit 5107)

(Permit 5100)

(Permit 5114)

(Permit 5113)

(Permit 5242)

(Permit 5239)

(Permit 5243)

- Authorized permitted to collect specimens according to condition listed in permit.

- Issued 3/24/89; expires 3/24/91.

2. Memorandurn of Understanding by and between EG\&G Energy Measurements, Inc., and California Department of Fish and Game.

- Authorized handling of the San Joaquin kit fox and the blunt-nosed leopard lizard.

- Issued 4/9/80; extended on several occasions; CDFG letter (dated 12/8/86) to EG\&G/EM authorized that the activities listed in the original MOU or its extnesion of August 17, 1983, could continue until the Department issues a new MOU. 
3. Memorandum of Understanding by and between EG\&G Energy Measurements, Inc., and California Depoment of Fish and Game.

- Authorizes EG\&G/EM to conduct field studies of special status small mammals; i.e., giant kangaroo rate, Tipton kangaroo rat, short-nosed kangeroo rat, Salina pocket mouse, San Joaquin pocket mouse, Buena Vista Lake shrew, and San Joaquin antelope squirred.

- Issued 8/22/88; expires 10/31/90.

4. Memorandum of Undertanding by and between EG\&G Energy Measurement, Inc., and California Departmnet of Fish and Game.

- Authorized EG\&G/EM to capture and translocate foxies to Naval Petrleum Reserve No. 1.

- Issued 7/8/88; expires 12/31/91. 


\title{
APPENDIX M
}

\author{
AUDIT OF ENVIRONMENTAL SERVICES \\ DEPARTMENT HAZARDOUS WASTE DISPOSAL SITE
}




\title{
Bechtel Petroleum Operations, Inc.
}

\author{
Interoffice Memorandum
}

\section{To File \\ subject AUDIT OF ESD HAZARDOUS \\ WASTE DISPOSAL SITE}
cooten to C. E. Emerick
R. L. Donahoe
G. N. Gough

\author{
Fllo No $7.2,9.2$ \\ Dat. September 18,1989 \\ From R. E. Campbell \\ a Envixonmental services \\ A NPRC/21G En 6620
}

On July 27, 1989, I performed an audit of the Envirosafe services of Idaho hazardous waste disposal facility at Grandview, Idaho. The facility is essentially unchanged from the previous audit conducted by Curt Morgan, in March of 1988. The single exception is that the treatment/stabilization processing plant is in full operation. Other minor changes are noted in the attached evaluation form.

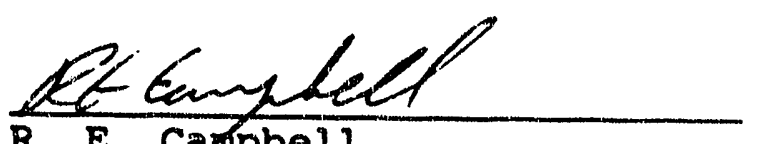

RLD: rbe 
Company: Environsafe Services of Idaho/Division of IU Inc.

Site Location: Grand View, Idaho

Date of visit: $7 / 27 / 89$

Person performing site inspection:

I. Geology and Hydrology

A. Climate - Rainfall and Evaporation

9 " rainfall

80 " evaporation

B. Distance to Groundwater

- 190-200 ft. perched water non-potable

- 120 potential monitoring wells

*- Currently monitoring 36 wells

* $3000+$ to potable water table

c. Depth and Consistency of Formation

* - 50' of sand gravel

* - 70' black clay/brown clay e $50^{\prime}$

* $130^{\circ}$ gray clay

* $20^{\prime}$ shale

*-40' shale

D. Potential Earthquake Damage and Final Location of Waste Not in seismic zone.

II. Demographics

A. Nearby Population Centers

- Grandview, Idaho

- Mnt. Howe, Idaho

B. Routes to the site

I-84 \& state routes

C. Potential Danger to Nearby Communities

Minimurn

* new information or supplement $7 / 27 / 89$ 
III. Description of Personnel safety Program

Well organized.

IV. Site Safety Program

A. Precautions Against on Site Safety Accidents

Good Training

B. Safe Handling of Material

Good Training

V. Management of on site wastes

A. Segregation of Non Compatible Material

All segregated

B. Processing and treatment of wastes

* - Stabilization plant in operation uses mechanical mixing in $110 \mathrm{~cm}$. yd. bin

C. General Housekeeping

Good

D. On site Road Conditions

Good

E. Level of Sophistication and Professionalism

High

VI. In site Equipment

A. Maintenance and cleanliness

- A lot of equipment. only run one shift.

- No trucking/but can get a sub if we need it.

- All new cells double lines with 60 \& $80 \mathrm{mil}$.

B. Operators Protected Adequately

Yes

* new information or supplement $7 / 27 / 89$ 
VII. Laboratory

A. Sampling Techniques and Chain of Custody

*- Can get profile approved in 1 week - stabilization Ionger - 3 weeks

*- Composite every 10 drum. EPA may change. Every drum.

*- Open every drum for inspection - lab tech.

B. Adequate Record Keeping

- Participate 2 times yearly in EPA performance evaluation study.

- still in effect - $7 / 89$

c. Personnel

*Excellent. 4 chemists, 3 technicians

D. Equipment

- GC's

- AA's

VIII.Reliability (Days fully operations in past 5 years) Open, but slowed down in 1983 due to 10,000+ drum inventory.

IX. Permit status

A. Copies of Permits

- Part A Interim Status

- Part B Draft Pexmit out from EPA this summer

* Complete sampling under review.

B. What Wastes Cannot be Accepted

- Radioactive

- Biological

- Reactive Wastes

- No Halogenated HC above $1000 \mathrm{pm}$

- No Dioxins

- Explosives

$X$. Insurance coverage and any Long Term Indemnification offered $\$ 3 M / 6 M$ coverage with Alexander \& Alexander

XI. Audited Financial statement.

Will mail.

* new information or supplement $7 / 27 / 89$ 
XII. Does TSDF Offer a Written Contract:

Yes.

XIII.Assurance of Proper Disposal

- Can get approval for shipment in 1 week. Must schedule ahead of time.

- Every drum inspected 1.1x usually $2 x$

XIV. Disposal Rates for Various Materials

- Larry Brewer

XV. Names and Numbers of Local, state and Federal Agencies that will verify the above.

* - scott Lund

Health \& Welfare

Department of Environment

State of Idaho

(208) $334-5887$

- Bill Freutel

EPA Region $X$

(208) 344-1450

* new information or supplement $7 / 27 / 89$ 
Company: Environsafe Services of Idaho/Division of IU Inc.

Site Location: Grand Vlew, Idaho

Date of visit: $7 / 27 / 89$

Person performing site inspection:

I. Geology and Hydrology

A. Climate - Rainfall and Evaporation

9 " rainfall

80" evaporation

B. Distance to Groundwater

- 190-200 ft. perched water non-potable

- 120 potential monitoring wells

* - Currently monitoring 36 wells

* $3000+$ to potable water table

c. Depth and consistency of Formation

* $50^{\prime}$ of sand gravel

* $70^{\prime}$ black clay/brown clay a $50^{\prime}$

* $130^{\prime}$ gray clay

* - $20^{\prime}$ shale

* 40' shaie

D. Potential Earthquake Damage and Final Location of Waste Not in seismic zone.

II. Demographics
A. Nearby Population Centers
- Grandview, Idaho
- Mnt. Howe, Idaho
B. Routes to the site
I-84\& state routes
C. Potential Danger to Nearby Communities
Minimum
* new information or supplement $7 / 27 / 89$ 
III. Description of Personnel Safety Program

Well organized.

IV. Site Safety Program

A. Precautions Against on site Safety Accidents

Good Training

B. Safe Handling of Material

Good Training

V. Management of on site wastes

A. Segregation of Non Compatible Material

All segregated

B. Processing and treatment of Wastes

* - stabilization plant in operation uses nechanical mixing in $110 \mathrm{~cm}$. $y d$. bin

C. General Housekeeping

Good

D. On Site Road Conditions

Good

E. Level of Sophistication and Professionalism

High

VI. In Site Equipment

A. Maintenance and Cleanliness

- A lot of equipment. Only run one shift.

- No trucking/but can get a sub if we need it.

- All new cells double lines with 60 \& $80 \mathrm{mil}$.

B. Operators Protected Adequately

Yes

* new information or supplement $7 / 27 / 89$ 
VII. Laboratory

A. Sampling Techniques and chain of Custody

* - Can get profile approved in 1 week - stabilization longer - 3 weeks

* - Composite every 10 drum. EPA may change. Every drum.

* - Oper every drum for inspection - lab tech.

B. Adequate Record Keeping

- Participate 2 times yearly in EPA performance evaluation study.

- otijl in effect - $7 / 89$

c. Personnel

*Excelient. 4 chemis:s, 3 technicians

D. Equipment

- GC:s

- AA's

VIII.Reliability (Days fully operations in past 5 years)

Open, but slowed down in 1983 due to $10,000+$ drum inventory.

IX. Permit status

A. Copies of Permits

- Part A Interim Status

- Part B Draft Permit out from EPA this summer

* - Complete sampling under review.

B. What Wastes cannot be Accepted

- Radioactive

- Biological

- Reactive Wastes

- No Halogenated HC above 1000 pin

- No Dioxins

- Explosives

$x$. Insurance covinrage and any Long Term Indeninification offered $\$ 3 M / 6 \mathrm{M}$ coved age with Alexander \& Alexander

XI. Audited Financial statement.

Will mail.

* new information or supplement $7 / 27 / 89$ 
XII. Does TSDF offer a Written contract:

Yes,

XIII.Assurance of Proper Disposal

- Can get approval for shipment in l week. Must schedule ahead of time.

- Every drum inspected $1.1 x$ usually $2 x$

XIV. Disposal Rates for Varjous Materials

- Larry Brewer

XV. Names and NUmbers of Local, State and Federal Agencies that will verify the pove.

* - Scott Iund

Health Welfare

Department of Environment

State of Idaho

(208) 334-58C ।

- Bill Freutel

EPA Region $X$

(208) $344-1450$

* new information or supplement $7 / 27 / 89$ 
Company: Environsafe Services of Idaho/Division oE IU Inc.

Site Location: Grand View, Idaho

Date of visit: $7 / 27 / 89$

Person performing site inspection:

I. Geology and Hydrology

A. Climate - Rainfall and Evaporation

9 " rainfall

80" evaporation

B. Distance to Groundwatex

- 190-200 ft. perched water non-potable

- 120 potential monitoring wells

* - Currently monitoring 36 wells

* $3000+$ to potable water table

C. Depth and Consistency of Formation

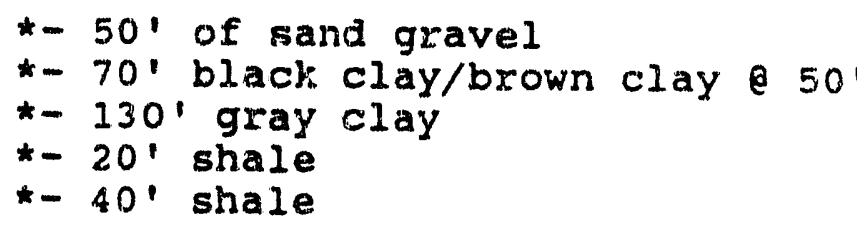

D. Potential Earthquake Damage and Final Location of Waste Not in seismic zone.

II. Demographics

A. Nearby Population Centers

- Grandview, Idaho

- Mnt. Howe, Idaho

B. Routes to the site

I-84\& State routes

c. Potential Danger to Nearby Communities

Minimum

* new information or supplement $7 / 27 / 89$ 
III. Description of Personnel safety Program

Well organized.

IV. Site safety Program

A. Precautions Against on Site safety Accidents

rood Training

B. Safe Handing of Material

Good Training

v. Management of on site Wastes

A. Segregation of Non Compatible Material

All segregated

B. Processing and treatment of wastes

* - stabilization plant in operation uses mechanical mixing in $110 \mathrm{~cm}$. $y d$. bin

C. General Housekeeping

Good

D. On site Road conditions

Good

E. Level of Sophistication and Professionalism

High

VI. In Site Equipment

A. Maintenance and Cleanliness

- A lot of equipment. Only xun one shift.

- No trucking/but can get a sub if we need it.

- All new cells double lines with 60 \& $80 \mathrm{mil}$.

B. Operators Protected Adequately

Yes

* new information or supplement $7 / 27 / 89$ 
VII. Laboratory

A. Sampling Technigues and chain of custody

*- Can get Profile approved in 1 week - stabilization longer - 3 weeks

*- Composite every 10 drum. EPA may change. Every drum.

*- Open every drum for inspection - lab tech.

B. Adequate Record Keeping

- Participate 2 times yearly in EPA performance evaluation study.

- Still in effect - 7/89

c. Personnel

*Excellent. 4 chemists, 3 techricians

D. Equipment.

- GC's

- AA's

VIII.Reliability (Days fully operations in past 5 years)

Open, but slowed down in 1983 due to 10,000+ drum inventory.

IX. Permit status

A. Copies of Permits

- Part A Interim Status

- Part B Draft Permit out from EPA this summer

* - Complete sampling under review.

B. What Wastes Cannot be Accepted

- Radioactive

- Biological

- Reactive Wastes

- No Halogenated HC above $1000 \mathrm{pm}$

- No Dioxins

- Explosives

$x$. Insurance coverage and any Long Term Indemnification cffered $\$ 3 M / 6 M$ coverage with Alexander \& Alexander

$X I$. Audited Financial statement

will maj.l.

* new information or supplement $7 / 27 / 89$ 
XII. Joes TSDF offer a written Contract:

Yes.

XIII. Assurance of Proper Disposal

- Can get approval for shipment in 1 week. Must schedule ahead of time.

- Every drum inspected $1.1 x$ usuálly $2 x$

XIV. Disposal Rates for Various Materials

- Larry Brewer

XV. Names and NUmbers of Local, state and Federal Agencies that will verify the above.

* - Scott Lund

Health \& Welfare

Department of Environment

State of Idaho

(208) 334-5887

- Bill Freutel

EPA Region $X$

(208) 344-1450

* new information or supplement $7 / 27 / 89$ 

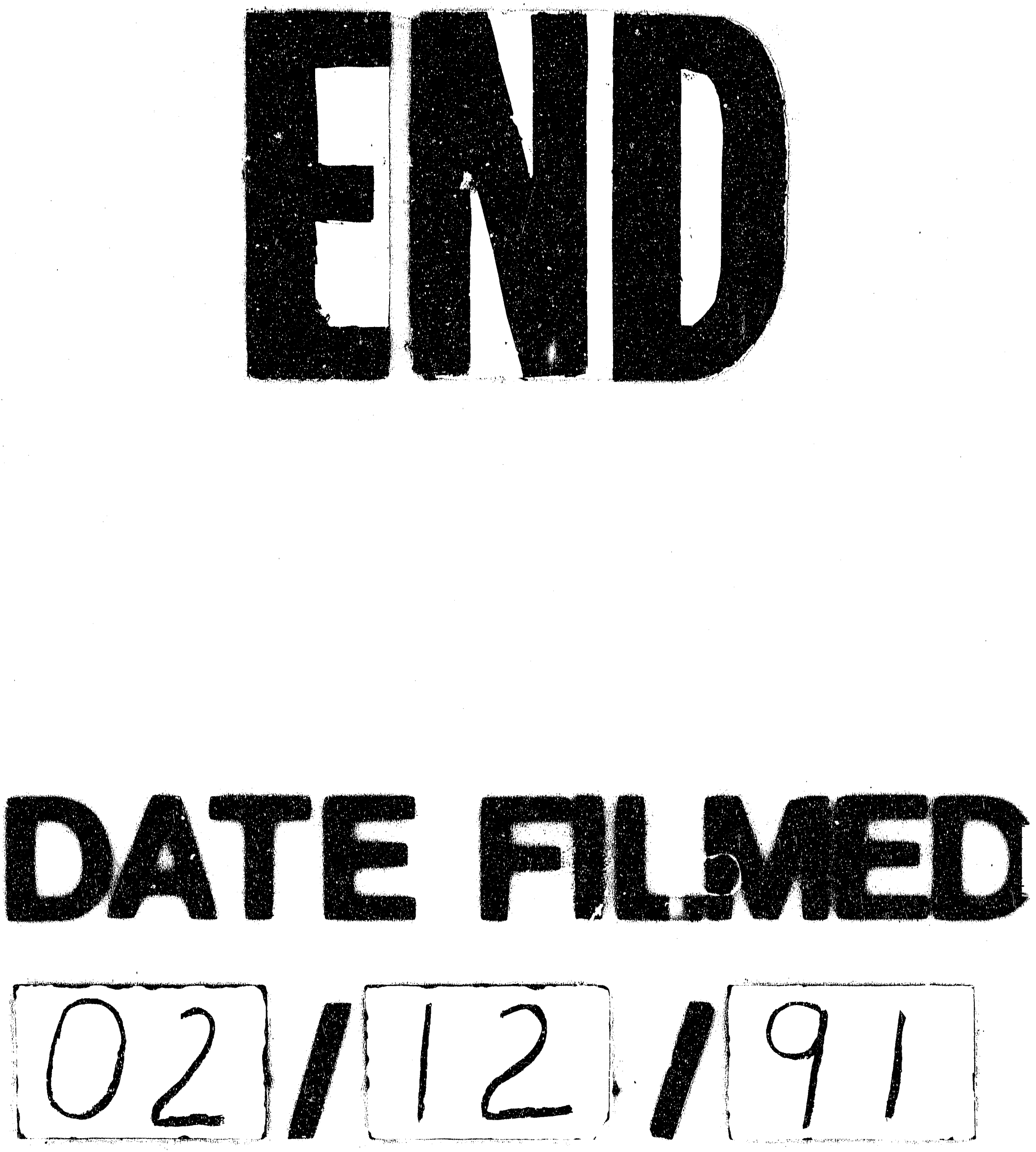
